This document is confidential and is proprietary to the American Chemical Society and its authors. Do not copy or disclose without written permission. If you have received this item in error, notify the sender and delete all copies.

\title{
Engineering Functional Polymer Capsules Towards Smart Nanoreactors
}

\begin{tabular}{|r|l|}
\hline Journal: & Chemical Reviews \\
\hline Manuscript ID: & cr-2015-002417.R2 \\
\hline Manuscript Type: & Thematic Review \\
\hline Date Submitted by the Author: & 20 -Aug-2015 \\
\hline Complete List of Authors: & $\begin{array}{l}\text { Voit, Brigitte; Leibniz Institute of Polymer Research Dresden, } \\
\text { Gaitzsch, Jens; University College London, Chemistry } \\
\text { Huang, Xin; Harbin Institute of Technology, }\end{array}$ \\
\hline
\end{tabular}

SCHOLARONE ${ }^{\text {'w }}$

Manuscripts 


\section{For Chemical Reviews}

Special thematic issue on "Frontiers in Macromolecular and Supramolecular

Science"

\section{Engineering Functional Polymer Capsules Towards Smart Nanoreactors}

Jens Gaitzsch ${ }^{a), b)^{*}}$, Xin Huang ${ }^{c}{ }^{*}$, Brigitte Voit $^{()^{*}}$

a) Department of Chemistry, University College London, London WC1H OAJ, United Kingdom

b) Department of Chemistry, University of Basel, Klingelbergstrasse 80, 4056

Basel, Switzerland

c) School of Chemical Engineering and Technology, Harbin Institute of Technology, 150001, Harbin, China

d) Leibniz-Institut fuer Polymerforschung Dresden e.V., Hohe Strasse 6, 01069

Dresden, Germany

Email addresses: jens.gaitzsch@unibas.ch; xinhuang@hit.edu.cn; voit@ipfdd.de 


\section{Contents}

1. Introduction

2. Synthetic Functional Polymer Capsules

2.1 Formation of Polymersomes

2.1.1 Formation from Bulk Material

2.1.2 Formation from Solution

2.1.3 Formation during Polymerisation

2.2 Bulk Polymeric Capsules

2.2.1 Template-Based Polymerisation

2.2.2 Miniemulsion Polymerisation

2.3 Proteinosomes

3. Engineering Functional Polymer Capsules Towards Smart Nanoreactors

3.1 Membrane-Based Functionalisation of Polymer Capsules

3.1.1 Channel Proteins as Transporters

3.1.2 pH Responsive Polymersomes

3.1.3 Temperature Responsive Vesicles

3.1.4 Light Responsive Polymersomes

3.1.5 Reduction and Oxidation Sensitive Polymersomes

3.1.6 Solvent Responsive Vesicles

2

ACS Paragon Plus Environment 


\subsubsection{Combined Responsiveness}

3.2 Core-Based Design

3.2.1 Multi-Compartment Model

3.2.2 "Yolk-Shell" Model

4. Application of the Polymer Capsule Nanoreactors

4.1 Application of the Polymer Capsule Nanoreactors for Enzyme Catalysis

4.2 Application the Polymer Capsule Nanoreactors for Polymerisation

4.3 Application of the Polymer Capsule Nanoreactors for Nanoparticles Synthesis

4.4 Artificial Organelles

5. Conclusion 


\section{Introduction}

The design and construction of integrated chemical ensembles for studying complex biological systems is attracting considerable interest in a wide range of research communities. ${ }^{1}$ Amongst these studies, one of the most successful stories is the construction of hollow compartments, i.e. in the form of nano-or microscale capsules with an aqueous core surrounded by an hydrophobic domain. $^{2}$ Such a constructed model is of considerable interest due to promising applications in materials science, biomedicine, and catalysis. ${ }^{3-4}$ The domain of the hollow compartments can either be of inorganic nature or can be made up of organic molecules such as lipids (e.g. liposomes) and polymers. ${ }^{5}$ To date, a great variety of polymer-based compartments has been demonstrated, including polymeric capsules $^{6-8}$ and vesicles of an ordered membrane of amphiphilic block-copolymers. ${ }^{9-11}$ In comparison with other reported hollow compartments, ${ }^{5,12}$ the main difference lying in polymeric vesicles is their polymer membrane which endows the structure with stability, flexibility, and functionality. It should be noted that a specific block length ratio in the block-copolymers is essential for the formation of vesicles. For a fixed hydrophilic part, with an increasing hydrophobic section of the amphiphilic block-copolymer, first micelles are formed, then worms, vesicles and finally tubular vesicles. ${ }^{13}$ Depending on the origin of the block-copolymer, the vesicles are called polymersomes ${ }^{2,14}$ (for non-branched synthetic polymers), 
dendrimersomes ${ }^{15}$ (for dendrimers) or recently reported proteinosome $\mathrm{e}^{16-17}$ (for synthetic proteins) either as mono- or multi-compartment capsules. ${ }^{18-19}$ Most commonly, polymersome is also used as the governing term for the named structures. In comparison to their lipid counterparts, polymersomes exhibit a low critical aggregate concentration (CAC), meaning that there is only an insignificant number of individual polymer chains in the solution after reaching $\mathrm{CAC}^{20}$ This means that polymersomes are non-ergodic, have no exchange of block-copolymer chains between separate vesicles and show a greatly enhanced mechanical stability over liposomes. ${ }^{11,21}$

With respect to their functionality, the recent development of polymer chemistry allows the tailor-made adjustment of membrane properties, including that they can be functionalized at the outer surface for targeting approaches and possess stimuli-responsiveness for release of encapsulated compounds "on demand". 22-26 Besides their application as drug-delivery-systems, these properties make polymeric vesicles ideal candidates to be nanoreactors by encapsulating active compounds (enzymes, proteins, enzyme mimics) with the dual role of protecting the compounds from proteolytic attack and serving as a reaction space..$^{10,27-30}$ The necessary exchange of substrates/products with the environment to support in situ reactions occurs through porous, synthetic membranes or by the insertion of channel proteins depending on the chemical nature of the copolymer. ${ }^{31-33} \mathrm{~A}$ significant step beyond this is also realized by the exploitation of the designed 
nanoreactors to be artificial organelles. ${ }^{34-36}$ Such synthetically constructed models represent a new emerging field, since on the one hand they show promising potential for therapeutic applications in order to actively produce drugs to fight disease (enzyme replacement therapy) and to enhance the activity of existing organelles in cases of cellular stress; and on the other hand they could be exploited to be an artificial cell to contribute to the view of origin of life.

In this regard, a number of reviews have been published covering a number of structures from polymersomes, ${ }^{2,37}$ biohybrid polymer capsules ${ }^{38-40}$ to multi-compartmentalized polymeric system ${ }^{18-19}$ with the application in the field of drug delivery, biomedicine or biomimicry etc. In this review, we specially focus our interest on the recently published papers about the engineering of a wide range of polymer-based capsules towards smart nanoreactors and artificial organelles. We start with a discussion on the preparation of polymer vesicles based on different techniques, including self-assembly, template-based polymerisation, in situ polymerisation, emulsion polymerisation, microfluidics or Pickering emulsion etc. (Section 2). Layer-by-layer method is not included, since it has been widely summarized very recently by Caruso et al.. ${ }^{41-44}$ Then the engineering of polymer capsules to be nanoreactors from the membrane domain-based design to core domain-based design is discussed in Section 3. Subsequently, we highlight the potential application of the constructed polymer capsule-based nanoreactors 
in controlled enzyme catalysis, polymerisation, nanoparticles synthesis and artificial organelles in Section 4.

\section{Synthetic Functional Polymer Capsules}

\subsection{Formation of Polymersomes}

Polymersomes are, as stated in the introduction, hollow structures with an ordered polymer membrane. These can be formed of an amphiphilic $A B$ block-copolymers to yield a polymer bilayer or of $A B A / A B C$ triblock-copolymers forming a mono/multilayer. Both versions have the hydrophobic block (A or $\mathrm{C}$ ) at the outer surface and in the centre of the membrane. The first challenge when working with polymersomes is thus finding a method that ensures a controlled membrane formation with the amphiphilic block-copolymer of interest. As polymeric structures (micelles and vesicles) are generally very stable once formed, it is even more important to target the correct self-assembly structure directly. Due to their stability polymersomes are unlikely to rearrange themselves into an energetically more stable one due to a high kinetic barrier. This barrier prevents single polymer chains to go into solution and interchange with other structures formed. Such a behaviour results in a low critical aggregation concentration (CAC) and a low rate of molecules interchange which is described as ergodicity. Polymeric aggregates are consequently non-ergodic, which distinguishes them from lipid vesicles and micelles, which show a considerable amount of ergodicity. It 
should be noted that this lack of ergodicity can also be shown in the lack of mobility in polymeric membranes as compared to lipid ones. Given the correct combination of polymer and self-assembly method, it is then possible to trigger the formation of custom-made assemblies, even of mixtures from the classical micelles, worms, vesicles and tubes to give round-bottom flask-like assemblies, for example. ${ }^{45}$

There are generally three ways to form polymersomes. One way is to start from a thin polymer film. In here the bulk material is brought into contact with water and then swells selectively to form the final structures. This is a "top down" approach and it is commonly referred to as thin film rehydration. ${ }^{20}$ The opposite of this approach is then logically the "bottom up" approach starting from a completely dissolved amphiphilic block-copolymer and then triggering the self-assembly process. $^{20}$ The third way has only been reported very recently by Armes et al. ${ }^{46}$ and describes polymersomes formation during polymerisation. The authors named it a polymerisation-induced self-assembly or PISA process. All of these approaches will now be covered in detail in the following sections.

\subsubsection{Formation from Bulk Material}

In this approach, the polymersomes are formed from a previously created polymeric thin film which is then rehydrated with a suitable solvent, which is why this method is also referred to as film-rehydration. To create the initial film, 
the polymer, an amphiphilic block-copolymer, is dissolved in a good solvent for both blocks in a concentration of $5-10 \mathrm{mg} / \mathrm{ml}$. When the solvent is then removed, the polymer creates a thin film on the vessel wall (Figure 1)..$^{20,47-49}$ This dry film is now wetted with a good solvent for exclusively one of the blocks. In the case of water based systems, this is done with either plain water or buffer solution such as phosphate buffered saline (PBS), while organic solvent systems are also known. ${ }^{50-52}$ Water now diffuses into the film, causing the hydrophilic part to swell. It is very important to ensure that the polymer stays as a film during the hydration and that no small patches of bulk material get into the solution as the necessary shear force from the stirring must be present at all times. ${ }^{21,49}$ A feasible method to prevent this is to avoid the use of low molecular-weight polymers (below $10 \mathrm{~kg} / \mathrm{mol}$ classical film rehydration is not reported) as they are less entangled, leading them to detaching from one another easier than longer polymers. ${ }^{53-54}$

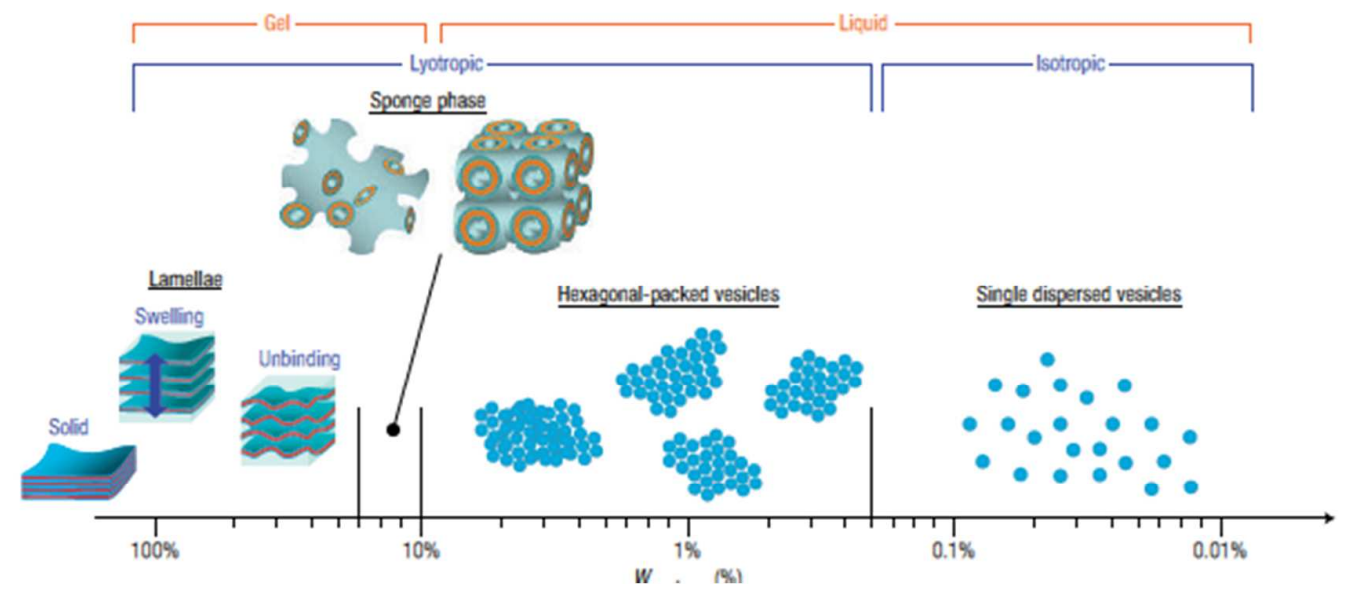

Figure 1. The evolution of a polymersome from a thin film to a complete vesicle shown in dependence of the water/polymer ratio and molecular weight. After the first lamellae 
phases are formed, packed vesicles (sponge phase) are formed initially and later on free ones appear in solution. ${ }^{49}$ (Reproduced with permission from reference 49. Copyright (2005) Nature Publishing Group).

Eventually, pre-vesicle structures such as tubes and genius-like particles detach from the edge of the film into the solution. ${ }^{21,49,55-57}$ When they now develop further the hydrophobic part of the polymer needs to be shielded off by the hydrophilic block. ${ }^{9,54,58}$ The polymer now slowly arranges into a structure where this shielding is achieved best, depending on the block length ratio (see introduction). It can only happen slowly since the arrangement of the polymer chains happens through the reportedly slow diffusion of them. Literature knows this process to take up to 8 weeks, but may be speeded up by the application of ultrasound. ${ }^{59-61}$

Film rehydration is thus a long process and not suitable for short-term evaluation whether a polymer is feasible to form vesicles, or for polymers that show hindered diffusion due to high glass transition or crystallinity like polystyrene (PS) or poly(L-lactic acid) (PLLA). ${ }^{20-21,49}$ Also, hydrolysable polymers are not feasible as the may degrade during the long formation process. Besides this general approach to film rehydration, some derived methods have been developed further by Battaglia et al., to make it also feasible for shorter polymer chains. Battaglia et al. showed that if the polymer chain is short enough (e.g. below $5 \mathrm{~kg} / \mathrm{mol}$ ), no stirring is necessary and the vesicle evolution process occurs just by putting the polymer in contact with 
water. If mixed with a fluorescent dye, the process can now actually be monitored by fluorescence microscopy. ${ }^{49}$ The same group now went a step further and developed a film rehydration from a patterned surface. ${ }^{62}$ When altering the surface hydrophobicity in a 1000 or 2000 mesh grid (1.0 and 2.0 $\mathrm{mm}$ grid spacing), the size of polymersomes created is homogeneous and within micrometre range. This is in contrast to classic film rehydration which leads to a broad size range of vesicles. ${ }^{62}$

Electroformation is another form of film rehydration to create polymersomes. It has been developed originally for liposome formation where the building blocks (lipids) have a significantly smaller molecular weight. ${ }^{63-64}$ In this method the film is put on a conductive surface (indium tin oxide (ITO) on glass) in an alternating current at varying frequencies and voltages. Both parameters have to be adapted for the polymer system used. However, while is has been shown that polymersomes can be formed that way, ${ }^{65}$ It has proven difficult to detach the polymers from the substrate, yielding dome-like structures in many cases, probably due to their higher molecular weight compared to lipids(Figure 2) ${ }^{66}$ 

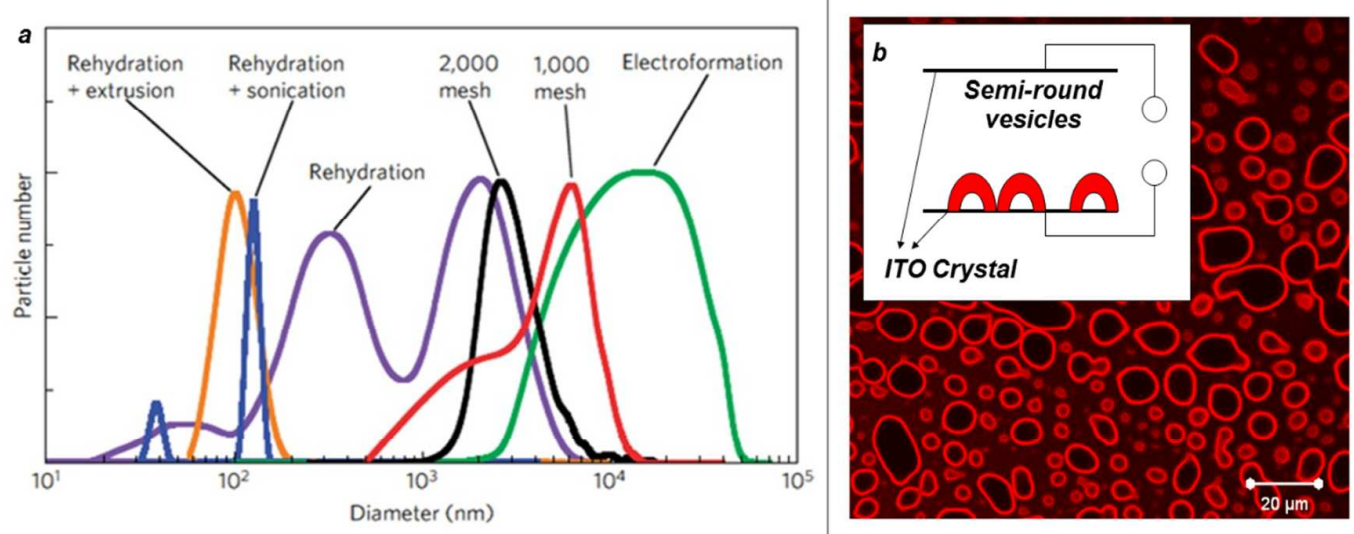

Figure 2. Vesicle sizes achieved by different kinds of film rehydration, controlled and uncontrolled and also using electroformation. ${ }^{62}$ Electroformation itself is shown on the left where dome-like structures evolve with PEG-PDEA. ${ }^{66}$ (Part (a) reproduced with permission from reference 62. Copyright (2009) Nature Publishing Group.)

In summary, thin film rehydration can yield vesicles of a broad variety in size, from $100 \mathrm{~nm}$ until 100 micrometers, depending on the specific conditions used and the polymer applied.

\subsubsection{Formation from Solution}

Besides the "top down" approach just described, the "bottom up" way to form polymersomes from dissolved unimeric polymer chains is also widely used. Depending on the amphiphilic block-copolymer structure, this switch can be accomplished by either exchanging an organic solvent to water ${ }^{67}$ or by changing the properties of the solvent $(\mathrm{pH} \text {, temperature })^{13,56}$ which yields in the one block of the polymer becoming insoluble. The assembly transitions through micelles, octopus-like assemblies to worms and vesicles via different 
pathways which occur simultaneously (Figure 3).

The principle of these formations is always the same and was one of the first methods used for polymersome preparation by Discher and Eisenberg. ${ }^{2,14}$ First of all, the whole polymer is dissolved in an organic solvent suitable for both blocks. Once the polymer is dissolved into single polymer chains, e.g. unimers, the polarity is slowly raised by the means of adding water. At a certain point (which depends on the polymer and solvent used), the water content of the solution becomes too large to dissolve the hydrophobic part of the polymer. From this point onwards polymer-polymer interactions are favoured over polymer-solvent interactions and the self-assembly process is induced. One can see from this approach that not all organic solvents are suitable for this approach, but that it has to be miscible with water. ${ }^{14,20}$ Hence, only polymers that are soluble in water-miscible organic solvents can be used applying this organic solvent-to-water solvent switch. 


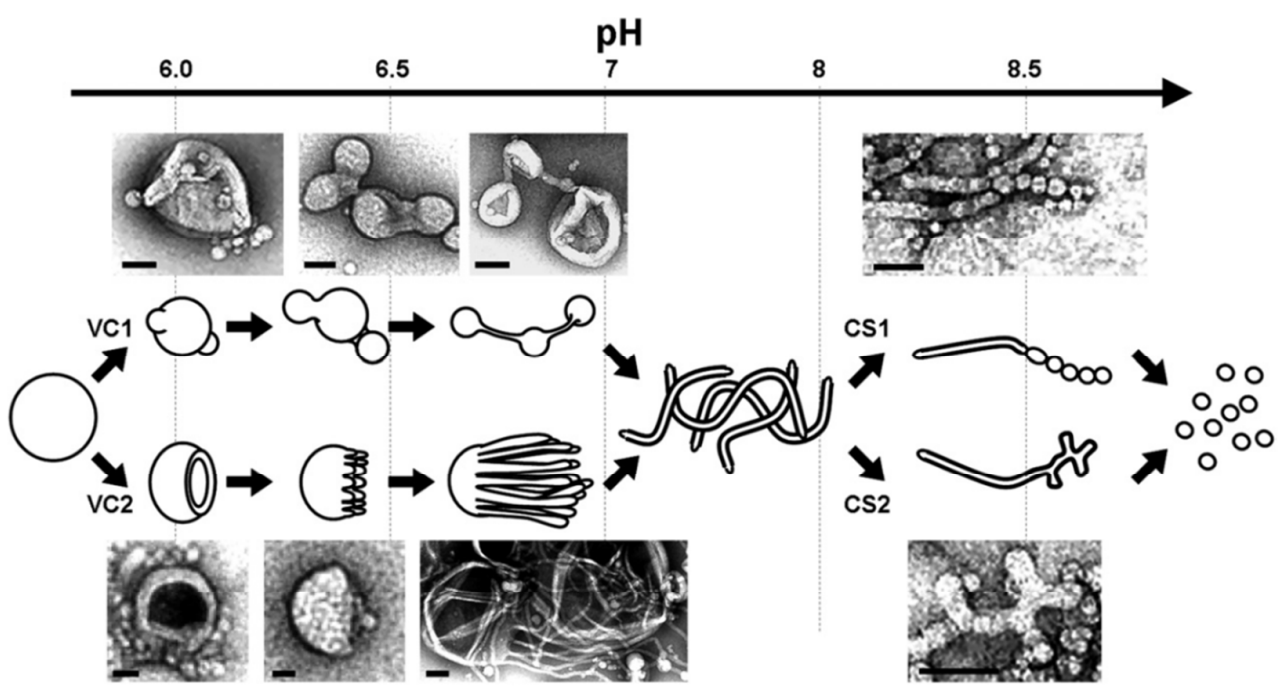

Figure 3. Possible structures observed when using a $\mathrm{pH}$ guided assembly of poly((butyl)methacrylate)-poly(methacrylic acid) PBMA-PMA via 2 pathways of vesicle creation (VC1 and VC2) and the final creation of spheres (CS1 and CS2). ${ }^{68}$ (Reproduced with permission from reference 68. Copyright (2009) Royal Society of Chemistry).

Once the polymer begins to self-assemble, it is necessary to make a very slow transition process in order to yield the thermodynamically stable polymersomes and not kinetically favoured micelles. ${ }^{2,69}$ It is the stability of polymer aggregates that hinders their transition into the thermodynamically more stable polymersomes. This is mainly due to the amount of energy needed to dissolve a single chain from the aggregates, also reflected by the very low critical aggregation concentration (CAC) of polymers in comparison to lipids. ${ }^{64,70-71}$ A slow solvent transition will thus favour the thermodynamically stable state of the vesicle.

Apart from switching the polarity of the solvent, one can also change the $\mathrm{pH}$ or temperature of the solvent to induce self-assembly, if suited polymers are 
used in the hydrophobic part. Here, the whole polymer is dissolved in water in a state where it is completely soluble, using sensitive polymers. Temperature sensitive polymers like PNIPAM with an LCST for example, are soluble at low temperatures. With rising temperatures, the hydrogen bonds keeping the polymer in solution break and thus induce the self-assembly process into polymersomes. ${ }^{72-73}$ Temperature-sensitive peptidosomes can, of course, be formed in the same way. ${ }^{74}$ Kiick et al. report a diethylene glycol functionalised polymethacrylate, PDEGMEMA, which shows a temperature-dependent solubility switch when conjugated with a collagen-like polymer. ${ }^{75}$ In case of $\mathrm{pH}$-sensitive polymers it is logically the $\mathrm{pH}$ switch which induces the self-assembly process. ${ }^{59,66,76}$ Again, the changes in $\mathrm{pH}$ or temperature need to be done slowly due to the non-ergodic behaviour of polymers as stated above. Also for polyionic polymers it has been shown that immediate complexation by counterions does induce vesicle formation. After complexation the charges get masked and the previously water-soluble polyion becomes hydrophobic, inducing the self-assembly into vesicles. ${ }^{77}$ Essentially, not even charges are necessary, but the polymer must be able to complex the ions added. Lee et al. showed this by the complexation of $\mathrm{Hg}^{2+}$ ions by polyimidazoles. The binding forces of the ligand-metal complex were enough to induce vesicle and micelle formation. The morphology of the aggregates form is governed the amount of mercury added (Figure 4). ${ }^{78}$ Independent of the actual external trigger used, the polymersomes produced in that approach are always around $100-200 \mathrm{~nm}$ 
in size.

The complexing agent does not necessarily have to be a salt, but can also be an oppositely charged polyion. These complexes do form vesicles and are known as polymer-ionic-complex-vesicles (PICsomes). ${ }^{79-80}$ In contrast to the examples just discussed, the second polyion does not need to be added slowly, which is a notable exception from other vesicle formation methods. PICsomes will form if the hydrophilic-to-hydrophobic balance is correct. Once formed, PICsomes have similar properties as classic polymersomes. ${ }^{27,79,81-82}$

Below CMC

2: $\mathrm{Hg}^{2+}=1: 1$ binding

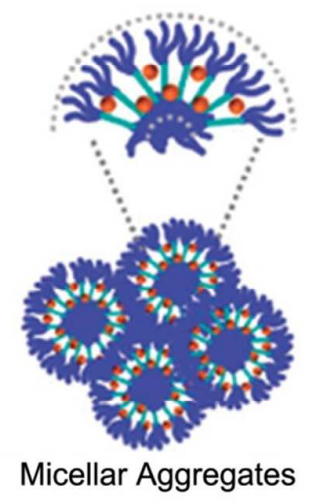

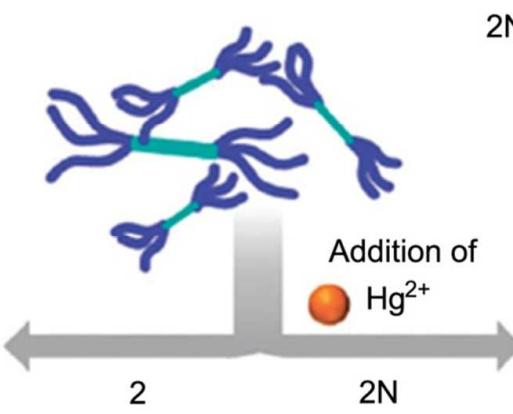

2
$2 \mathrm{~N}: \mathrm{Hg}^{2+}=1: 2$ binding

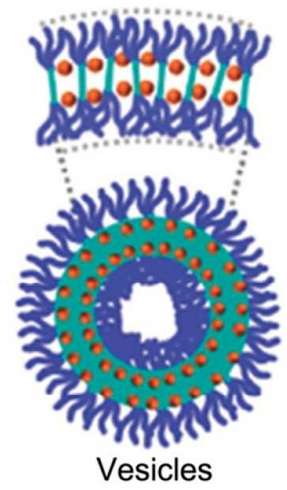

Figure 4. Schematic representation of the $\mathrm{Hg}^{2+}$-induced self-assembly of polyions towards micelles and vesicles through complexation. ${ }^{78}$ (Reproduced with permission from reference 78. Copyright (2014) Royal Society of Chemistry).

There is also an extrusion-based way of performing a stepwise solvent switch which was developed by Lecommandoux et al. (Figure 5). Here, water is added to a solution of the polymer in an organic solvent which is not miscible with water (Toluene). A similar process for liposomes was the role-model for 
polymersomes made this way. ${ }^{83}$ The polymer now aggregates at the water-organic solvent interface, stabilising the water droplet and thus forming a water-in-oil emulsion. ${ }^{18,84}$ Hence, this method is also referred to as forming polymersomes by emulsion. The size of the droplets in the emulsion determines the size of the final polymersomes formed, making large or giant polymersomes of several micrometers possible. The water-oil-emulsion is now extruded into an aqueous solution with higher density than the organic solvent. Adjusting the density is achieved by using a sucrose solution. Once the water droplets of the emulsion reach the sucrose-solution/oil interface, the polymer bilayer originally stabilising this interface assembles around the polymer-stabilised water droplet. As a result, the water droplet has a second polymer layer around it and is in an aqueous environment, hence it is now a polymersome. ${ }^{84-85}$ The water used to create the emulsion can already contain small polymersomes produced by a different process which could in this way now be encapsulated in a bigger polymersome. Lecommandoux et al., who pioneered the emulsion/extrusion technique, have shown the production of such polymersomes-in-polymersomes aggregates. ${ }^{86}$ 


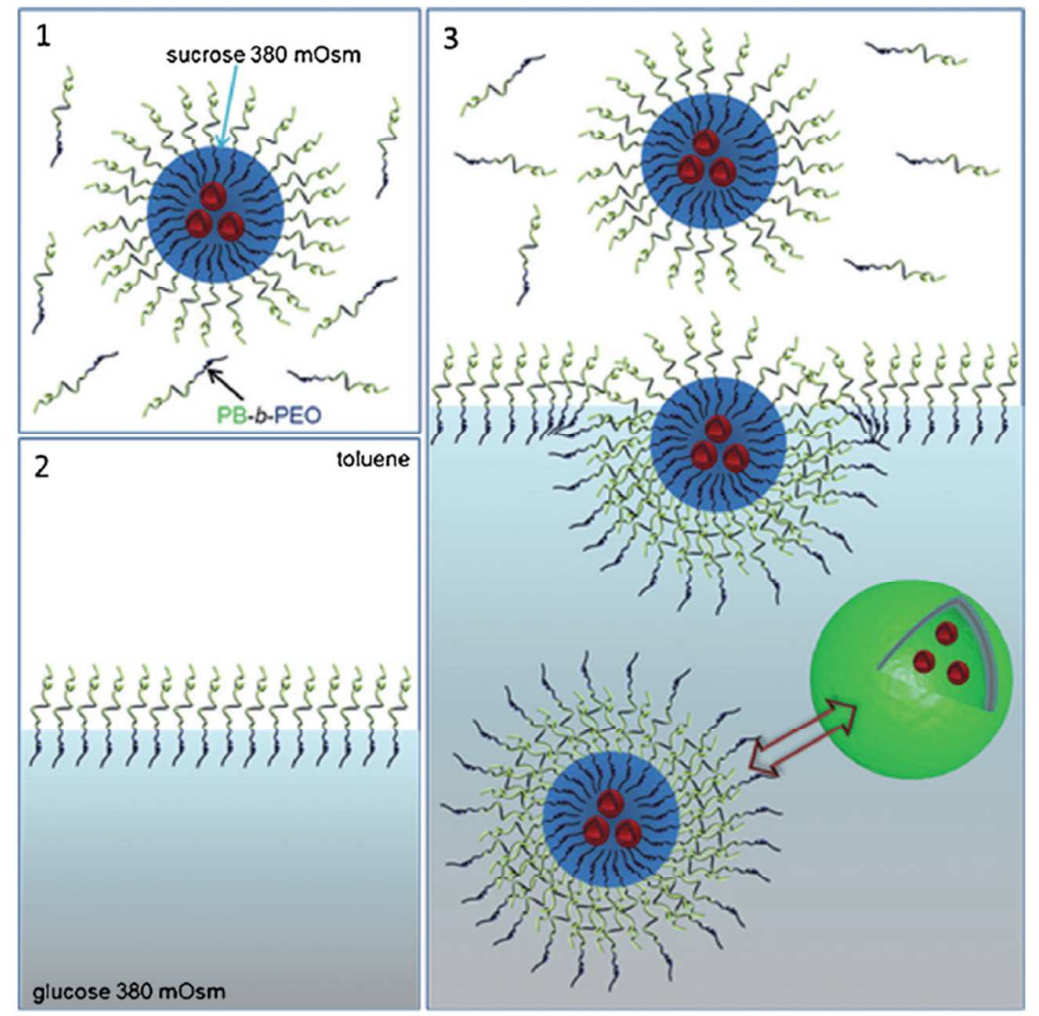

Figure 5. Production of vesicles using the emulsion method result in a layer-by-layer formation of the vesicle. If the first water droplet already contains small polymersomes (red), the production of small vesicles enclosed into larger vesicles (green) is possible using this approach. ${ }^{85}$ (Reproduced with permission from reference 85. Copyright (2012) John Wiley and Sons).

Preparing round droplets (and eventually form polymer nanoparticles) from emulsions is something which was achieved by microfluidics ever since it was invented. ${ }^{87}$ In here, the fluids flow through nanometer to micrometer sized channels and meet at a definite time point, forming their aggregates. Consequently, this approach can also be used to form polymersomes (Figure 6). Initial results by Weitz and Battaglia et al. did not only proof that 
polymersomes can be formed in that way, but that encapsulating drugs can be done in the same step. ${ }^{88}$ The advantage of microfluidics is its high yield of encapsulation which comes with the nature of microfluidics. Each channel hosts one part of the original material which means that the cargo is injected directly into the vesicles. The lack of a consecutive cleaning step is of course a positive point for microfluidics. So far, polymers of various molecular weights have been used not only to make polymersomes, but also to create small polymersomes encapsulated in large polymersomes. ${ }^{89-91}$ With regard to the polymers applied, the methacrylic-based poly(methacrylic phosphoryl choline)-poly(diisopropylaminoethyl methacrylate) (PMPC-PDPA) ${ }^{88}$ as well as the ROP based PEG-PLLA ${ }^{90-92}$ have both been applied, showing that there is in principle no restriction towards the polymers. The examples show further that microfluidics can be expanded from being just a sophisticated tool to an effective emulsion-based method for polymersomes formation. It is now also possible to use it for the production of multicompartment capsules encapsulating polymersomes as already mentioned for the emulsion-based method, ${ }^{86,91,93}$ but also capable of mimicking other techniques. PMPC-PDPA based polymersomes, for example, are created using an in-situ $\mathrm{pH}$ switch, hence a way of solvent switch. ${ }^{88}$ So while the solvent switch is limited to polymers soluble in water-miscible solvents and the emulsion-based way is limited to water-insoluble solvents, microfluidics can do both. As with every method, there are also disadvantages involved in microfluidics. Although scale 
up by numbering up is easy, the slow production speed of vesicles through microfluidics does hinder a larger scale application. It should also be noted that mainly large polymersomes (above $50 \mu \mathrm{m}$ ) have been reported so far when prepared in microfluidics. Furthermore, no further application of polymersomes made by microfluidics (drug delivery or nanoreactors) has been shown so far which is why the scope of this polymersome making method is not clear at the moment. $^{20}$

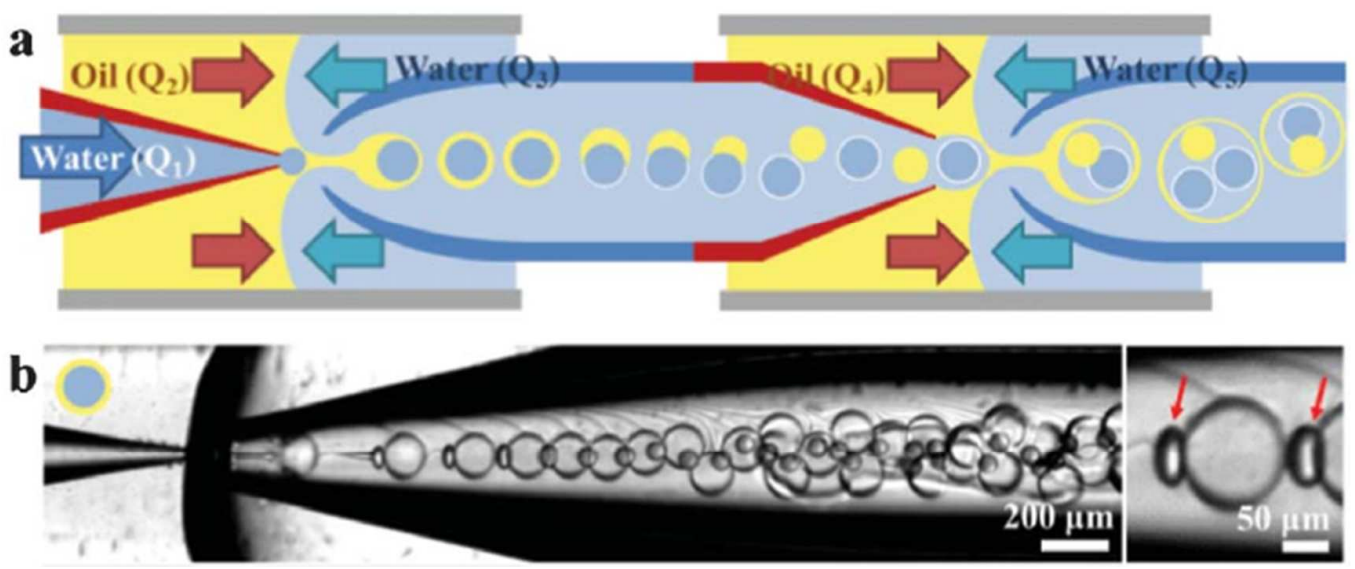

Figure 6. (a) Schematic illustration of the microfluidic device for preparation of multi-compartmentalised polymersomes containing several inner polymersomes. (b) Optical microscope image showing injection of polymersomes into the innermost droplets of double-emulsion drops. For the formation of vesicles the breaking of the middle phase is triggered by the core droplets of the aqueous suspension. ${ }^{89}$ (Reproduced with permission from reference 89. Copyright (2013) Royal Society of Chemistry).

\subsubsection{Formation during Polymerisation}

A very recent method to form polymersomes is to produce them from a hydrophilic macroinitiator, e.g. while the hydrophobic part of the final 
amphiphilic block-copolymer is produced. Armes et al. now showed various examples of this polymerisation-induced self-assembly (PISA). The most important prerequisite for PISA is that the monomers of the hydrophobic part of the final polymer are soluble in water. Due to this solubility switch, an originally homogeneous solution gives an amphiphilic block-copolymer which can then self-assemble in situ (Figure 7). ${ }^{46,94}$ The polymerisation of hydroxylpropylmethacrylate (HPMA) from a macroinitiator is widely reported by the group as the monomer HPMA is water-soluble, but the polymer PHPMA is not. Interestingly, the polymerisation method used by the group, reversible addition fragmentation transfer polymerisation (RAFT) works without a metal catalyst starting from a water-soluble pre-polymer as chain transfer agent (macro-CTA). ${ }^{46,94-95}$ It is not clear so far, whether this is a necessary prerequisite or if other living polymerisation techniques are also feasible for this approach.

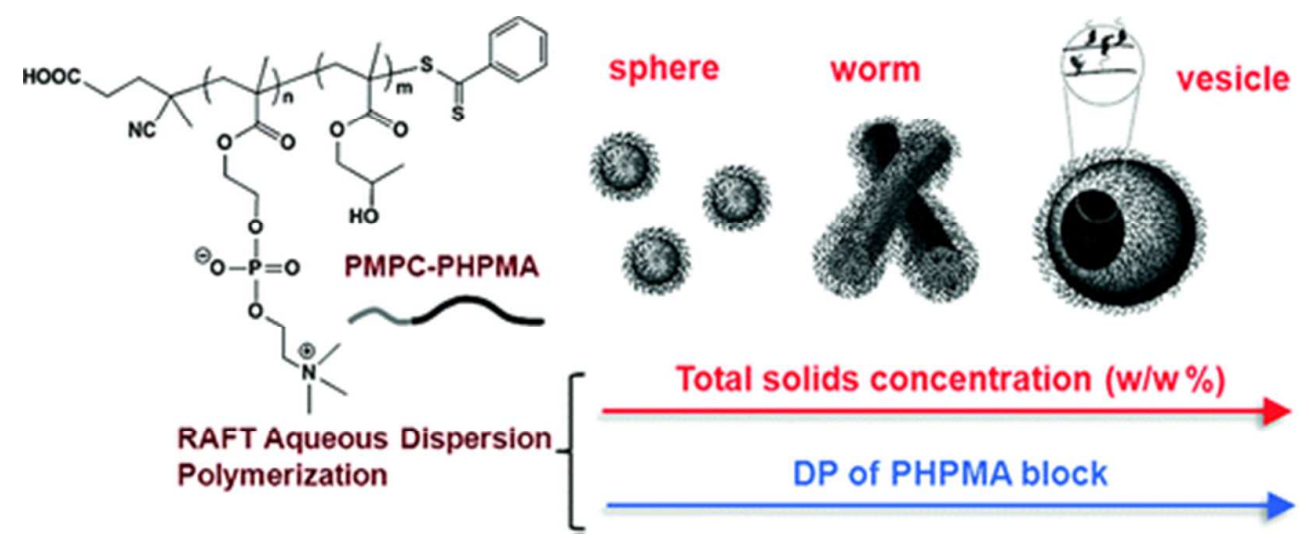

Figure 7. PMPC-PHPMA vesicles are synthesised using PISA. With increasing block length of the building block-copolymer, the self-assembly structures change from spheres 
(micelles) to worms to vesicles. ${ }^{94}$ (Reproduced with permission from reference 94. Copyright (2011) American Chemical Society).

Within PISA, the polymerisation can be stopped at any time point, resulting in the structure that is stable at that specific hydrophilic to hydrophobic aspect ratio. Due to the dynamics in the system during polymerisation, the micelle-polymersome transition hindered for polymersomes formed by solvent switch is allowed for the PISA approach. This was repeatedly used to generate phase diagrams of different block-copolymers showing which self-assembly structure is favoured for which block-copolymer ratio from micelles (or spheres) over worms to vesicles (Figure 8). ${ }^{46,96-97}$
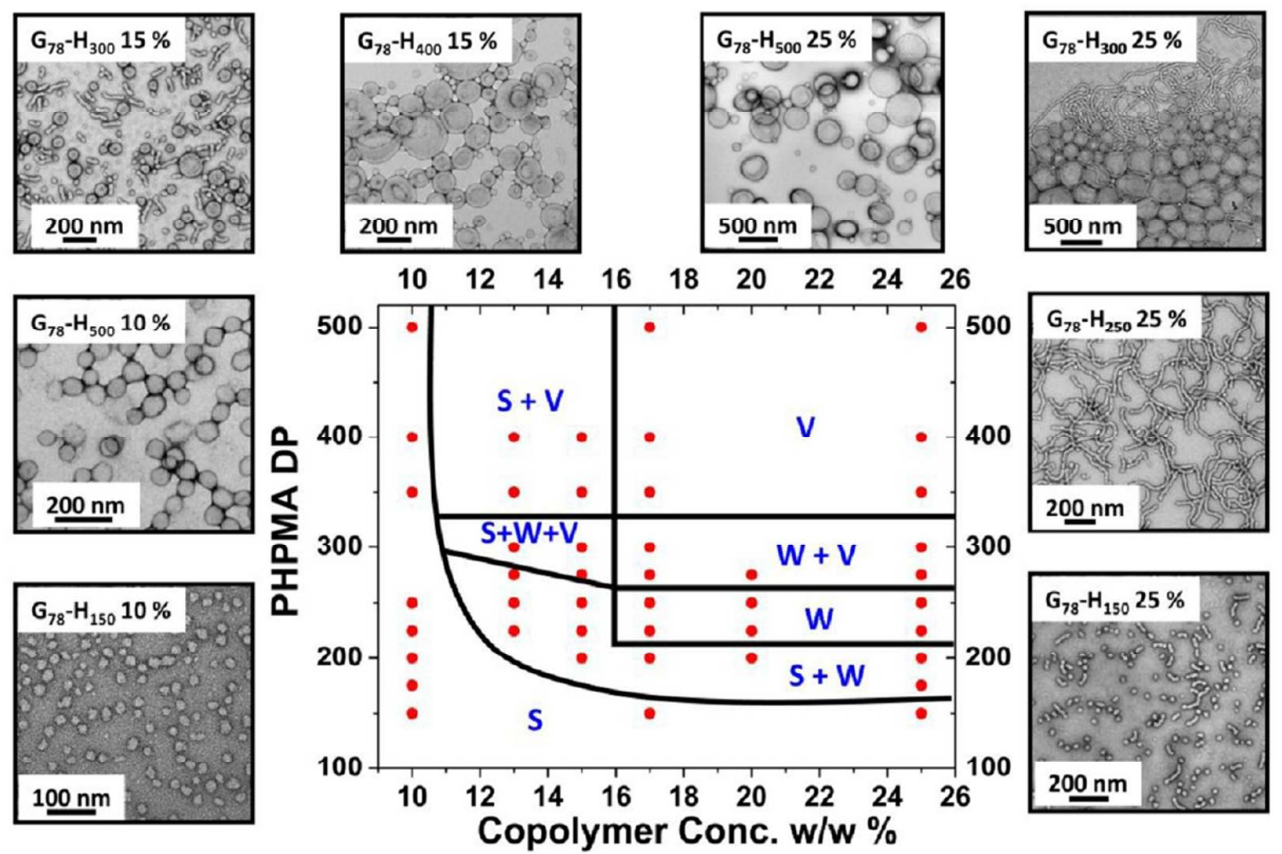
spheres (micelles), worms and vesicles. ${ }^{46}$ (Reproduced with permission from reference 46. Copyright (2012) American Chemical Society).

Once the vesicles are formed, no further transition of the self-assembly structures occurs. However, an ever-growing polymer results in an ever longer hydrophobic block of the block-copolymer. With no phase change occurring, a thickening of the membrane is the only option left for the polymer and this is what happens. It is not obvious from the start in which direction the membrane will be growing. The membrane now either grows to the inside, to the outside, or do both simultaneously. ${ }^{95-96}$ The first option would lead to vesicles of decreasing surface area, but stable size, and the second option to ones with increasing surface area and size and the combination of both growing directions would lead to a slight increase in size and a stable surface area. For their PHPMA based system, Armes et al. report a growth to the inside only, e.g. a decrease in surface area but stable size. Decreasing the surface size means that the inner surface area is shrinking constantly with the hydrophilic part of the polymer getting very crowded on the inner side of the membrane ${ }^{98}$ For this polymer, it is suggested that the constraint block-copolymer chains flip through the membrane so that they are finally on the outside of the vesicle. Due to the convex nature of the outside of the vesicle, this is energetically favoured over a crowded concave inner part of the vesicle. So far, it is not clear whether this is true for other polymerisation achieved with PISA as well. ${ }^{95-96}$ 
PISA is, of course, not limited to water-borne systems only. Whenever a monomer turns from solvophilic to solvophobic during polymerisation, self-assembly to polymersomes and other structures occurs (Figure 9). Armes et al. reported PISA in ethanol using poly(benzyl) methacrylate) (PBzMA) from a poly(acrylic acid) (PAA) macro-CTA. The resulting PAA-PBzMA is then reported to assume all known polymeric self-assembly structures from micelles to vesicles. ${ }^{51}$ Further chain extension with a semi-fluorinated polymer poly((2,2,2-trifluoroethyl) methacrylate) (PTFEMA) gave an $A B C$ triblock-copolymer. Polymersomes of this PISA-approach then showed a definite composition of the inner and outer membrane..$^{50}$ In a very similar approach Lowe et al. also applied PISA in ethanol using 2-phenylethyl methacrylate as the monomer with switching solubility. It should be noted that the group used poly(2-(dimethylamino)ethyl methacrylate) (PDMA) as a starting CTA. ${ }^{52}$ Being a solvophilic block in ethanol, it is rapidly applied as solvophobic block in waterborne polymersomes. ${ }^{99-101}$ Besides in ethanol, PISA was also attempted successfully in n-dodecane with poly(lauryl methacrylate)poly(benzyl methacrylate) [PLMA-PBzMA] again with PBzMA as the switching block. $^{102}$ 
(A)

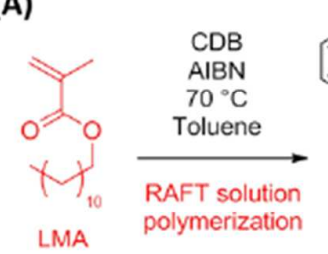

(B)

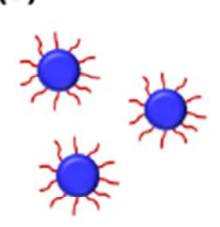

Spheres

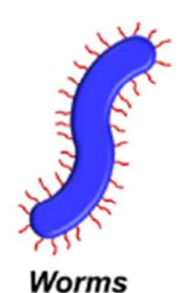

Worms

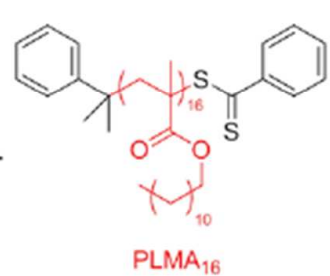

$\mathrm{PLMA}_{16}$

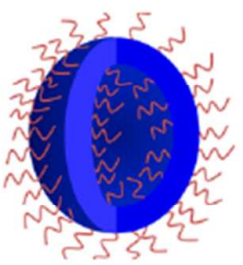

Vesicles

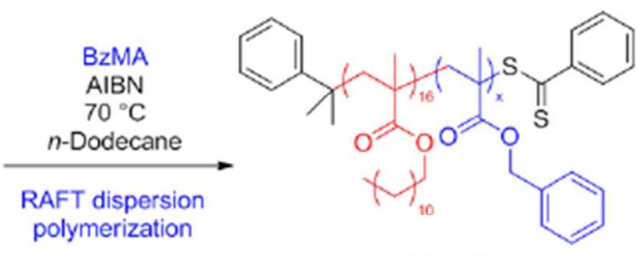

$\mathrm{PLMA}_{16}-\mathrm{PBzMA}_{\mathrm{x}}$

(C)

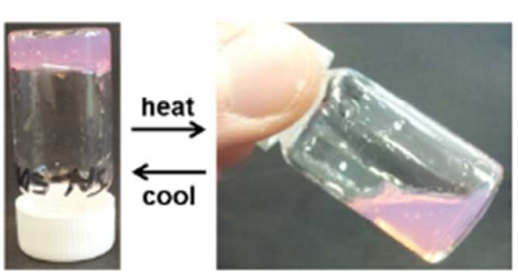

$\mathrm{PLMA}_{16}-\mathrm{PBzMA}_{37}$ in $n$-dodecane

Figure 9. PISA, performed in n-dodecane using poly(lauryl methacrylate)-poly(benzyl methacrylate) (PLMA-PBzMA) as solvophilic and solvophobic parts. ${ }^{102}$ (Reproduced with permission from reference 102. Copyright (2014) American Chemical Society (Creative Commons CC-BY)).

\subsection{Bulk Polymeric Capsules}

Recently, polymeric capsules, with a polymer membrane domain and an empty core domain, as another type of polymeric compartmentalized model are also attracting considerable attention. Differing from above-mentioned polymersome of which the polymer in the membrane should be strictly amphiphilic to form a bilayer structure, the polymer in the polymeric capsules can be varied freely which endow us with more freedom to design different functionalised polymeric capsules as desired introducing also controlled permeability of the membrane and surface functionality. Tailoring morphology 
and physicochemical properties is of great interest to improve and broaden the potential of polymeric capsules for advanced applications in drug delivery, nanoreactor and biotechnology. To date, significant progress has been made in the design and fabrication of such models, and various routes leading to hollow polymer capsules have been explored. Here, only the template-based polymerisation, emulsion polymerisation and distillation-precipitation polymerisation will be discussed. The additionally widely used layer-by-layer approach has been reviewed extensively recently ${ }^{41-44}$ and will not be repeated here.

\subsubsection{Template-Based Polymerisation}

In recent years, the colloidal template method, involving the coating of a sacrificial inorganic/organic template with polymer followed by stabilization of the polymer shell via a cross-linking reaction, is especially popular. ${ }^{6,19}$ The template is typically selected to be a readily removable nanoparticle, e.g., made from silica, gold or other organic compounds. Thus, by combining this method with surface-initiated polymerisation (SIP) technique, an elegant and versatile route to form polymeric capsules with tailored properties has been widely studied. Usually, the polymeric capsule is obtained after cross-linking the shell and removing the core. ${ }^{103-105}$ The obvious advantages of this template-based polymerisation strategy to synthesize the polymeric capsules are: (i) the size of capsules can be easily tuned by choosing different sized 
nanoparticles as templates, (ii) the thickness of the shell can be controlled by controlling the molecular weight of the anchored polymers, (iii) the composition of the polymer shell can be easily varied by using different functional monomers, (iv) and the post-functionalisation on the surface of the polymeric capsules can proceed from the active end-site of the grafted polymer chain. Recently, the rapid development in polymer chemistry ${ }^{106-107}$ laid a solid foundation for the prosperity of SIP in the synthesis of polymeric capsules. In this regards, the pioneering example on this topic was shown by Hawker et al. ${ }^{103}$ who employed surface-initiated controlled nitroxide mediated radical polymerisation (NMRP) to grow polystyrene from the surface of silica nanoparticles and cross-linked the capsules either thermally by the incorporation of vinyl benzocyclobutene groups or chemically by the reaction of maleic anhydride repeat units with a diamino crosslinker. Hereafter, in subsequent publications, surface-initiated atom transfer radical polymerisation (ATRP) was also successfully employed to synthesize polymeric capsules by Fukuda $^{104}$ and Kang et al. ${ }^{105}$ For instance, by surface-initiated ATRP strategy, an oxetane-functionalised block copolymer was built on the surface of SNP. Taking advantage of the ring-opening reactivity of oxetane for the shell cross-linking and dissolving the core, a monodisperse hollow capsule was obtained(Figure 10). ${ }^{104}$ The created almost monodisperse polymeric capsules can form well-ordered close-packed colloidal crystals that diffract light. ${ }^{108}$ Also, based on this surface-initiated ATRP method, Kang and coworkers prepared a 
block copolymer of PS-b-PMMA grafted silica nanoparticle. Ultraviolet exposure of the hybrid particles resulted in cross-linking of the PS intermediate layer, then after the decomposition of the PMMA outermost layer and subsequent removal of the silica core by HF etching provided cross-linked PS hollow capsules. ${ }^{105}$ In the following study, the authors also demonstrated the membrane of the PS hollow capsules can be functionalized by hydrophobic polystyrene with terminal azide groups $\left(\mathrm{PS}-\mathrm{N}_{3}\right)$ and hydrophilic poly(ethylene glycol) with terminal thiol group (PEG-SH) via alkyne-azide and thiol-ene surface click reactions, and produced a hairy PS hollow capsules with binary polymer brushes on the surface. ${ }^{109}$

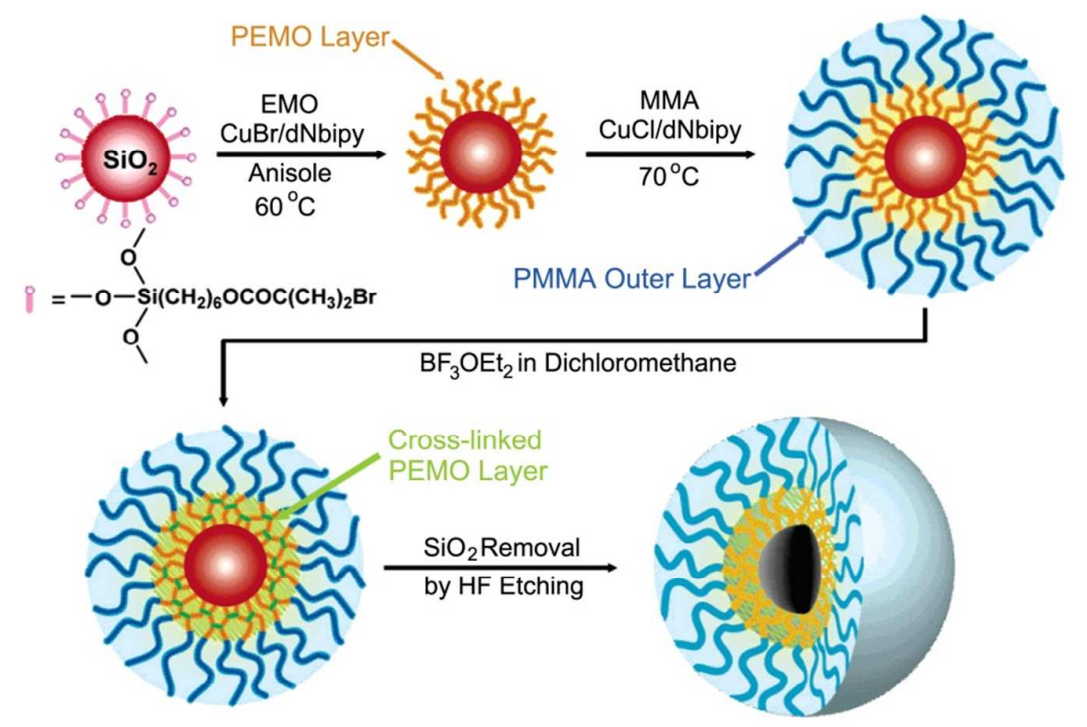

Figure 10. Schematic representation for the synthesis of polymeric capsules by SIP. Employing 3-ethyl-3-(methacryloyloxy)methyloxetane (EMO), and methyl methacrylate (MMA) as monomers. PEMO-b-PMMA grafted silica nanoparticles (SNPs) were obtained via surface-initiated ATRP. After crosslinking the PEMO layer of (PEMO-b-PMMA)-SNP 
by cationic ring-opening reaction of the oxetane groups of the EMO moieties the core is removed by $\mathrm{HF}$ etching. This process gave cross-linked (PEMO-b-PMMA) polymeric capsules. ${ }^{104}$ (Reproduced with permission from reference 104. Copyright (2007) American Chemical Society).

It is well-known that RAFT has emerged as a promising controlled radical polymerisation technique due to its versatility and simplicity, and the advantage that the resulting polymer is free from the contamination of metal catalyst. ${ }^{107}$ Also, RAFT is compatible with almost all of the conventional radical polymerisation monomers. As shown in a recent study by Voit and coworkers, taking advantage of surface-initiated reversible addition-fragmentation chain-transfer (RAFT) polymerisation, polymeric capsules can also be successfully synthesized, and the general procedure is shown in Figure $11 .{ }^{110}$ After anchoring a dithiocarbonate RAFT agent onto the silica nanoparticle, block-copolymers PtBMA-co-PDMIPM- $b$-PHPMA were grown from the surface by RAFT polymerisation. 2,3-Dimethylmaleic imidopropyl methacrylate as a photo-cross-linker was incorporated in the polymer chain to allow crosslinking the shell, and after removal of silica templates by HF buffer solution ( $\mathrm{pH}$ 5.0) well-dispersed polymeric capsules could be obtained. In the following study, an intelligent polymeric capsule with an ultrathin membrane was developed by using a $\mathrm{pH}$ sensitive monomer diethylaminoethyl methacrylate (DEAEMA) as well as a disulphide containing monomer pyridyldisulphide ethylmethacrylate 
(PDSM) as main units. ${ }^{111}$ Cross-linking was successfully achieved by using dithiols of different lengths and led to stable polymeric capsules after removal of the silica template. For this constructed polymeric capsule (PDEAEMA-Co-PPDSM), its permeability of membrane could be well tuned by adjusting the $\mathrm{pH}$ and using different lengths of the cross-linkers. Also, due to the disulphide cross-linker, degradation of the capsules using glutathione as reducing agent can be achieved which is further significantly promoted at more acidic environment. Using a rather long-chain dithiol cross-linker it could be demonstrated that a protein-sized and cationic hyperbranched polymer can successfully go through the cationic membrane in the swollen state which makes this polymeric capsule be a very promising candidate in the application as nanoreactor or nanocarrier. ${ }^{111}$

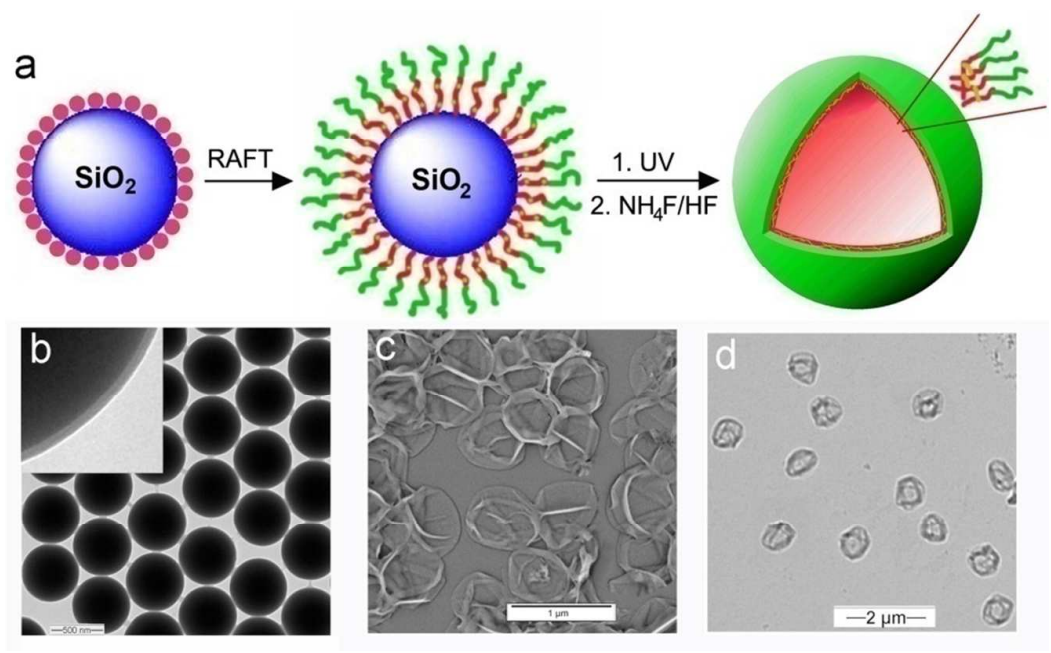

Figure 11. (a) Schematic illustration of the procedure to form polymeric capsules based on the surface-initiated RAFT polymerisation from silica nanoparticles as templates, (b) TEM image of polymer grafted silica nanoparticles (inset is a magnification of (b)), (c) SEM and (d) TEM images of the synthesized polymeric capsules. ${ }^{110}$ (Reproduced with 
permission from reference 110. Copyright (2011) American Chemical Society).

Among these studies, the surface initiating photo-polymerisation has also been widely used as another simple, versatile and effective approach to generate polymeric capsules. ${ }^{112-113}$ As shown in Figure 12, Yin and coworkers immobilised crosslinked PDMAEMA shell containing co-initiator amino groups on the surface of silica particles, which can initiate MMA in the presence of photoinitiator under UV-irradiation. ${ }^{113}$ Polymeric capsules with well-defined outer PMMA shell were obtained after removing the silica cores. It is noteworthy that this surface initiating polymerisation technique is easy and robust for the synthesis of different kinds of polymeric capsules by changing the used monomer. One example shown by Yang et al. was that an optically active polymer can be incorporated into the polymer shell by copolymerisation of optically active N-propargylamide copolymers and maleic anhydride and vinyl acetate. ${ }^{112}$ Accordingly, each constructed polymeric capsule can be considered as a single chiral resolution vessel due to its inner cavity and the helical polymer chains grafted in the shell. Apart from chiral recognition and chiral resolution functions, such polymeric capsules are also expected to show other applications involving chiral catalysis, controlled release, and chiroptical materials. 


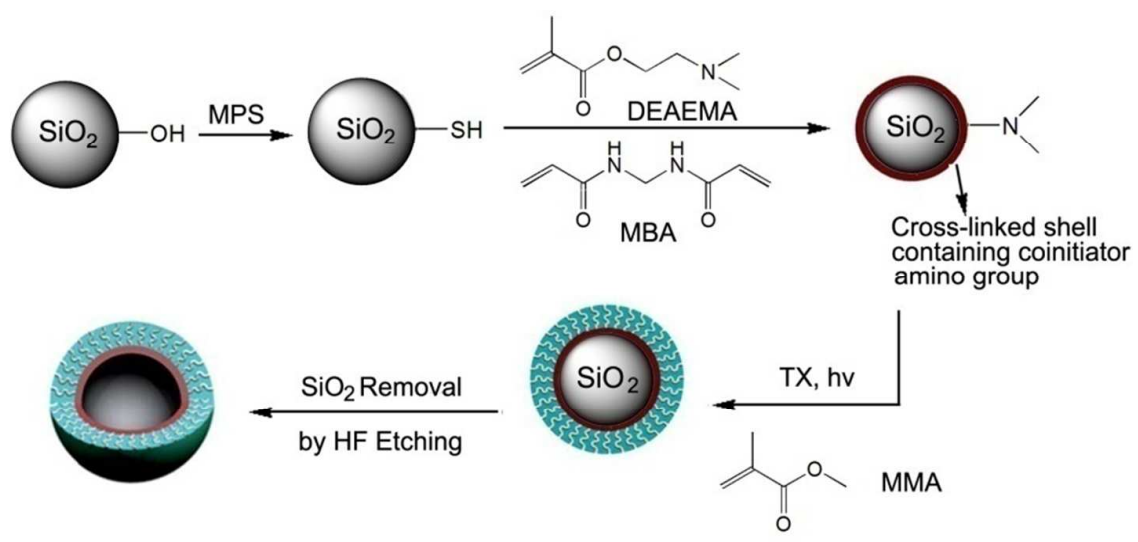

Figure 12. The process for synthesis of polymeric vesicles with well-defined PMMA

brushes from a $\mathrm{SiO}_{2}$ nanoparticle. ${ }^{113}$ (Reproduced with permission from reference 113.

Copyright (2010) Royal Society of Chemistry).

In addition to the above-mentioned "grafting from" method to anchor polymers onto the template surface, Boyer and coworkers showed an approach based on "grafting to" method to anchor polymers onto the surface of gold nanoparticles to synthesize polymeric capsules. ${ }^{114}$ In detail, two different functional diblock polymers were made by RAFT polymerisation with a cross-linkable segment comprised of an alternating copolymer of styrene and maleic anhydride. It has been well confirmed that the polymers synthesized via RAFT polymerisation can be used to assemble around gold nanoparticles via the high affinity of RAFT end-groups for gold surfaces as demonstrated for the first time by McCormick, Lowe, and Sumerlin et al... ${ }^{115-116}$ Accordingly, for the obtained gold nanoparticle polymer brush, after cross-linking of the polymer layer by the addition of ethylene diamine in the presence of 1-ethyl-3-(3-(dimethylamino)propyl)carbodiimide, and the removal of gold 
cores using aqua regia, well-defined polymeric capsules was obtained. Also, the data showed that the use of aqua regia had no negative effect on the integrity of the polymer chains in the shell.

In general, in these discussed template-based polymerisation strategies solid templates such as silica or gold nanoparticles are employed which means harsh chemical reagents (e.g. acids, organic solvents) are required to dissolve the template, ${ }^{103,110,114}$ consequently placing some limitations when chemically sensitive materials such as biomolecules are present. Also, utilisation of solid particles as templates means that the substances of interest have to be encapsulated into the capsule by diffusion after removal of the core, which is inefficient. Taking these issues into account, some soft templates have attracted significant interest such as emulsion droplets or vesicles, which can be readily removed under relatively mild conditions (e.g., aqueous alcohol solutions) and which can be easily pre-loaded with substances. ${ }^{117}$ For example, Ali and coworkers employed vesicles as templates to construct polymeric capsules. ${ }^{118-119}$ As depicted in Figure 13, the approach simply requires the adsorption of short-chain negatively charged copolymers, poly(butyl acrylate-co-acrylic acid) containing a RAFT group, on the oppositely charged unilamellar vesicles of dimethyldioctadecylammonium bromide (DODAB). ${ }^{118}$ Once the RAFT copolymers which were synthesized using the RAFT agent dibenzyltrithiocarbonate (DBTTC), were located on the surface of the vesicle template, chain extension could take place with a monomer feed comprising 
methyl methacrylate, tertiary butyl acrylate and a divinyl crosslinker ethylene glycol dimethacrylate, to form a crosslinked polymer shell. After hydrolysing the tertiary butyl ester groups of the shell, a $\mathrm{pH}$ responsive polymeric capsule was finally obtained. Although, due to colloidal instability, a vesicle template might not be that suitable in the final application, and this soft templating approach still represents a promising method for the synthesis of polymeric capsules due to the mild conditions for "core dissolution" and the easy encapsulation of the substance of interest.

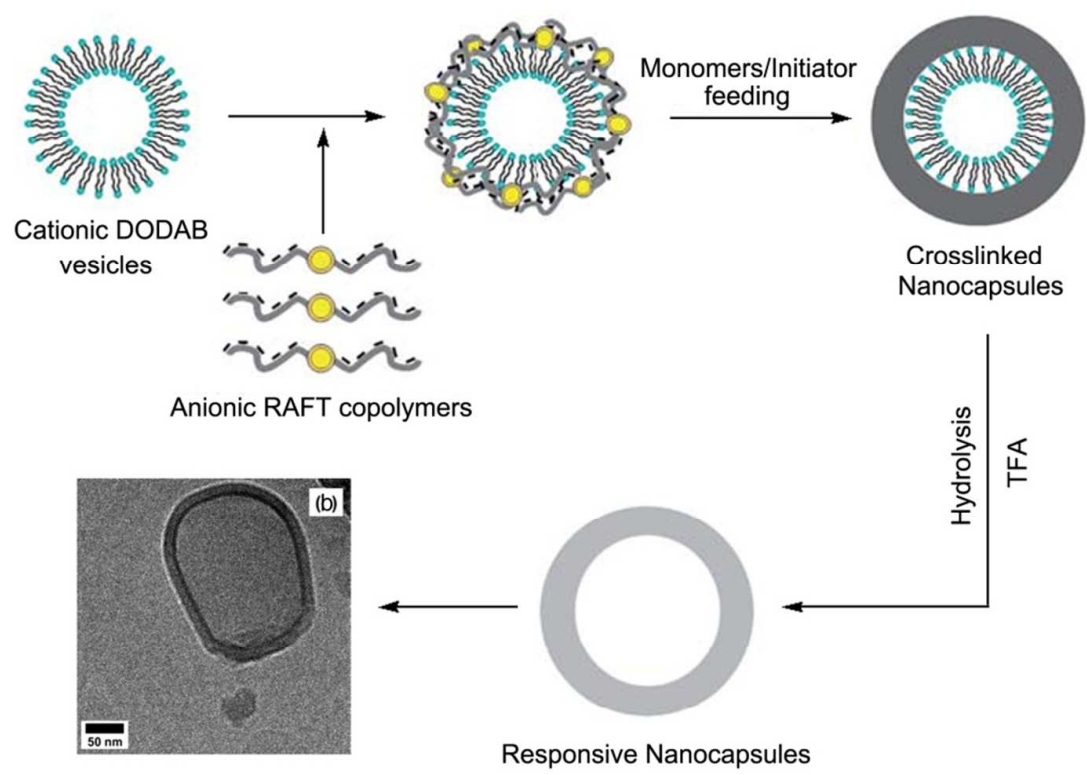

Figure 13. (a) Schematic representation of the synthesis of vesicle-templated $\mathrm{pH}$-responsive nanocapsules by aqueous starved feed emulsion polymerisation using RAFT copolymers as stabilisers, and (b) CryoTEM micrographs of a single synthesized nanocapsule. ${ }^{118}$ (Reproduced with permission from reference 118. Copyright (2011) Royal Society of Chemistry). 


\subsubsection{Miniemulsion Polymerisation}

An alternative approach to generate polymeric capsules, miniemulsion polymerisation, is of special interest and is attracting more and more attention. ${ }^{120-121}$ The method uses a mixture of two immiscible phases and leads to the formation of monodisperse stable droplets either as oil-in-water or water-in-oil miniemulsion in the presence of high shear stress including ultrasonication or high speed homogenizer. The size of the obtained droplets can be varied within the range between 50 and $500 \mathrm{~nm}$ depending on the type and effective amount of the surfactant or co-surfactant. There are almost unlimited choices of monomers/polymers and chemical reactions to generate the polymeric capsules with a hydrophilic or hydrophobic core. Furthermore, the surface of polymeric capsules can be easily functionalised, for example, with biomolecules for specific targeting or biosensing. Therefore, there is no doubt that this technique gives another significant opportunity to produce polymeric capsules with desired properties for a broad range of bioapplications.

Up to now, a host of excellent reports from Landfester's group have shown the versatility of miniemulsion polymerisations as a tool for the fabrication of polymeric capsules composed of different kinds of polymers obtained by a variety of polymerisation types ranging from radical ${ }^{122}$ and anionic ${ }^{123}$ polymerisations to polyaddition ${ }^{124}$ or polycondensation methods. ${ }^{125-126}$ Basically, they can be divided into three categories: 
(i) Formation of polymeric capsules by phase separation:

In phase separation process, normally an organic liquid is chosen as a dispersed phase of the direct miniemulsion which is a solvent for the monomers but a non-solvent for the polymers. By tuning the surface tensions of the participating interfaces in the system, phase separation occurs and the non-solvent is engulfed by the growing polymeric shell, leading to the formation of polymeric capsules with the encapsulated organic liquid in the core(Figure 14). For example, when using the more hydrophobic styrene, the copolymerisation with hydrophilic monomers such as acrylic acid, ${ }^{122}$ methacrylic acid, ${ }^{127}$ or N-isopropylacrylamide (NIPAAm), ${ }^{128}$ a large fraction of capsules could form. Their properties including size and thickness of the shell could be adjusted by changing the ratio of co-surfactant to monomer. In another study, Landfester and coworkers showed a system to encapsulate the volatile fragrance in poly(methyl methacrylate), polystyrene, or acrylic copolymer capsules. When the content of the fragrance was about $25 \mathrm{wt} \%$, a particle morphology was obtained consisting of a matrix composed of the fragrance in the polymer, while only at larger quantities of the fragrance, small microdomains could be clearly formed with homogeneous distribution of the fragrance. ${ }^{129}$ Such varied hollow polymeric capsule morphology was also observed in a system applying the biodegradable surfactant lecithin and the eco-friendly hydrophobe Neobee M5 (triglyceride). ${ }^{130}$ Additionally, nanocapsule formation via the nitroxide-mediated cross-linking 
copolymerisation of styrene and divinyl benzene, stabilised by poly(vinylalcohol) (PVA) to fabricate polymeric capsules was also demonstrated by Zetterlund and Okubo et al.. ${ }^{131}$

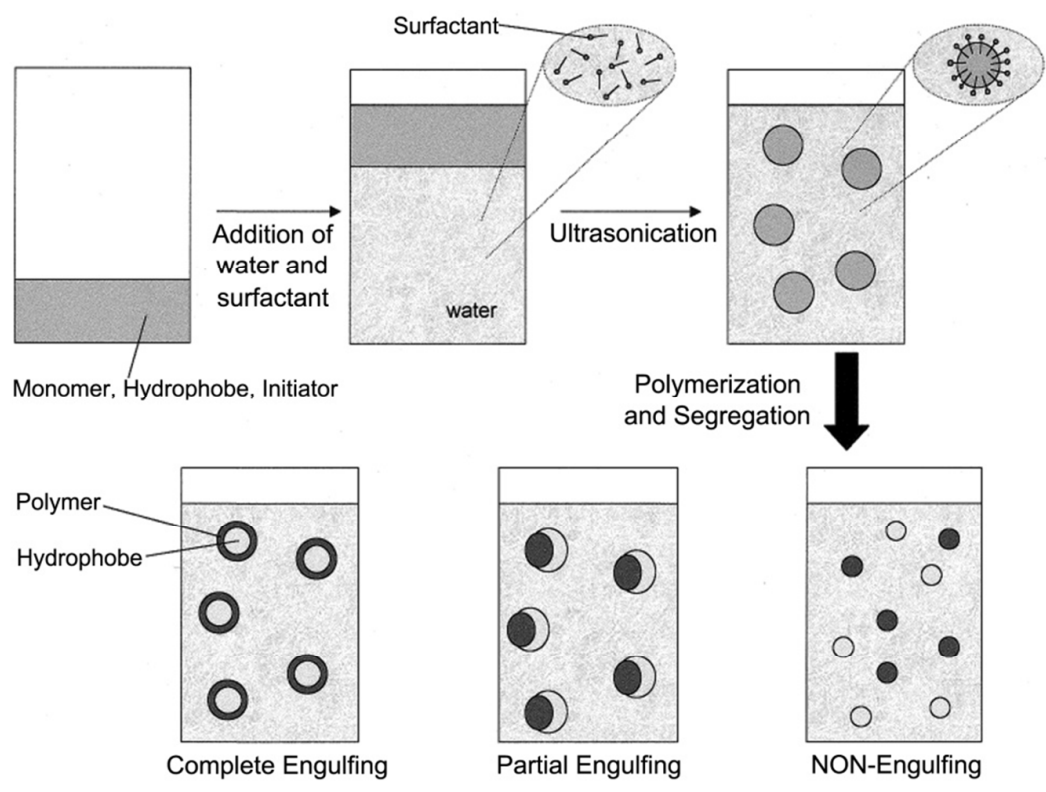

Figure 14. Schematic view of the growing polymeric capsules by phase separation in miniemulsion. ${ }^{122}$ (Reproduced with permission from reference 122. Copyright 2001 American Chemical Society).

(ii) Formation of polymeric capsules by interfacial polymerisation:

Interfacial polymerisation is another highly efficient approach to generate polymeric capsules. On the one hand, this can be realised either by using interfacially active initiators or water soluble initiators generating amphiphilic species, "anchoring" the growing polymeric chain to the interface. On the other hand, this can be also realised if the hydrophilic monomer is present in the 
aqueous phase and the other hydrophobic monomer in the organic phase, and these monomeric species only meet and react to give polymers at the interface. By far, three types of polymerisation strategies including interfacial controlled radical copolymerisation ${ }^{132-133}$, anionic polymerisation ${ }^{134}$, and interfacial polyaddition reactions are mostly used to synthesize polymeric capsules. $^{125,135-136}$

One representative for the formation of polymeric capsules by interfacial controlled radical polymerisation process is to use styrene for the encapsulation of isooctane and potassium peroxodisulfate as anchoring agent. ${ }^{137}$ The formation of a polymeric capsule morphology, especially that of a liquid core and a polymeric shell, was extremely dependent on both the type of RAFT and the initiator. Different RAFT agents lead to different polymerisation rates, thus resulting in different chain lengths as a function of time. In the study, the two exploited RAFT agents, phenyl 2-propyl phenyl dithioacetate (PPPDTA) led to very fast polymerisation reactions, while phenyl 2-propyl dithiobenzoate (CDB) retarded the polymerisation reaction. This could influence viscosity and consequently chain mobility and therefore could cause a deviation from the desired morphology. Also, the type of the used initiator which can influence the surface activity of entering oligomers, was another important factor in obtaining the correct structure. Using PPPDTA living polymerisation with KPS and AIBN, only with KPS the polymeric capsules could be generated. In the case of CDB and KPS, no polymeric capsules could be generated, since the viscosity of the 
growing polymeric shell was probably not high enough to reduce the chain mobility sufficiently, also allowing diffusion of the growing polymer into the droplet.

Taking advantage of an amphiphilic poly(acrylic acid)-block-polystyrene RAFT agent as a surfactant and polymerisation mediator, Luo and coworkers demonstrated that highly uniform polymeric capsules could be obtained. ${ }^{138-139}$ While, instead of acrylic acid, the authors also incorporated the monomer maleic anhydride into the oligomer RAFT agent. ${ }^{140}$ Given that the ammonolysed oligomer RAFT agent would self-assemble on the interface of water/droplets, such self-assembly property combining with the RAFT living polymerisation chemistry rendered the polymer chains to grow inwards gradually in particles, leading to the formation of a polymer shell (Figure 15). Accordingly, via tuning of the hydrophilicity of the oligomer RAFT agent by the structure of oligomer RAFT agent or the degree of ammonolysis, the final morphology of the synthesized polymeric capsules can be easily varied. ${ }^{140}$ In this study, when the molar ratio of ammonia to anhydride groups was 0.9 and with $0.5 \mathrm{wt} \%$ SDS as a co-surfactant, optimised polymeric capsules were obtained. Also, the incorporation of anhydride groups in polymer shell offers great opportunities to functionalise the surface properties of the capsules. 


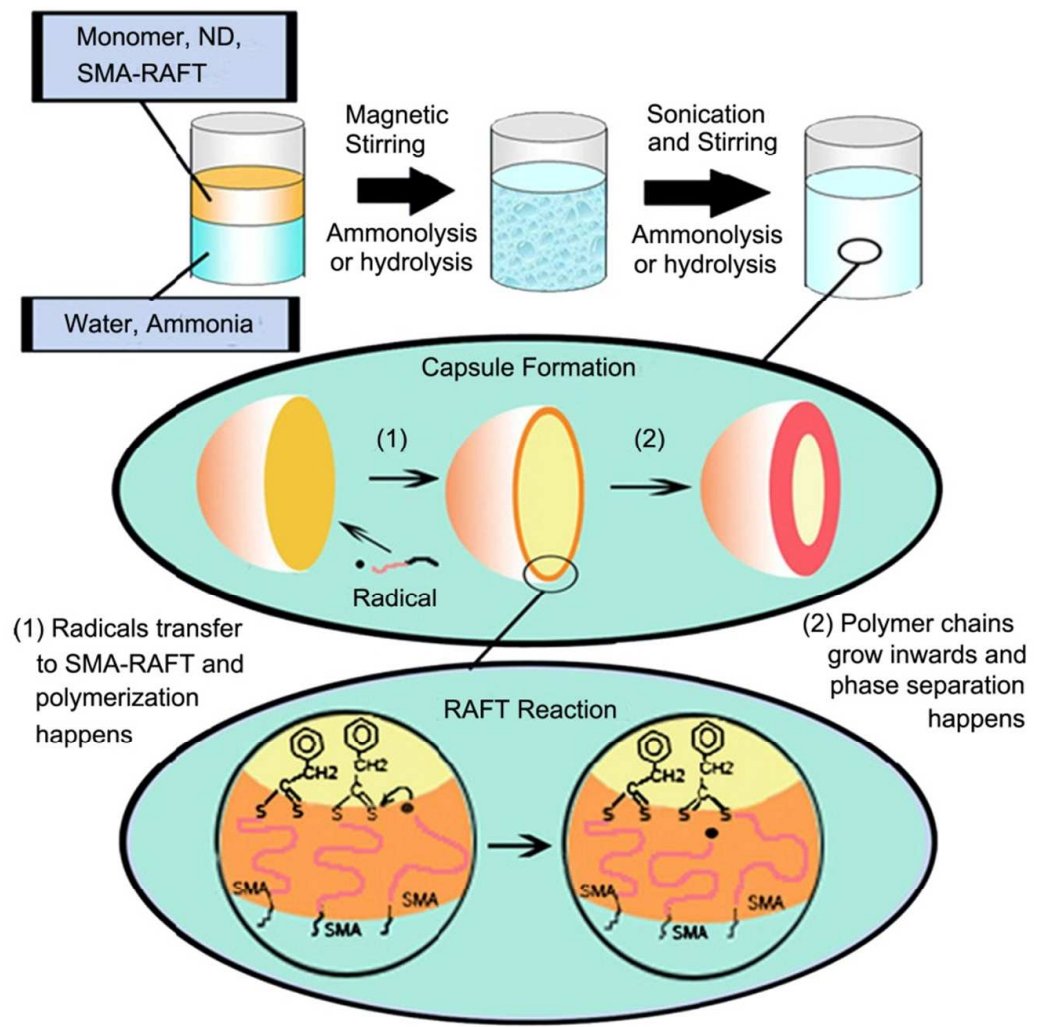

Figure 15. Schematic illustration of the procedure to generate polymer capsules based on RAFT interfacial miniemulsion polymerisation by using an amphiphilic RAFT oligomer (SMA-RAFT) as a surfactant. ${ }^{140}$ (Reproduced with permission from reference 140. Copyright(2007) Elsevier Ltd.).

Matyjaszewski and coworkers ${ }^{141}$ also demonstrated the synthesis of polymeric capsules through an interfacially confined copolymerisation of a monovinyl monomer and a divinyl cross-linker via an activator generated by electron transfer atom transfer radical polymerisation (AGET ATRP) in a miniemulsion system using an amphiphilic block-copolymer poly(ethylene oxide)-b-poly(n-butyl methacrylate) (PEO-PBMACl), as a stabiliser and macroinitiator. The average diameter of the resulting capsules was about 
200-300 nm with good stability and dispersion in some common organic solvents. The introduction of various degradable cross-linking agents into the system resulted in the formation of polymeric capsules that were cleaved under specific conditions.

Besides the RAFT and ATRP polymerisation, anionic polymerisation can also be carried out at the droplet's interface to generate polymeric capsules with encapsulated either aqueous or organic phases as core. ${ }^{120,134}$ In both cases, the reaction is exclusively initiated at the droplet's interface, employing alkyl cyanoacrylates as a monomer. Since the combination of two electron-withdrawing groups (ester and nitrile) bonded to the same carbon atom renders the alkyl cyanoacrylate highly reactive, the interfacial anionic polymerisation of alkyl cyanoacrylate monomers is a very efficient way to obtain biodegradable capsules. In this regard, poly(alkyl cyanoacrylate)-based capsules with different payloads including paclitaxel, ${ }^{142}$ antiepileptic drugs ${ }^{143}$ and insulin ${ }^{144}$ have been demonstrated.

In addition, polymeric capsules could also be synthesized through interfacial polyaddition or polycondensation reactions by using hydrophilic and hydrophobic precursors, which were dissolved in two different (continuous or disperse) phases and reacted at the droplets interface. For example, Landfester and coworkers demonstrated the synthesis of polyurea, polythiourea, or polyurethane capsules with an aqueous core performing the interfacial polycondensation reactions in an inverse miniemulsion 
system. ${ }^{125,135-136,145}$ In this case, the size of the capsules, the thickness and functionality of the shell were studied thoroughly. The size of the capsule was dependent not only on the concentration of the surfactant but also on the time of addition of the second monomer. The thickness of the wall could be controlled by the amount of monomers employed. The functionality of the shell was governed by the nature of the monomers and the ratio between the two monomers introduced in the miniemulsion. Also after cross-linking, the polymeric capsules can be easily transferred into the aqueous phase, whereby the non-reacted diisocyanate groups (from a cross-linker) will hydrolyse, resulting in the formation of primary amine groups on the capsule's surface. Thus further modification of the capsule's surface was realised with targeted molecules such as folic acid, ${ }^{146}$ oligomannose ${ }^{147}$ or antibodies. ${ }^{148}$

(iii) Formation of capsules by precipitation of polymer on preformed droplets:

Recently, via the controlled polymer nanoprecipitation onto inverse miniemulsion droplets, the so-called nanoprecipitation method was developed by Landfester and coworkers which was also able to be exploited to fabricate polymeric capsules. ${ }^{149-150}$ The deposition of a polymeric shell, e.g., PMMA, PCL or PMA, from the organic continuous phase onto the stable nanodroplets dispersed phase was achieved by changing the gradient of the solvent/nonsolvent mixture of dichloromethane/cyclohexane under mild evaporation. After re-dispersion of the nanocapsules in an aqueous medium, 
stable polymeric capsules with a chlorohexidine digluconate loaded aqueous core re-dispersed in the aqueous continuous phase were obtained for the first time. $^{149-150}$

In all, miniemulsion as a tool for the formation of polymeric capsules is one of the most commonly exploited techniques, especially given its versatility, simplicity, productivity, and ease of application on small scales. Moreover, the characteristic feature in the miniemulsion process is that stable, small, and narrowly distributed droplets are formed which could retain ideally identical throughout the polymerisation process. This enables the possibility for the encapsulation of different materials, ranging from liquid to solid, from organic to inorganic, and from molecularly dissolved to aggregated species into polymeric capsules. There is no doubt that such technique provides a wide platform for the construction of various novel functional nanomaterials as required.

\subsection{Proteinosomes}

Very recently, being rather different from polymersomes and polymeric capsules, a new type of membrane-bounded capsule model based on the spontaneous interfacial assembly of amphiphilic recombinant proteins ${ }^{17}$ or protein-polymer conjugates has been reported. ${ }^{16}$ For example, the protein-polymer conjugates, which consisted of about three polymer chains per protein, assembled at water droplet/oil interfaces into micrometer-scale 
water-filled capsules termed proteinosomes. The proteinosomes were delineated by a closely packed monolayer of conjugated protein-polymer building blocks that could be crosslinked such that the micro-compartments were transferred into water without fragmentation (Figure 16). As a consequence, a wide range of guest molecules from small molecule fluorescence dyes to colloidal particles ( $\sim 00 \mathrm{~nm}$ in diameter) could be encapsulated at high efficiency inside the micro-compartments. Moreover, the protein-polymer membrane was sufficiently elastic and robust to withstand partial dehydration and rehydration, and remained structurally intact when held at a temperature of $70{ }^{\circ} \mathrm{C}$ for $90 \mathrm{~min}$, suggesting that the proteinosomes could provide a useful protocell model system for studying the microscale confinement of thermophilic enzymes, or for developing artificial cells capable of undertaking temperature cycling procedures, such as PCR-mediated amplification of entrapped genetic polymers.

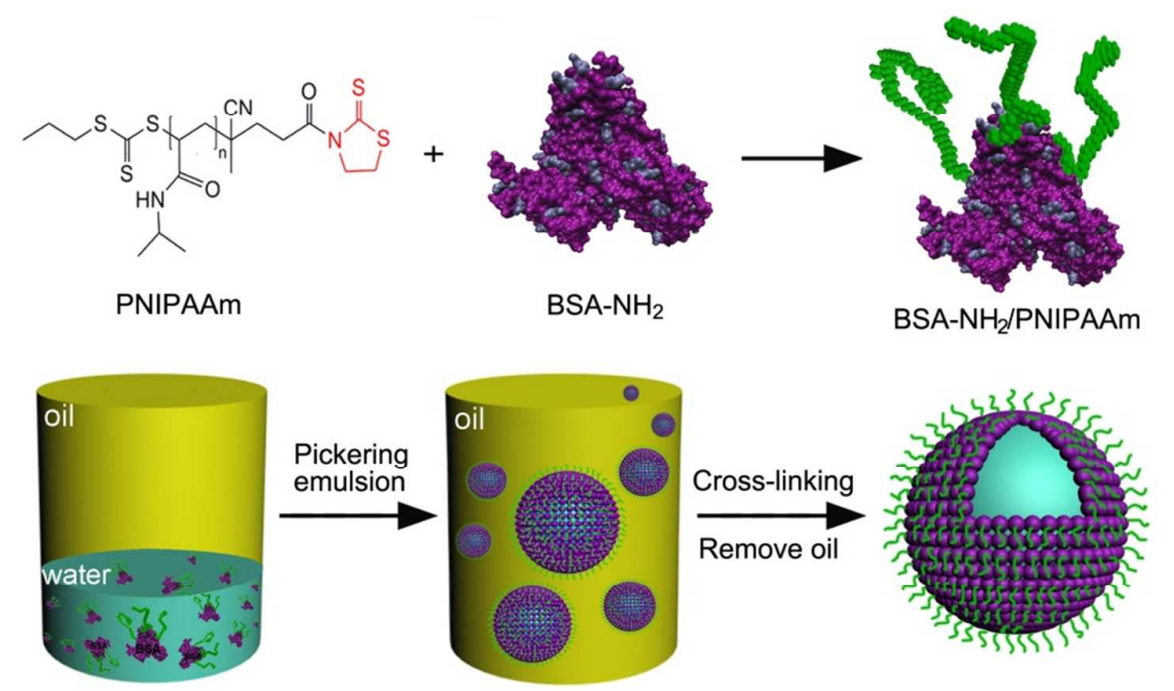

Figure 16. Schematic illustration of a general procedure for preparation of proteinosomes.

44 
Top row: coupling of activated PNIPAAm polymer chains with primary amine groups of cationised BSA-NH$H_{2}$ to produce protein-polymer nano-conjugates (BSA-NH ${ }_{2} /$ PNIPAAm). Bottom row: Use of protein-polymer building blocks for the spontaneous assembly of proteinosome micro-compartments in oil, and their transfer into a bulk water phase. ${ }^{16}$ (Reproduced with permission from reference 16. Copyright (2013) Nature Publishing Group).

A key advantage of the proteinosome system lies with the ability to rationally design the structure and function of the proteinosome membrane by controlling the chemistry of the protein-polymer nanoscale building blocks. This makes the system an attractive candidate for the development of a range of functional proteinosomes. For example, recent studies indicated that the proteinosome membrane was semipermeable, stimulus-responsive, enzymatically active, and mechanically elastic. ${ }^{16}$ It also exhibited size-selective permeability, only allowing molecules less than $40 \mathrm{kDa}$ in molecular mass to enter or leave the proteinosome interior. Moreover, conjugation of the temperature sensitive polymer, poly(N-isopropylacrylamide) (PNIPAAm) to the protein molecules generated a thermally gated system that operated via the swelling or deswelling (at $\mathrm{T}>33^{\circ} \mathrm{C}$ ) of the polymer chains present on the outer surface of the proteinosome membrane. As a consequence, enzyme-mediated peroxidation within the proteinosomes of a small molecule initially present in the external solution was switched on or off by thermally gating the diffusion of 
the substrate through the polymer shell barrier layer (Figure 17). ${ }^{16}$
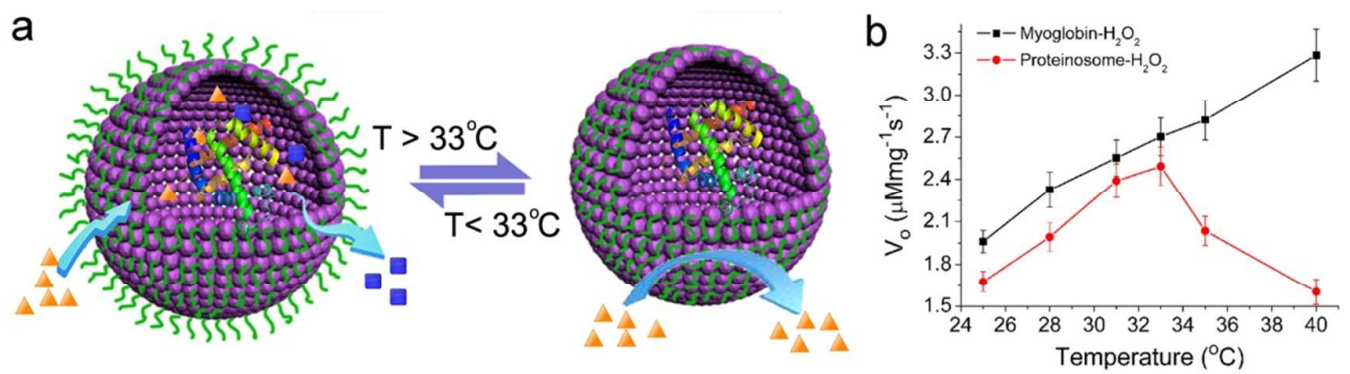

Figure 17.(a) Scheme showing polymer chain gating of enzyme reactions inside proteinosomes. (b) The corresponding temperature-dependent catalytic reaction rates of free myoglobin (black lines) and proteinosome-encapsulated myoglobin (red lines) in the presence of both reactants (2-methoxyphenol and $\left.\mathrm{H}_{2} \mathrm{O}_{2}\right) .{ }^{16}$ (Reproduced with permission from reference 16 Copyright (2013) Nature Publishing Group).

As a further extension of these ideas, the construction of a proteinosome suited for a membrane-mediated cascade reaction was demonstrated. ${ }^{151}$ Three cascade enzymes, glucose amylase (GA), glucose oxidase (GO) and horseradish peroxidase (HRP) have been used to prepare protein-PNIPAAm amphiphilic nano-conjugates to construct proteinosomes comprising an enzyme triad capable of undertaking a multi-step membrane-mediated cascade reaction using starch as the starting substrate. ${ }^{151}$ The tandem reaction can be regulated by the positional assembly and spatial separation of the three enzymes within the membrane of the proteinosome (Figure 18), as 
well as by temperature-dependent changes in the conformation of the covalently attached polymer chains located on the external surface. Furthermore, in a recent work, proteinosomes with new types of higher-order functionality based on controlled membrane disassembly, internalised assembly of a cytoskeletal-like matrix, and self-production of a membrane-enclosing outer wall have been described. ${ }^{152}$ DNA strands were encapsulated in the proteinosomes and their release was triggered by protease-mediated hydrolysis or chemical cleavage of the membrane building blocks. In addition, enzyme-mediated amino acid dephosphorylation was used to generate a supramolecular amino acid hydrogel within the interior of the proteinosome. As a consequence, the mechanical properties of the proteinosome were considerably enhanced. For example, the spherical morphology of the hydrogel-containing proteinosomes was quickly re-established after the protocells were mechanically deformed or deflated due to dehydration. Interestingly, prolonged growth of the encapsulated hydrogel resulted in penetration of the amino acid nanofilaments through the porous shell of the proteinosome to produce a continuous, protease-resistant outer wall that totally enclosed the protein-polymer membrane, ${ }^{152}$ analogous in some respects to the cell wall/cell membrane layered structure of bacterial cells. 

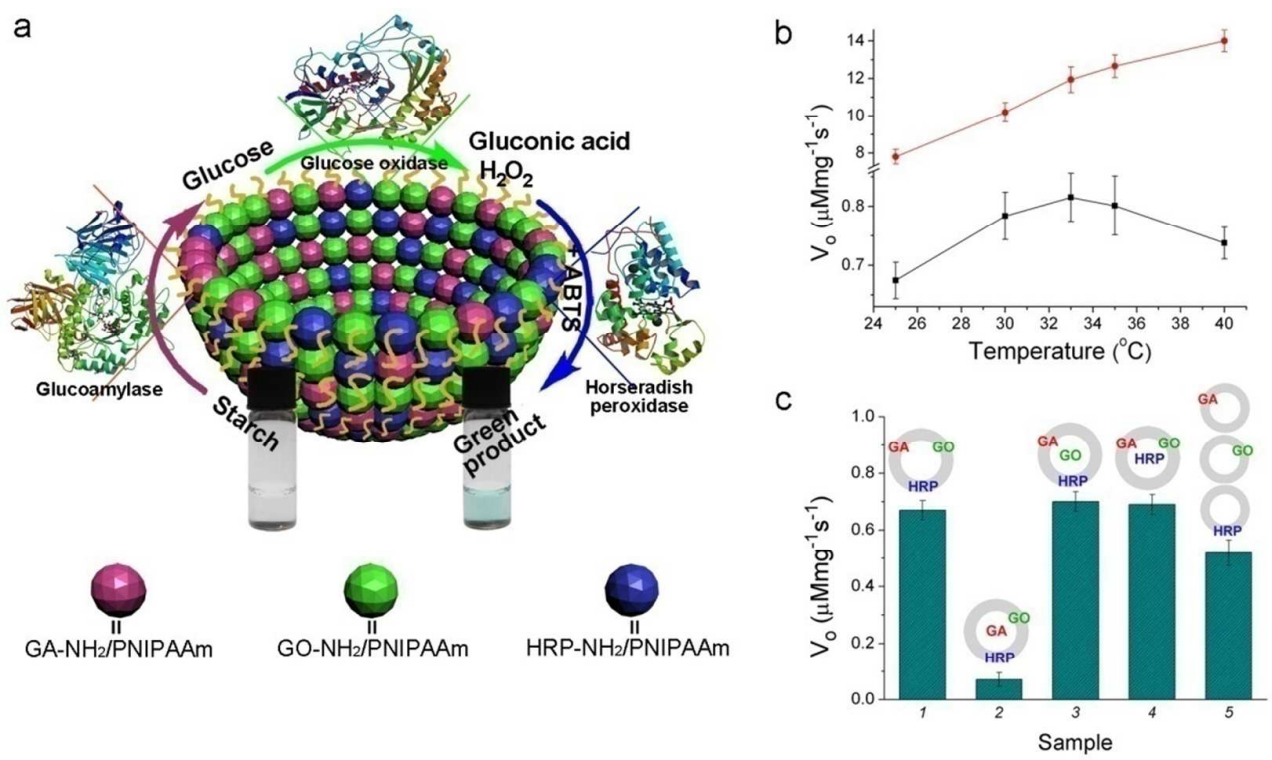

Figure 18. (a) Schematic illustration showing a proteinosome membrane-mediated three-stage cascade reaction in water by using three enzymes, glucose amylase (GA), glucose oxidase (GO) and horseradish peroxidase (HRP), as building blocks. (b) Plots of temperature-dependent initial three-step cascade reaction rates $\left(V_{0}\right)$ (red line: aqueous mixture of GA, GO and HRP enzyme-polymer nanoconjugates; black line dispersion of multi-enzyme proteinosomes comprising membrane building blocks) and (c) summary chart of the catalytic activity of enzyme-polymer proteinosomes prepared with different membrane and internal spatial organisations. ${ }^{151}$ (Reproduced with permission from reference 151. Copyright (2014) Royal Society of Chemistry).

\section{Engineering Functional Polymer Capsules Towards Smart}

\section{Nanoreactors}

\subsection{Membrane-Based Functionalisation of Polymer Capsules}

\subsubsection{Channel Proteins as Transporters}

The improved stability of polymersomes over liposomes does become an

48 
issue when it comes to membrane permeability. Especially polymersomes designed as nanoreactors need to have some way to make their enclosed active cargo like an enzyme accessible without destroying the vesicle. Natural and artificial liposomes contain channel proteins to gate transmembrane traffic, making them an obvious choice also for polymersomes. ${ }^{4,83,153}$

First trials in this direction have studied whether a natural protein will have the ability to retain its structure and with this its functionality in an artificial environment. Initial trials were done with polymer-fortified liposomes which were strengthened by cross-linked poly(butyl methacrylate) (PBMA). ${ }^{154}$ The protein, the bacterial outer-membrane-protein $\mathrm{F}(\mathrm{OmpF})$, continued to gate the activity of beta-lactamases and proved that natural proteins can continue to work in an artificial membrane made from polymers. ${ }^{154}$

It was then a logical consequence to incorporate trans-membrane-proteins into the membranes of polymersomes. From a polymer point of view the soft poly(dimethylsiloxane) (PDMS) as hydrophobic part in combination with poly(methyloxazoline) is applied repeatedly and nearly solely for transmembrane protein incorporation. ${ }^{34,155}$ The block lengths of the polymers vary, but all have a polymersome forming ratio as proven by TEM micrographs. The proteins inserted range from simple pore proteins ${ }^{31,156-158}$ to receptors ${ }^{159}$ and pumping proteins. ${ }^{160}$ Because channel-proteins are amphiphilic, they may influence the shape, although the aim is of course that the polymersomes remain as vesicles. Walz et al. tested this with varying amounts of Aquaporin Z, 
a channel protein, incorporated into the block-copolymer membrane. ${ }^{156}$ From 15 polymer chains per protein onwards, vesicles were formed. When more protein was present, the vesicular shape started to break up and more complex structures were formed. These include planar membranes and smaller vesicles. However, the protein remained active until it started to crystallise at a $1 / 1$ protein to polymer ratio. ${ }^{156}$ It should be noted that proteins generally also insert into polymer bilayer membranes, which do not form vesicles, e.g. as shown for alpha hemolysin in a solid-substrate supported PDMS-PMOXA-PDMS membrane. ${ }^{161}$

Another important aspect to consider is the orientation of the protein. Especially if the protein does only transport in one direction, the orientation becomes of great importance to control specific inward and outward transport. Meier et al. tested this by including Aquaporin 0 into vesicles of the ABA polymer mentioned above and an $A B C$ triblock-copolymer of PEG-PDMS-PMOXA. The authors used polymers with different aspect ratios to ensure the production of vesicles with PEG on the outside as well as ones with PMOXA on the outside, known from previous studies. ${ }^{162}$ Whichever polymer is supposed to go to the inside of the vesicle is considerably shorter than the one going to the outside as it needs to cover less volume on the concave side of the membrane. In a comparison also with lipid membranes, only vesicles from asymmetric $A B C$ triblock-copolymers resulted in a directed insertion of the Aquaporin 0 examined. They could even show that vesicles 
with the opposite membrane structure (PEG-PDMS-PMOXA from outside to inside as compared to PMOXA-PDMS-PEG from outside to inside), also yield the opposite channel protein insertion into them (Figure 19). ${ }^{163}$
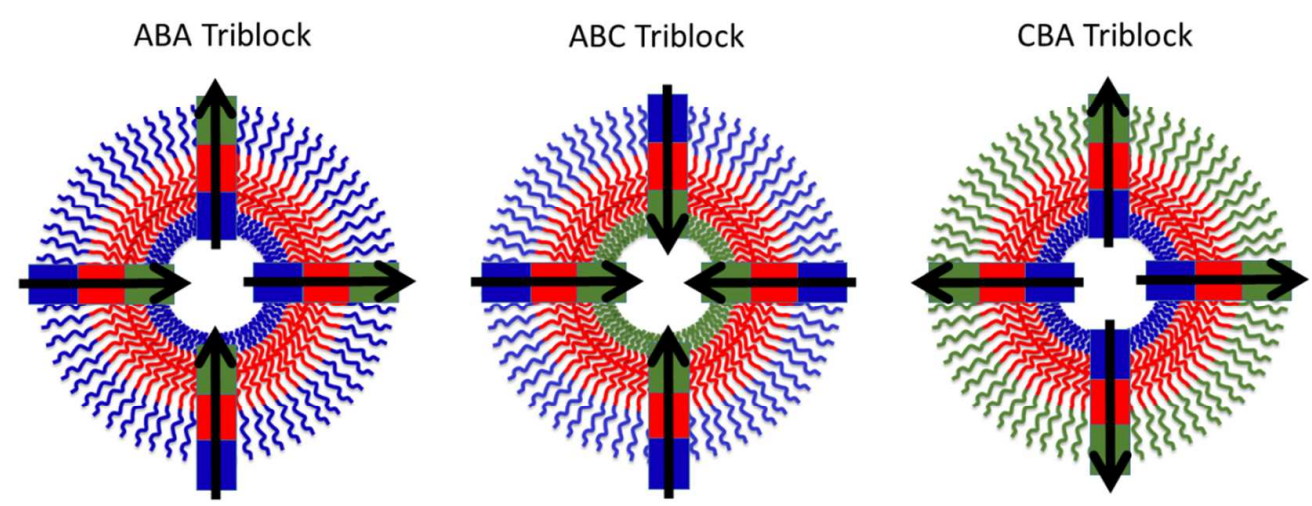

Figure 19. Polymersomes with symmetric (ABA) and asymmetric $(A B C)$ triblock copolymer membranes which hold a channel protein. The protein inserts either with a prominent orientation for the asymmetric $\mathrm{ABC}$ triblock copolymers and at random for the symmetric ABA block copolymers. ${ }^{163}$

Polymersomes with channel proteins were also brought into service as nanoreactors or other functional nanocarriers. Generally it has to be stated that all applications of channel protein-equipped polymersomes were done with the symmetric PMOXA-PDMS-PMOXA ABA triblock copolymer, hence without any control of protein orientation. One of the first examples from Meier et al. shows the insertion of LamB, which is not only a transmembrane protein, but also acts as a receptor for the bacteriophage lambda. Once docked to the receptor, the phage is now able to empty it itself and transfer its cargo (DNA) into the polymersome. ${ }^{159}$ The same group later showed that vesicles including Aquaporin $Z$ had a significantly higher permeability towards ions than plain 
polymersomes. Both systems were exposed to a positive ion gradient and only the vesicles including the channel proteins reacted towards this change. Van Gelder et al. conducted a study where OmpF was inserted into the membrane, but the group went a step further and also included the ViVax nucleoside hydrolase $(\mathrm{TvNH})$ into their vesicles as it can activate prodrugs based on riboside. ${ }^{164}$ A number of such prodrugs were now inserted and the riboside was cleaved off. It should be noted that both examples rely on a non-oriented insertion of channel proteins to ensure transport in and out of the vesicles and to avoid the build-up of osmotic pressure from the inside as diffusion barrier. ${ }^{164}$ Montemagno et al. managed to achieve a significant milestone by rebuilding a vesicle that can produce ATP. ${ }^{160}$ The polymer, PEtOxa-PDMS-PEtOxa is very similar to the methyl analogue discussed so far, so it does not pose a significant change, especially as it is also well characterised. ${ }^{165}$ The significance of this work is that the authors needed to include bacteriorhodopsin (BR) to establish the proton gradient, but also the ATPase to use that very gradient for the production of ATP. It is remarkable that the authors used a symmetric block-copolymer and thus lacked control over protein orientation. The ATPase has the rotating outer bit, which may be too bulgy to insert the "wrong" way, but only nanoreactors where a vast majority of BR is inserted correctly will work, as the wrongly inserted BR proteins would reduce the proton gradient built up by the other proteins. The authors were able to show that the nanoreactor is working as intended and does produce 
ATP from ADP and phosphate. ${ }^{165}$ Despite this achievement, nothing is stated on the yield of these nanoreactors, which may explain why this work was not continued so far.

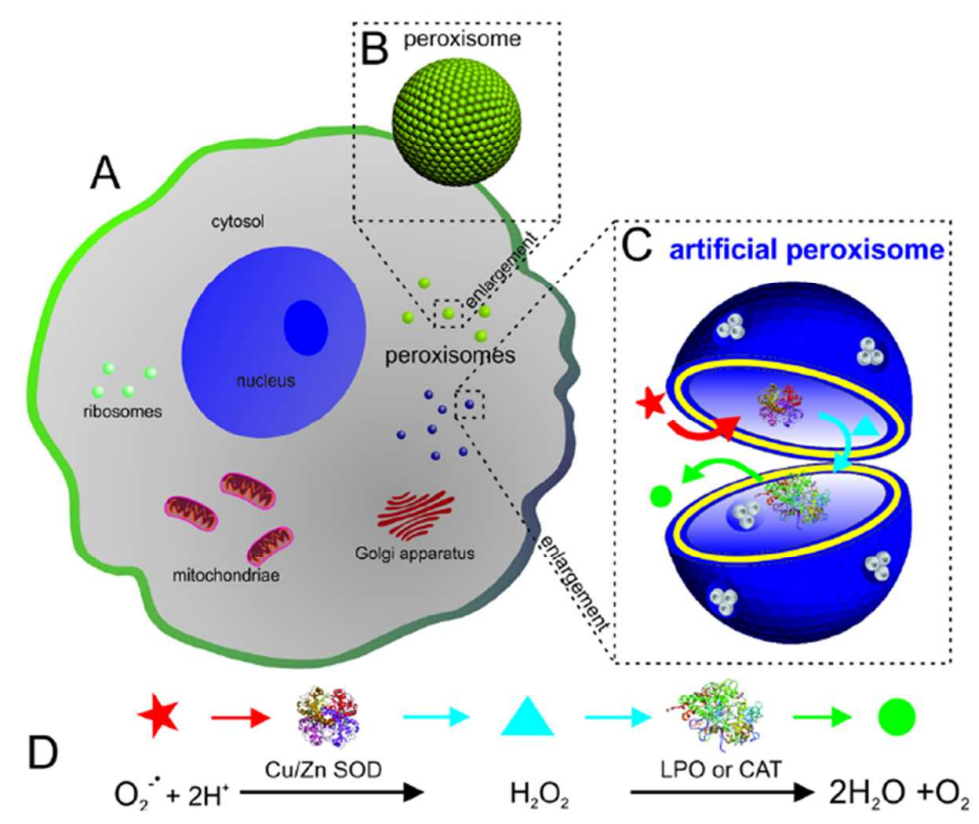

Figure 20. An artificial peroxisome (C) containing $\mathrm{OmpF}$ as a channel protein and enclosed proteins to act as nanoreactors within the cell $(A)$. The polymersomes aid the natural peroxisomes $(B)$ naturally present in the cells. The separate reaction steps are shown in part D. ${ }^{157}$ (Reproduced with permission from reference 157. Copyright(2013) American Chemical Society).

Hunziker and Palivan et al. did a series of consecutive studies on polymersomes of the ABA block-copolymer (PMOXA-PDMS-PMOXA) with the channel protein OmpF to create a biologically functional nanoreactors. Their final goal was the creation of a synthetic organelle, specifically an artificial 
peroxisome, e.g. vesicles that clear its surroundings of superoxides. ${ }^{158,166-167}$ As proof of concept, acid phosphatase was included in the polymersome. The phosphatase is too big for the OmpF pores, so it stays inside, but its substrates and products can enter and leave the vesicle. ${ }^{31}$ Amplex Red was a suitable substrate as its dephosphorylated version is fluorescent, while its phosphorylated version is not. In a sense, this nanoreactor was rightfully described as $\mathrm{pH}$ sensitive, simply because the acid phosphatase is only active at acidic $\mathrm{pH}$ values. However, the pores are functioning independent of the $\mathrm{pH}$ value, hence the $\mathrm{pH}$ does not gate the entrance to the vesicle. ${ }^{31}$ The proof of concept was not a peroxisome as yet, but a first one was achieved late without transmembrane proteins, exploiting the limited permeability of the plain polymersomes towards very small molecules like hydrogen peroxide. ${ }^{167}$ The authors now had a proof of concept of a OmpF-based nanoreactors and an initial peroxisome. Both concepts were then combined to yield a far better functioning peroxisome than the initial one. The group equipped the vesicles with a complex system of proteins inside and the OmpF in the membrane. First of all, the stress-inducing Xantine was oxidised by Xantine Oxidase and the product then reacted further to hydrogen peroxide with the aid of $\mathrm{Cu} / \mathrm{Zn}$ and superoxidase. Hydrogen peroxide would still be toxic, so a third enzyme, lactoperoxidase, then transformed it into water and oxygen. ${ }^{166}$ This artificial peroxisome proved to work extracellular but also within cells. As a last step, this nanoreactors was now not only included in cells but the HeLa cells were 
also treated to experience oxidative stress. When the cells were previously treated with the artificial peroxisomes mentioned, they could withstand stress levels that proved to be toxic to untreated cells. ${ }^{158}$ To the best of our knowledge, this represents the first example of polymersomes as artificial organelles within a cell that actually aid the cells survival using enclosed enzymes as protected drugs. This marks a significant milestone in combining the applications of polymersomes as nanoreactors and drug delivery systems. (Figure 20)

\subsection{2 pH Responsive Polymersomes}

One of the easiest ways to produce a responsive polymersome is to include a $\mathrm{pH}$ sensitive monomer in the hydrophobic block. Upon change in $\mathrm{pH}$ this part becomes hydrophilic, leading to a then entirely hydrophilic block-copolymer. Such a polymer does not form polymersomes anymore, inducing a disassembly of the vesicles. ${ }^{20,168-169}$ Not only is the $\mathrm{pH}$ easy to control and can be changed once the polymersome is formed, it is also known to change during biological processes like endocytosis. The latter one makes $\mathrm{pH}$ sensitive polymersomes perfect candidates of drug delivery systems, which is widely reported. ${ }^{170-171} \mathrm{~A} \mathrm{pH}$ responsive polymer for polymersomes can, of course, be responsive towards a basic $\mathrm{pH}$ (e.g. boronic acid) $)^{33}$ or towards the acidic region (e.g. tertiary amines) ${ }^{66,76,172}$ For some amine-based $\mathrm{pH}$ sensitive polymersomes, the term $\mathrm{CO}_{2}$-sensitive polymersome is also used in 
literature. ${ }^{173-174}$ In these examples the addition of $\mathrm{CO}_{2}$ is used as a means to drop the $\mathrm{pH}$ of the solution, e.g. exploiting the formation of carbonic acid leading to a protonation of the substrate. Albeit the addition of $\mathrm{CO}_{2}$ being a neat way to change the $\mathrm{pH}$ of the solution, the polymers are responding to the $\mathrm{pH}$ change of the solution and are thus $\mathrm{pH}$-responsive rather than $\mathrm{CO}_{2}$-responsive.

With respect to $\mathrm{pH}$ sensitive polymersomes, it can be stated that all of them are produced by controlled radical polymerisation and thus are based either on acrylates, methacrylates or other vinyl-based polymers with a respective side chain. The reason for this is quite simple, because $\mathrm{pH}$ sensitivity comes along with protonation and thus a lone electron pair exists. This lone pair could act as an initiator in ring opening polymerisation (ROP) or anionic polymerisation, making these tough methods to introduce $\mathrm{pH}$ sensitivity or any functionality with a lone electron pair and require at least the use of protecting groups in corresponding monomers. ${ }^{175}$ This has only been accomplished so far with trityl-protected polyhistidine, where after deprotection PEG-polyhistidine block-copolymers were self-assembled into $\mathrm{pH}$-sensitive polymersomes (Figure 21). ${ }^{176-177}$ The pKa of these polymersomes and whether disassembly or a conformation change is reached with decreasing the $\mathrm{pH}$ value, can be tuned if some poly(lactic acid) is polymerised alongside the polyhistidine in the hydrophobic block. ${ }^{178}$ 
Figure 21. Synthesis of PEG-PHis via an NCA-based Ring-Opening-Polymerisation with a consecutive deprotection (cleavage of trityl group) of the polyhistidine. ${ }^{177}$

The vast majority of $\mathrm{pH}$ sensitive polymersomes responds to a change towards an acidic medium, e.g., are amine-based. However, van Hest et al. did also report a system based on poly(styrene boronic acid) (PSBA)- which responds towards a change into basic $\mathrm{pH}$ (Figure 22). ${ }^{33}$ Being a Lewis acid, the boronic units of the $\mathrm{PEG}_{22}-b-\mathrm{PSBA}_{93}$ bind a hydroxyl ion in a basic $\mathrm{pH}$ range. The negative charges present are responsible for solubility switch and the polymersome disassembles. For the system reported, the pKa is between 9 and 10. 


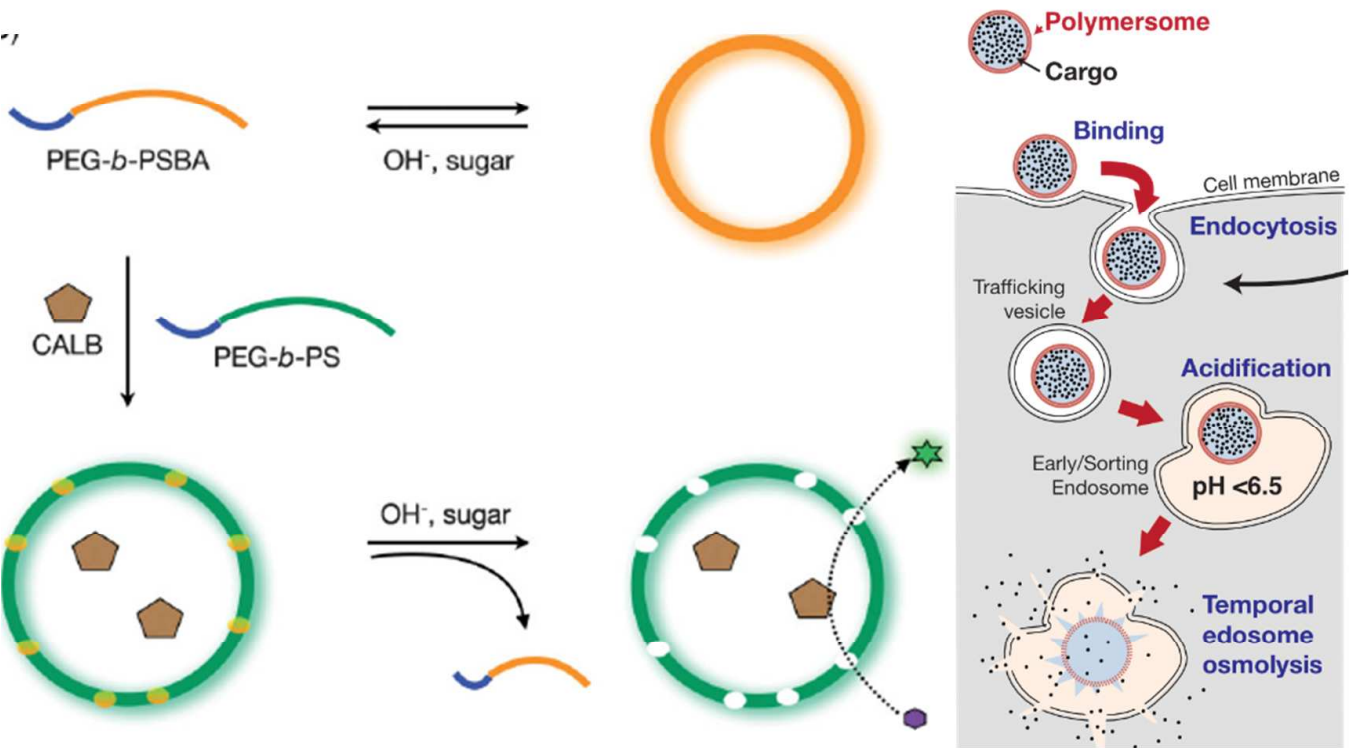

Figure 22. $\mathrm{pH}$ sensitive polymersomes using PEG-PS and PEG-PSBA (structure in Table at the end of the Review) as nanoreactors for enzymatic reactions where the membrane can only be passed at $\mathrm{pH} 9$ or lower (left). ${ }^{33}$ PDPA-containing $\mathrm{pH}$ sensitive polymersomes used as drug-delivery-systems exploit the endosomal $\mathrm{pH}$ switch to release their cargo (right, redrawn from reference with permission). ${ }^{179}$ (Left part reproduced with permission from reference 33. Copyright (2009) John Wiley and Sons; right part reproduced with permission from reference 179. Copyright (2009) Royal Society of Chemistry).

The tertiary amines used in $\mathrm{pH}$ sensitive block-copolymers are mainly attached to a methacrylic backbone. A variety of authors use poly(diisopropylaminoethyl methacrylate) PDPA $^{13,172,180-183}$ or its ethyl (PDEA) $)^{56-57,66,76,111,184-187}$ and methyl (PDMA) ${ }^{52,100,188}$ derivatives. It should be noted that a change in the substitution from isopropyl units towards methyl units causes a significant change in the pKa of the polymer. While PDPA has a pKa of 6.4 at room temperature, ${ }^{13}$ that of PDEA is already at $7.3^{10,58}$ and PDMA 
has a pKa of about $7.5^{10,58}$ at room temperature. PDMA is also temperature sensitive, thus a dual-responsive polymer, but this dual property has only been applied rarely so far by Lecommendoux et al.. ${ }^{99}$ Both other derivatives are used extensively by Battaglia et al. (PDPA) and Voit et al. (PDEA) in their respective polymersome systems. PDPA has been proven to be a perfect candidate for drug delivery applications. It is used to exploit the $\mathrm{pH}$ drop occurring after endocytosis in the early and late endosomes. The polymersomes are stable in cell media as well as the bloodstream $(\mathrm{pH} \mathrm{7.4)} \mathrm{but}$ disassemble in the early endosomes ( $\mathrm{pH}$ 6.0) and release their payload at this stage (Figure 22). What happens to the single polymer chains is not known so far. It should be noted that polymersomes of this specific polymer system of Battaglia et al. (POEGMA-PDPA) are able to cross the blood-brain-barrier, thus enabling polymersomes of POEGMA-PDPA to deliver drugs to the brain, an especially protected part of the body.

On the other hand, polymersomes containing PDEA proved to have a pKa suitable for application as nanoreactors, especially cross-linked ones. In contrast to drug delivery systems, enzyme-based nanoreactors need to be operational at close-to-neutral $\mathrm{pH}$ values to avoid denaturation of the enclosed enzyme at low pH. In their cross-linked state (maleimide-based cross-linker), the polymersomes show a reversible swelling upon hitting the pKa. It means that the disassembly process of the polymersomes is hindered by the cross-linking bonds leaving a swollen and permeable polymersome. ${ }^{66}$ It should 
be noted that the "switching point" of these polymersomes decreases with increasing cross-linking density. The PEG-PDEA-PDMIBM polymer applied by Voit et al. to form cross-linked nano reactors shows a pKa of 7.0 (Figure 23). ${ }^{186}$ The authors showed that single, but also cascade reactions can be controlled with these nanoreactors. In all examples, reactions take place at an acidic $\mathrm{pH}$ only, when the membrane is swollen. When the membrane was in a non-swollen state (basic $\mathrm{pH}$ ), no reaction was observed. ${ }^{186-187}$ Again with a fully reversible $\mathrm{pH}$ switch at 7.0 , the same group applied very similar polymersomes (with a benzophenone cross-linker) also as drug delivery system. ${ }^{76}$ The drug, doxorubicin, is released in different speeds depending on the cross-linking density and $\mathrm{pH}$ and also targeting towards specific cell types has been introduced. An application on cells did not only proof it being non-toxic but the drug effect was also proven on different cell types. ${ }^{189}$ 

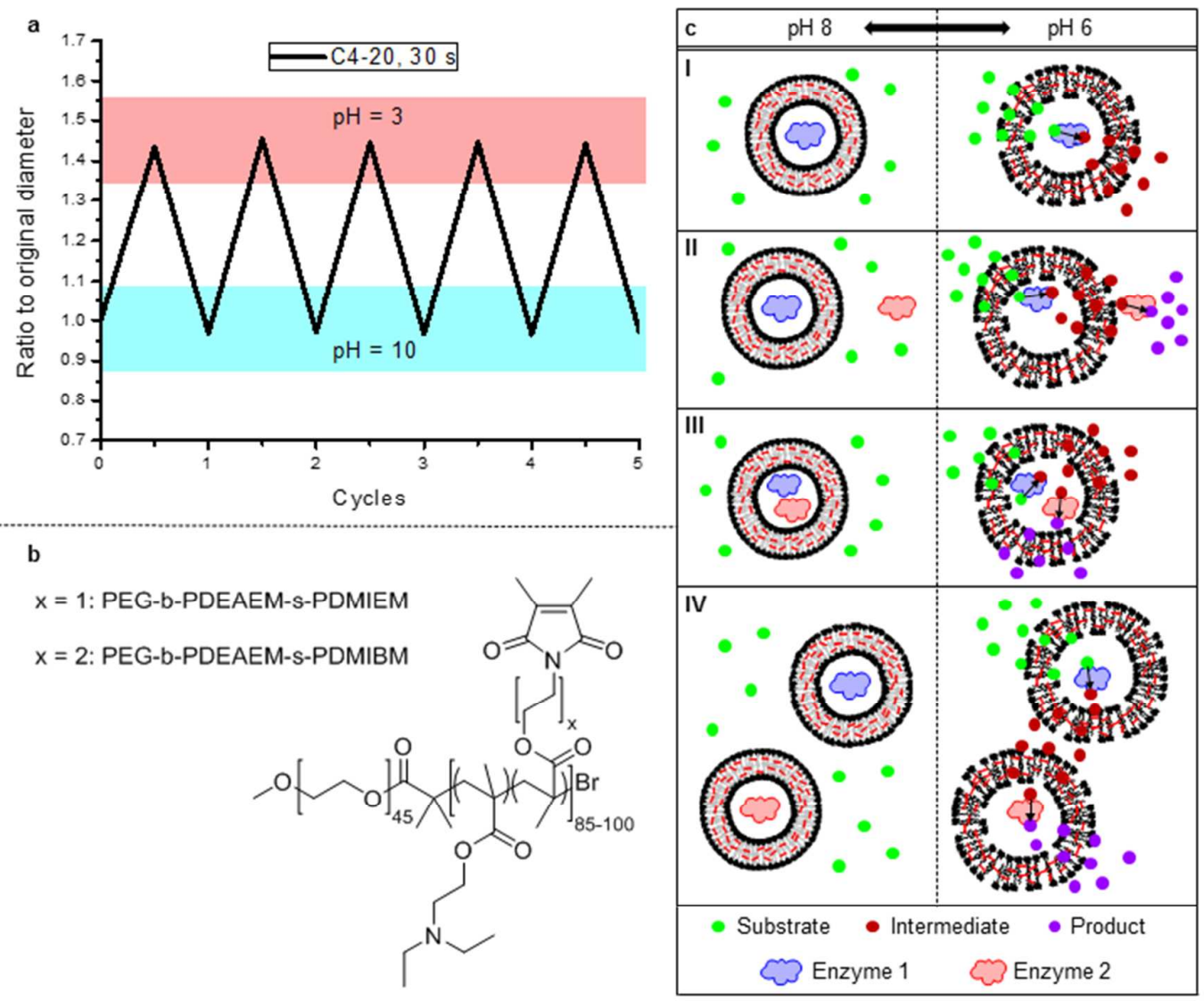

Figure 23. Cross-linked and $\mathrm{pH}$ sensitive polymersomes show a reproducible swelling-deswelling behaviour (a) ${ }^{186}$ from a bifunctional PEG-PDEA-s-PDMI(E/B)M block-copolymer (b). The vesicles allow for single and cascade enzymatic reactions, also across separate vesicles (c). ${ }^{183}$

Apart from the system just discussed, there are a number of other polymersomes with a reversible swelling behaviour ( $\mathrm{pH}$ induced) reported. The main difference is that other systems use physical cross-linking with polystyrene as reported by Eisenberg et al. (Figure 24$)^{184}$ rather than chemical cross-linking. Using this glassy polymer, the adhesive forces in the polystyrene 
part are stronger than the repulsive forces resulting from the protonation. The polymersome stays intact, but does show a swelling ("breathing" as described by the authors) as already discussed for cross-linked vesicles. When van Hest et al. mixed their PEG-PSBA with PEG-PS, they saw a similar effect. Just with 10 w\% of PEG-PSBA in the mixture the adhesive forces of PS were strong enough to keep the polymersome intact. Again, the change in $\mathrm{pH}$ leads to a swelling of the vesicle, which the authors exploit to gate enzymatic activity inside the polymersome. ${ }^{33}$

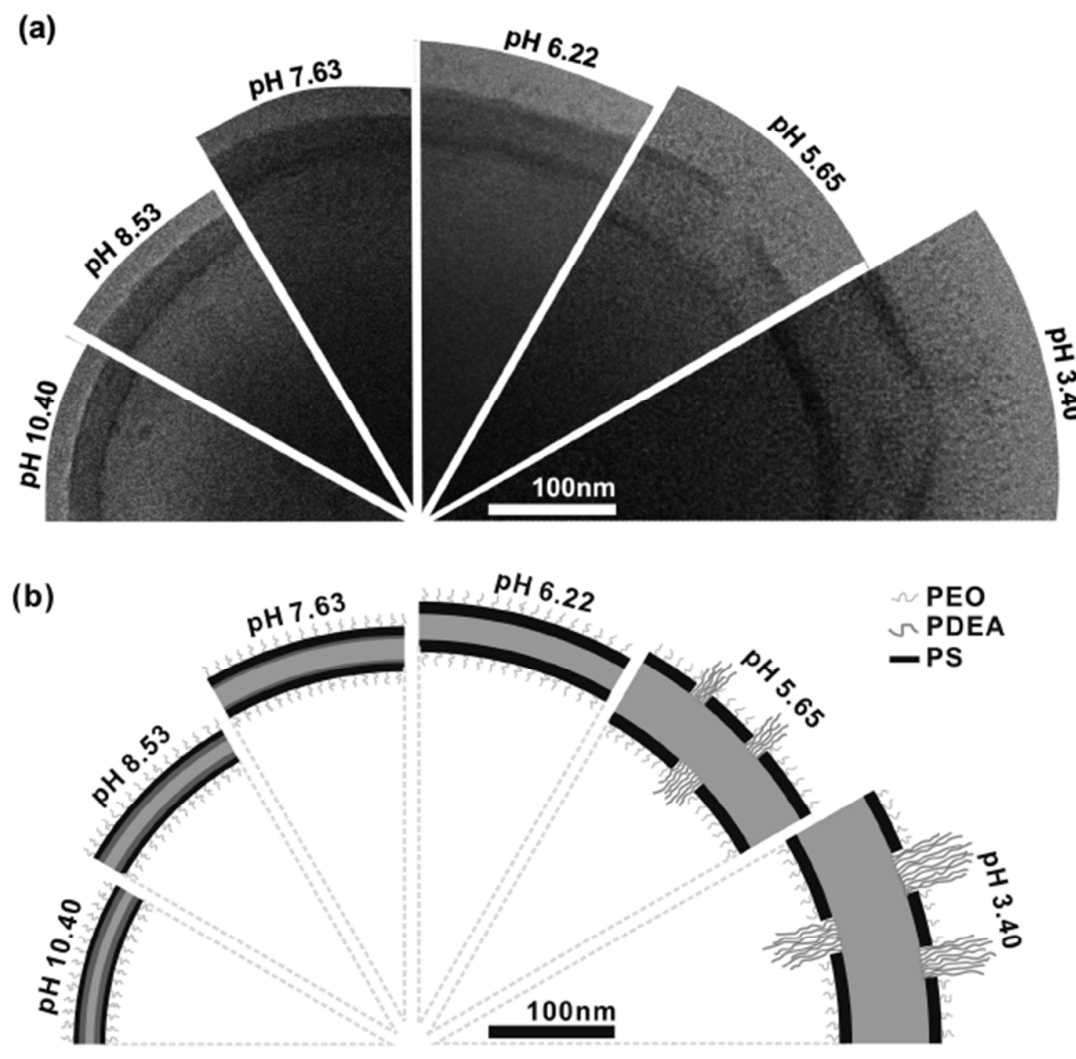

Figure 24. Breathing polymersomes of an ABC triblock copolymer (PEG-PS-PDEA). The membrane thickens (swells) upon acidification, but the strong interactions in the 
polystyrene part keeps the vesicles intact. ${ }^{184}$ (Reproduced with permission from reference 184. Copyright (2009) American Chemical Society).

Studies including a block-copolymer of PS and PDEA as hydrophobic components have proven this concept. ${ }^{184}$ Initially the reversible swelling of this physical crosslinking was reached by adding acid and base, respectively, but was then shown to work also by adding $\mathrm{CO}_{2}$ and $\mathrm{N}_{2}$ to reach the desired $\mathrm{pH}$ switch. In the cross-linking examples discussed so far, PEG was the hydrophilic component, but using PNIPAM a similar result was achieved. ${ }^{174}$ With PCL as the hydrophobic part (and PDMA as $\mathrm{pH}$ sensitive part), the reversible switching is quite remarkable because PCL has lower van der Waals interactions than polystyrene. This makes it unexpected that the polymersomes stay in shape upon the $\mathrm{pH}$ change and do not show a transition to micelles. Again the $\mathrm{pH}$ switch for PNIPAM- $b$-PCL-b-PDMA was achieved with the consecutive addition of $\mathrm{CO}_{2}$ and $\mathrm{N}_{2}$ and the system was claimed to be $\mathrm{CO}_{2}$ responsive rather than $\mathrm{pH}$ responsive. Until now, however, no nanoreactors have been reported using physical cross-linked systems. ${ }^{100,174}$ However, as soon as a block-copolymer with a statistical mix of 2 monomers in the hydrophobic block is used (PS and PDEA), the physical interactions exerted by PS become too weak to hold the polymersome together. With ongoing protonation of a PEG-b-PDEA-r-PS block copolymer and consecutive lack of stabilisation of the residual hydrophobic part, the polymersomes become instable. Protonation makes the polymer hydrophilic and shifts the 
hydrophilic-to-hydrophobic block length ratio. The self-assembly structures change in accordance with previously published results from vesicles to worms and micelles (Figure 25). ${ }^{190}$ The very same principle has also been applied to block-copolymers containing guanidine and amidine derivatives as $\mathrm{pH}$ sensitive part. ${ }^{191-192}$ Literature discusses them as $\mathrm{CO}_{2}$-sensitive, but as the addition of $\mathrm{CO}_{2}$ merely changes the $\mathrm{pH}$, these polymers are discussed as $\mathrm{pH}$ sensitive in this review. What does make these systems special, is that the positive charge it is now stabilised over several bonds by mesomeric resonance structures. It is reasonable to assume that a classic $\mathrm{pH}$ switch would result in the same behaviour. ${ }^{191-192}$ These vesicles have also been deployed to induce a $\mathrm{pH}$-induced switch in morphology, from polymersomes to worms and micelles, depending on the block-length ratio used. ${ }^{191-192}$

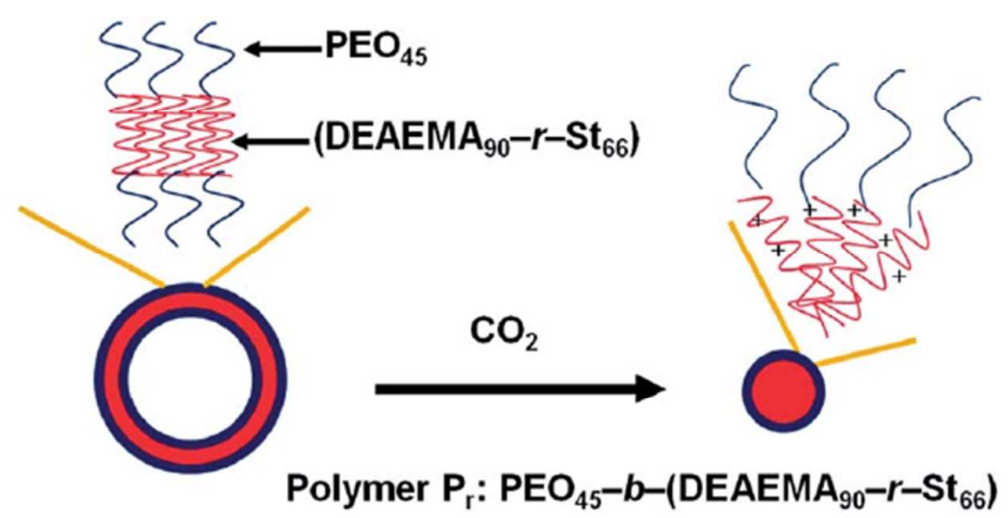

Figure 25. Vesicle-to-micelle transition of a PEG-PDEA-PS with PDEA and PS being incorporated in a random block copolymer. Acidification disturbs the hydrophilic-to-hydrophobic balance, forcing a switch in the self-assembly structures. ${ }^{190}$ 
(Reproduced with permission from reference 190. Copyright (2009) Royal Society of Chemistry).

Apart from the discussed addition of acid or $\mathrm{CO}_{2}$ to change to $\mathrm{pH}$, very recently it was reported by Gu et al., that the vesicles themselves are also able induce a $\mathrm{pH}$ change. A reaction cascade of enclosed glucose oxidase and catalase induces a pH drop as soon as the substrate, glucose, is added to the solution. The ketal units in the hydrophobic block now get hydrolysed in acidic conditions, forcing vesicle disassembly. ${ }^{193}$

\subsubsection{Temperature Responsive Vesicles}

The best known example for a temperature sensitive polymer is PNIPAM with its LCST around $33^{\circ} \mathrm{C}$ and it is, of course, also used for temperature responsive polymersomes. ${ }^{72}$ A LCST behaviour means that the polymer is water soluble below the LCST (hence feasible as hydrophilic component) and water insoluble above it (then suitable as hydrophobic component). One of the first example is thus logically a PEG-PNIPAM block-copolymer where the vesicles are only stable above the LCST of PNIPAM where it is hydrophobic. ${ }^{73}$ Later applications including PNIPAM use it in combination with other functional monomers. Cross-linking was the first property to be combined with PNIPAM. The authors produced a PCEMA-PNIPAM diblock-copolymer which does then form vesicles. After cross-linking, cooling the PNIPAM down did not lead to a destruction of the vesicles, but only to a swelling as already discussed of $\mathrm{pH}$ 
sensitive vesicles. The model compound 4-aminopyridine was released from swollen vesicles at low temperatures only. ${ }^{194}$ A similar approach was used later on using PEG-PAA-PNIPAM polymers where the PAA part was then enzymatically cross-linked. Interestingly, the use of different buffers influenced the LCST. When conducting the experiment in PBS, a LCST of $38^{\circ} \mathrm{C}$ was observed, while experiments in MES buffer gave a LCST of $32^{\circ} \mathrm{C}$. The authors showed that vesicles from this polymer show cellular uptake, no toxicity and a variety of drugs were released successfully. ${ }^{195}$

A rather unexpected polymer to study a temperature sensitive behaviour is PEG itself, especially as it is a standard hydrophilic part for polymersomes. It was shown that the PEG shell is temperature sensitive, although not in a physiological range. ${ }^{196}$ When heated to $75^{\circ} \mathrm{C}$ the hydrophilic PEG chains collapse and cannot support the membrane any longer. Once collapsed, the vesicles turned to dense nano-objects where the hydrophilic and hydrophobic segments form small patches within one another. The collapsing process also means that vesicles of about $200-300 \mathrm{~nm}$ turn into objects of about $100 \mathrm{~nm} .^{196}$

Tertiary amines are also temperature sensitive, which is mainly due to the pKa of the polymers being dependent on temperature. This dependency was shown recently for the PMPC-PDPA polymer where the pKa at $4^{\circ} \mathrm{C}$ is at 7.5 but goes down to 5.6 at $50^{\circ} \mathrm{C}$. No dependency on the chain length of the temperature sensitive part (PDPA) was found by the authors. ${ }^{13}$ The methyl-counterpart PDMA, on the other hand, has been integrated into 
polymersomes to exploit the temperature sensitivity around $35-40^{\circ} \mathrm{C} .{ }^{197}$

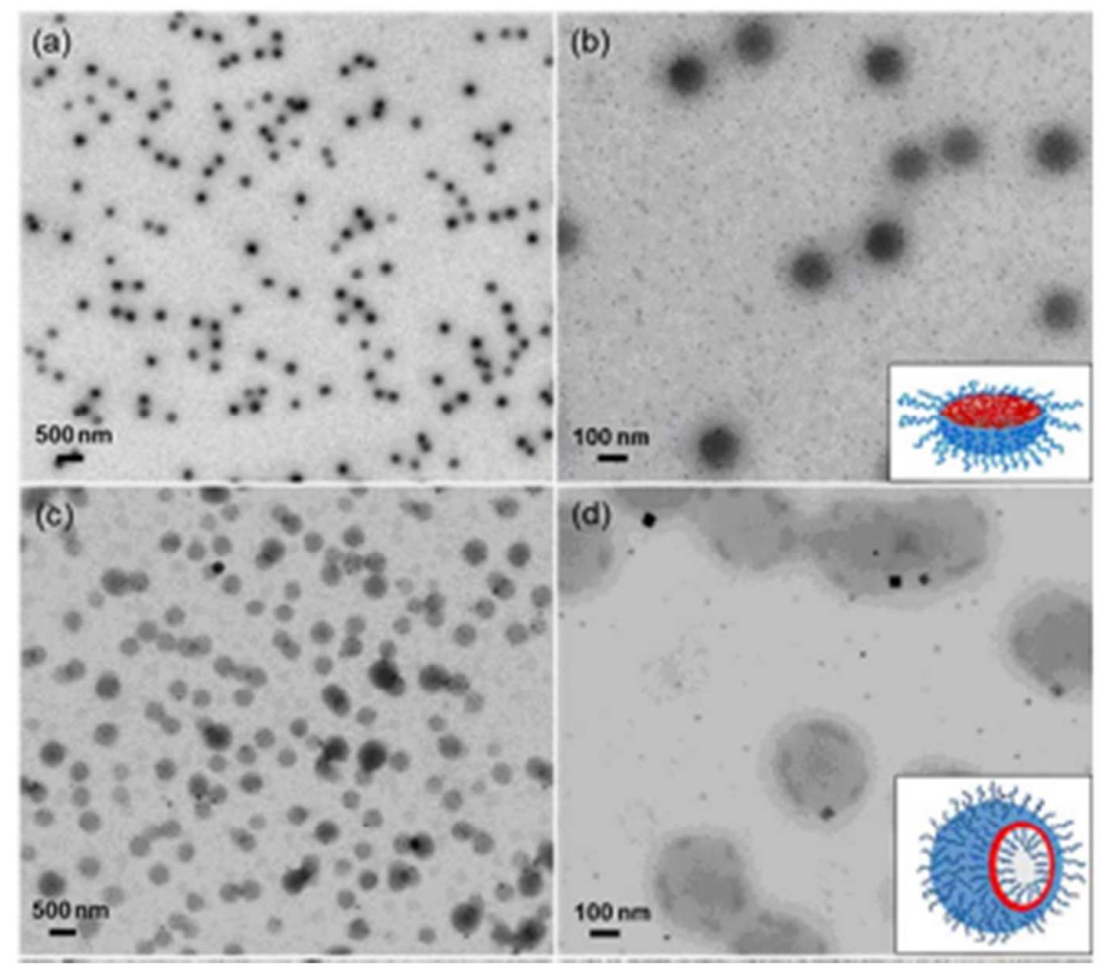

Figure 26. A typical temperature responsive system (here PVCL- $b-\mathrm{PVPON}$ ). Upon heating the polymer assembles into micelles $(a)$ and b): 155-164 resp. block length of PVPON) or vesicles (c and d: 155-404 resp. block length). ${ }^{198}$ (Reproduced with permission from reference 198. Copyright (2014) John Wiley and Sons).

Other, less common cyclic or linear amides also show an LCST behaviour and this was also applied in the production of responsive vesicles. PVCL-PVP samples of various block-lengths are reported by Kharlampieva et al. which can form micelles and polymersomes amongst others. However, they only form above $45^{\circ} \mathrm{C}$ when the PVCL part is hydrophobic. The actual LCST of $\mathrm{PVCL}$ is around $35^{\circ} \mathrm{C}$, but when conjugated with PVP, the observed cloud point rises to $40-45^{\circ} \mathrm{C}$, because the soluble PVP holds the polymer in solution at 
higher temperatures. Stable self-assembled structures are reported for $50^{\circ} \mathrm{C}$ for a variety of different block lengths tested. TEM confirmation for vesicles was obtained for a PVCL $155-b-\mathrm{PVPON}_{164}$ polymer (Figure 26). ${ }^{198}$ Another temperature sensitive amide is trans-N-(2-ethoxy-1,3-dioxan-5-yl) acrylamide (tNEA). Similar to PNIPAM, the polymer is totally dehydrated, e.g. hydrophobic at $37^{\circ} \mathrm{C}$. It is reported as a hydrophobic block in polymersomes where a temperature drop can thus be used to trigger polymersome disassembly and drug release. ${ }^{199}$ A good example for linear amide is aspartamide, and polymers of it with an LCST behaviour were used in vesicles. ${ }^{200-201}$ Especially poly( $\alpha, \beta-N$-substituted-DL-aspartamide)s are reported to show a wide range of possible LCST values, ranging from $20^{\circ} \mathrm{C}$ up to $50^{\circ} \mathrm{C}$. Although the polymer structure does not change (block-copolymer of substituted and unsubstituted aspartamides), the LCST can be adjusted precisely by changing the salt concentration and the $\mathrm{pH}$ of the external solution. ${ }^{200}$

It comes unexpectedly that the hydrophobic poly(benzyl-L-glutamate) (PBLG) in conjugation with PEG or polylysine, shows a temperature sensitive behaviour as well. In recent work, it was shown that PBLG containing polymers show a temperature dependent release profile of an enclosed drug (e.g. doxorubicin). In contrast to previous examples, the membrane becomes more permeable when heated for this polymer. ${ }^{202-203}$

\subsubsection{Light Responsive Polymersomes}

68 
Light is non-invasive and can be applied in a locally confined area, making light-responsive materials very attractive all across science. When it comes to vesicles, two main classes of light-responsive materials are known to literature. The first class uses light to cross-link the membrane, while the second one uses it to change the physical properties of the polymer chains.

The photo cross-linked systems known are combined with $\mathrm{pH}$ or temperature sensitive materials and have been discussed at the corresponding sections with respect to their additional functionality. Here, more emphasis is laid on the cross-linking part (Figure 27). Cross-linking agents reported are based on cinnamoylic acid, ${ }^{204}$ dimethylmaleimide ${ }^{66,186-187,205-206}$ or benzophenone. ${ }^{76,189}$ The first two systems rely on a [2+2] cycloaddition which is only allowed photo chemically but not thermally. ${ }^{207-208}$ The resulting structure is thus defined and the cross-linking moiety remains non-reactive during the polymerisation. The downside of this process is that it requires 2 similar reactive units to form the cross-linking bond hampering the efficiency of the process. Using benzophenone yields radical crosslinking, meaning a less controlled process. However, since the radical can react with almost any part of a neighbouring polymer chain and does not rely on meeting the same unit, cross-linking is more efficient. All of these light-based cross-linking methods are more efficient and yield more stable polymersomes than other examples of cross-linking achieved by hydrolysis of siloxanes ${ }^{209}$ or the addition of an external agent (e.g. diamine, Figure 27). ${ }^{210}$ 

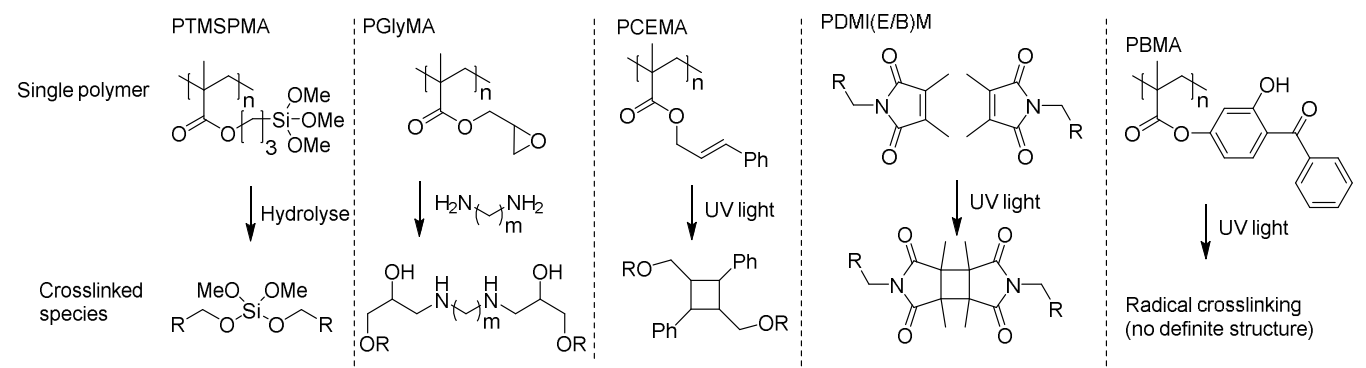

Figure 27. Cross-Linking reactions used in literature exploiting hydrolysis, ${ }^{209}$ external reagents, ${ }^{210}$ UV light for controlled [2+2] cycloadditions ${ }^{66,186,194}$ and radical cross-linking. ${ }^{75,185}$

Independent of the cross-linker used, the amount of cross-linking (e.g. cross-linking density) was shown to affect the final properties of the polymersome. Cross-linking density is known to regulate drug-release, ${ }^{75}$ cellular toxicity, ${ }^{205}$ degree of swelling ${ }^{75,205}$ as well as the stiffness of the actual membrane. 206

The second type of photoresponsive polymers change the physical properties of the membrane without cross-linking it. One class is based on azobenzene derived compounds which change conformation from trans to cis upon irradiation. Polymersomes containing azobenzene derived compounds in the side-chain of the hydrophobic part (azobenzene-modified methacrylate)can be achieved with the functional polymer as part of a linear methacrylate-based polymer as reported by Pei et al., ${ }^{101}$ as part of the main chain as recently shown by Huang et al., ${ }^{211}$ or also as star-like structures as reported by Moretto et al. ${ }^{212}$ and Oriol et al.. ${ }^{213-214}$ The latter ones combine a diazo-containing hydrophobic methacrylate with a PEG-based star polymer 
$\left(P E G_{12}\right)_{3}$ in the hydrophilic part. ${ }^{210}$ The resulting structure is thus a photo responsive miktoarm star polymer. The structural change in the hydrophobic part leads to a disorder in the membrane which can no longer supports its original shape. As a consequence of this conformational stress, drugs start to be able to pass the membrane after irradiation. Moretto et al. use the same principle on a polyamide-based polymer. ${ }^{212}$ Both systems thus allow for a light-triggered drug release (Figure 28). ${ }^{212,210}$ The same holds true for vesicles of a polymer with the reverse architecture of a single PEG-chain connected a dendron containing azo functionality. Irradiation of the sample also leads to membrane distortion and drug release. ${ }^{209}$ The conformational change within diazo polymers can also be used to change the stability of the supramolecular pillar[6]arene-diazobenzene complex. Huang et al. equipped this complex with hydrophilic and hydrophobic units to trigger self-assembly in water. ${ }^{215}$ Upon irradiation and the consecutive trans-cis change, the complex becomes instable and the vesicles disassemble. 

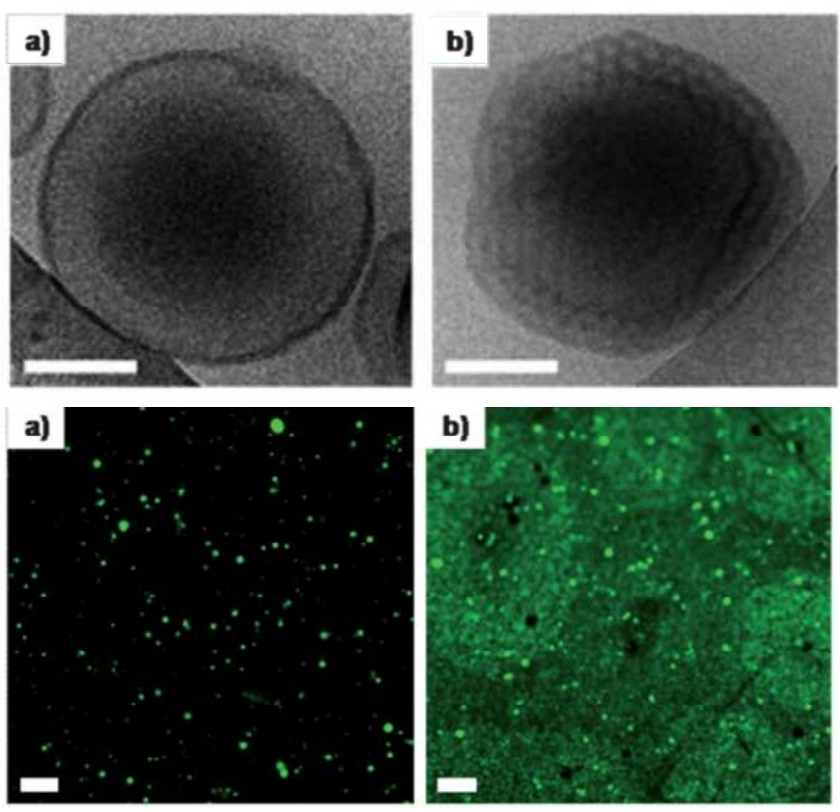

Figure 28. Diazobenzene-based block-copolymer PEG-b-d16isoAZOb for light triggered release. The conformational change distorts the membrane (a to b), making it open for transmembrane diffusion of an encapsulated drug. ${ }^{214}$ (Reproduced with permission from reference 214. Copyright (2013) Royal Society of Chemistry).

Besides inducing conformation changes, light is also known to trigger the degradation of some polymers, for example ones based on nitrobenzene. When it comes to drug delivery, light can be used for a localised drug release with such systems. Meier et al. incorporated a photo cleavable linker between PAA and poly(methylcaprolactone) (PmCL). Cleaving the chains leads to separated PAA and PmCL segments where the latter ones aggregate into small $20 \mathrm{~nm}$-sized particles (Figure 29). ${ }^{216}$ Drugs were not incorporated so far into this system, leaving its potential for biological applications uncertain. Dong et al. also report the use of ortho-nitrobenzyl units, but incorporated them into 
the side chain of their PEG-polyamide block-copolymer. Irradiation leads to the side chains being cleaved off, thus reducing the molecular weight of the hydrophobic block and forcing a switch towards either small vesicles or micelles, depending on the polymer used. ${ }^{217}$
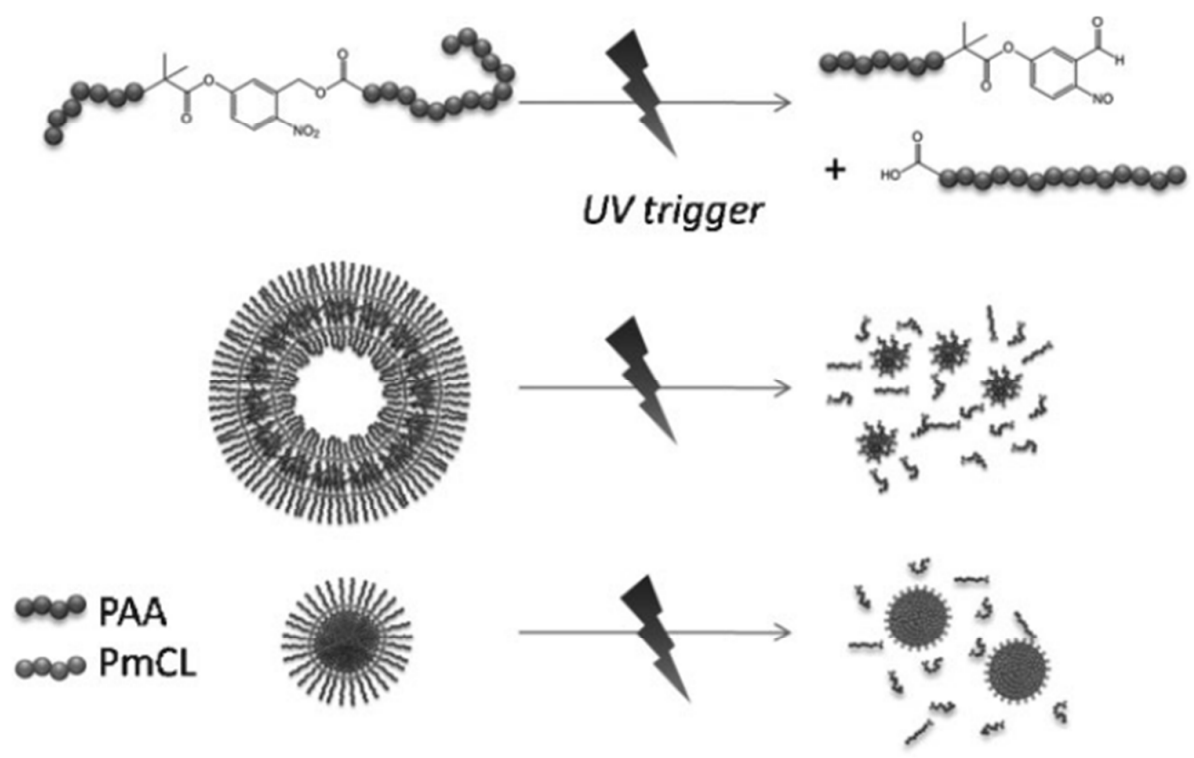

Figure 29. Nitrobenzene units linking $\mathrm{PAA}$ and $\mathrm{PmCL}$ get cleaved upon radiation; the resulting shift in hydrophilic-to-hydrophobic balance forces vesicle disassembly and micelle formation. ${ }^{216}$ (Reproduced with permission from reference 216. Copyright (2010) John Wiley and Sons).

\subsubsection{Reduction and Oxidation Sensitive Polymersomes}

Sulphur containing polymers are readily known to be reduction or oxidation sensitive, depending on their structure. Disulphide bonds can be reduced to two thiols, ${ }^{61,185,218-219}$ or oxidized vice versa, ${ }^{217}$ and a single sulphur atom can be oxidised towards a sulfoxide. ${ }^{217,220}$ Both possibilities are reported for their 
use in polymersomes for triggered drug release and will be discussed in the following section.

In the majority of the reported examples, the disulphide bond is located at the border between the hydrophilic and the hydrophobic part of the polymer with the hydrophilic part being PEG in all cases. A commercially available or custom-synthesised PEG-SH is then conjugated to an initiator or a complete polymer. This can be butyl methacrylate or a cholesterol-modified methacrylate ${ }^{215}$ as examples for radical polymerisation with cholesterol giving the advantage of the additional functionality. With ring-opening polymerisation, poly(propylene glycol)(PPG), ${ }^{61} \mathrm{PCL}^{185}$ and poly(propylene sulphide) (PPS) ${ }^{218}$ have been prepared. Apart from PPG these polymers also carry additional functionality with PCL being biodegradable and PPS can be oxidised, although this was not discussed by the authors in this instance. The perhaps most sophisticated approach in this respect is known from Zhao et al., who created an $\mathrm{AB}_{2} \mathrm{C}_{2}$ miktoarm star polymer with $\mathrm{A}$ being $\mathrm{PEG}, \mathrm{B}$ being $\mathrm{PCL}$ and the $\mathrm{C}$ polymers are either PNIPAM or PDEA to introduce an additional functionality by radical polymerisation. The C-polymers are attached via a disulphide bond to the core moiety. As one can see from the papers mentioned, the reduction sensitivity is usually combined with another functionality. This ranges from biodegradability to sensitivity towards $\mathrm{pH}$ or temperature, amongst others. All of these papers report a complete cleavage of the disulphide bonds within 10 minutes of incubation either into cells or treatment with DTT. The PEG corona 
is chopped off, leaving a plain hydrophobic polymer which does no longer support vesicles leading to complete drug release (Figure 30). Biodegradable polymers like PCL are of course useful, as the residual chunks can now be digested safely in vivo. ${ }^{221-222}$ The miktoarm star, however, does not disassemble directly, but the lower amount of hydrophobic polymer forces a conformational change from vesicles to micelles. But also here the addition of DTT leads to a significant increase in drug release.

In a prominent exception, the disulphide bond is located within the PEG itself. Once cleaved, the hydrophilic part of the amphiphilic block-copolymer gets shorted, hence disturbing the hydrophilic-to-hydrophobic balance. The authors use this shift to promote a change from micelles into polymersomes upon disulphide cleavage. ${ }^{81}$
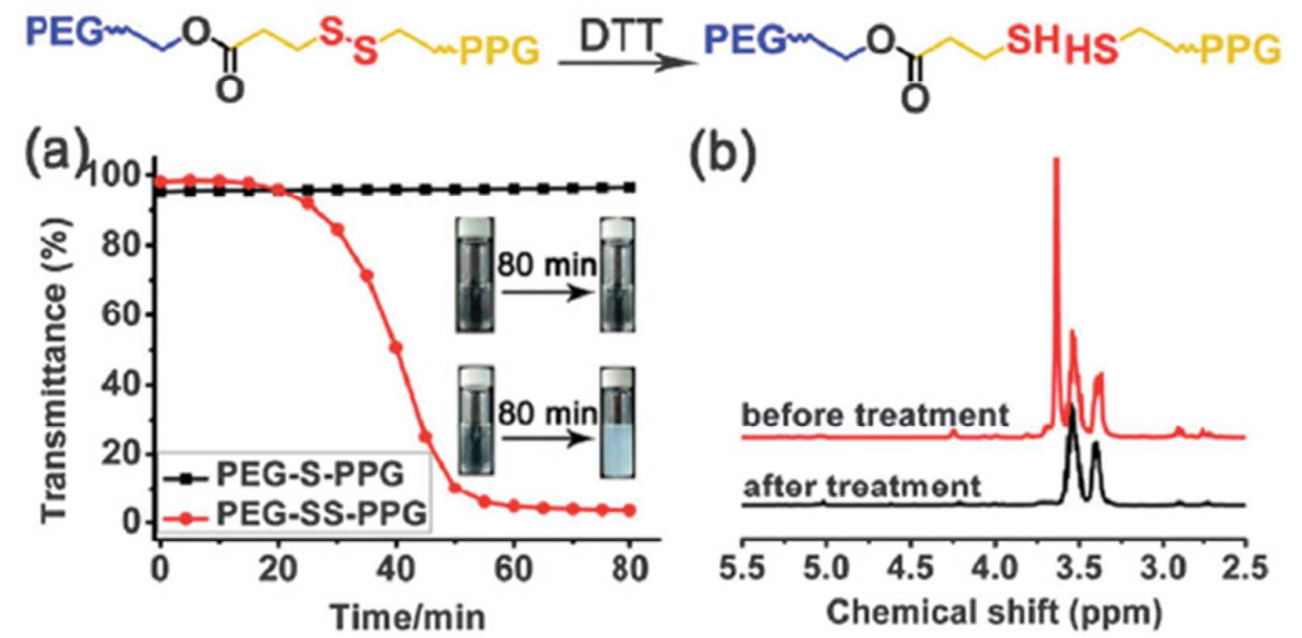

(b)

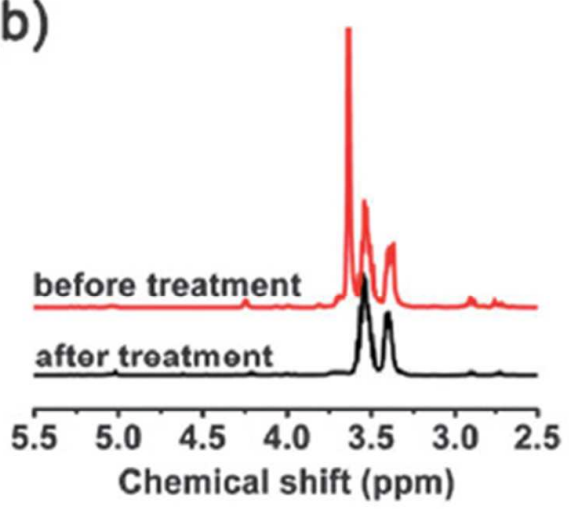

Figure 30. A typical disulphide-containing sensitive polymersome: the hydrophilic part (here PEG) is linked to the hydrophobic part (here PPG) via a disulphide bond, which can be cleaved using DTT or enzymes, leading to a destruction of the vesicles. A disulphide 
bond is necessary for vesicles destruction as opposed to a thioether (a). Bond cleavage also leads to a distinctive change in the NMR spectrum (b). ${ }^{61}$ (Reproduced with permission from reference 61.Copyright (2014) Royal Society of Chemistry).

Besides reduction of a disulphide bond, oxidation of thiols to create disulphides is also an option (Figure 31). ${ }^{220}$ To achieve this in a controlled fashion the thiols need to be protected beforehand to prevent autooxidation. Dong et al. reported polypeptides with o-nitrophenyl units which could be cleaved photo chemically as discussed in the corresponding section. ${ }^{217}$ The resulting thiols were then used to crosslink the residual polymeric micelles by adding DTT and dilute $\mathrm{H}_{2} \mathrm{O}_{2}$. Besides crosslinking the polymersome membrane, some inter-micelle cross-linking was also reported by the authors, but not to the extent of resulting in precipitating particles. Reversing the steps was also attempted by the authors, e.g. first oxidising the sulphur. In this case, a sulfone was created ultimately decreasing the hydrophobicity of the polymer. Although it did not destroy the polymersomes, it did result in shrinkage of them. ${ }^{217}$ Hubbell et al. pioneered the work of oxidising polymersomes (Figure 31). ${ }^{220}$ In their simple PEG-PPS-PEG system, stable vesicles were formed initially and ultimately destroyed by oxidising the sulphur atoms of the PPS to sulfoxides and further to sulfones. Both versions are far more hydrophilic than the initial PPS, leading to a complete disassembly of the vesicles towards single polymer chains. ${ }^{220}$

Alike $\mathrm{pH}$ sensitive vesicles, ones from oxidation/reduction block-copolymers 
can of course also be crosslinked. Yoshida et al. achieved this by incorporating a Ruthenium initiator for controlled radical polymerisation and thus the ability to crosslink methacrylamide units within the polymer. Once this is achieved, the polymersomes show a swelling-deswelling behaviour similar to cross-linked $\mathrm{pH}$ sensitive vesicles, although less reproducible in terms of size. ${ }^{223}$

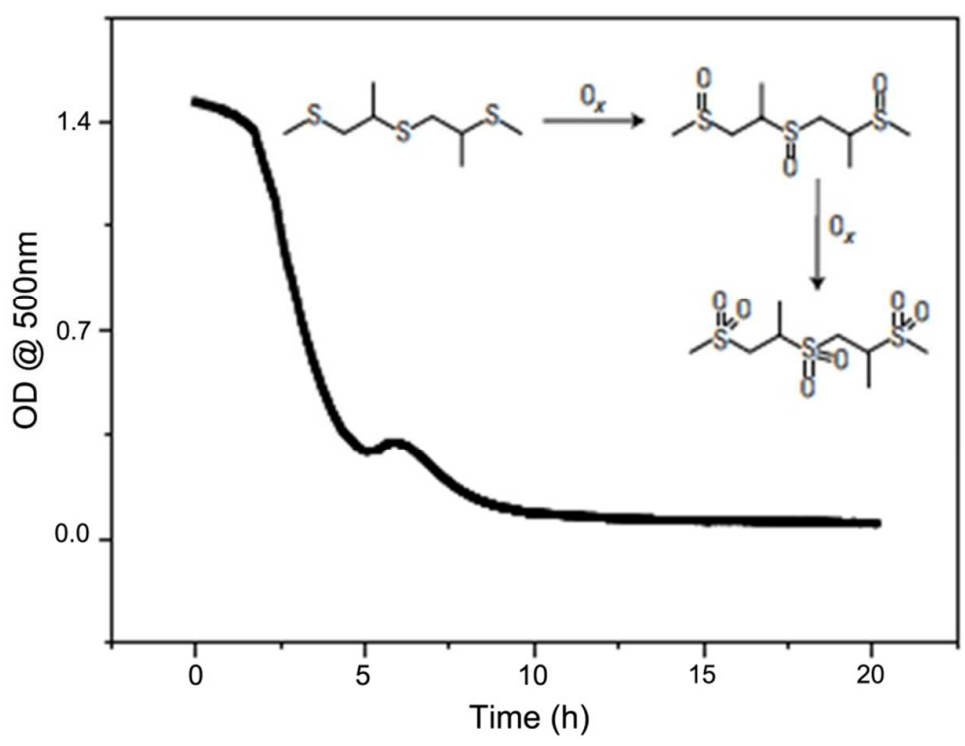

Figure 31. Rapid increase in transmission upon oxidation of the PEG-PPS block-copolymer due to oxidation of the sulphur atoms. This leads to a disassembly of the polymersomes. ${ }^{220}$ (Reproduced with permission from reference 220. Copyright (2004) Nature Publishing Group).

Replacing existing thiols by new ones merely by stoichiometric excess of the new ones, can also induce a vesicle response if the new thiol has distinctively different properties than the old one. O'Reilly et al. exploit this to force a conformation change from micelles to vesicles purely by thiol exchange. ${ }^{224}$ The new thiol is of aromatic nature and more hydrophobic than the old one 
with an ethanol residue. This pushes the hydrophilic to hydrophobic ratio towards the hydrophobic fraction, forcing the change.

\subsubsection{Solvent Responsive Vesicles}

Besides the physical stimuli just discussed, polymersomes can also react to a change in the solvent surrounding them. The solvent polarity must of course not fall below the threshold where both polymer blocks are soluble and reverse the solvent-switch method to create polymersomes. But if one has a closer look at this solvent switch method, there is an important issue to be aware of. When water is added slowly to the polymer solution and the vesicles form, there is of course some organic solvent in the mixture, which is then dialysed away. It is this slight switch in solvent polarity to which some polymersomes react with a change in morphology to produce totally collapsed or "kippah" vesicles. The authors chose this nickname in line with previous terms in science which are derived from mythology or religion, such as Janus particles. $^{225}$ The term has since spread to pure silica vesicles ${ }^{226}$ and silica/organic hybrid nanospheres. ${ }^{227}$

The first example of kippah vesicles from Eisenberg et al. describes a $\mathrm{PAA}_{47}-\mathrm{PS}_{434}$ system where vesicles are prepared in multiple variations of the solvent switch method. ${ }^{225}$ The crucial bit is always when the residual amount of organic solvent is removed. If a water-miscible solvent is used, as it has to be for most cases of a solvent switch (see according section), it leaves the 
polymersome through the membrane and is supposedly replaced by pure water to keep the internal volume of the polymersome constant. For polystyrene, however, the water cannot diffuse back because the membrane is too glassy and hydrophobic to allow water to travel through it. The internal volume of the polymersome shrinks in that consequence and the membrane bends in - as proposed by the authors. ${ }^{225}$ Further, more extensive studies by van Hest et al. use a $\mathrm{PEG}_{45}-\mathrm{PS}_{230}$ system, so also the glassy polystyrene is necessary. In a first publication, the authors are able to confirm the previously purely speculative mechanism proposed above, by showing TEM images at various stages of the process. ${ }^{228}$ This left the question whether the process is reversible to some extent and whether the solvent mixture used has an effect on the process. Reversibility was proven by adding a small amount of organic solvent, which made the membrane just a small bit permeable and allowed it to inflate to give the energetically favoured vesicle structure (Figure 32). ${ }^{65}$ This opens the question why this does not happen beforehand when the kippah vesicles form, as a small amount of organic solvent is left in the solution until the last moment. ${ }^{67,228}$ The answer is that some solvents have plasticising and some deplastisising effects on polystyrene. It was shown that dioxane results in a more rigid PS membrane while THF makes it permeable towards organic solvents and water. ${ }^{65}$ Interesting enough, this leads to the possibility to create kippah vesicles as well as flat, oblate-like structures and normal vesicles just by changing the solvent the polymersomes are dialysed against. ${ }^{65}$ Very 
recently, the authors demonstrated that this transition in shape can be monitored using magnetic birefringence. ${ }^{229}$ or even can be used to control the shape using magnetic fields. ${ }^{230}$
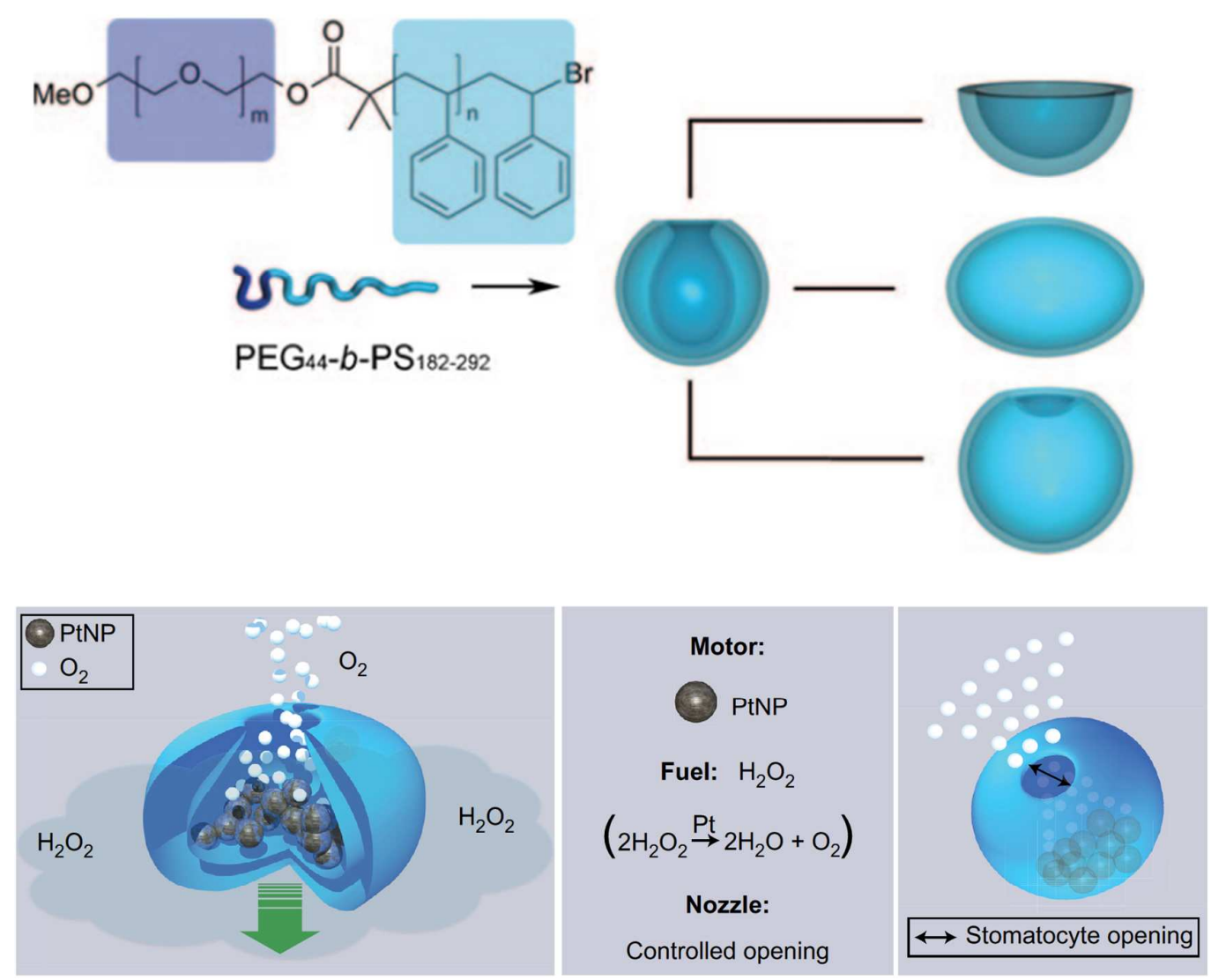

Figure 32. The formation of kippah vesicle (top), oblates and vesicles with indentations from the solvent responsive PEG-PS system. ${ }^{65}$ Once formed, they can host Pt nanoparticles (bottom) which can be used to catalyse the decomposition of hydrogen peroxide to propel the nanoparticle. ${ }^{231}$ (Reproduced with permission from reference 65 and 231. Copyright (2011) John Wiley and Sons and Copyright (2012) Nature Publishing Group, respectively).

Once formed, these collapsed vesicles (also called stomatocytes) can 
perform multiple tasks due to the cavity created. Just like normal polymersomes, stomatocytes can enclose hydrophilic substances in their core. The main difference is that the shell is now open to one side allowing for the diffusion of small molecules while prohibiting the diffusion of larger ones. ${ }^{232}$ It was shown, for example that reactive enzymes as well as metal nanoparticles can be enclosed into the inner cavity (or stomach) of the stomatocytes during their formation process. ${ }^{231-232}$. With respect to nanoparticles, the stomatocytes can even act as template for their growth. ${ }^{232}$ It has been shown that the enclosed Pt nanoparticle can now catalyse a reaction, like the decomposition of $\mathrm{H}_{2} \mathrm{O}_{2}$, and in this case the formed gaseous $\mathrm{O}_{2}$ can propel the vesicle. ${ }^{231}$. As one can expect, the amount of fuel governs the speed of the vesicles. ${ }^{233}$.

It should be noted that the mixing of two immiscible polymers can also lead to changes in the topology of the polymersome surface. Similar indentations as for kippah vesicles are reported for a $P A A_{40}-P D_{110}$ and $P E G_{45}-P D_{110}$ mixtures, also by the van Hest group. ${ }^{234}$ However, such phase separations are also known to work the other way around and result in protrusions on the outside of the vesicle as reported for a great variety of PEG, PEE and PMPC mixtures by Battaglia et al.. ${ }^{235-237}$

\subsubsection{Combined Responsiveness}

Until here, we focussed on the many different ways of how to include sensitivity into vesicles. The current trend is towards multiple responsiveness, 
e.g. to combine responsiveness towards different stimuli into one vesicle. Many of the examples discussed do so, although we only highlighted one of the functionalities until now. If one has a look at statistics, the number of possible combinations of the above mentioned ways of responsiveness leads to a large number of possible copolymers, either as blocks or as random copolymers. The principle of how the then formed vesicles work, however, does not deviate and is always very similar. Upon a first response, the hydrophobic part of the polymersome becomes shorter or is weakened, triggering the transformation into micelles. A second switch then yields the disassembly into unimers. ${ }^{95,96,186}$ Due to this, we will not cover them in detail within this review.

The story changes slightly for the cross-linked polymersomes which are also already covered extensively in the sections above. Their first responsiveness, the one towards light is irreversible, and cross-links the vesicles. The second one, which can be of any nature ( $\mathrm{pH}$, temperature or oxidation) is reversible and causes a reversible swelling of the polymersomes as discussed. Due to their popularity, the combination of temperature and $\mathrm{pH}$ sensitivity into polymersomes is discussed separately. One way to achieve this is to use PDMAEM in the polymer as it is $\mathrm{pH}$ sensitive and temperature sensitive (see above). One can, of course, also use two different polymers to get the dual responsiveness. Typically, PNIPAM is the temperature-sensitive polymer and one of the amine containing polymers serves as the $\mathrm{pH}$ sensitive part. ${ }^{186,238-239}$ 
It should be noted that the amount of responsiveness is of course not limited to two functionalities. Zhao et al. ${ }^{195}$ and Pei et al. ${ }^{101}$ included three functionalities into polymersomes which led to temperature and $\mathrm{pH}$ sensitivity, and in addition, either light responsiveness (diazo bond) ${ }^{101}$ or redox sensitivity (disulphide bond). ${ }^{195}$ This allows to cut a fine balance between controlling the self-assembly state and permeability of the membrane as it has been shown in the corresponding sections of each of the sensitivities.

\subsection{Core-Based Design}

It has been widely accepted that the unique structure of hollow polymer capsules consist of a polymer membrane domain and an empty core domain. To engineer functional polymer capsules towards smart nanoreactors, in addition to the above-mentioned modulation of the polymer membrane, the other significant aspect is the modulation of the empty core domain. Subsequently, in this subsection, we will focus on the discussion about what can be done for modifying the core domain, including the incorporation of another small polymer compartment or inorganic particles into the core.

\subsubsection{Multi-Compartment Model}

Being inspired by biological cells which are able to perform multiple complex reactions within confined environments owing to their internal sub-compartment structure, the development of multi-compartment polymer capsules is now attracting more and more researchers' attention. ${ }^{240-244}$ The 
constructed multi-compartment polymer capsules, to be considered as a new generation of polymer capsules, definitely will bring a new way for the application of the polymer capsules as nanoreactors. The concept of multi-compartment polymer morphology was first raised by Ringsdorf ${ }^{245}$ twenty years ago, and then was widely developed. ${ }^{246-248}$ Up to now, the created multi-compartment polymeric capsules include: vesosome, ${ }^{249}$ a liposome in liposome structure formed by smaller vesicles encapsulated within a bigger vesicle; polymer vesosome ${ }^{85,250}$ a polymersome in a polymersome structure formed by using different polymersomes as the carrier and sub-compartment structures; dendrimersomes formed by the self-assembly of Janus dendrimers in solution with predictable size and number of bilayers; ${ }^{15,251-258}$ capsosomes $^{243,259-260}$ formed by the LbL method to encapsulate sub-compartments in the shell or in the core of a polymeric capsule; and other polymer capsules in polymer capsule models synthesized by self-assembly, ${ }^{261}$ surface-initiated polymerisation, ${ }^{262}$ double emulsion ${ }^{263-264}$ or microfluidic methodology etc. ${ }^{93}$

For example, using a simple method based on sequential self-assembly Nallani and coworkers fabricated larger polymersomes of poly(styrene)-b-poly(L-isocyanoalanine(2-thiophen-3-yl-ethyl) amide) (PS-b-PIAT) with an encapsulated suspension of smaller polymersomes in the core. $^{261}$ While the encapsulation efficiency of the smaller polymersome in the core was relative low, in order to improve this, as a powerful alternative method 
emulsions or double emulsions technique was developed. Using a double emulsion technique in a water/oil/water system, Chiu and coworkers demonstrated to generate a multi-compartment capsule by encapsulating small vesicles within large polymersomes with $\mathrm{pH}$-responsive membranes. ${ }^{263}$ In detail, copolymers were dissolved in an organic phase, followed by emulsification and evaporation of the organic solvent (THF and $\mathrm{CHCl}_{3}$ ) to form polymer vesicles. These preformed vesicles were subsequently introduced into a water phase of the second-stage double-emulsion process to form the multicompartment assembly in size range from 1 to $15 \mu \mathrm{m}$. The size of these vesicles can be tuned by varying the ratio of $\mathrm{THF}$ to $\mathrm{CHCl}_{3}$ and by changing the content of the copolymers. In this system, the outer and inner compartments are composed of poly(acrylic acid)-co-poly(distearin acrylate) $($ poly $(A A c-c o-D S A)) \quad$ polymersomes equipped with $\mathrm{pH}$-responsive transmembrane channels (Figure 33a), allowing the controlled release of encapsulated cargo in response to environmental pH changes. Interestingly, such $\mathrm{pH}$-responsive process is reversible in the range of $\mathrm{pH} 5$ to 8 . Even though these polymersomes are impermeable to small hydrophilic solutes, such as calcein at $\mathrm{pH} 5$ (Figure 33b), a change in the ionisation state of the acrylic acid units at $\mathrm{pH} 8$ could result in the formation of pores and subsequently allows the transport of the encapsulated cargo across the membranes (Figure 33c). ${ }^{263}$ 
a

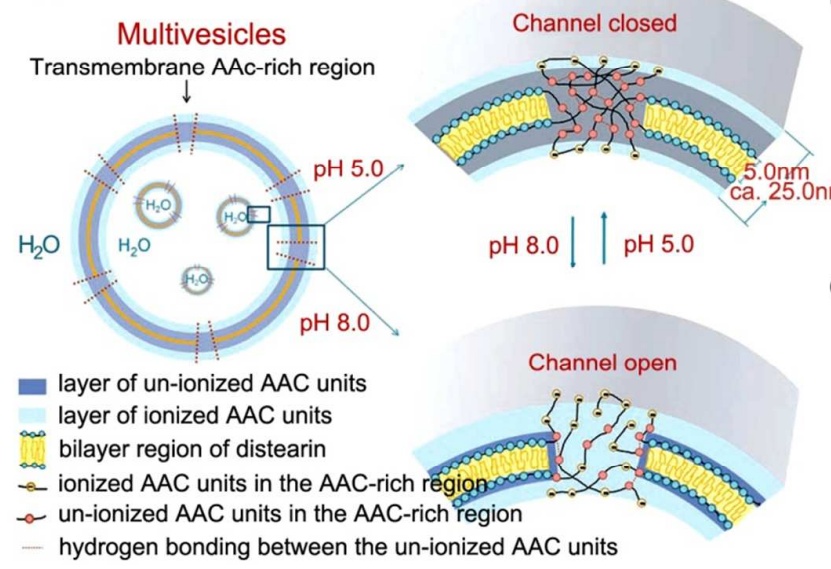

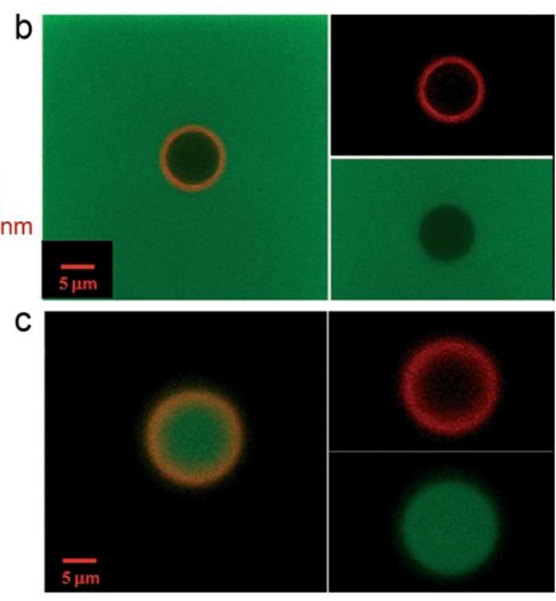

Figure 33. (a) Illustration of multi-compartment assemblies equipped with pH-responsive transmembrane channels from a two-stage double emulsion vesicle formation process using poly(AAc-co-DSA). (b) Confocal laser scanning microscopy images of Nile red-stained polymersomes with the addition of calcein at $\mathrm{pH} 5.0$ (calcein cannot diffuse into polymersomes), and (c) after adjusting the $\mathrm{pH}$ to 8 (calcein diffuses into polymersomes) and replacement with fresh $\mathrm{pH} 5$ buffer (calcein is confined with the polymersomes). ${ }^{263}$ (Reproduced with permission from reference 263. Copyright (2008) John Wiley and Sons).

To develop multi-compartment capsules, such emulsion process was further improved by Weitz and coworkers by combining emulsion with microfluidic technique. ${ }^{265}$ As demonstrated in section 2.1, the main advantage of this technique is the ability to control the number as well as the size of the compartments in the assembled models simply by tuning the flow rates of the phases in the microfluidic channels and changing the capillary diameter, respectively. The size of the multi-compartment polymer capsules could be 
varied ranging from 50 to $200 \mu \mathrm{m}$ in diameter, with containing 2-8 inner compartments. Also the composition of the membrane can be changed freely by using different polymers as building blocks. Namely, both, the external and internal polymer capsules could be composed of biocompatible poly(ethylene glycol)-b-poly(lactic acid) (PEG-b-PLA) diblock copolymers, with the controlled release of the encapsulated cargo induced by the introduction of mechanical strain or selective dissociation of the polymersome membranes by hydrolysis..$^{93,266}$

Very recently, as a further development in this field it was demonstrated by Lecommandoux and co-workers that a polymersome in a polymersome model was generated using a facile, versatile, reproducible, and low-time and product consuming technique based on the emulsion-centrifugation method (please also refer to section 2.1.2). ${ }^{18,85,250}$ The inner polymersomes were formed by nanoprecipitation of poly(trimethylene carbonate)-b-poly(L-glutamicacid) (PTMC-b-PGA). ${ }^{267}$ This suspension was then loaded in larger polymersomes of polybutadiene- $b$-poly(ethylene oxide) (PB- $b$-PEO) by emulsion-centrifugation. ${ }^{268}$

Due to the distinctive structure of multi-compartment polymeric capsules with multiple hollow core domains and a tunable polymer shell, one can easily foresee promising applications in different fields. ${ }^{249,269}$ For example, multiple sub-compartments in a polymeric capsule might be considered as individual nanoreactors able to perform multiple complex reactions within confined 
environments to simulate simple steps of cell activity; capsules that contain multiple batches of drug carriers in compartments of different hierarchies can serve as a depot for highly controlled and sustained release of drugs. In this regards Caruso and coworkers have shown the construction of a serial of multi-compartment polymeric capsules based on the LbL method and using liposomes or small size polymeric capsules as sub-compartments. ${ }^{242,259,270-273}$ These model capsules were applied as nanoreactors and drug carriers by encapsulation of an enzyme, ${ }^{250}$ pDNA $^{274}$ or hydrophobic ${ }^{270}$ and hydrophilic ${ }^{275}$ moieties within the liposomal sub-compartments. It was also possible to perform a two-step enzymatic catalytic reaction, whereby reduction of a substrate is exploited to subsequently trigger cargo release. ${ }^{244}$ As demonstrated in Figure 34, the authors encapsulated glutathione reductase into liposomes, which were then embedded into polymer carrier capsules to form capsosomes. The architecture of the capsosomes enables a temperature triggered conversion of oxidised glutathione to its reduced sulfhydryl form by the encapsulated glutathione reductase. The reduced glutathione subsequently induces the release of the encapsulated oligopeptides from the capsosomes by reducing the disulphide linkages of the conjugates. This study well highlights the potential of capsosomes to continuously generate a potent antioxidant while simultaneously releasing small molecule therapeutics. 


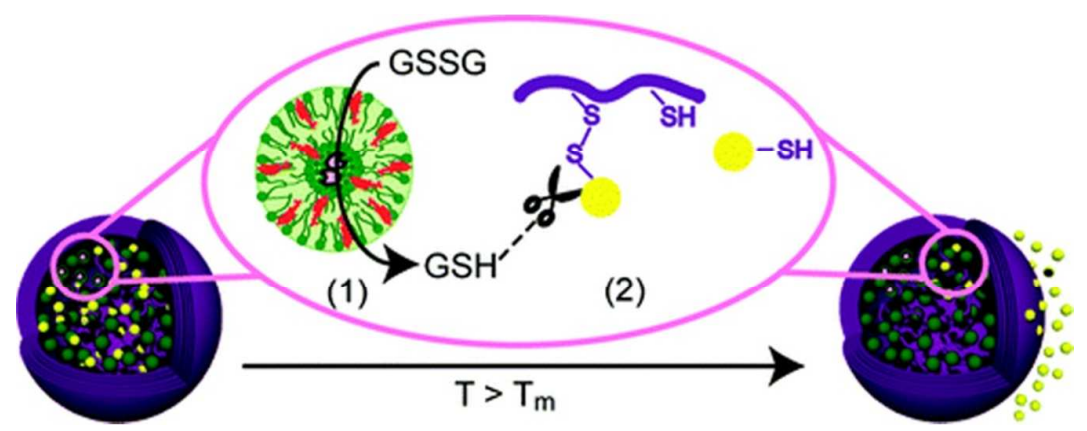

Figure 34. Schematic illustration of a temperature-triggered catalysis of an encapsulated glutathione reductase in the "Free-Floating" liposomal subcompartments of capsosomes. In there glutathione disulphide (GSSG) bonds are reduced to its sulfhydryl form (GSH). Consecutive release of encapsulated oligopeptides was triggered by the catalytic activity of glutathione reductase within the capsosomes. ${ }^{244}$ (Reproduced with permission from reference 244. Copyright (2011) American Chemical Society).

\subsection{2 "Yolk-Shell" Model}

Apart from the incorporation of the sub-compartment structure into the core, to engineer the core domain of polymer capsules, another effective way is to directly encapsulate a functional component in the core, subsequently in the unique hollow polymer structure, a "rattle" or "yolk-shell" morphology is developed. For example, Kang and coworkers synthesized narrowly-dispersed silver@silica@poly(methacrylic acid) (Ag@SiO $\left.{ }_{2} @ P M A A\right)$ core-double shell hybrid nanoparticles via distillation-precipitation polymerisation. ${ }^{276}$ Selective removal of the inorganic silica inner-shell from the $\mathrm{Ag} @ \mathrm{SiO}_{2} @ P M A A$ core-double shell hybrid nanoparticles by HF etching produced the Ag@air@PMAA hybrid nanorattles with a silver nanocore, PMAA shell and 
free space in between. The as-synthesized Ag@air@PMAA hybrid nanorattles were also explored as a nanoreactor system for confined catalytic reduction of p-nitrophenol, of which the rate of catalytic reaction can be further regulated by controlling molecule diffusion in and out of the stimuli-responsive PMAA shell through the simple variation of environmental stimuli, such as salt $(\mathrm{NaCl})$ concentration in the medium. ${ }^{276}$

As another special type of multi-compartment models, onion-like capsules consisting of concentric capsules with uniform spacing between the walls are also attracting more and more attention. The dense onion-like structures were first found in bulk block-copolymer blends over twenty years ago. ${ }^{277}$ Later such onion-like structures had been formed in solution and various building blocks were employed ranging from small molecule surfactant systems ${ }^{278}$ to block copolymers $^{279-280}$ and to very recently reported Janus dendrimers. ${ }^{253}$ For example, onion-like dendrimersomes reported by Percec et al. were obtained via self-assembly by simple injection of a solution of Janus dendrimers into a water-miscible solvent, into water or into buffer. ${ }^{253}$ Significantly, the size and the number of bilayers of the onion-like capsules were predictable by the final concentration of the Janus dendrimers. At low concentration, double-bilayer capsules were formed, whereas at higher concentrations they formed multibilayer onion-like capsules. Undoubtedly, such onion-like dendrimersomes provide simple and easily accessible mimics of various double-bilayer and multibilayer biological membranes of different cells and 
organelles, such as Gram-negative bacteria or multilamellar bodies etc.

\section{Application of the Polymer Capsule Nanoreactors}

A typical polymer capsule as described above has an aqueous lumen sequestered from the external aqueous environment by a polymer membrane and therefore can encapsulate both hydrophilic and hydrophobic cargo either into the membrane domain or the core domain. As has been discussed above, a wide range of techniques can be applied to generate a diversity of stable and robust polymer capsules including polymersomes, polymeric capsules or proteinosome etc. ${ }^{14,38,40,281-282}$ The unique structure of polymer capsules is of special interest and is attracting more and more researchers' attention, in view of their promising application as nanoreactors. Generally, to exploit polymer capsules as nanoscale reaction vessels, two main requirements have to be considered. ${ }^{10}$ One is that catalytically active guests need to be encapsulated within their inner compartments to confine the reaction into the polymer capsules, and the other one is that the semipermeable polymer membrane, which separates the inner aqueous compartment from the outer bulk solution, should allow the exchange of substrates between the outside and the inside of polymer capsules. ${ }^{10,18,30,283}$ Here, the former requirement can be easily met, since the strong encapsulating ability is one of the characteristic features of polymer capsules, especially for the ones which were synthesized by self-assembly or miniemulsion method. For example, the reported 
proteinosome model showed that a GFP cell-free gene expression system containing a hundred or so components could be integrally encapsulated into the core without affecting the GFP expression within the proteinosome internal volume. ${ }^{16}$ For the latter requirement, in comparison to liposomes, polymer capsule membranes are often several times thicker because of the higher molecular weights of copolymers compared to those of typical phospholipids which makes the membrane, especially for the polymersome system, even less permeable to small water-soluble molecules. However, with the advances made in polymer chemistry in recent years, it is becoming much easier to tune the permeability of the membrane as well as their surface functionality by both chemical and physical strategies. By the chemical design and synthesis of various smart copolymers as building block (see section 3.1 ), the permeability of the polymer capsules can be well modulated by responding to external stimuli including $\mathrm{pH}$, light, redox and solvent etc. ${ }^{47,132,284-288}$ Alternatively, as demonstrated very recently, tuning the permeability of the membrane was also studied by some physical strategies through incorporating channel proteins within membranes, choosing different length of bifunctional compound as cross-linkers or controlling the shear-rate etc. ${ }^{31,111,186,277}$ Taken together, it is no doubt that these well-developed strategies provide an effective way to construct different functional nanoreactors. In the following section, the discussion will be given based on the application of the polymer capsule nanoreactors in enzyme catalysis, polymerisation, nanoparticles synthesis and 
as artificial organelles etc.

\subsection{Application of the Polymer Capsule Nanoreactors for Enzyme Catalysis}

For the application of polymer capsules as nanoreactors, a straightforward way is to encapsulate active guests within the inner compartment of polymer capsules. ${ }^{10,29,35}$ For example, a very recent study showed that an enzymatic nanoreactor was constructed by incorporating $\beta$-galactosidase into a polyion complex vesicles (PICsomes), as shown in Figure $35 .{ }^{27}$ In this system, by tuning the hydrophobicity of the aliphatic side chains in the pendant group of the constituent polyelectrolytes, the non-cross-linked PICsomes demonstrated sufficient stability at physiological salinity and temperature. Even in the presence of trypsin in the exterior, the activity of the encapsulated $\beta$-galactosidase was well maintained. ${ }^{27}$ Up to now, a host of polymer capsule-based nanoreactors was constructed by encapsulating peroxidases, ${ }^{47,289-290}$ penicillin acylase ${ }^{291}$ superoxide dismutase ${ }^{32,283,284,158}$ catalase $^{32,158,292-293}$ myoglobin, ${ }^{111,174,186,294}$ galactosidase, ${ }^{27}$ lysozyme, ${ }^{285}$ lipase, ${ }^{33,86}$ phenylacetone monooxygenase, ${ }^{81}$ alcalase, ${ }^{81}$ alcohol dehydrogenase ${ }^{81,295}$ laccase ${ }^{296}$ or alkaline phosphatase,${ }^{297}$ respectively, in the core. Within the confined space provided by the polymer capsule, reactions can occur with higher selectivity or less side reactions. ${ }^{298}$ Furthermore, it has been shown that reactions and proteins could be monitored on the single molecule level using nanoreactors. ${ }^{299-300}$ Cascade reactions of two or more 
catalytic species become more efficient due to the close spatial proximity and precise positioning of the catalysts. ${ }^{166,301-302}$ Considering that most enzyme-loaded nanoreactors need to be able to perform enzymatic reactions in situ with membrane diffusion of substrates and products, the above-mentioned successful tuned permeabilisation strategies (in section 3.1) will enable a controlled enzyme-mediated catalytic behaviour for the constructed nanoreactor. ${ }^{107,182}$ For example, Voit and coworkers loaded myoglobin into a $\mathrm{pH}$ sensitive poly(diethylaminoethylmethacrylate) (PDEAEM) capsules $^{107}$ (see also Figure 23). ${ }^{187}$ As anticipated, to be a nanoreactor, its catalytic efficiency can be well modulated by $\mathrm{pH}$. Since at high $\mathrm{pH}$ value $\mathrm{pH}$ 8.0), the PDEAEM membrane is deprotonated and fully hydrophobic, the hydrophilic substrate hydrogen peroxide cannot easily diffuse through the hydrophobic membrane to reach the encapsulated myoglobin in the core, thus showed a relative low catalytic activity. While, after adjusting the $\mathrm{pH}$ value of the measured solution to $\mathrm{pH} 6.0$, an obviously enhanced activity was observed. This is due to the fact that the nanocapsule membrane is now in a swollen state and totally hydrophilic which makes the transmembrane diffusion possible, thereby allowing the substrates both guaiacol and hydrogen peroxide to reach the myoglobin in the core and accelerate the reaction. 

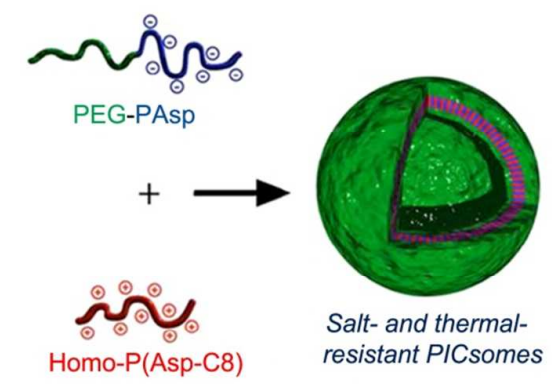

Salt- and thermalresistant PICsomes

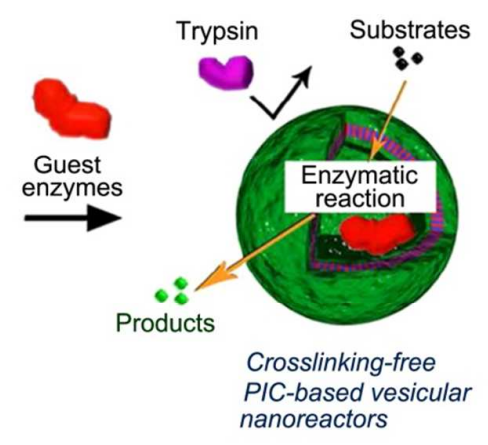

Figure 35. Schematic illustration of the formation of a salt- and thermal-resistant polyion complex vesicle as an enzymatic nanoreactor by encapsulating $\beta$-galactosidase in the core. ${ }^{26}$ (Reproduced with permission from reference 26. Copyright (2014) American Chemical Society).

In another effort to develop such enzyme-containing nanoreactors which only contain a single type of enzyme, more complex systems have been proposed involving two different cascade enzymes loaded inside nanoreactors ${ }^{260,293}$ or occupying different locations in the nanoreactor system (inside, within the membrane, or attached onto the membrane of the polymersome). ${ }^{292}$ For instance, van Hest and co-workers constructed biohybrid polymersome nanoreactors in which three different enzymes were spatially positioned, and precisely ordered (Figure 36a). ${ }^{292}$ These enzymes were incorporated into the membrane (Candida antarcticalipase B, CalB), encapsulated in the inner aqueous compartment (glucose oxidase, GO) and attached to the surface of the polymersome (horseradish peroxidase, HRP), respectively. Accordingly, the constructed nanoreactor was capable of performing a three-step cascade reaction, in which glucose acetate was converted by CalB to glucose, which 
was then oxidised by GO into gluconolactone in the second step. The hydrogen peroxide produced was used by HRP to oxidise 2,2'-azinobis(3-eth-ylbenzothiazoline-6-sulfonic acid) (ABTS) to green colour $\mathrm{ABTS}^{+}$.Furthermore, the authors also reported an interesting enzyme-filled Pickering emulsion that was stabilised by fully packed crosslinked polymersomes at the water/oil interface (Figure 36b-d). By loading a model enzyme CalBin different ways, either in the aqueous phase or inside the lumen of the polymersome to maximise the contact area at the interface between two phases, this polymersome-stabilised colloidosome architecture could be efficiently and repeatedly applied in biphasic enzymatic transformations. ${ }^{285}$
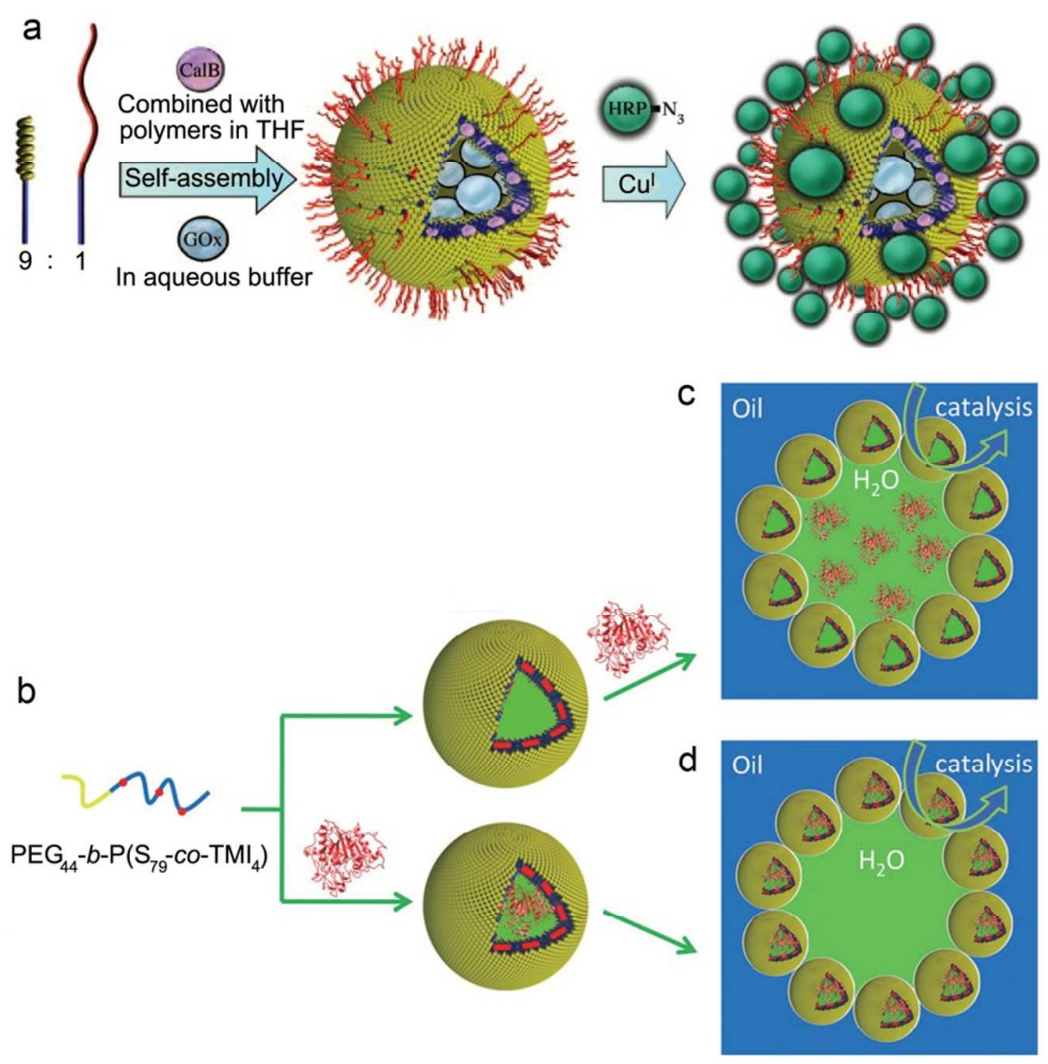

Figure 36. (a) Schematic representation of positional assembly of enzymes in a 
polymersome, with GO encapsulated in the inner compartment, CalB trapped in the polymeric bilayer and the third enzyme HRP immobilised on the polymersomal perimeter though a covalent linkage. ${ }^{292}$ (Reproduced with permission from reference 292. Copyright (2009) John Wiley and Sons). (b) Representation of the crosslinkingprocess to prepare stabilised polymersomes. (c) and (d) Schematic representation of a polymersome-stabilised Pickering emulsion with the enzyme in the water phase (c),or with the enzyme inside the polymersome lumen $(d){ }^{285}$ (Reproduced with permission from reference 285. Copyright (2012) John Wiley and Sons).

With the tremendously successful design of single compartments-based nanoreactors, investigations of the corresponding higher-order architectures, in particular bilayer-based multi-compartment capsules-based nanoreactors, is now gaining more and more attention. As illustrated in Figure $37 a$, a multi-compartmentalised nanoreactor was reported for that enzyme-filled polystyrene-b-poly(3-(isocyano-lalanyl-aminoethyl)thiophene) (PS-b-PIAT) nanoreactors were encapsulated together with free enzymes and substrates in a larger polybutadiene- $b$-poly(ethylene oxide) (PB- $b$-PEO) polymersome. ${ }^{86}$ By deliberate placing the enzymes needed for each separate cascade step in different subcompartments, the reaction intermediates were forced to cross over compartmental boundaries. Thereby, truly confined reactions and local or gradient-like generation of reaction products, as it occurs in nature, could be observed. In this pathway, the profluorescent substrate 1 (Figure 37b) first 
undergoes oxidation by the solvent- and temperature-stable Baeyer-Villiger monooxygenase, PAMO, which uses NADPH as a cofactor. The resulting ester moiety is then hydrolysed by $\mathrm{CalB}$, and subsequent oxidation of the resulting alcohol to an aldehyde is achieved by an $N A D^{+}$-dependent $A D H$. Finally, the last reaction yields fluorescent resorufin (5) through a spontaneous non-enzymatic beta-elimination step. ${ }^{86}$ Recently, Nallani and coworkers showed another construction of a multi-compartment polymersome nanoreactor where a passive protein channel OmpF was embedded into the inner membrane, forming a passive diffusion channel to allow for the exchange of small molecules and ions. ${ }^{269}$ Selective encapsulation of GO in the outer compartment and HRP in the inner compartment resulted in an efficient enzymatic cascade nanoreactor system. Such successful demonstration of this multi-compartment structure functionalised with membrane proteins is a significant step forward to tap into the selectivity displayed by such membranes, opening the possibility of engineering truly specialised multi-compartment nanoreactors. 


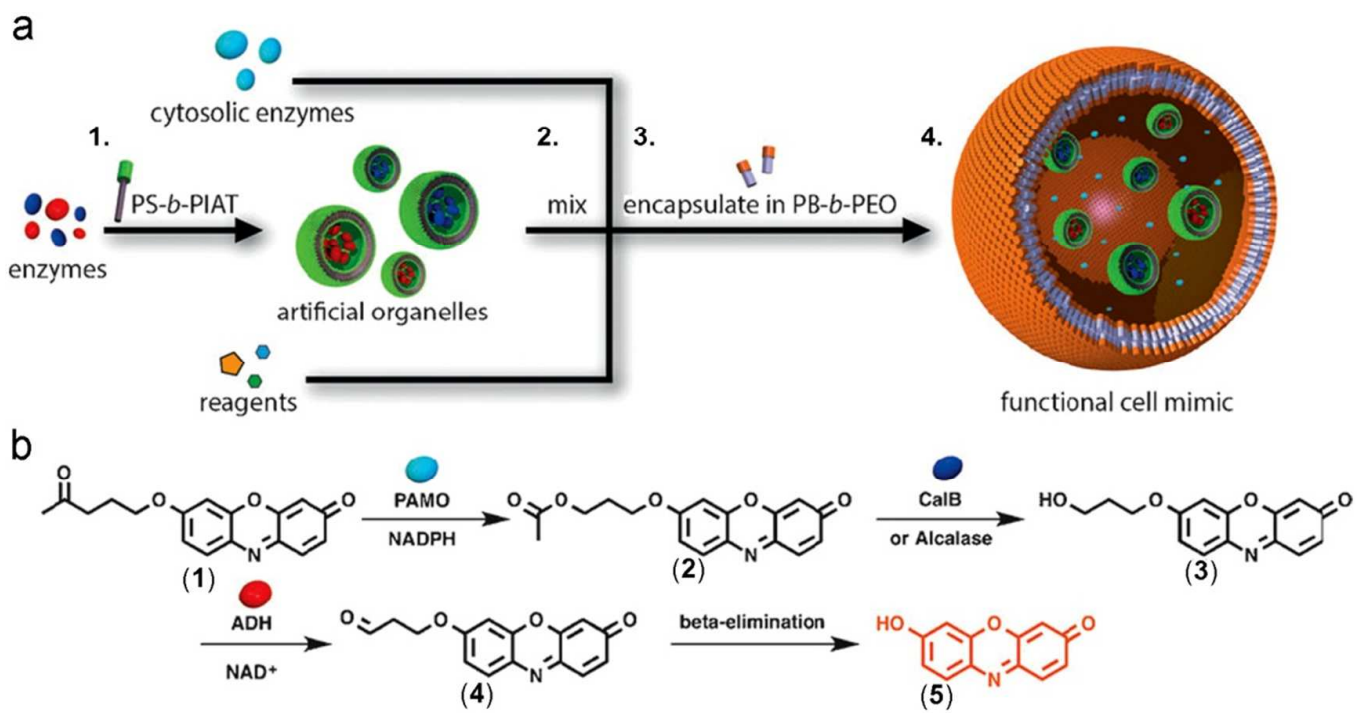

Figure 37. (a) Schematic illustration of the procedure to construct a multi-compartmentalised nanoreactor. The initial encapsulation of different enzymes in polystyrene-b-poly(3-(isocyano-lalanyl-amino-ethyl)-thiophene) (PS-b-PIAT) nanoreactors (1), followed by mixing of cytosolic enzymes, and other reagents (2), before encapsulation of the reaction mixture in polybutadiene- $b$-poly(ethylene oxide) (PB-b-PEO) vesicles (3) to create the multi-compartmentalised model (4), inside which enzymatic multi-compartment catalysis takes place. (b) A defined cascade reaction scheme happened in the constructed multi-compartmentalised model. ${ }^{86}$ (Reproduced with permission from reference 86. Copyright (2014) John Wiley and Sons).

Recently, other reports showed that apart from the application in catalysis, the constructed nanoreactors can also be used in theranostic approaches via installing fluorescent photo sensitiser signals and simultaneously producing reactive oxygen species(ROS) efficiently "on demand", 303 or can be used to protect the encapsulated guests from degrading agents and enzymes outside 
of the polymer capsules. ${ }^{281}$ This is especially important for biomedical applications of nanoreactors, where an encapsulated enzyme could produce a drug or scavenge toxic substances in intra- or extracellular fluids. ${ }^{283,291,304}$ For example, laccases (Lac) $)^{296}$ are oxidised enzymes with a broad range of applications, in soil remediation, as bleaching agent in the textile industry, and for cosmetics. Protecting laccases against degradation and inhibition is of great importance for many of these applications. With these objectives in mind, Bruns and coworkers demonstrated that encapsulation of Lac in the poly(N-vinylpyrrolidone)-block-poly(dimethylsiloxane)-block-poly(N-vinyl-pyrrol idone) (PNVP-b-PDMS-b-PNVP) triblock copolymers polymersomes significantly protected the enzyme against enzymatic degradation and against small inhibitors (Figure 38). ${ }^{296}$

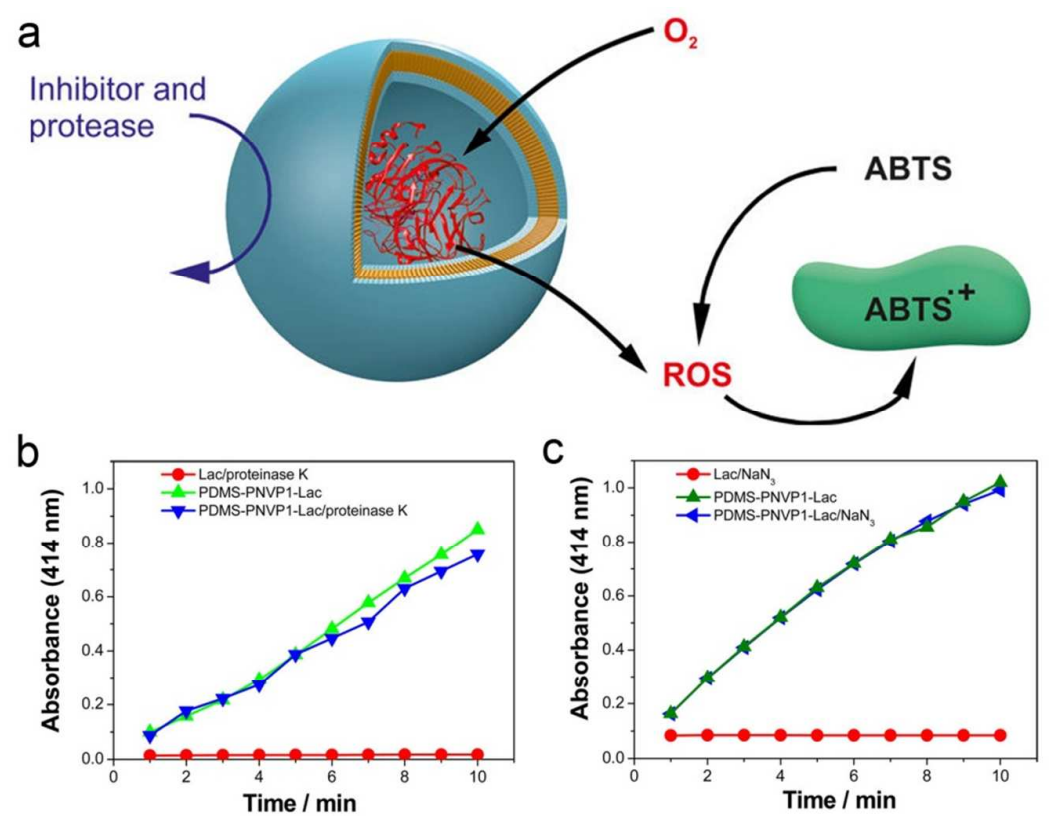

Figure 38.(a) Schematic illustration of a poly(N-vinylpyrrolidone)-poly(dimethylsiloxane)based polymersome nanoreactors for laccase-catalysed biotransformations. Protection of 
Lac in the PDMS-PNVP polymersomes against an externally added protease (b) and inhibitor $\mathrm{NaN}_{3}$ (c). ${ }^{296}$ (Reproduced with permission from reference 296. Copyright (2014) American Chemical Society).

\subsection{Application the Polymer Capsule Nanoreactors for Polymerisation}

Apart from the above-discussed incorporation of enzymes into the polymer capsules as nanoreactors, the hollow core domain itself has showed the ability to be an ideal confined reaction space for efficient polymerisation, ${ }^{305-307}$ and compartmentalization. For instance, physical confinement of reactants to monomer droplets or polymer capsules interior, enables the nanoreactors to show a marked beneficial effect on the progression of a controlled/living radical polymerisation based on the persistent radical effect. Up to now, although much work have been demonstrated in the field of polymerisation in nanoreactors, most of them are contingent on the radical polymerisation involving ATRP, RAFT or nitroxide-mediated radical polymerisation (NMP) taking place in emulsion, ${ }^{306}$ miniemulsion, ${ }^{308-309}$ or microemulsion. ${ }^{310}$ The influence of compartmentalisation on the kinetics of conventional radical emulsion polymerisation systems has been clearly demonstrated. The polymerisation rate and the obtained polymer molecular weight in an emulsion polymerisation are generally higher than in the corresponding homogeneous (bulk/solution) polymerisation, as a consequence of compartmentalisation of propagating radicals leading to reduced bimolecular termination rates. Two 
fundamental types of compartmentalisation effects were adopted: the segregation effect and the confined space effect. ${ }^{311}$ The segregation effect refers to two species located in separate particles being unable to react, and the confined space effect refers to two species located in the same compartment reacting at a higher rate in a small space than in a large space. Overall, the segregation effect on bimolecular termination results in an increase in livingness (higher end-functionality), whereas the confined space effect on deactivation may result in an increase in the level of control over the molecular weight distribution.

For example, Zhang and co-workers presented a study about RAFT polymerisation of a typical hydrophobic monomer of styrene within a hollow capsule suspended in water (Figure 39). ${ }^{307}$ A hollow capsule containing a hydrophilic corona of poly(methacrylic acid) (PMAA) and a cross-linked polystyrene shell was synthesized within which the typical hydrophobic monomer of styrene, the RAFT agent S-benzyldithiobenzoate and the initiator 2,2'-azobisisobutyronitrile can be encapsulated and RAFT polymerisation of styrene took place in well controlled manner in water. In comparison of the homogeneous bulk polymerisation, it is found that the resultant polymer of polystyrene has a competitively low dispersity index and its number-average molecular weight linearly increases with monomer conversion. Altogether, as outlined in the reported models, compartmentalisation in nanoreactors of living radical polymerisation, as long as the deactivator concentration is sufficiently 
low, can offer a means of improving both the level of control over the molecular weight distribution and the end-functionality.

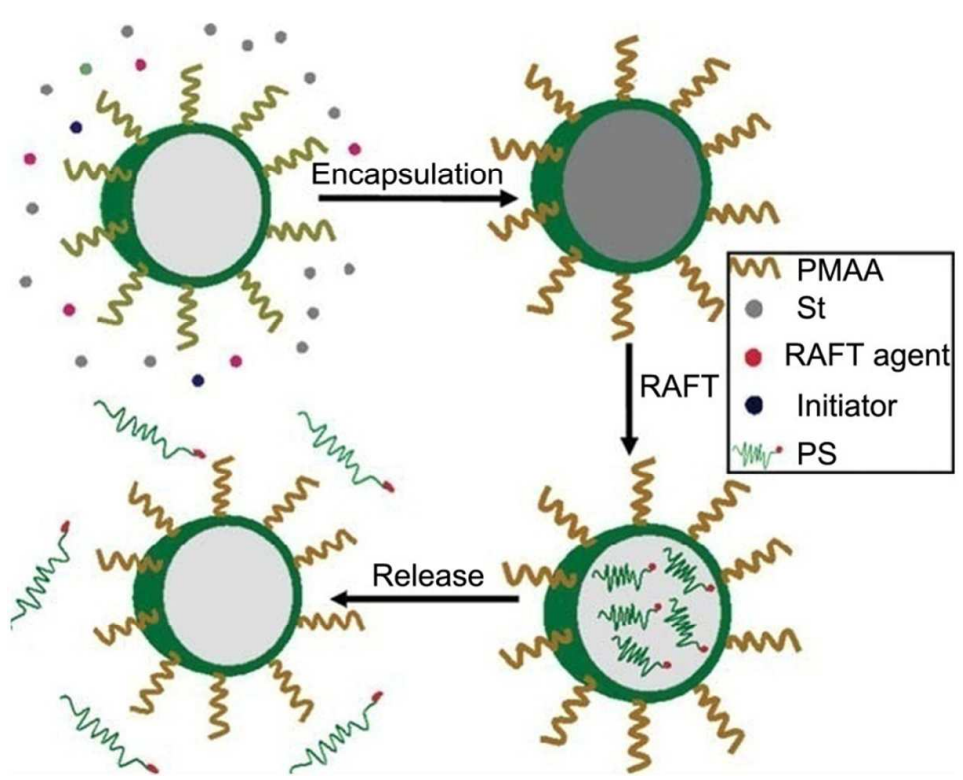

Figure 39. Schematic nanoreactors formation when-RAFT polymerisation of styrene in water is used. ${ }^{307}$ (Reproduced with permission from reference 307. Copyright (2010) Wiley Periodicals, Inc.).

\subsection{Application of the Polymer Capsule Nanoreactors for Nanoparticles Synthesis}

Some reports demonstrated that polymer capsules-based nanoreactors can also be exploited for promoting nanoparticles synthesis, since the empty core domain has shown the ability to carry reagents and that it was able to create a favourable environment to optimise the reaction. In this regard, microemulsions should be one of the first types of individual microreactors explored for nanosynthesis. Different preparation procedures to fabricate both direct and reverse emulsions have been developed and studied. ${ }^{312-315}$ These 
procedures allow the preparation of monodisperse, stable microemulsions of different sizes. Currently, a wide range of inorganic nanoparticles have been obtained in microemulsion systems involving the preparation of $\mathrm{SiO}_{2},{ }^{315-316}$ $\mathrm{TiO}_{2}^{317}$ or $\mathrm{CaCO}_{3}^{318}$ etc. in reverse water-in-oil emulsions. Despite the significant success in spatially confined nanosynthesis inside emulsions, alternatively, polymer capsules such as constructs based on layer-by-layer strategy, as another elegant system for catalysing nanosynthesis was also developed. ${ }^{319}$ One of the significant advantages of this polymer capsule is the semipermeable shell which is permeable for small ions but not permeable for polymers or larger particles. ${ }^{320}$ In view of the fact that some polymers can suppress the growth of inorganic crystals, this allows the formation of inorganic particle strictly inside the core domain of the capsules keeping the suppressing polymers outside. ${ }^{314}$ Thus, the growth of calcium carbonate and barium carbonate particles only inside the polymer capsules was obtained.

Another way to initiate the growth of particles in the capsule interior is to change $\mathrm{pH}$ of the whole system slightly over the threshold of solubility. The inner shell of the capsules might serve for nucleation. ${ }^{321}$ It is possible to synthesize functional materials in the capsule, and to modify the permeation properties of the polymer capsule walls by deposition polymers within the capsule walls. ${ }^{322}$ Moreover, Sukhorukov and coworkers also demonstrated the influence of $\mathrm{pH}$ gradient on the chemical reaction in the capsule interior. The precipitation of acidic dyes in a polymer capsule filled with negatively charged 
poly(styrene sulfonate) (PSS) buffering the $\mathrm{pH}$ around 2-2.5 was studied. ${ }^{323}$ Once the carboxylic dyes went through the membrane, the low $\mathrm{pH}$ inside the capsules resulted in precipitation. Accordingly, the capsules were filled with dye molecules. Based on such strategy, the successful synthesis of other inorganic materials including polytungstic acid and nanosized $\mathrm{WO}_{3}$ in the capsules was demonstrated. ${ }^{324}$ In contrast, the authors also showed that if using cationic poly(allyl amine hydrochloride) (PAH) instead of anionic PSS, an alkaline environment was created inside the capsule which was able to catalyse the synthesis of nanosized $\mathrm{Fe}_{2} \mathrm{O}_{3}, \mathrm{TiO}_{2}$ gel or magnetic ferrites within the capsule interior. ${ }^{325-326}$

\subsection{Artificial Organelles}

Cell organelles are membrane-delineated intracellular components with specialised functions. ${ }^{29,34-35}$ They have unique biochemical microenvironments, characterised by a specific set of enzymes, and are enclosed by a selective membrane, allowing the subcellular spatial control of different functions. They are thus responsible for crucial cellular activities, for example mitochondria are involved in cellular respiration, ribosomes in protein production etc. Recently, the design of simplified, synthetic organelles capable of functioning inside of cells for the purpose of both producing drugs and enhancing the function of natural organelles in pathological conditions by using small surfactant such as 
a lipid as a building block has gathered considerable interest. ${ }^{327-328}$ In this respect, it is not surprising that polymer capsules, in consideration of their aqueous cavities which can serve as a reaction space, ${ }^{329}$ and the semipermeable membrane which allows transport of necessary substrates and products of the enzymatic reactions, have been taken as one of the most popular scaffolds to develop artificial organelles. Up to now, pioneering attempts have been made by Meier and von Hest et. al. to exploit polymer compartments into artificial organelles by loading enzymes or mimics of them inside (Figure 40). Meanwhile, basic requirements to design artificial organelles have been concluded: ${ }^{28,33}$ (i) polymer compartments of appropriate size for cell-uptake, (ii) activity of encapsulated enzymes maintained in situ, (iii) a polymer membrane, which is stable and permeable to small molecules, (iv) integrity and activity preserved in cells, and ( $v$ ) biocompatibility and biodegradability of polymer compartments.
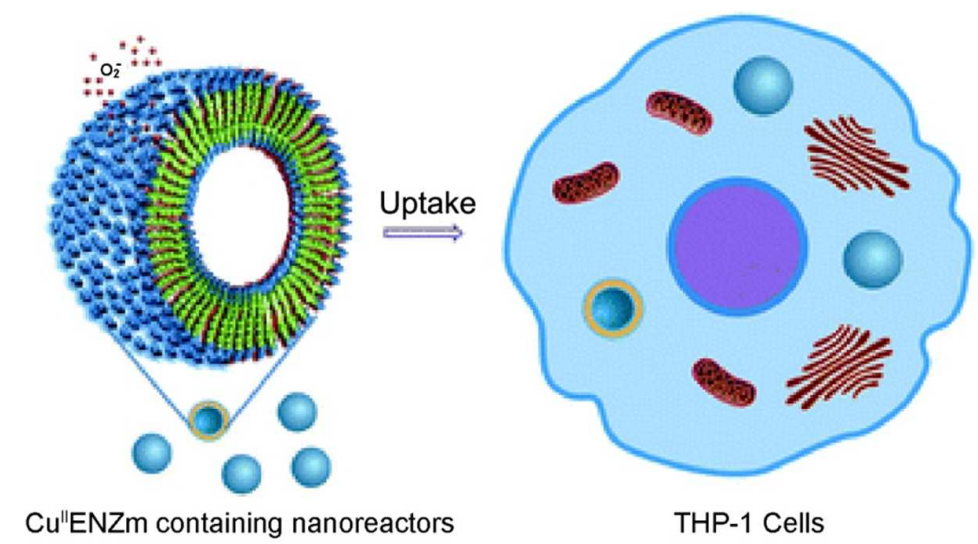

Figure 40. Schematic representation of the construction of polymeric nanoreactors towards artificial organelles by incorporating a dual-enzyme mimic of superoxide 
dismutase and catalase. The uptake of these loaded polymeric nanoreactors into THP-1 cells. ${ }^{167}$ (Reproduced with permission from reference 167. Copyright (2011) Royal Society of Chemistry).

With these objectives in mind, Meier and Palivan showed a series of beautiful work on exploiting polymersome nanoreactors into artificial organelles by encapsulating enzymes/enzyme mimics into the core. ${ }^{30,156,166-167}$ As discussed in section 3.1.1 (Figure 20), the successful construction of an artificial model represents a milestone in the development of in vivo cell implants and a new frontier in medical therapy. Undoubtedly, this encourages researchers for designing various artificial organelles. Recently, also van Hest and coworkers made a great effort to exploit enzyme-loaded polymersomes into artificial organelles. ${ }^{35}$ As illustrated in Figure $41,{ }^{289}$ a horseradish peroxidase-filled polymersome was presented, which was capable of protecting its contents from proteolytic degradation. ${ }^{289-290}$ To promote the cellular uptake, cell-penetrating peptides were covalently anchored onto the surface of the model. It was found that the cellular delivery of polymersomes was restricted to cells with an intrinsically high phagocytic activity, and the catalytic activity conferred to the cells was maintained at levels that were significantly higher than those reported for soluble enzymes. 


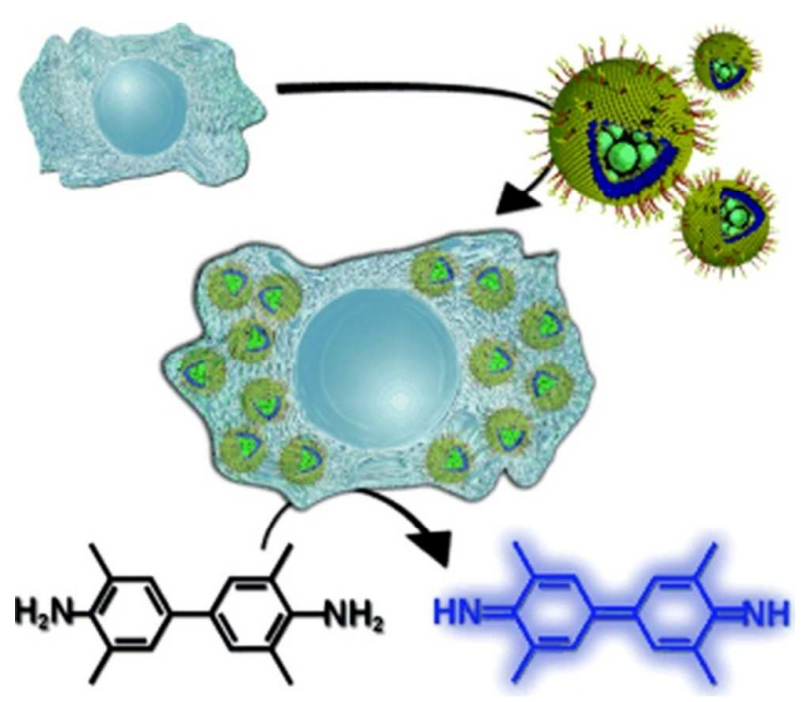

Figure 41. Schematic illustration of a polystyrene-block-poly[L-isocyanoalanine (2-thiophen-3-ylethyl) amide] (PS-PIAT)-based polymersome nanoreactor functionalised with Tat-peptide. This protein is known to mediate cellular uptake and thus also to the artificial organelle. Encapsulated HRP converts the substrate tetramethylbenzidine, which allows the activity of the nanoreactor to be measured by absorption. ${ }^{289}$ (Reproduced with permission from reference 289. Copyright (2010) John Wiley and Sons).

Last but not least, it is interesting to mention that a cellular Trojan horse acting as a source of reactive oxygen species (ROS) "on demand" inside cells was successfully reported by Palivan et al.(Figure 42). ${ }^{330}$ The Trojan horse was engineered by optimizing a light responsive model based on a highly active photosensitiser encapsulated in two different amphiphilic block copolymers (PMOXA-PDMS-PMOXA and PNVP-PDMS-PNVP) polymersomes. The models based onPMOXA-PDMS-PMOXA polymersomes with diameters of around $200 \mathrm{~nm}$ were the most efficient in terms of photo 
sensitiser encapsulation and size to fulfil the requirements for high retention and permeability. These constructed models also showed the highest uptake and stability in HeLa cells. While nontoxic in dark conditions, they produced ROS upon illumination in conditions specific for photodynamic therapy. Intracellular ROS levels increased by a factor of 40 , which was sufficient to significantly reduce cell viability in the region containing illuminated Trojan horse models.

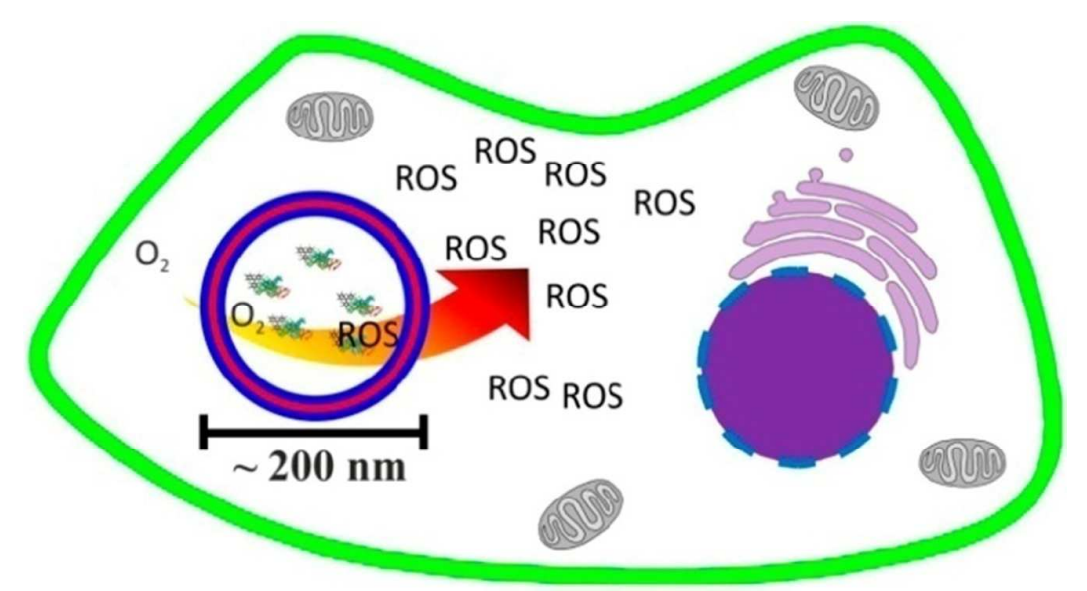

Figure 42. Schematic representation of a light-responsive nanoreactor containing photosensitisers to act as a cellular Trojan horse by producing ROS inside cells "on demand". ${ }^{330}$ (Reproduced with permission from reference 330. Copyright 2014, American Chemical Society).

Despite tremendous efforts and the existence of exciting synthetic models, the studies reported on the application of polymer capsule-based nanoreactors as actual artificial organelles in vitro and eventually in vivo, are still mainly 
proofs of concept, and there are many challenges ahead before the full realisation of the implantation in cells to treat pathological conditions or to support the design of artificial cells will be seen. As major prerequisites which need to be realized in polymeric capsules as artificial organelles, introduction of specific targeting of cell types of interest and subsequent effective internalised by those cells have to be named. Furthermore, the designed models should be biocompatible without causing side effects by interactions with cells. Also, localisation of artificial organelles after cellular uptake is still an issue of intense research, given that polymersomes are reported to be routed to endosomes and lysosomes after internalisation. Escaping from the endocytotic machinery is therefore another key step towards fully functional artificial organelles.

\section{Conclusions}

In this review, we have put a spotlight on recent developments in polymer capsules with special emphasis on the field of polymersomes and their use as nanoreactors. After the first publications of Discher and Eisenberg on polymer vesicles in 1999 and 2002,, 2,14 the field has advanced rapidly. There are now multiple ways to prepare vesicles from amphiphilic block-copolymers in a defined way and further equip them with multiple functionalities and responsiveness to realize effective drug delivery systems, nanoreactors and even artificial organelles. 
Polymersome-making procedures are now available for functional and non-functional block-copolymers, being rubbery, of glassy nature, or crystalline, covering film rehydration, solvent, $\mathrm{pH}$, or temperature switch, as well as emulsion, centrifugation or microfluidic techniques. This variety in methods allows for targeted and highly effective polymersome production, whilst avoiding or minimizing other aggregates like micelles and worm-like and tubular vesicles. The latest development in this respect, the formation of vesicles during polymerisation (so-called PISA) now even allows for in-situ vesicle production. Altogether one can conclude that it is now possible, by choosing the fitting method, to prepare vesicles of all amphiphilic block-copolymers which have the correct block-length ratio, regardless of the exact polymer composition involved. Recent developments in proteinosomes and dendrimersomes are very good examples in that respect. Especially proteinosomes caught up very quickly with their counterparts of purely artificial polymers. The future development of methods faces the challenge to be more robust or quicker than the current ones, if they are to be established in the field.

The preparation methods for polymer capsules not requiring amphiphilic block-copolymers of the right ratio, like microfluidics and template methods, are also briefly highlighted. Here, the main challenge is to remove the central core without damaging the polymer membrane. Success in this regard is reported but clearly polymersomes dominate so far the field of nanoreactors. 
The main reason is that functionality of almost any kind can easily be included into polymersomes to make the membrane responsive and permeable. The inclusion of transmembrane proteins for this purpose is developed to a stage that cellular organelles can be mimicked and also replaced. Especially the development of $\mathrm{pH}$ sensitive polymersomes is very popular. The introduction of a variety of tertiary amines and acetal cleavages gives $\mathrm{pH}$ (or sometimes $\mathrm{CO}_{2}$ ) sensitive systems with some variety of the pKa, which is even more diverse if boronic acid is taken into account as well. Temperature sensitive systems have also been realized and are recently more driven towards application. Also a great variety of cross-linking methods have been developed for stabilizing the capsule membrane. They cover photochemical, radical and condensation reactions as well as physical cross-linking. Cross-linked systems are often combined with redox, temperature and $\mathrm{pH}$ sensitive function to give vesicles with reversible membrane swelling.

In this review we focused on the application of polymersomes, or more general polymeric capsules, as nanoreactors. For a nanoreactor to work, an enzyme or any other active cargo need to be enclosed and the substrate and reagents need to reach it. This principle as now available for cross-linked and non-cross-linked vesicles to give nanoreactors of high complexity allowing for multi-component and multi-stage reaction cascades. Most prominent are the development of artificial cell organelles, vesicle-in-vesicle nanoreactors and reactions across different vesicles. Today, vesicles can also be used as 
nanoreactors to produce new polymers or to enhance polymerisation procedures, making this the starting point of self-replicating polymersomes.

Cleary, exiting progress has been made in the recent years in engineering functional polymer capsules towards smart nanoreactors. High potential can be seen, e.g., in exploiting biohybrid approaches as in the reported proteinosomes mimicking even better biological processes and selective reaction schemes. Still, more robust preparation procedures with the potential for scale-up and further application studies beyond the proof of principles shown so far are needed to fully exploit the high promise of polymer capsule based nanoreactors in biomedicine, bio-catalysis or biotechnology.

\section{Acknowledgements}

We thank the Deutsche Forschungsgemeinschaft (DFG) for supporting JG (Grant GA 2051/1-1) and both, the National Natural Science Foundation of China (21474025) as well as 1000 Young Talent Program for supporting $\mathrm{XH}$

\section{Table 1: List of polymer structures}

\begin{tabular}{|c|c|c|c|}
\hline $\begin{array}{l}\mathbf{W} / \\
\mathbf{L}^{l}\end{array}$ & Name & $\begin{array}{l}\text { Abbreviation/ } \\
\text { Property }\end{array}$ & Formula \\
\hline W & Poly(ethylene $\quad$ oxide) & PEO or PEG & $\mathrm{H}_{\mathrm{O}} \curvearrowright \mathrm{f}_{\mathrm{n}} \mathrm{O}_{\mathrm{H}}$ \\
\hline
\end{tabular}




\begin{tabular}{|c|c|c|c|}
\hline & orPoly (ethylene glycol) & Non-Immunogenic & \\
\hline W & $\begin{array}{l}\text { Poly(methacrylic } \\
\text { phosphoryl choline) }\end{array}$ & $\begin{array}{l}\text { PMPC } \\
\text { Non-Immunogenic }\end{array}$ & 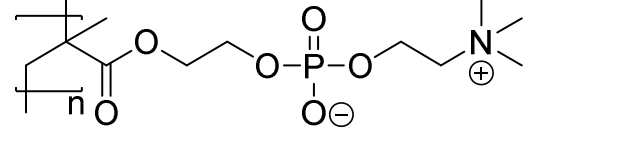 \\
\hline $\mathrm{W}$ & $\begin{array}{l}\text { Poly(oligo(ethylene } \\
\text { glycol)methacrylate) }\end{array}$ & $\begin{array}{l}\text { POEGMA } \\
\text { Non-Immunogenic } \\
\text { PDEGMEMA for } 2 \\
\text { PEG units }\end{array}$ & | $8-10$ \\
\hline $\mathrm{W}$ & $\operatorname{Poly}(($ meth$)$ acrylic acid $)$ & $\mathrm{P}(\mathrm{M}) \mathrm{AA}$ & PAA $-X=\mathrm{H}, \mathrm{PMAA}-\mathrm{X}=\mathrm{Me}$ \\
\hline $\mathrm{W}$ & Poly(methyl oxazoline) & $\begin{array}{l}\text { PMOxA } \\
\text { Non-Immunogenic } \\
\text { PEtOxa - Ethyl } \\
\text { derivative }\end{array}$ & $\mathrm{O} M e(E \mathrm{t})$ \\
\hline $\mathrm{W}$ & $\begin{array}{l}\text { Poly(L - isocyanoalanine( } 2 \\
\text { - thio- phen - } 3 \text { - yl - } \\
\text { ethyl)amide) }\end{array}$ & PIAT & \\
\hline $\mathrm{W}$ & $\begin{array}{l}\text { Poly(N-isopropylacrylamid } \\
\text { e) }\end{array}$ & $\begin{array}{l}\text { PNIPAM, } \\
\text { Temperature } \\
\text { sensitive }\end{array}$ & $\vec{H}$ \\
\hline
\end{tabular}




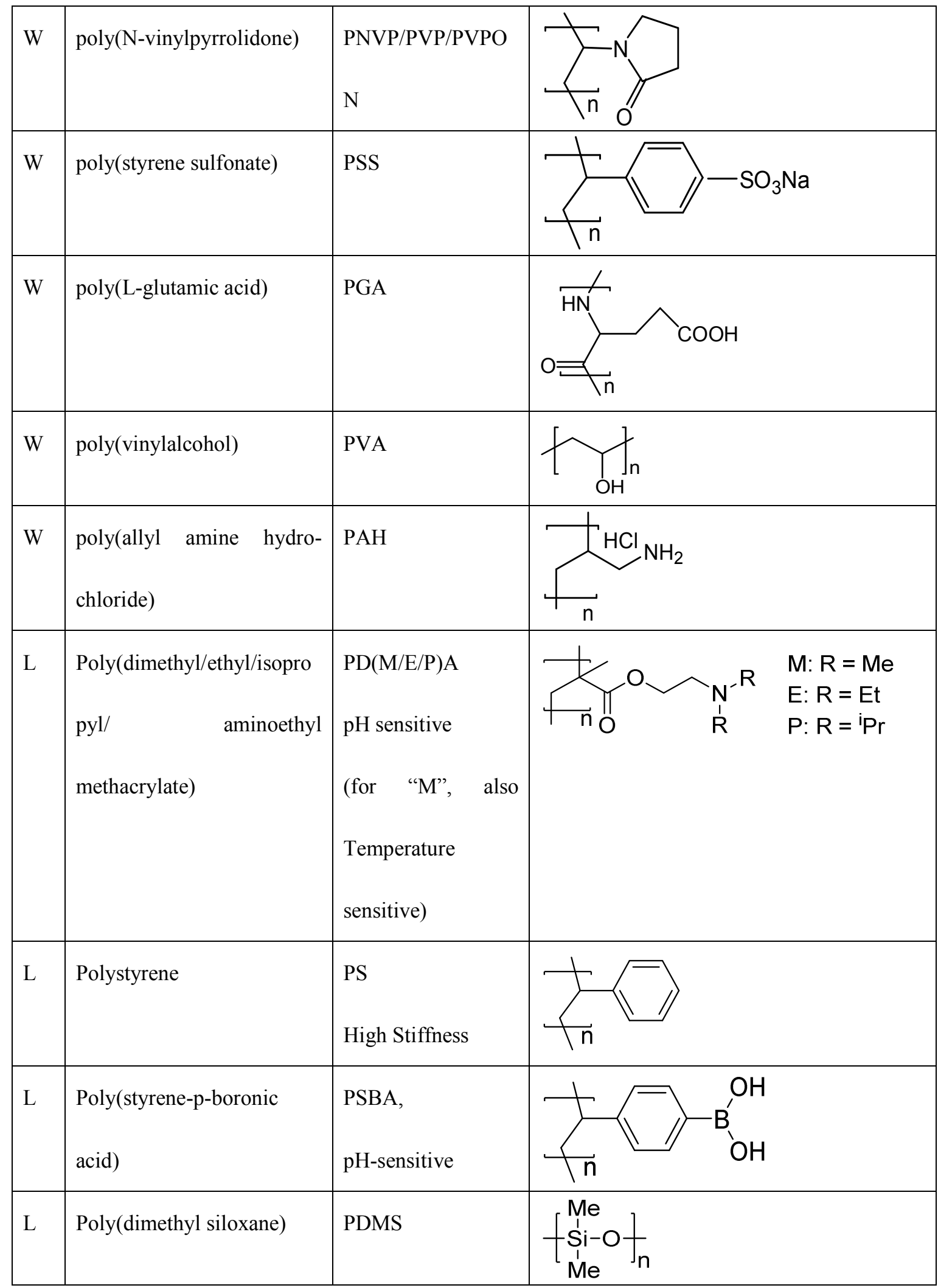




\begin{tabular}{|c|c|c|c|}
\hline $\mathrm{L}$ & Poly(benzyl-L-glutamate) & PBLG & {$\left[\begin{array}{ll}O & H \\
N & H\end{array}\right]$} \\
\hline $\mathrm{L}$ & $\begin{array}{l}\text { Poly(dimethylmaleicimido( } \\
\text { ethyl/butyl) methacrylate) }\end{array}$ & $\begin{array}{l}\text { PDMI(E/B)M } \\
\text { Photo-Cross-linkab } \\
\text { le }\end{array}$ & $E-m=1, B-m=2$ \\
\hline $\mathrm{L}$ & $\begin{array}{l}\text { Poly(cinnamoylethylmeth- } \\
\text { acrylate) }\end{array}$ & PCEMA & $+<$ \\
\hline $\mathrm{L}$ & Polycaprolactone & $\begin{array}{l}\text { PCL, } \\
\text { Biodegradable }\end{array}$ & H \\
\hline $\mathrm{L}$ & Poly(propylene) glycol & PPG & \\
\hline $\mathrm{L}$ & $\begin{array}{l}\text { (trans-N-(2-ethoxy-1,3-diox } \\
\text { an-5-yl) acrylamide)unit }\end{array}$ & $\mathrm{t}(\mathrm{NEA})$ & \\
\hline $\mathrm{L}$ & Poly(L-)lactic acid & $\begin{array}{l}\text { PLLA, } \\
\text { Biodegradable } \\
\text { High Stiffness }\end{array}$ & \\
\hline $\mathrm{L}$ & Polybutadiene & $\begin{array}{l}\text { PBD (PB) } \\
\text { Can be } \\
\text { Hydrogenated }\end{array}$ & \\
\hline $\mathrm{L}$ & Poly(propylene sulphide) & $\begin{array}{l}\text { PPS } \\
\text { Oxidation-sensitive }\end{array}$ & $\left.\mathrm{S}_{\mathrm{s}}\right\}_{\mathrm{n}}^{\mathrm{H}}$ \\
\hline
\end{tabular}




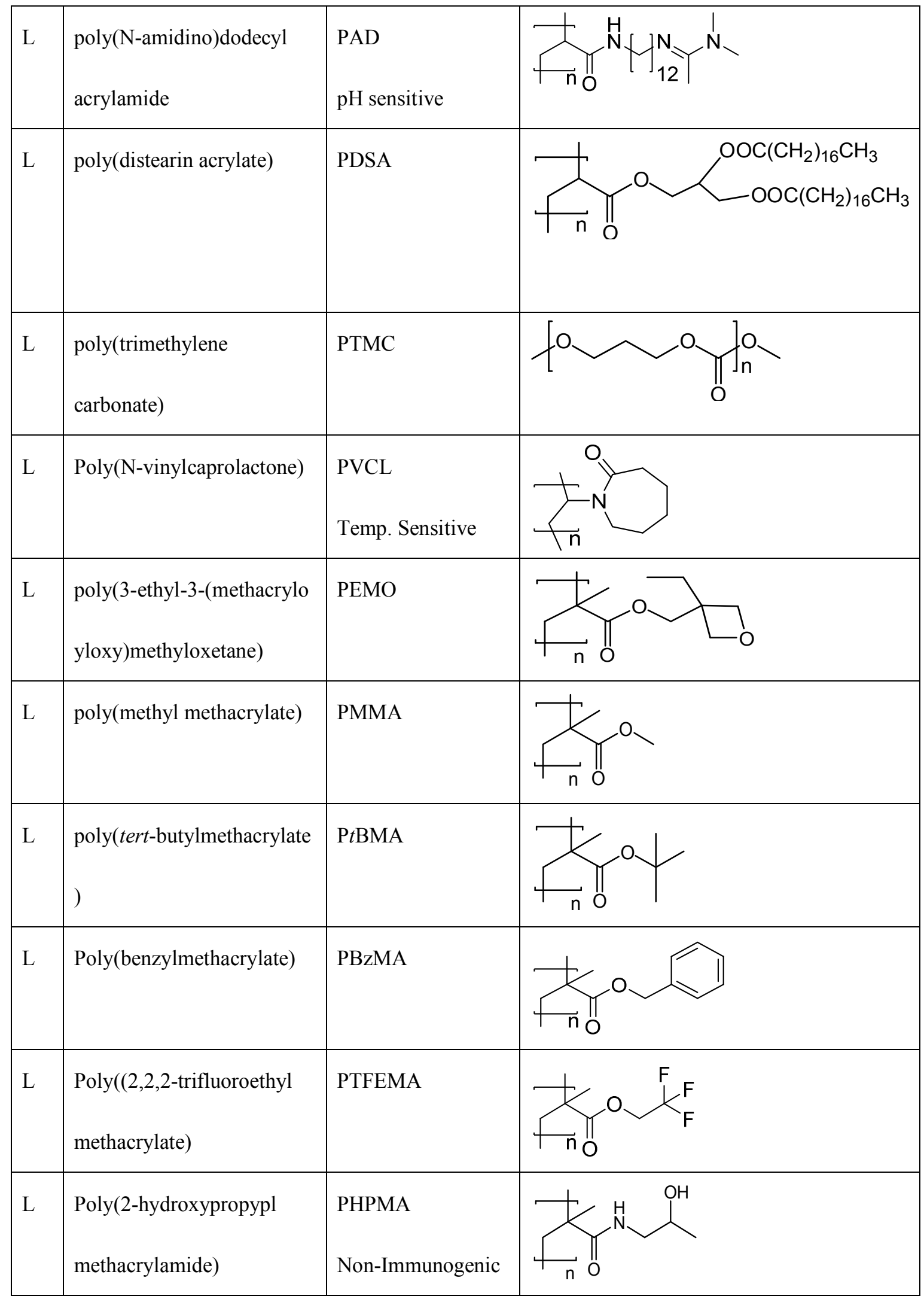




\begin{tabular}{|l|l|l|l|l|}
\hline $\mathrm{L}$ & $\begin{array}{l}\text { Poly(n-butyl methacrylate) } \\
\text { poly(lauryl methacrylate) }\end{array}$ & PBMA, $\mathrm{PLMA}=2$ \\
\hline $\mathrm{L}$ & $\begin{array}{l}\text { Poly(histidine) } \\
\text { protect- ted) }\end{array}$ & (trityl & Phis(-Trt) \\
\hline $\mathrm{L}$ & Azobenzene-containing & $\begin{array}{l}\text { Dendritic unit } \\
-\mathrm{O}\end{array}$ \\
dendrons & $\mathrm{O}$
\end{tabular}

${ }^{1} \mathrm{~W}=$ Hydrophilic (Water soluble) / L = Hydrophobic (Lipid soluble)

\section{Abbreviations}

\begin{tabular}{|l|l|}
\hline ABTS & 2,2'-Azinobis(3-eth-ylbenzothiazoline-6-sulfonic acid) \\
\hline AGET ATRP & Electron transfer atom transfer radical polymerisation \\
\hline AIBN & $2,2^{\prime}$-Azobisisobutyronitrile \\
\hline ATP/ADP & Adensonin tri(di-)phosphate \\
\hline ATRP & Atom transfer radical polymerisation \\
\hline BR & Bacterial rhodopsin \\
\hline CAC & Critical aggregate concentration \\
\hline CalB & Candida antarcticalipase B \\
\hline CDB & Phenyl 2-propyl dithiobenzoate \\
\hline
\end{tabular}




\begin{tabular}{|c|c|}
\hline CTA & Chain transfer agent \\
\hline DBTTC & Dibenzyltrithiocarbonate \\
\hline DEAEMA & Diethylaminoethyl methacrylate \\
\hline DNA & Desoxyribonucleic acid \\
\hline DODAB & Dimethyldioctadecylammonium bromide \\
\hline DTT & Dithiothreitol \\
\hline GA & Glucose amylase, \\
\hline GFP & Green fluorescent protein \\
\hline GO & Glucose oxidase \\
\hline GSH & Glutathione \\
\hline GSSG & Glutathione disulphide \\
\hline HRP & Horseradish peroxidase \\
\hline ITO & Indium tin oxide \\
\hline KPS & Potassium persulfate \\
\hline Lac & Laccase \\
\hline LCST & Lower critical solution temperature \\
\hline MES & 2-(N-morpholino)ethanesulfonic acid buffer \\
\hline NADPH & Nicotinamide adenine dinucleotide phosphate \\
\hline NCA & N-carboxyanhydrid \\
\hline NMRP & Nitroxide mediated radical polymerisation \\
\hline OmpF & Outer-membrane-protein F \\
\hline PBS & Phosphate buffered saline \\
\hline
\end{tabular}




\begin{tabular}{|l|l|}
\hline PDSM & Pyridyldisulphideethylmethacrylate \\
\hline PISA & Polymerisation induced self-assembly \\
\hline PPPDTA & Phenyl 2-propyl phenyl dithioacetate \\
\hline RAFT & Reversible addition-fragmentation chain-transfer \\
\hline ROS & Reactive oxygen species \\
\hline ROP & Ring opening polymerisation \\
\hline SIP & Surface-initiated polymerisation \\
\hline TEM & Transmission electron microscopy \\
\hline TVNH & Vivax nucleoside hydrolyse \\
\hline
\end{tabular}

\section{References}

(1) Mann, S. "Systems of Creation: The Emergence of Life from Nonliving Matter" Acc. Chem. Res. 2012, 45, 2131-2141.

(2) Discher, D. E.; Eisenberg, A. "Polymer vesicles" Science 2002, 297, 967-973.

(3) Schwille, P. "Bottom-up synthetic biology: engineering in a tinkerer's world" Science 2011, $333,1252-1254$.

(4) Szostak, J. W.; Bartel, D. P.; Luisi, P. L. "Synthesizing life" Nature 2001, 409, 387-390.

(5) Dinsmore, A. D.; Hsu, M. F.; Nikolaides, M. G.; Marquez, M.; Bausch, A. R.; Weitz, D. A. "Colloidosomes: Selectively Permeable Capsules Composed of Colloidal Particles" Science 2002, 298, 1006-1009.

(6) Meier, W. "Polymer nanocapsules" Chem. Soc. Rev. 2000, 29, 295-303. 
(7) Caruso, F.; Caruso, R. A.; Möhwald, H. "Nanoengineering of inorganic and hybrid hollow spheres by colloidal templating" Science 1998, 282, 1111-1114. Utada, A. S.; Lorenceau, E.; Link, D. R.; Kaplan, P. D.; Stone, H. A.; Weitz, D. A. "Monodisperse Double Emulsions Generated from a Microcapillary Device" Science 2005, $308,537-541$.

(9) Blanazs, A.; Armes, S. P.; Ryan, A. J. "Self-Assembled Block Copolymer Aggregates: From Micelles to Vesicles and their Biological Applications" Macromol. Rapid Commun. 2009, 30, 267-277.

(10) Renggli, K.; Baumann, P.; Langowska, K.; Onaca, O.; Bruns, N.; Meier, W. "Selective and Responsive Nanoreactors" Adv. Funct. Mater. 2011, 21, 1241-1259.

(11) Letchford, K.; Burt, H. "A review of the formation and classification of amphiphilic block copolymer nanoparticulate structures: micelles, nanospheres, nanocapsules and polymersomes" Eur. J. Pharm. Biopharm. 2007, 65, 259-269.

Kisak, E.; Coldren, B.; Evans, C.; Boyer, C.; Zasadzinski, J. "The vesosome-a multicompartment drug delivery vehicle" Curr. Med. Chem. 2004, 11, 199-219.

(13) Pearson, R. T.; Warren, N. J.; Lewis, A. L.; Armes, S. P.; Battaglia, G. "Effect of pH and temperature on PMPC-PDPA copolymer self-assembly" Macromolecules 2013, 46, $1400-1407$.

(14) Discher, B. M.; Won, Y. Y.; Ege, D. S.; Lee, J. C. M.; Bates, F. S.; Discher, D. E.; Hammer, D. A. "Polymersomes: Tough vesicles made from diblock copolymers" Science 1999, 284, 1143-1146.

(15) Percec, V.; Wilson, D. A.; Leowanawat, P.; Wilson, C. J.; Hughes, A. D.; Kaucher, M. S.; 121 
Hammer, D. A.; Levine, D. H.; Kim, A. J.; Bates, F. S.; Davis, K. P.; Lodge, T. P.; Klein, M. L.; DeVane, R. H.; Aqad, E.; Rosen, B. M.; Argintaru, A. O.; Sienkowska, M. J.; Rissanen, K.; Nummelin, S.; Ropponen, J. "Self-Assembly of Janus Dendrimers into Uniform Dendrimersomes and Other Complex Architectures" Science 2010, 328, 1009-1014.

Huang, X.; Li, M.; Green, D. C.; Williams, D. S.; Patil, A. J.; Mann, S. "Interfacial assembly of protein-polymer nano-conjugates into stimulus-responsive biomimetic protocells" Nat. Commun. 2013, 4, 3239, DOI: 2210.1038/ncomms3239.

Vargo, K. B.; Parthasarathy, R.; Hammer, D. A. "Self-assembly of tunable protein suprastructures from recombinant oleosin" Proc. Natl. Acad. Sci. U.S.A 2012, 109, $11657-11662$.

(18) Marguet, M.; Bonduelle, C.; Lecommandoux, S. "Multicompartmentalized polymeric systems: towards biomimetic cellular structure and function" Chem. Soc. Rev. 2013, 42, 512-529.

(19) Huang, X.; Voit, B. "Progress on multi-compartment polymeric capsules" Polym. Chem. 2013, $4,435-443$.

(20) Messager, L.; Gaitzsch, J.; Chierico, L.; Battaglia, G. "Novel aspects of encapsulation and delivery using polymersomes" Curr. Opin. Pharmacol. 2014, 18, 104-111.

(21) Battaglia, G.; Ryan, A. J. "Pathways of polymeric vesicle formation" J. Phys. Chem. B 2006, $110,10272-10279$.

(22) Wang, S.; Wang, H.; Liu, Z.; Wang, L.; Wang, X.; Su, L.; Chang, J. "Smart pH- and reduction-dual-responsive folate-PEG-coated polymeric lipid vesicles for tumor-triggered targeted drug delivery" Nanoscale 2014, 6, 7635-7642.

(23) Lv, L.-P.; Landfester, K.; Crespy, D. "Stimuli-Selective Delivery of two Payloads from Dual 
Responsive Nanocontainers" Chem. Mater. 2014, 26, 3351-3353.

(24) Ru, C.; Fenghua, M.; Chao, D.; Klok, H. A.; Zhiyuan, Z. "Dual and multi-stimuli responsive polymeric nanoparticles for programmed site-specific drug delivery" Biomaterials 2013, 34, $3647-3657$.

Kost, J.; Langer, R. "Responsive polymeric delivery systems" Adv. Drug Delivery Rev. 2012, $64,327-341$.

(26) Egli, S.; Schlaad, H.; Bruns, N.; Meier, W. "Functionalization of Block Copolymer Vesicle Surfaces" Polymers 2011, 3, 252-280.

Chuanoi, S.; Anraku, Y.; Hori, M.; Kishimura, A.; Kataoka, K. "Fabrication of Polyion Complex Vesicles with Enhanced Salt and Temperature Resistance and Their Potential Applications as Enzymatic Nanoreactors" Biomacromolecules 2014, 15, 2389-2397.

(28) Dobrunz, D.; Toma, A. C.; Tanner, P.; Pfohl, T.; Palivan, C. G. "Polymer Nanoreactors with Dual Functionality: Simultaneous Detoxification of Peroxynitrite and Oxygen Transport" Langmuir 2012, 28, 15889-15899.

(29) Tanner, P.; Baumann, P.; Enea, R.; Onaca, O.; Palivan, C.; Meier, W. "Polymeric Vesicles: From Drug Carriers to Nanoreactors and Artificial Organelles" Acc. Chem. Res. 2011, 44, 1039-1049.

Kim, K. T.; Meeuwissen, S. A.; Nolte, R. J.; van Hest, J. C. "Smart nanocontainers and nanoreactors" Nanoscale 2010, 2, 844-858.

(31) Broz, P.; Driamov, S.; Ziegler, J.; Ben-Haim, N.; Marsch, S.; Meier, W.; Hunziker, P. "Toward intelligent nanosize bioreactors: A pH-switchable, channel-equipped, functional polymer nanocontainer" Nano Lett. 2006, 6, 2349-2353. 
(32) Louzao, I.; van Hest, J. C. M. "Permeability Effects on the Efficiency of Antioxidant Nanoreactors" Biomacromolecules 2013, 14, 2364-2372.

Kim, K. T.; Cornelissen, J. J. L. M.; Nolte, R. J. M.; van Hest, J. C. M. "A Polymersome Nanoreactor with Controllable Permeability Induced by Stimuli-Responsive Block Copolymers" Adv. Mater. 2009, 21, 2787-2791.

(34) Tanner, P.; Egli, S.; Balasubramanian, V.; Onaca, O.; Palivan, C. G.; Meier, W. "Can polymeric vesicles that confine enzymatic reactions act as simplified organelles?" FEBS Lett. 2011, 585, 1699-1706.

(35) Peters, R. J. R. W.; Louzao, I.; van Hest, J. C. M. "From polymeric nanoreactors to artificial organelles" Chem. Sci. 2012, 3, 335-342.

(36) Kamat, N. P.; Katz, J. S.; Hammer, D. A. "Engineering Polymersome Protocells" J. Phys. Chem. Lett. 2011, 2, 1612-1623. van Dongen, S. F. M.; de Hoog, H.-P. M.; Peters, R. J. R. W.; Nallani, M.; Nolte, R. J. M.; van Hest, J. C. M. "Biohybrid Polymer Capsules" Chem. Rev. 2009, 109, 6212-6274.

(39) Sukhorukov, G.; Fery, A.; Möhwald, H. "Intelligent micro-and nanocapsules" Prog. Polym. Sci. 2005, 30, 885-897.

(40) Tong, W.; Song, X.; Gao, C. "Layer-by-layer assembly of microcapsules and their biomedical applications" Chem. Soc. Rev. 2012, 41, 6103-6124.

(41) Cui, J.; van Koeverden, M. P.; Muellner, M.; Kempe, K.; Caruso, F. "Emerging methods for the fabrication of polymer capsules" Adv. Colloid Interface Sci. 2014, 207, 14-31.

(42) del Mercato, L. L.; Ferraro, M. M.; Baldassarre, F.; Mancarella, S.; Greco, V.; Rinaldi, R.; 
Leporatti, S. "Biological applications of LbL multilayer capsules: From drug delivery to sensing" Adv. Colloid Interface Sci. 2014, 207, 139-154.

Liu, P. "Stabilization of layer-by-layer engineered multilayered hollow microspheres" Adv. Colloid Interface Sci. 2014, 207, 178-188.

Parakhonskiy, B. V.; Yashchenok, A. M.; Konrad, M.; Skirtach, A. G. "Colloidal micro- and nano-particles as templates for polyelectrolyte multilayer capsules" Adv. Colloid Interface Sci. 2014, 207, 253-264.

Ueda, M.; Uesaka, A.; Kimura, S. "Selective disruption of each part of Janus molecular assemblies by lateral diffusion of stimuli-responsive amphiphilic peptides" Chem. Comm. 2015, 51, 1601-1604.

(46) Blanazs, A.; Ryan, A. J.; Armes, S. P. "Predictive Phase Diagrams for RAFT Aqueous Dispersion Polymerization: Effect of Block Copolymer Composition, Molecular Weight, and Copolymer Concentration" Macromolecules 2012, 45, 5099-5107.

Spulber, M.; Najer, A.; Winkelbach, K.; Glaied, O.; Waser, M.; Pieles, U.; Meier, W.; Bruns, N. "Photoreaction of a hydroxyalkyphenone with the membrane of polymersomes: a versatile method to generate semipermeable nanoreactors" J. Am. Chem. Soc. 2013, 135, 9204-9212. Lomas, H.; Canton, I.; MacNeil, S.; Du, J.; Armes, S. P.; Ryan, A. J.; Lewis, A. L.; Battaglia, G. "Biomimetic pH sensitive polymersomes for efficient DNA encapsulation and delivery" Adv. Mater. 2007, 19, 4238-4246. Battaglia, G.; Ryan, A. J. "The evolution of vesicles from bulk lamellar gels" Nat Mater 2005, 4, 869-876.

(50) Semsarilar, M.; Penfold, N. J. W.; Jones, E. R.; Armes, S. P. "Semi-crystalline diblock 
copolymer nano-objects prepared via RAFT alcoholic dispersion polymerization of stearyl methacrylate" Polym. Chem. 2015, 6, 1751-1757.

(51) Gonzato, C.; Semsarilar, M.; Jones, E. R.; Li, F.; Krooshof, G. J. P.; Wyman, P.; Mykhaylyk, O. O.; Tuinier, R.; Armes, S. P. "Rational Synthesis of Low-Polydispersity Block Copolymer Vesicles in Concentrated Solution via Polymerization-Induced Self-Assembly" J. Am. Chem. Soc. 2014, 136, 11100-11106.

Pei, Y.; Lowe, A. B. "Polymerization-induced self-assembly: ethanolic RAFT dispersion polymerization of 2-phenylethyl methacrylate" Polym. Chem. 2014, 5, 2342-2351.

(53) Battaglia, G.; Tomas, S.; Ryan, A. J. "Lamellarsomes: metastable polymeric multilamellar aggregates" Soft Matter 2007, 3, 470-475.

Smart, T.; Lomas, H.; Massignani, M.; Flores-Merino, M. V.; Perez, L. R.; Battaglia, G. "Block copolymer nanostructures" Nano Today 2008, 3, 38-46.

Robertson, J. D.; Yealland, G.; Avila-Olias, M.; Chierico, L.; Bandmann, O.; Renshaw, S. A.; Battaglia, G. "pH-Sensitive Tubular Polymersomes: Formation and Applications in Cellular Delivery" ACS Nano 2014, 8, 4650-4661.

(56) Adams, D. J.; Butler, M. F.; Weaver, A. C. "Effect of Block Length, Polydispersity, and Salt Concentration on PEO-PDEAMA Block Copolymer Structures in Dilute Solution" Langmuir 2006, $22,4534-4540$.

(57) Adams, D. J.; Adams, S.; Atkins, D.; Butler, M. F.; Furzeland, S. "Impact of mechanism of formation on encapsulation in block copolymer vesicles" $J$ Control Release 2008, 128, $165-170$.

(58) Onaca, O.; Enea, R.; Hughes, D. W.; Meier, W. "Stimuli-Responsive Polymersomes as 126 
Nanocarriers for Drug and Gene Delivery" Macromol. Biosci. 2009, 9, 129-139.

(59) Pegoraro, C.; MacNeil, S.; Battaglia, G. "Transdermal drug delivery: from micro to nano" Nanoscale 2012, 4, 1881-1894.

(60) Liao, J. F.; Wang, C.; Wang, Y. J.; Luo, F.; Qian, Z. Y. "Recent Advances in Formation, Properties, and Applications of Polymersomes" Curr. Pharm. Design 2012, 18, 3432-3441.

(61) Tong, R.; Lu, X. L.; Xia, H. S. "A facile mechanophore functionalization of an amphiphilic block copolymer towards remote ultrasound and redox dual stimulus responsiveness" Chem. Comm. 2014, 50, 3575-3578.

(62) Howse, J. R.; Jones, R. A. L.; Battaglia, G.; Ducker, R. E.; Leggett, G. J.; Ryan, A. J. "Templated formation of giant polymer vesicles with controlled size distributions" Nat Mater 2009, $8,507-511$.

(63) Angelova, M.; Dimitrov, D. "Liposome electroformation" Faraday Discuss. Chem. Soc. 1986, $81,303-311$.

(64) Wesolowska, O.; Michalak, K.; Maniewska, J.; Hendrich, A. B. "Giant unilamellar vesicles - a perfect tool to visualize phase separation and lipid rafts in model systems" Acta Biochim. Pol. 2009, 56, 33-39.

Le Meins, J. F.; Carlsen, A. C., A.; Glaser, N.; Lecommandoux, S. "Block Copolymer Vesicle Permeability Measured by Osmotic Swelling and Shrinking" Langmuir 2011, 27, 4884-4890.

(66) Gaitzsch, J.; Appelhans, D.; Gräfe, D.; Schwille, P.; Voit, B. "Photo-crosslinked and pH sensitive polymersomes for triggering the loading and release of cargo" Chem. Comm. 2011, $47,3466-3468$.

(67) Meeuwissen, S. A.; Kim, K. T.; Chen, Y.; Pochan, D. J.; van Hest, J. C. M. "Controlled Shape 127 
Transformation of Polymersome Stomatocytes" Angew. Chem. Int. Ed. 2011, 50, 7070-7073.

(68) Fernyhough, C.; Ryan, A. J.; Battaglia, G. "pH controlled assembly of a polybutadiene-poly(methacrylic acid) copolymer in water: packing considerations and kinetic limitations" Soft Matter 2009, 5, 1674-1682.

Themistou, E.; Battaglia, G.; Armes, S. P. "Facile synthesis of thiol-functionalized amphiphilic polylactide-methacrylic diblock copolymers" Polym. Chem. 2014, 5, 1405-1417.

(70) Liu, K. K.; Wan, K. T. "Mechanics of biomimetic/biological vesicles" Mater. Sci. Found. 2008, 41-43, 115-133.

(71) Canton, I.; Battaglia, G. "Endocytosis at the nanoscale" Chem. Soc. Rev. 2012, 41, 2718-2739.

(72) Hong, C. Y.; You, Y. Z.; Pan, C. Y. "Synthesis and characterization of well-defined diblock and triblock copolymers of poly(N-isopropylacrylamide) and poly(ethylene oxide)" J. Polym. Sci. A Polym. Chem 2004, 42, 4873-4881.

(73) Chen, X. R.; Ding, X. B.; Zheng, Z. H.; Peng, Y. X. "A self-assembly approach to temperature-responsive polymer nanocontainers" Macromol. Rapid Commun. 2004, 25, 1575-1578.

(74) Park, W. M.; Champion, J. A. "Thermally Triggered Self-Assembly of Folded Proteins into Vesicles" J. Am. Chem. Soc. 2014, 136, 17906-17909.

(75) Luo, T. Z.; He, L. R.; Theato, P.; Kiick, K. L. "Thermoresponsive Self-Assembly of Nanostructures from a Collagen-Like Peptide-Containing Diblock Copolymer" Macromol. Biosci. 2015, 15, 111-123.

(76) Yassin, M.; Appelhans, D.; Mendes, R.; Rümmeli, M.; Voit, B. "pH-dependent Release of Doxorubicin from Fast Photo Cross-linkable Polymersomes Based on Benzophenone Units" 
Chem. Eur. J. 2012, 18, 12227-12231.

Wei, J.; Ju, X.-J.; Zou, X.-Y.; Xie, R.; Wang, W.; Liu, Y.-M.; Chu, L.-Y. "Multi-Stimuli-Responsive Microcapsules for Adjustable Controlled-Release" Adv. Funct. Mater. 2014, 24, 3312-3323.

Kim, I.; Lee, N. E.; Jeong, Y. J.; Chung, Y. H.; Cho, B. K.; Lee, E. "Micellar and vesicular nanoassemblies of triazole-based amphiphilic probes triggered by mercury(II) ions in a $100 \%$ aqueous medium" Chem. Comm. 2014, 50, 14006-14009.

Anraku, Y.; Kishimura, A.; Oba, M.; Yamasaki, Y.; Kataoka, K. "Spontaneous Formation of Nanosized Unilamellar Polyion Complex Vesicles with Tunable Size and Properties" J Am Chem Soc 2010, 132, 1631-1636.

(80) Schrage, S.; Sigel, R.; Schlaad, H. "Formation of amphiphilic polyion complex vesicles from mixtures of oppositely charged block ionomers" Macromolecules 2003, 36, 1417-1420.

Dong, W. F.; Kishimura, A.; Anraku, Y.; Chuanoi, S.; Kataoka, K. "Monodispersed Polymeric Nanocapsules: Spontaneous Evolution and Morphology Transition from Reducible Hetero-PEG PICmicelles by Controlled Degradation" J Am Chem Soc 2009, 131, 3804-+.

(82) Anraku, Y.; Kishimura, A.; Yamasaki, Y.; Kataoka, K. "Living Unimodal Growth of Polyion Complex Vesicles via Two-Dimensional Supramolecular Polymerization" J Am Chem Soc 2013, 135, 1423-1429.

Noireaux, V.; Libchaber, A. "A vesicle bioreactor as a step toward an artificial cell assembly" Proc. Natl. Acad. Sci. U.S.A 2004, 101, 17669-17674.

Marguet, M.; Sandre, O.; Lecommandoux, S. "Polymersomes in "Gelly" Polymersomes: Toward Structural Cell Mimicry" Langmuir 2011, 28, 2035-2043. 
Marguet, M.; Edembe, L.; Lecommandoux, S. "Polymersomes in polymersomes: multiple loading and permeability control" Angew. Chem. Int. Ed. 2012, 51, 1173-1176.

(86)

Peters, R. J. R. W.; Marguet, M.; Marais, S.; Fraaije, M. W.; van Hest, J. C. M.; Lecommandoux, S. "Cascade Reactions in Multicompartmentalized Polymersomes" Angew. Chem. Int. Ed. 2014, 53, 146-150.

(87) Teh, S. Y.; Lin, R.; Hung, L. H.; Lee, A. P. "Droplet microfluidics" Lab on a Chip 2008, 8, 198-220.

(88) Brown, L.; McArthur, S. L.; Wright, P. C.; Lewis, A.; Battaglia, G. "Polymersome production on a microfluidic platform using $\mathrm{pH}$ sensitive block copolymers" Lab on a Chip 2010, 10, 1922-1928.

(89) Thiele, J.; Abate, A. R.; Shum, H. C.; Bachtler, S.; Forster, S.; Weitz, D. A. "Fabrication of Polymersomes using Double-Emulsion Templates in Glass-Coated Stamped Microfluidic Devices" Small 2010, 6, 1723-1727.

(90) Kim, S. H.; Kim, J. W.; Kim, D. H.; Han, S. H.; Weitz, D. A. "Polymersomes Containing a Hydrogel Network for High Stability and Controlled Release" Small 2013, 9, 124-131.

(91) Kim, S. H.; Nam, J.; Kim, J. W.; Kim, D. H.; Han, S. H.; Weitz, D. A. "Formation of polymersomes with double bilayers templated by quadruple emulsions" Lab on a Chip 2013, $13,1351-1356$.

(92) Kim, S. H.; Kim, J. W.; Kim, D. H.; Han, S. H.; Weitz, D. A. "Enhanced-throughput production of polymersomes using a parallelized capillary microfluidic device" Microfluidics and Nanofluidics 2013, 14, 509-514.

(93) Kim, S.-H.; Shum, H. C.; Kim, J. W.; Cho, J.-C.; Weitz, D. A. "Multiple Polymersomes for 
Programmed Release of Multiple Components" J. Am. Chem. Soc. 2011, 133, 15165-15171.

(94) Sugihara, S.; Blanazs, A.; Armes, S. P.; Ryan, A. J.; Lewis, A. L. "Aqueous Dispersion Polymerization: A New Paradigm for in Situ Block Copolymer Self-Assembly in Concentrated Solution" J. Am. Chem. Soc. 2011, 133, 15707-15713.

Warren, N. J.; Mykhaylyk, O. O.; Mahmood, D.; Ryan, A. J.; Armes, S. P. "RAFT Aqueous

Dispersion Polymerization Yields Poly(ethylene glycol)-Based Diblock Copolymer Nano-Objects with Predictable Single Phase Morphologies" J. Am. Chem. Soc. 2014, 136, 1023-1033.

(96) Ratcliffe, L. P. D.; Blanazs, A.; Williams, C. N.; Brown, S. L.; Armes, S. P. "RAFT polymerization of hydroxy-functional methacrylic monomers under heterogeneous conditions: effect of varying the core-forming block" Polym. Chem. 2014, 5, 3643-3655.

(97) Chambon, P.; Blanazs, A.; Battaglia, G.; Armes, S. P. "Facile Synthesis of Methacrylic ABC Triblock Copolymer Vesicles by RAFT Aqueous Dispersion Polymerization" Macromolecules 2012, 45, 5081-5090.

(98) Warren, N. J.; Mykhaylyk, O. O.; Ryan, A. J.; Williams, M.; Doussineau, T.; Dugourd, P.; Antoine, R.; Portale, G.; Armes, S. P. "Testing the Vesicular Morphology to Destruction: Birth and Death of Diblock Copolymer Vesicles Prepared via Polymerization-Induced Self-Assembly" J. Am. Chem. Soc. 2015, 137, 1929-1937.

(99) Agut, W.; Brûlet, A.; Schatz, C.; Taton, D.; Lecommandoux, S. b. "pH and Temperature Responsive Polymeric Micelles and Polymersomes by Self-Assembly of Poly[2-(dimethylamino)ethyl methacrylate]-b-Poly(glutamic acid) Double Hydrophilic Block Copolymers" Langmuir 2010, 26, 10546-10554. 
(100) Liu, B. W.; Zhou, H.; Zhou, S. T.; Zhang, H. J.; Feng, A. C.; Jian, C. M.; Hu, J.; Gao, W. P.; Yuan, J. Y. "Synthesis and Self-Assembly of CO2-Temperature Dual Stimuli-Responsive Triblock Copolymers" Macromolecules 2014, 47, 2938-2946.

(101) Guo, W. J.; Wang, T. S.; Tang, X. D.; Zhang, Q.; Yu, F. Q.; Pei, M. S. "Triple Stimuli-Responsive Amphiphilic Glycopolymer" J. Polym. Sci. A Polym. Chem 2014, 52, 2131-2138

(102) Fielding, L. A.; Lane, J. A.; Derry, M. J.; Mykhaylyk, O. O.; Armes, S. P. "Thermo-responsive Diblock Copolymer Worm Gels in Non-polar Solvents" J. Am. Chem. Soc. 2014, 136, 5790-5798.

(103) Blomberg, S.; Ostberg, S.; Harth, E.; Bosman, A. W.; Van Horn, B.; Hawker, C. J. "Production of crosslinked, hollow nanoparticles by surface - initiated living free - radical polymerization" J. Polym. Sci. A Polym. Chem 2002, 40, 1309-1320.

(104) Morinaga, T.; Ohkura, M.; Ohno, K.; Tsujii, Y.; Fukuda, T. "Monodisperse silica particles grafted with concentrated oxetane-carrying polymer brushes: Their synthesis by surface-initiated atom transfer radical polymerization and use for fabrication of hollow spheres" Macromolecules 2007, 40, 1159-1164.

(105) Fu, G.; Shang, Z.; LIANG, H.; Kang, E.; Neoh, K. "Preparation of cross-linked polystyrene hollow nanospheres via surface-initiated atom transfer radical polymerizations" Macromolecules 2005, 38, 7867-7871.

(106) Matyjaszewski, K.; Tsarevsky, N. V. "Nanostructured functional materials prepared by atom transfer radical polymerization" Nat. Chem. 2009, 1, 276-288.

(107) Boyer, C.; Bulmus, V.; Davis, T. R.; Ladmiral, V.; Liu, J.; Perrier, S. "Bioapplications of 132 
RAFT polymerization" Chem. Rev. 2009, 109, 5402-5436.

(108) Xu, X.; Asher, S. A. "Synthesis and utilization of monodisperse hollow polymeric particles in photonic crystals" J. Am. Chem. Soc. 2004, 126, 7940-7945.

(109) Li, G. L.; Xu, L. Q.; Tang, X.; Neoh, K.; Kang, E. "Hairy Hollow Microspheres of Fluorescent Shell and Temperature-Responsive Brushes via Combined Distillation-Precipitation Polymerization and Thiol- ene Click Chemistry" Macromolecules 2010, 43, 5797-5803.

(110) Huang, X.; Appelhans, D.; Formanek, P.; Simon, F.; Voit, B. "Synthesis of Well-Defined Photo-Cross-Linked Polymeric Nanocapsules by Surface-Initiated RAFT Polymerization" Macromolecules 2011, 44, 8351-8360.

(111) Huang, X.; Appelhans, D.; Formanek, P.; Simon, F.; Voit, B. "Tailored Synthesis of Intelligent Polymer Nanocapsules: An Investigation of Controlled Permeability and pH-Dependent Degradability" ACS Nano 2012, 6, 9718-9726.

(112) Zhou, K.; Tong, L.; Deng, J.; Yang, W. "Hollow polymeric microspheres grafted with optically active helical polymer chains: Preparation and their chiral recognition ability" J Mater Chem 2010, 20, 781-789.

(113) Chen, F.; Jiang, X.; Liu, R.; Yin, J. "Polymeric vesicles with well-defined poly(methyl methacrylate) (PMMA) brushes via surface-initiated photopolymerization (SIPP)" Polym. Chem. 2011, 2, 614-618.

(114) Boyer, C.; Whittaker, M. R.; Nouvel, C.; Davis, T. P. "Synthesis of hollow polymer nanocapsules exploiting gold nanoparticles as sacrificial templates" Macromolecules 2010, 43, 1792-1799.

(115) Lowe, A. B.; Sumerlin, B. S.; Donovan, M. S.; McCormick, C. L. "Facile Preparation of 
Transition Metal Nanoparticles Stabilized by Well-Defined (Co)polymers Synthesized via Aqueous Reversible Addition-Fragmentation Chain Transfer Polymerization†" J. Am. Chem. Soc. 2002, 124, 11562-11563.

(116) Roth, P. J.; Theato, P. "Versatile Synthesis of Functional Gold Nanoparticles: Grafting Polymers From and Onto" Chem. Mater. 2008, 20, 1614-1621.

(117) Hotz, J.; Meier, W. "Vesicle-templated polymer hollow spheres" Langmuir 1998, 14, 1031-1036.

(118) Ali, S. I.; Heuts, J. P. A.; van Herk, A. M. "Vesicle-templated pH-responsive polymeric nanocapsules" Soft Matter 2011, 7, 5382-5390.

(119) Ali, S. I.; Heuts, J. P. A.; van Herk, A. M. "Controlled Synthesis of Polymeric Nanocapsules by RAFT-Based Vesicle Templating" Langmuir 2010, 26, 7848-7858.

(120) Musyanovych, A.; Landfester, K. "Polymer Micro- and Nanocapsules as Biological Carriers with Multifunctional Properties" Macromol. Biosci. 2014, 14, 458-477.

(121) Landfester, K.; Musyanovych, A.; Mailänder, V. "From polymeric particles to multifunctional nanocapsules for biomedical applications using the miniemulsion process" J. Polym. Sci. A Polym. Chem 2010, 48, 493-515.

(122) Tiarks, F.; Landfester, K.; Antonietti, M. "Preparation of polymeric nanocapsules by miniemulsion polymerization" Langmuir 2001, 17, 908-918.

(123) Musyanovych, A.; Landfester, K. "Synthesis of poly (butylcyanoacrylate) nanocapsules by interfacial polymerization in miniemulsions for the delivery of DNA molecules" Prog. Colloid Polym. Sci. 2008, 120-127.

(124) Jagielski, N.; Sharma, S.; Hombach, V.; Mailänder, V.; Rasche, V.; Landfester, K. 
"Nanocapsules synthesized by miniemulsion technique for application as new contrast agent materials" Macromol. Chem. Phys. 2007, 208, 2229-2241.

(125) Crespy, D.; Stark, M.; Hoffmann-Richter, C.; Ziener, U.; Landfester, K. "Polymeric nanoreactors for hydrophilic reagents synthesized by interfacial polycondensation on miniemulsion droplets" Macromolecules 2007, 40, 3122-3135.

(126) Torini, L.; Argillier, J. F.; Zydowicz, N. "Interfacial Polycondensation Encapsulation in Miniemulsion" Macromolecules 2005, 38, 3225-3236.

(127) Luo, Y.; Zhou, X. "Nanoencapsulation of a hydrophobic compound by a miniemulsion polymerization process" J. Polym. Sci. A Polym. Chem 2004, 42, 2145-2154.

(128) Cao, Z.; Shan, G. "Synthesis of polymeric nanocapsules with a crosslinked shell through interfacial miniemulsion polymerization" J. Polym. Sci. A Polym. Chem 2009, 47, 1522-1534.

(129) Theisinger, S.; Schoeller, K.; Osborn, B.; Sarkar, M.; Landfester, K. "Encapsulation of a Fragrance via Miniemulsion Polymerization for Temperature-Controlled Release" Macromol. Chem. Phys. 2009, 210, 411-420.

(130) Romio, A. P.; Sayer, C.; Araújo, P. H. H.; Al-Haydari, M.; Wu, L.; da Rocha, S. R. P. "Nanocapsules by Miniemulsion Polymerization with Biodegradable Surfactant and Hydrophobe" Macromol. Chem. Phys. 2009, 210, 747-751.

(131) Zetterlund, P. B.; Saka, Y.; Okubo, M. "Gelation and Hollow Particle Formation in Nitroxide-Mediated Radical Copolymerization of Styrene and Divinylbenzene in Miniemulsion" Macromol. Chem. Phys. 2009, 210, 140-149.

(132) Utama, R. H.; Drechsler, M.; Förster, S.; Zetterlund, P. B.; Stenzel, M. H. "Synthesis of pH-Responsive Nanocapsules via Inverse Miniemulsion Periphery RAFT Polymerization and 
Post-Polymerization Reaction" ACS Macro Lett. 2014, 3, 935-939.

(133) Utama, R. H.; Stenzel, M. H.; Zetterlund, P. B. "Inverse Miniemulsion Periphery RAFT Polymerization: A Convenient Route to Hollow Polymeric Nanoparticles with an Aqueous Core" Macromolecules 2013, 46, 2118-2127.

(134) Zhang, Y.; Zhu, S.; Yin, L.; Qian, F.; Tang, C.; Yin, C. "Preparation, characterization and biocompatibility of poly(ethylene glycol)-poly(n-butyl cyanoacrylate) nanocapsules with oil core via miniemulsion polymerization" Eur. Polym. J. 2008, 44, 1654-1661.

(135) Baier, G.; Friedemann, K.; Leuschner, E.-M.; Musyanovych, A.; Landfester, K. "pH Stability of Poly(urethane/urea) Capsules Synthesized from Different Hydrophilic Monomers via Interfacial Polyaddition in the Inverse Miniemulsion Process" Macromol. Symp. 2013, $331-332,71-80$.

(136) Rosenbauer, E.-M.; Landfester, K.; Musyanovych, A. "Surface-Active Monomer as a Stabilizer for Polyurea Nanocapsules Synthesized via Interfacial Polyaddition in Inverse Miniemulsion" Langmuir 2009, 25, 12084-12091.

(137) van Zyl, A. J. P.; Bosch, R. F. P.; McLeary, J. B.; Sanderson, R. D.; Klumperman, B. "Synthesis of styrene based liquid-filled polymeric nanocapsules by the use of RAFT-mediated polymerization in miniemulsion" Polymer 2005, 46, 3607-3615.

(138) Lu, F.; Luo, Y.; Li, B. "A Facile Route to Synthesize Highly Uniform Nanocapsules: Use of Amphiphilic Poly(acrylic acid)-block-polystyrene RAFT Agents to Interfacially Confine Miniemulsion Polymerization" Macromol. Rapid Commun. 2007, 28, 868-874.

(139) Luo, Y.; Gu, H. "A General Strategy for Nano-Encapsulation via Interfacially Confined Living/Controlled Radical Miniemulsion Polymerization" Macromol. Rapid Commun. 2006, 
$27,21-25$.

(140) Luo, Y.; Gu, H. "Nanoencapsulation via interfacially confined reversible addition fragmentation transfer (RAFT) miniemulsion polymerization" Polymer 2007, 48, 3262-3272.

(141) Li, W.; Matyjaszewski, K.; Albrecht, K.; Möller, M. "Reactive surfactants for polymeric nanocapsules via interfacially confined miniemulsion ATRP" Macromolecules 2009, 42, $8228-8233$.

(142) Huang, C.-Y.; Chen, C.-M.; Lee, Y.-D. "Synthesis of high loading and encapsulation efficient paclitaxel-loaded poly(n-butyl cyanoacrylate) nanoparticles via miniemulsion" Int. J. Pharm. 2007, 338, 267-275.

(143) Fresta, M.; Cavallaro, G.; Giammona, G.; Wehrli, E.; Puglisi, G. "Preparation and characterization of polyethyl-2-cyanoacrylate nanocapsules containing antiepileptic drugs" Biomaterials 1996, 17, 751-758.

(144) Aboubakar, M.; Puisieux, F.; Couvreur, P.; Vauthier, C. "Physico-chemical characterization of insulin-loaded poly(isobutylcyanoacrylate) nanocapsules obtained by interfacial polymerization" Int. J. Pharm. 1999, 183, 63-66.

(145) Baier, G.; Musyanovych, A.; Dass, M.; Theisinger, S.; Landfester, K. "Cross-Linked Starch Capsules Containing dsDNA Prepared in Inverse Miniemulsion as "Nanoreactors" for Polymerase Chain Reaction" Biomacromolecules 2010, 11, 960-968.

(146) Baier, G.; Baumann, D.; Siebert, J. M.; Musyanovych, A.; Mailänder, V.; Landfester, K. "Suppressing Unspecific Cell Uptake for Targeted Delivery Using Hydroxyethyl Starch Nanocapsules" Biomacromolecules 2012, 13, 2704-2715.

(147) Freichels, H.; Wagner, M.; Okwieka, P.; Meyer, R. G.; Mailander, V.; Landfester, K.; 
Musyanovych, A. "(Oligo)mannose functionalized hydroxyethyl starch nanocapsules: en route to drug delivery systems with targeting properties" J. Mater. Chem. B 2013, 1, 4338-4348.

(148) Paiphansiri, U.; Dausend, J.; Musyanovych, A.; Mailänder, V.; Landfester, K. "Fluorescent Polyurethane Nanocapsules Prepared via Inverse Miniemulsion: Surface Functionalization for Use as Biocarriers" Macromol. Biosci. 2009, 9, 575-584.

(149) Paiphansiri, U.; Tangboriboonrat, P.; Landfester, K. "Antiseptic Nanocapsule Formation via Controlling Polymer Deposition onto Water-in-Oil Miniemulsion Droplets" Macromol. Symp. 2007, 251, 54-62.

(150) Paiphansiri, U.; Tangboriboonrat, P.; Landfester, K. "Polymeric Nanocapsules Containing an Antiseptic Agent Obtained by Controlled Nanoprecipitation onto Water-in-Oil Miniemulsion Droplets" Macromol. Biosci. 2006, 6, 33-40.

(151) Huang, X.; Li, M.; Mann, S. "Membrane-mediated cascade reactions by enzyme-polymer proteinosomes" Chem. Comm. 2014, 50, 6278-6280.

(152) Huang, X.; Patil, A. J.; Li, M.; Mann, S. "Design and construction of higher-order structure and function in proteinosome-based protocells" J. Am. Chem. Soc. 2014, 136, 9225-9234.

(153) Kumar, C. V.; Chaudhari, A. "Proteins immobilized at the galleries of layered alpha-zirconium phosphate: Structure and activity studies" J. Am. Chem. Soc. 2000, 122, 830-837.

(154) Graff, A.; Winterhalter, M.; Meier, W. "Nanoreactors from Polymer-Stabilized Liposomes" Langmuir 2001, 17, 919-923.

(155) Gunkel-Grabole, G.; Sigg, S.; Lomora, M.; Lorcher, S.; Palivan, C. G.; Meier, W. P. "Polymeric 3D nano-architectures for transport and delivery of therapeutically relevant biomacromolecules" Biomater. Sci. 2015, 3, 25-40. 
(156) Kumar, M.; Habel, J. E. O.; Shen, Y. X.; Meier, W. P.; Walz, T. "High-Density Reconstitution of Functional Water Channels into Vesicular and Planar Block Copolymer Membranes" J. Am. Chem. Soc. 2012, 134, 18631-18637.

(157) Kumar, M.; Grzelakowski, M.; Zilles, J.; Clark, M.; Meier, W. "Highly permeable polymeric membranes based on the incorporation of the functional water channel protein Aquaporin Z" Proc. Natl. Acad. Sci. U.S.A 2007, 104, 20719-20724.

(158) Tanner, P.; Balasubramanian, V.; Palivan, C. G. "Aiding Nature's Organelles: Artificial Peroxisomes Play Their Role" Nano Lett. 2013, 13, 2875-2883.

(159) Graff, A.; Sauer, M.; Van Gelder, P.; Meier, W. "Virus-assisted loading of polymer nanocontainer" Proc. Natl. Acad. Sci. U.S.A 2002, 99, 5064-5068.

(160) Choi, H. J.; Montemagno, C. D. "Artificial organelle: ATP synthesis from cellular mimetic polymersomes" Nano Lett. 2005, 5, 2538-2542.

(161) Zhang, X. Y.; Fu, W. Y.; Palivan, C. G.; Meier, W. "Natural channel protein inserts and functions in a completely artificial, solid-supported bilayer membrane" Scientific Reports $2013,3,7$.

(162) Stoenescu, R.; Meier, W. "Vesicles with asymmetric membranes from amphiphilic ABC triblock copolymers" Chem. Comm. 2002, 3016-3017.

(163) Stoenescu, R.; Graff, A.; Meier, W. "Asymmetric ABC-triblock copolymer membranes induce a directed insertion of membrane proteins" Macromol. Biosci. 2004, 4, 930-935.

(164) Ranquin, A.; Versees, W.; Meier, W.; Steyaert, J.; Van Gelder, P. "Therapeutic Nanoreactors: Combining Chemistry and Biology in a Novel Triblock Copolymer Drug Delivery System" Nano Lett. 2005, 5, 2220-2224. 
(165) Choi, H. J.; Brooks, E.; Montemagno, C. D. "Synthesis and characterization of nanoscale biomimetic polymer vesicles and polymer membranes for bioelectronic applications" Nanotechnology 2005, 16, S143-S149.

(166) Tanner, P.; Onaca, O.; Balasubramanian, V.; Meier, W.; Palivan, C. G. "Enzymatic Cascade Reactions inside Polymeric Nanocontainers: A Means to Combat Oxidative Stress" Chem. Eur. J. 2011, 17, 4552-4560.

(167) Balasubramanian, V.; Onaca, O.; Ezhevskaya, M.; Van Doorslaer, S.; Sivasankaran, B.; Palivan, C. G. "A surprising system: polymeric nanoreactors containing a mimic with dual-enzyme activity" Soft Matter 2011, 7, 5595-5603.

(168) De Oliveira, H.; Thevenot, J.; Lecommandoux, S. "Smart polymersomes for therapy and diagnosis: fast progress toward multifunctional biomimetic nanomedicines" Wiley Interdiscip Rev Nanomed Nanobiotechno 2012, 4, 525-546.

(169) Pawar, P. V.; Gohil, S. V.; Jain, J. P.; Kumar, N. "Functionalized polymersomes for biomedical applications" Polym. Chem. 2013, 4, 3160-3176.

(170) Canton, I.; Massignani, M.; Patikarnmonthon, N.; Chierico, L.; Robertson, J.; Renshaw, S. A.; Warren, N. J.; Madsen, J. P.; Armes, S. P.; Lewis, A. L.; Battaglia, G. "Fully synthetic polymer vesicles for intracellular delivery of antibodies in live cells" Faseb J. 2013, 27, 98-108.

(171) Akinc, A.; Battaglia, G. "Exploiting Endocytosis for Nanomedicines" Cold Spring Harb Perspect Biol. 2013, 5, DOI: doi:10.1101/cshperspect.a016980.

(172) Lomas, H.; Massignani, M.; Abdullah, K. A.; Canton, I.; Lo Presti, C.; MacNeil, S.; Du, J. Z.; Blanazs, A.; Madsen, J.; Armes, S. P.; Lewis, A. L.; Battaglia, G. "Non-cytotoxic polymer vesicles for rapid and efficient intracellular delivery" Faraday Discuss 2008, 139, 143-159. 
(173) Itel, F.; Al-Samir, S.; Oberg, F.; Chami, M.; Kumar, M.; Supuran, C. T.; Deen, P. M. T.; Meier, W.; Hedfalk, K.; Gros, G.; Endeward, V. "CO2 permeability of cell membranes is regulated by membrane cholesterol and protein gas channels" Faseb J. 2012, 26, 5182-5191.

(174) Yan, Q.; Wang, J.; Yin, Y.; Yuan, J. "Breathing Polymersomes: CO2 - Tuning Membrane Permeability for Size - Selective Release, Separation, and Reaction" Angew. Chem. Int. Ed. 2013, 52, 5070-5073.

(175) Nuyken, O.; Pask, S. D. "Ring-Opening Polymerization-An Introductory Review" Polymers 2013, 5, 361-403.

(176) Lee, E. S.; Shin, H. J.; Na, K.; Bae, Y. H. "Poly(L-histidine)-PEG block copolymer micelles and pH-induced destabilization" J Control Release 2003, 90, 363-374.

(177) Mavrogiorgis, D.; Bilalis, P.; Karatzas, A.; Skoulas, D.; Fotinogiannopoulou, G.; Iatrou, H. "Controlled polymerization of histidine and synthesis of well-defined stimuli responsive polymers. Elucidation of the structure-aggregation relationship of this highly multifunctional material" Polym. Chem. 2014, 5, 6256-6278.

(178) Li, Z.; Qiu, L. P.; Chen, Q.; Hao, T. N.; Qiao, M. X.; Zhao, H. X.; Zhang, J.; Hu, H. Y.; Zhao, X. L.; Chen, D. W.; Mei, L. "pH-sensitive nanoparticles of poly(L-histidine)-poly(lactide-co-glycolide)-tocopheryl polyethylene glycol succinate for anti-tumor drug delivery" Acta Biomater 2015, 11, 137-150.

(179) LoPresti, C.; Lomas, H.; Massignani, M.; Smart, T.; Battaglia, G. "Polymersomes: nature inspired nanometer sized compartments" J Mater Chem 2009, 19, 3576-3590.

(180) Giacomelli, C.; Le Men, L.; Borsali, R.; Lai-Kee-Him, J.; Brisson, A.; Armes, S. P.; Lewis, A. L. "Phosphorylcholine-based pH-responsive diblock copolymer micelles as drug delivery

141 
vehicles: Light scattering, electron microscopy, and fluorescence experiments" Biomacromolecules 2006, 7, 817-828.

(181) Lomas, H.; Du, J. Z.; Canton, I.; Madsen, J.; Warren, N.; Armes, S. P.; Lewis, A. L.; Battaglia, G. "Efficient Encapsulation of Plasmid DNA in pH-Sensitive PMPC-PDPA Polymersomes: Study of the Effect of PDPA Block Length on Copolymer-DNA Binding Affinity" Macromol. Biosci. 2010, 10, 513-530.

(182) Canton, I.; Battaglia, G. "Polymersomes-mediated delivery of fluorescent probes for targeted and long-term imaging in live cell microscopy" Methods Mol Biol 2013, 991, 343-351.

(183) Colley, H. E.; Hearnden, V.; Avila-Olias, M.; Cecchin, D.; Canton, I.; Madsen, J.; MacNeil, S.; Warren, N.; Hu, K.; McKeating, J. A.; Armes, S. P.; Murdoch, C.; Thornhill, M. H.; Battaglia, G. "Polymersome-Mediated Delivery of Combination Anticancer Therapy to Head and Neck Cancer Cells: 2D and 3D in Vitro Evaluation" Mol Pharmaceut 2014, 11, 1176-1188.

(184) Yu, S. Y.; Azzam, T.; Rouiller, I.; Eisenberg, A. "'Breathing" Vesicles" J. Am. Chem. Soc. 2009, 131, 10557-10566.

(185) Wang, X. Y.; Sun, H. L.; Meng, F. H.; Cheng, R.; Deng, C.; Zhong, Z. Y. "Galactose-Decorated Reduction-Sensitive Degradable Chimaeric Polymersomes as a Multifunctional Nanocarrier To Efficiently Chaperone Apoptotic Proteins into Hepatoma Cells" Biomacromolecules 2013, 14, 2873-2882.

(186) Gaitzsch, J.; Appelhans, D.; Wang, L.; Battaglia, G.; Voit, B. "Synthetic Bio - nanoreactor: Mechanical and Chemical Control of Polymersome Membrane Permeability" Angew. Chem. Int. Ed. 2012, 51, 4448-4451.

(187) Grafe, D.; Gaitzsch, J.; Appelhans, D.; Voit, B. "Cross-linked polymersomes as nanoreactors 142 
for controlled and stabilized single and cascade enzymatic reactions" Nanoscale 2014, 6, 10752-10761.

(188) Hong, L. Z.; Zhang, Z. H.; Zhang, Y.; Zhang, W. A. "Synthesis and Self-Assembly of Stimuli-Responsive Amphiphilic Block Copolymers Based on Polyhedral Oligomeric Silsesquioxane" J. Polym. Sci. A Polym. Chem 2014, 52, 2669-2683.

(189) Yassin, M. A.; Appelhans, D.; Wiedemuth, R.; Formanek, P.; Boye, S.; Lederer, A.; Temme, A.; Voit, B. "Overcoming Concealment Effects of Targeting Moieties in the PEG Corona: Controlled Permeable Polymersomes Decorated with Folate-Antennae for Selective Targeting of Tumor Cells" Small 2015, 11, 1580-1591.

(190) Liu, H. B.; Guo, Z. R.; He, S.; Yin, H. Y.; Fei, C. H.; Feng, Y. J. "CO2-driven vesicle to micelle regulation of amphiphilic copolymer: random versus block strategy" Polym. Chem. 2014, 5, 4756-4763.

(191) Lin, F.; Wu, J. L.; Liu, J. G. "The study of far UV irradiation of 2-methoxycytosine in phosphate solution and its novel photoproduct" J. Photochem. Photobiol. A-Chem. 2000, 131, 49-53.

(192) Amos, R. C.; Nazemi, A.; Bonduelle, C. V.; Gillies, E. R. "Tuning polymersome surfaces: functionalization with dendritic groups" Soft Matter 2012, 8, 5947-5958.

(193) Tai, W. Y.; Mo, R.; Di, J.; Subramanian, V.; Gu, X.; Buse, J. B.; Gu, Z. "Bio-lnspired Synthetic Nanovesicles for Glucose-Responsive Release of Insulin" Biomacromolecules 2014, 15, 3495-3502.

(194) Chen, X. R.; Ding, X. B.; Zheng, Z. H.; Peng, Y. X. "Thermosensitive cross-linked polymer vesicles for controlled release system" New Journal of Chemistry 2006, 30, 577-582.

143 
(195) Miao, K.; Liu, H. H.; Zhao, Y. L. "Thermo, pH and reduction responsive coaggregates comprising $\mathrm{AB}(2) \mathrm{C}(2)$ star terpolymers for multi-triggered release of doxorubicin" Polym.

Chem. 2014, 5, 3335-3345.

(196) Hocine, S.; Cui, D.; Rager, M. N.; Di Cicco, A.; Liu, J. M.; Wdzieczak-Bakala, J.; Brulet, A.; Li, M. H. "Polymersomes with PEG Corona: Structural Changes and Controlled Release Induced by Temperature Variation" Langmuir 2013, 29, 1356-1369.

(197) Lu, C. L.; Urban, M. W. "Tri-Phasic Size- and Janus Balance-Tunable Colloidal Nanoparticles (JNPs)" Acs Macro Lett. 2014, 3, 346-352.

(198) Liang, X.; Kozlovskaya, V.; Cox, C. P.; Wang, Y.; Saeed, M.; Kharlampieva, E. "Synthesis and Self-Assembly of Thermosensitive Double-Hydrophilic Poly(N-vinylcaprolactam)-b-poly(N-vinyl-2-pyrrolidone) Diblock Copolymers" J. Polym. Sci. A Polym. Chem 2014, 52, 2725-2737.

(199) Qiao, Z. Y.; Ji, R.; Huang, X. N.; Du, F. S.; Zhang, R.; Liang, D. H.; Li, Z. C. "Polymersomes from Dual Responsive Block Copolymers: Drug Encapsulation by Heating and Acid-Triggered Release" Biomacromolecules 2013, 14, 1555-1563.

(200) Hsu, S. P.; Chu, I. M.; Yang, J. D. "Thermo- and pH-Responsive Polymersomes of Poly(alpha,beta-N-substituted-DL-aspartamide)s" J. Appl. Polym. Sci. 2012, 125, 133-144.

(201) Lai, M. H.; Jeong, J. H.; DeVolder, R. J.; Brockman, C.; Schroeder, C.; Kong, H. "Ellipsoidal Polyaspartamide Polymersomes with Enhanced Cell-Targeting Ability" Adv. Funct. Mater. 2012, 22, 3239-3246.

(202) Zhuang, Z. L.; Cai, C. H.; Jiang, T.; Lin, J. P.; Yang, C. Y. "Self-assembly behavior of rod-coil-rod polypeptide block copolymers" Polymer 2014, 55, 602-610.

144 
(203) Iatrou, H.; Dimas, K.; Gkikas, M.; Tsimblouli, C.; Sofianopoulou, S. "Polymersomes from Polypeptide Containing Triblock Co- and Terpolymers for Drug Delivery against Pancreatic Cancer: Asymmetry of the External Hydrophilic Blocks" Macromol. Biosci. 2014, 14, 1222-1238.

$\mathrm{Hu}$, H.; Liu, G. J. "Miktoarm Star Copolymer Capsules Bearing pH-Responsive Nanochannels" Macromolecules 2014, 47, 5096-5103.

Gaitzsch, J.; Canton, I.; Appelhans, D.; Battaglia, G.; Voit, B. "Cellular Interactions with Photo-Cross-Linked and pH-Sensitive Polymersomes: Biocompatibility and Uptake Studies" Biomacromolecules 2012, 13, 4188-4195.

(206) Gaitzsch, J.; Appelhans, D.; Janke, A.; Strempel, M.; Schwille, P.; Voit, B. "Cross-linked and pH sensitive supported polymer bilayers from polymersomes - studies concerning thickness, rigidity and fluidity" Soft Matter 2014, 10, 66-73.

(207) Gupta, S.; Kuckling, D.; Kretschmer, K.; Choudhary, V.; Adler, H. J. "Synthesis and characterization of stimuli-sensitive micro- and nanohydrogels based on photocrosslinkable poly(dimethylaminoethyl methacrylate)" J. Polym. Sci. A Polym. Chem 2007, 45, 669-679. Hoffmann, R.; Woodward, R. B. "SELECTION RULES FOR CONCERTED CYCLOADDITION REACTIONS" J. Am. Chem. Soc. 1965, 87, 2046-\&.

(209) Du, J. Z.; Armes, S. P. "pH-responsive vesicles based on a hydrolytically self-cross-linkable copolymer" J. Am. Chem. Soc. 2005, 127, 12800-12801.

Chambon, P.; Blanazs, A.; Battaglia, G.; Armest, S. P. "How Does Cross-Linking Affect the Stability of Block Copolymer Vesicles in the Presence of Surfactant?" Langmuir 2012, 28, 1196-1205. 
(211) Zhao, Q.; Wang, Y.; Yan, Y.; Huang, J. B. "Smart Nanocarrier: Self-Assembly of Bacteria-like Vesicles with Photoswitchable Cilia" ACS Nano 2014, 8, 11341-11349.

(212) Mazzier, D.; Maran, M.; Perucchin, O. P.; Crisma, M.; Zerbetto, M.; Causin, V.; Toniolo, C.; Moretto, A. "Photoresponsive Supramolecular Architectures Based on Polypeptide Hybrids" Macromolecules 2014, 47, 7272-7283.

(213) Blasco, E.; Schmidt, B. V. K. J.; Barner-Kowollik, C.; Pinol, M.; Oriol, L. "A Novel Photoresponsive Azobenzene-Containing Miktoarm Star Polymer: Self-Assembly and Photoresponse Properties" Macromolecules 2014, 47, 3693-3700.

(214) Blasco, E.; del Barrio, J.; Sanchez-Somolinos, C.; Pinol, M.; Oriol, L. "Light induced molecular release from vesicles based on amphiphilic linear-dendritic block copolymers" Polym. Chem. 2013, 4, 2246-2254.

(215) Xia, D. Y.; Yu, G. C.; Li, J. Y.; Huang, F. H. "Photo-responsive self-assembly based on a water-soluble pillar[6] arene and an azobenzene-containing amphiphile in water" Chem. Comm. 2014, 50, 3606-3608.

(216) Cabane, E.; Malinova, V.; Meier, W. "Synthesis of Photocleavable Amphiphilic Block Copolymers: Toward the Design of Photosensitive Nanocarriers" Macromol. Chem. Phys. 2010, 211, 1847-1856.

(217) Liu, G.; Zhou, L. Z.; Guan, Y. F.; Su, Y.; Dong, C. M. "Multi-Responsive Polypeptidosome: Characterization, Morphology Transformation, and Triggered Drug Delivery" Macromol. Rapid Commun. 2014, 35, 1673-1678.

(218) Cerritelli, S.; Velluto, D.; Hubbell, J. A. "PEG-SS-PPS: Reduction-Sensitive Disulfide Block Copolymer Vesicles for Intracellular Drug Delivery" Biomacromolecules 2007, 8, 1966-1972. 
(219) Jia, L.; Cui, D.; Bignon, J.; Di Cicco, A.; Wdzieczak-Bakala, J.; Liu, J. M.; Li, M. H. "Reduction-Responsive Cholesterol-Based Block Copolymer Vesicles for Drug Delivery" Biomacromolecules 2014, 15, 2206-2217.

(220) Napoli, A.; Valentini, M.; Tirelli, N.; Mueller, M.; Hubbell, J. A. "Oxidation-responsive polymeric vesicles" Nat Mater 2004, 3, 183-189.

(221) Atzet, S.; Curtin, S.; Trinh, P.; Bryant, S.; Ratner, B. "Degradable Poly(2-hydroxyethyl methacrylate)-co-polycaprolactone Hydrogels for Tissue Engineering Scaffolds" Biomacromolecules 2008, 9, 3370-3377.

(222) Lee, J. S.; Feijen, J. "Biodegradable polymersomes as carriers and release systems for paclitaxel using Oregon Green (R) 488 labeled paclitaxel as a model compound" J Control Release 2012, 158, 312-318.

(223) Tamate, R.; Ueki, T.; Yoshida, R. "Self-Beating Artifi cial Cells: Design of Cross-Linked Polymersomes Showing Self-Oscillating Motion" Adv. Mater. 2015, 27, 837-842.

(224) Mabire, A. B.; Robin, M. P.; Willcock, H.; Pitto-Barry, A.; Kirby, N.; O'Reilly, R. K. "Dual effect of thiol addition on fluorescent polymeric micelles: ON-to-OFF emissive switch and morphology transition" Chem. Comm. 2014, 50, 11492-11495.

(225) Azzam, T.; Eisenberg, A. "Fully Collapsed (Kippah) Vesicles: Preparation and Characterization" Langmuir 2010, 26, 10513-10523.

(226) Kao, K.-C.; Tsou, C.-J.; Mou, C.-Y. "Collapsed (kippah) hollow silica nanoparticles" Chem. Comm. 2012, 48, 3454-3456.

(227) Chen, T.; Du, B.; Fan, Z. "Facile Fabrication of Polymer Nanocapsules with Cross-Linked Organic-Inorganic Hybrid Walls" Langmuir 2012, 28, 11225-11231.

147 
(228) Kim, K. T.; Zhu, J. H.; Meeuwissen, S. A.; Cornelissen, J. J. L. M.; Pochan, D. J.; Nolte, R. J. M.; van Hest, J. C. M. "Polymersome Stomatocytes: Controlled Shape Transformation in Polymer Vesicles" J. Am. Chem. Soc. 2010, 132, 12522-12524.

(229)

Rikken, R. S. M.; Kerkenaar, H. H. M.; Nolte, R. J. M.; Maan, J. C.; van Hest, J. C. M.; Christianen, P. C. M.; Wilson, D. A. "Probing morphological changes in polymersomes with magnetic birefringence" Chem. Comm. 2014, 50, 5394-5396.

$(230)$

Rikken, R. S. M.; Nolte, R. J. M.; Maan, J. C.; van Hest, J. C. M.; Wilson, D. A.; Christianen, P. C. M. "Manipulation of micro- and nanostructure motion with magnetic fields" Soft Matter 2014, 10, 1295-1308.

(231) Wilson, D. A.; Nolte, R. J. M.; van Hest, J. C. M. "Autonomous movement of platinum-loaded stomatocytes" Nature Chemistry 2012, 4, 268-274.

(232) Wilson, D. A.; Nolte, R. J. M.; van Hest, J. C. M. "Entrapment of Metal Nanoparticles in Polymer Stomatocytes" J Am Chem Soc 2012, 134, 9894-9897.

(233) Wilson, D. A.; de Nijs, B.; van Blaaderen, A.; Nolte, R. J. M.; van Hest, J. C. M. "Fuel concentration dependent movement of supramolecular catalytic nanomotors" Nanoscale 2013, $5,1315-1318$.

(234) Meeuwissen, S. A.; Bruekers, S. M. C.; Chen, Y. C.; Pochan, D. J.; van Hest, J. C. M. "Spontaneous shape changes in polymersomes via polymer/polymer segregation" Polym. Chem. 2014, 5, 489-501.

LoPresti, C.; Massignani, M.; Fernyhough, C.; Blanazs, A.; Ryan, A. J.; Madsen, J.; Warren, N. J.; Armes, S. P.; Lewis, A. L.; Chirasatitsin, S.; Engler, A. J.; Battaglia, G. "Controlling Polymersome Surface Topology at the Nanoscale by Membrane Confined Polymer/Polymer 
Phase Separation" ACS Nano 2011, 5, 1775-1784.

(236) Massignani, M.; LoPresti, C.; Blanazs, A.; Madsen, J.; Armes, S. P.; Lewis, A. L.; Battaglia, G. "Controlling Cellular Uptake by Surface Chemistry, Size, and Surface Topology at the Nanoscale" Small 2009, 5, 2424-2432.

Ruiz-Perez, L.; Madsen, J.; Themistou, E.; Gaitzsch, J.; Armes, S. P.; Battaglia, G. "Nanoscale detection of metal-labeled copolymers in patchy polymersomes" Poly. Chem. 2015, 6, 2065-2068.

Liu, Q. M.; Chen, S.; Chen, J.; Du, J. Z. "An Asymmetrical Polymer Vesicle Strategy for Significantly Improving T-1 MRI Sensitivity and Cancer-Targeted Drug Delivery" Macromolecules 2015, 48, 739-749.

(239) Xu, W.; Ledin, P. A.; Plamper, F. A.; Synatschke, C. V.; Muller, A. H. E.; Tsukruk, V. V. "Multiresponsive Microcapsules Based on Multilayer Assembly of Star Polyelectrolytes" Macromolecules 2014, 47, 7858-7868.

(240) Städler, B.; Price, A. D.; Chandrawati, R.; Hosta-Rigau, L.; Zelikin, A. N.; Caruso, F. "Polymer hydrogel capsules: en route toward synthetic cellular systems" Nanoscale 2009, 1, 68-73.

(241) Chandrawati, R.; Chong, S. F.; Zelikin, A. N.; Hosta-Rigau, L.; Städler, B.; Caruso, F. "Degradation of liposomal subcompartments in PEGylated capsosomes" Soft Matter 2011, 7, 9638-9646.

(242) Hosta-Rigau, L.; Chung, S. F.; Postma, A.; Chandrawati, R.; Städler, B.; Caruso, F. "Capsosomes with “Free - Floating” Liposomal Subcompartments" Adv. Mater. 2011, 23, 4082-4087. 
(243) Chandrawati, R.; van Koeverden, M. P.; Lomas, H.; Caruso, F. "Multicompartment Particle Assemblies for Bioinspired Encapsulated Reactions" J. Phys. Chem. Lett. 2011, 2, 2639-2649.

(244) Chandrawati, R.; Odermatt, P. D.; Chong, S. F.; Price, A. D.; Städler, B.; Caruso, F. "Triggered Cargo Release by Encapsulated Enzymatic Catalysis in Capsosomes" Nano Lett. 2011, 11, 4958-4963.

(245) Ringsdorf, H. 3rd EURESCO Conference on Supramolecular Chemistry: Molecular Recognition and Drug-Receptor Interactions, Salamanca (Spain), Aug 29 - Sept 3, 1996.

(246) Li, Z.; Kesselman, E.; Talmon, Y.; Hillmyer, M. A.; Lodge, T. P. "Multicompartment micelles from ABC miktoarm stars in water" Science 2004, 306, 98.

(247) Gröschel, A. H.; Walther, A.; Löbling, T. I.; Schmelz, J.; Hanisch, A.; Schmalz, H.; Müller, A. H. E. "Facile, Solution-Based Synthesis of Soft, Nanoscale Janus Particles with Tunable Janus Balance" J. Am. Chem. Soc. 2012, 134, 13850-13860.

(248) Lutz, J. F.; Laschewsky, A. "Multicompartment Micelles: Has the Long - Standing Dream Become a Reality?" Macromol. Chem. Phys. 2005, 206, 813-817.

Paleos, C. M.; Tsiourvas, D.; Sideratou, Z.; Pantos, A. "Formation of artificial multicompartment vesosome and dendrosome as prospected drug and gene delivery carriers" $J$ Control Release 2013, 170, 141-152.

(250) Marguet, M.; Sandre, O.; Lecommandoux, S. "Polymersomes in "Gelly" Polymersomes: Toward Structural Cell Mimicry" Langmuir 2012, 28, 2035-2043.

(251) Zhang, S.; Moussodia, R.-O.; Vertesy, S.; Andre, S.; Klein, M. L.; Gabius, H.-J.; Percec, V. "Unraveling functional significance of natural variations in structural aspects of a human galectin by glycodendrimersomes with programmable glycan surface" Proc. Natl. Acad. Sci. 
U.S.A 2015, 112, 5585-5590.

Zhang, S.; Moussodia, R.-O.; Murzeau, C.; Sun, H.-J.; Klein, M. L.; Vertesy, S.; Andre, S.;

Roy, R.; Gabius, H.-J.; Percec, V. "Dissecting Molecular Aspects of Cell Interactions Using Glycodendrimersomes with Programmable Glycan Presentation and Engineered Human Lectins" Angew. Chem. Int. Ed. 2015, 54, 4036-4040.

Zhang, S.; Sun, H.-J.; Hughes, A. D.; Moussodia, R.-O.; Bertin, A.; Chen, Y.; Pochan, D. J.; Heiney, P. A.; Klein, M. L.; Percec, V. "Self-assembly of amphiphilic Janus dendrimers into uniform onion-like dendrimersomes with predictable size and number of bilayers" Proc. Natl. Acad. Sci. U.S.A 2014, 111, 9058-9063.

Zhang, S.; Sun, H.-J.; Hughes, A. D.; Draghici, B.; Lejnieks, J.; Leowanawat, P.; Bertin, A.; De Leon, L. O.; Kulikov, O. V.; Chen, Y.; Pochan, D. J.; Heiney, P. A.; Percec, V. "'Single-Single" Amphiphilic Janus Dendrimers Self-Assemble into Uniform Dendrimersomes with Predictable Size" ACS Nano 2014, 8, 1554-1565.

Zhang, S.; Moussodia, R.-O.; Sun, H.-J.; Leowanawat, P.; Muncan, A.; Nusbaum, C. D.; Chelling, K. M.; Heiney, P. A.; Klein, M. L.; Andre, S.; Roy, R.; Gabius, H.-J.; Percec, V. "Mimicking Biological Membranes with Programmable Glycan Ligands Self-Assembled from Amphiphilic Janus Glycodendrimers" Angew. Chem. Int. Ed. 2014, 53, 10899-10903.

Percec, V.; Leowanawat, P.; Sun, H.-J.; Kulikov, O.; Nusbaum, C. D.; Tran, T. M.; Bertin, A.; Wilson, D. A.; Peterca, M.; Zhang, S.; Kamat, N. P.; Vargo, K.; Moock, D.; Johnston, E. D.; Hammer, D. A.; Pochan, D. J.; Chen, Y.; Chabre, Y. M.; Shiao, T. C.; Bergeron-Brlek, M.; Andre, S.; Roy, R.; Gabius, H.-J.; Heiney, P. A. "Modular Synthesis of Amphiphilic Janus Glycodendrimers and Their Self-Assembly into Glycodendrimersomes and Other Complex

151 
Architectures with Bioactivity to Biomedically Relevant Lectins" J. Am. Chem. Soc. 2013, $135,9055-9077$.

(257) Peterca, M.; Percec, V.; Leowanawat, P.; Bertin, A. "Predicting the Size and Properties of Dendrimersomes from the Lamellar Structure of Their Amphiphilic Janus Dendrimers" J. Am. Chem. Soc. 2011, 133, 20507-20520.

(258) Rosen, B. M.; Wilson, C. J.; Wilson, D. A.; Peterca, M.; Imam, M. R.; Percec, V. "Dendron-mediated self-assembly, disassembly, and self-organization of complex systems" Chem. Rev. 2009, 109, 6275-6540.

(259) Städler, B.; Chandrawati, R.; Price, A. D.; Chong, S. F.; Breheney, K.; Postma, A.; Connal, L. A.; Zelikin, A. N.; Caruso, F. "A Microreactor with Thousands of Subcompartments: Enzyme - Loaded Liposomes within Polymer Capsules" Angew. Chem. Int. Ed. 2009, 48, 4859-4862.

(260) Städler, B.; Chandrawati, R.; Goldie, K.; Caruso, F. "Capsosomes: subcompartmentalizing polyelectrolyte capsules using liposomes" Langmuir 2009, 25, 6725-6732.

(261) Fu, Z.; Ochsner, M. A.; De Hoog, H. P. M.; Tomczak, N.; Nallani, M. "Multicompartmentalized polymersomes for selective encapsulation of biomacromolecules" Chem. Comm. 2011, 47, 2862-2864.

(262) Li, G.; Lei, C.; Wang, C.; Neoh, K.; Kang, E.; Yang, X. "Narrowly dispersed double-walled concentric hollow polymeric microspheres with independent $\mathrm{pH}$ and temperature sensitivity" Macromolecules 2008, 41, 9487-9490.

Chiu, H.-C.; Lin, Y.-W.; Huang, Y.-F.; Chuang, C.-K.; Chern, C.-S. "Polymer Vesicles Containing Small Vesicles within Interior Aqueous Compartments and $\mathrm{pH}$-Responsive Transmembrane Channels" Angew. Chem. Int. Ed. 2008, 47, 1875-1878.

152 
(264) Thompson, K. L.; Chambon, P.; Verber, R.; Armes, S. P. "Can Polymersomes Form Colloidosomes?" J. Am. Chem. Soc. 2012, 134, 12450-12453.

(265) Shum, H. C.; Zhao, Y.; Kim, S. H.; Weitz, D. A. "Multicompartment polymersomes from double emulsions" Angew. Chem. Int. Ed. 2011, 50, 1648-1651.

Nisisako, T.; Okushima, S.; Torii, T. "Controlled formulation of monodisperse double emulsions in a multiple-phase microfluidic system" Soft Matter 2005, 1, 23-27.

Sanson, C.; Schatz, C.; Le Meins, J.-F. o.; Brûlet, A.; Soum, A.; Lecommandoux, S. b. "Biocompatible and Biodegradable Poly(trimethylene carbonate)-b-Poly(1-glutamic acid) Polymersomes: Size Control and Stability" Langmuir 2009, 26, 2751-2760. Mabrouk, E.; Cuvelier, D.; Pontani, L.-L.; Xu, B.; Levy, D.; Keller, P.; Brochard-Wyart, F.; Nassoy, P.; Li, M.-H. "Formation and material properties of giant liquid crystal polymersomes" Soft Matter 2009, 5, 1870-1878.

Siti, W.; de Hoog, H.-P. M.; Fischer, O.; Shan, W. Y.; Tomczak, N.; Nallani, M.; Liedberg, B. "An intercompartmental enzymatic cascade reaction in channel-equipped polymersome-in-polymersome architectures" J. Mater. Chem. B 2014, 2, 2733-2737.

(270) Hosta-Rigau, L.; Städler, B.; Yan, Y.; Nice, E. C.; Heath, J. K.; Albericio, F.; Caruso, F. "Capsosomes with multilayered subcompartments: assembly and loading with hydrophobic cargo" Adv. Funct. Mater. 2010, 20, 59-66.

(271) Hosta-Rigau, L.; Chandrawati, R.; Saveriades, E.; Odermatt, P. D.; Postma, A.; Ercole, F.; Breheney, K.; Wark, K. L.; Städler, B.; Caruso, F. "Noncovalent Liposome Linkage and Miniaturization of Capsosomes for Drug Delivery" Biomacromolecules 2010, 11 3548-3555.

(272) Chandrawati, R.; Städler, B.; Postma, A.; Connal, L. A.; Chong, S. F.; Zelikin, A. N.; Caruso, 
F. "Cholesterol-mediated anchoring of enzyme-loaded liposomes within disulfide-stabilized polymer carrier capsules" Biomaterials 2009, 30, 5988-5998.

(273) Zelikin, A. N.; Quinn, J. F.; Caruso, F. "Disulfide cross-linked polymer capsules: en route to biodeconstructible systems" Biomacromolecules 2006, 7, 27-30.

(274) Lomas, H.; Johnston, A. P. R.; Such, G. K.; Zhu, Z.; Liang, K.; van Koeverden, M. P.; Alongkornchotikul, S.; Caruso, F. "Polymersome-Loaded Capsules for Controlled Release of DNA" Small 2011, 7, 2109-2119.

(275) Lee, M. H.; Hribar, K. C.; Brugarolas, T.; Kamat, N. P.; Burdick, J. A.; Lee, D. "Harnessing Interfacial Phenomena to Program the Release Properties of Hollow Microcapsules" Adv. Funct. Mater. 2012, 22, 131-138.

(276) Li, G. L.; Tai, C. A.; Neoh, K.; Kang, E.; Yang, X. "Hybrid nanorattles of metal core and stimuli-responsive polymer shell for confined catalytic reactions" Polym. Chem. 2011, 2, 1368-1374.

(277) Kinning, D. J.; Winey, K. I.; Thomas, E. L. "Structural transitions from spherical to nonspherical micelles in blends of poly(styrene-butadiene) diblock copolymer and polystyrene homopolymers" Macromolecules 1988, 21, 3502-3506.

(278) Zipfel, J.; Lindner, P.; Tsianou, M.; Alexandridis, P.; Richtering, W. "Shear-Induced Formation of Multilamellar Vesicles (“Onions”) in Block Copolymers" Langmuir 1999, 15, 2599-2602.

(279) Shen, H.; Eisenberg, A. "Control of Architecture in Block-Copolymer Vesicles" Angew. Chem. Int. Ed. 2000, 39, 3310-3312.

(280) Li, L.; Rosenthal, M.; Zhang, H.; Hernandez, J. J.; Drechsler, M.; Phan, K. H.; Rütten, S.; Zhu, X.; Ivanov, D. A.; Möller, M. "Light-Switchable Vesicles from Liquid-Crystalline 
Homopolymer-Surfactant Complexes" Angew. Chem. Int. Ed. 2012, 51, 11616-11619.

(281) Mai, Y.; Eisenberg, A. "Self-assembly of block copolymers" Chem. Soc. Rev. 2012, 41, 5969-5985.

(282) Li, M.; Huang, X.; Tang, T. Y. D.; Mann, S. "Synthetic cellularity based on non-lipid micro-compartments and protocell models" Curr. Opin. Chem. Biol. 2014, 22, 1-11.

Palivan, C. G.; Fischer-Onaca, O.; Delcea, M.; Itel, F.; Meier, W. "Protein-polymer nanoreactors for medical applications" Chem. Soc. Rev. 2012, 41, 2800-2823.

(284) Liang, K.; Such, G. K.; Johnston, A. P. R.; Zhu, Z.; Ejima, H.; Richardson, J. J.; Cui, J.; Caruso, F. "Endocytic pH-Triggered Degradation of Nanoengineered Multilayer Capsules" Adv. Mater. 2014, 26, 1901-1905.

(285) Liu, G.; Wang, X.; Hu, J.; Zhang, G.; Liu, S. "Self-Immolative Polymersomes for High-Efficiency Triggered Release and Programmed Enzymatic Reactions" J. Am. Chem. Soc. 2014, 136, 7492-7497.

(286) Viger, M. L.; Sheng, W.; Dore, K.; Alhasan, A. H.; Carling, C.-J.; Lux, J.; Lux, C. d. G.; Grossman, M.; Malinow, R.; Almutairi, A. "Near-Infrared-Induced Heating of Confined Water in Polymeric Particles for Efficient Payload Release" ACS Nano 2014, 8, 4815-4826.

(287) Yi, Q.; Sukhorukov, G. B. "UV light stimulated encapsulation and release by polyelectrolyte microcapsules" Adv. Colloid Interface Sci. 2014, 207, 280-289.

Zhang, Y.; Teo, $\quad$ B. $\quad$ M.; Goldie, K. N.; Stadler, B. "Poly(N-isopropylacrylamide)/Poly(dopamine) Capsules" Langmuir 2014, 30, 5592-5598.

(289) van Dongen, S. F. M.; Verdurmen, W. P. R.; Peters, R. J. R. W.; Nolte, R. J. M.; Brock, R.; van Hest, J. C. M. "Cellular Integration of an Enzyme-Loaded Polymersome Nanoreactor" Angew. 
Chem. Int. Ed. 2010, 49, 7213-7216.

(290) Kuiper, S. M.; Nallani, M.; Vriezema, D. M.; Cornelissen, J. J. L. M.; van Hest, J. C. M.; Nolte, R. J. M.; Rowan, A. E. "Enzymes containing porous polymersomes as nano reaction vessels for cascade reactions" Org. Biomol. Chem. 2008, 6, 4315-4318.

(291) Langowska, K.; Palivan, C. G.; Meier, W. "Polymer nanoreactors shown to produce and release antibiotics locally" Chem. Comm. 2013, 49, 128-130.

(292) Onaca, O.; Hughes, D. W.; Balasubramanian, V.; Grzelakowski, M.; Meier, W.; Palivan, C. G. "SOD Antioxidant Nanoreactors: Influence of Block Copolymer Composition on the Nanoreactor Efficiency" Macromol. Biosci. 2010, 10, 531-538.

(293) Axthelm, F.; Casse, O.; Koppenol, W. H.; Nauser, T.; Meier, W.; Palivan, C. G. "Antioxidant nanoreactor based on superoxide dismutase encapsulated in superoxide-permeable vesicles" $J$. Phys. Chem. B 2008, 112, 8211-8217.

(294) Wang, Z.; van Oers, M. C. M.; Rutjes, F. P. J. T.; van Hest, J. C. M. "Polymersome Colloidosomes for Enzyme Catalysis in a Biphasic System" Angew. Chem. Int. Ed. 2012, 51, 10746-10750.

(295) Meeuwissen, S. A.; Rioz-Martinez, A.; de Gonzalo, G.; Fraaije, M. W.; Gotor, V.; van Hest, J. C. M. "Cofactor regeneration in polymersome nanoreactors: enzymatically catalysed Baeyer-Villiger reactions" J Mater Chem 2011, 21, 18923-18926.

(296) Spulber, M.; Baumann, P.; Saxer, S. S.; Pieles, U.; Meier, W.; Bruns, N. "Poly (N-vinylpyrrolidone)-poly (dimethylsiloxane)-based polymersome nanoreactors for laccase-catalyzed biotransformations" Biomacromolecules 2014, 15, 1469-1475.

(297) Yildiz, U. H.; De Hoog, H.-P. M.; Fu, Z.; Tomczak, N.; Parikh, A. N.; Nallani, M.; Liedberg, 
B. "Third-Party ATP Sensing in Polymersomes: A Label-Free Assay of Enzyme Reactions in Vesicular Compartments" Small 2014, 10, 442-447.

(298)

Vriezema, D. M.; Comellas Aragonès, M.; Elemans, J. A. A. W.; Cornelissen, J. J. L. M.;

Rowan, A. E.; Nolte, R. J. M. "Self-Assembled Nanoreactors" Chem. Rev. 2005, 105, 1445-1490.

(299) Rosenkranz, T.; Katranidis , A.; Atta, D.; Gregor, I.; Enderlein, J.; Grzelakowski, M.; Rigler, P.; Meier, W.; Fitter, J. "Observing Proteins as Single Molecules Encapsulated in Surface-Tethered Polymeric Nanocontainers" ChemBioChem 2009, 10, 702-709.

(300) Comellas-Aragones, M.; Engelkamp, H.; Claessen, V. I.; Sommerdijk, N. A. J. M.; Rowan, A. E.; Christianen, P. C. M.; Maan, J. C.; Verduin, B. J. M.; Cornelissen, J. J. L. M.; Nolte, R. J. M. "A virus-based single-enzyme nanoreactor" Nat. Nano. 2007, 2, 635-639.

(301) van Dongen, S. F. M.; Nallani, M.; Cornelissen, J. L. L. M.; Nolte, R. J. M.; van Hest, J. C. M. "A Three-Enzyme Cascade Reaction through Positional Assembly of Enzymes in a Polymersome Nanoreactor" Chem. Eur. J. 2009, 15, 1107-1114.

(302) Vriezema, D. M.; Garcia, P. M. L.; Sancho Oltra, N.; Hatzakis, N. S.; Kuiper, S. M.; Nolte, R. J. M.; Rowan, A. E.; van Hest, J. C. M. "Positional Assembly of Enzymes in Polymersome Nanoreactors for Cascade Reactions" Angew. Chem. Int. Ed. 2007, 46, 7378-7382.

(303) Baumann, P.; Balasubramanian, V.; Onaca-Fischer, O.; Sienkiewicz, A.; Palivan, C. G. "Light-responsive polymer nanoreactors: a source of reactive oxygen species on demand" Nanoscale 2013, 5, 217-224.

(304) Brinkhuis, R. P.; Rutjes, F. P. J. T.; van Hest, J. C. M. "Polymeric vesicles in biomedical applications" Polym. Chem. 2011, 2, 1449-1462. 
(305) Valade, D.; Jeon, Y.; Kessel, S.; Monteiro, M. J. "Influence of the Z - group on the RAFT mediated polymerizations in nanoreactors" J. Polym. Sci. A Polym. Chem 2012, 50, 4762-4771.

(306) Zetterlund, P. B. "Controlled/living radical polymerization in nanoreactors: compartmentalization effects" Polym. Chem. 2011, 2, 534-549.

(307) Zhang, M.; Zhang, W. "Reversible addition - fragmentation chain transfer polymerization of a typical hydrophobic monomer of styrene within microreactor of shell - corona hollow microspheres suspending in water" J. Polym. Sci. A Polym. Chem 2010, 48, 5446-5455.

(308) Asua, J. M. "Miniemulsion polymerization" Prog. Polym. Sci. 2002, 27, 1283-1346.

(309) Landfester, K. "Polyreactions in Miniemulsions" Macromol. Rapid Commun. 2001, 22, 896-936.

(310) Chow, P. Y.; Gan, L. M. "Microemulsion Polymerizations and Reactions" Adv. Polym. Sci. 2008, 175, 257-298.

(311) Zetterlund, P. B.; Kagawa, Y.; Okubo, M. "Controlled/Living Radical Polymerization in Dispersed Systems" Chem. Rev. 2008, 108, 3747-3794.

(312) Kent, P.; Saunders, B. R. "The Role of Added Electrolyte in the Stabilization of Inverse Emulsions" J. Colloid Interface Sci. 2001, 242, 437-442.

(313) Landfester, K.; Willert, M.; Antonietti, M. "Preparation of Polymer Particles in Nonaqueous Direct and Inverse Miniemulsions" Macromolecules 2000, 33, 2370-2376.

(314) Sukhorukov, G. B.; Susha, A. S.; Davis, S.; Leporatti, S.; Donath, E.; Hartmann, J.; Möhwald, H. "Precipitation of Inorganic Salts inside Hollow Micrometer-Sized Polyelectrolyte Shells" J. Colloid Interface Sci. 2002, 247, 251-254. 
(315) Li, W.; Sha, X.; Dong, W.; Wang, Z. "Synthesis of stable hollow silica microspheres with mesoporous shell in nonionic W/O emulsion" Chem. Comm. 2002, 2434-2435.

(316) Jesionowski, T. "Influence of aminosilane surface modification and dyes adsorption on zeta potential of spherical silica particles formed in emulsion system" Colloid Surf. A-Physicochem. Eng. Asp. 2003, 222, 87-94.

(317) Andersson, M.; Österlund, L.; Ljungström, S.; Palmqvist, A. "Preparation of Nanosize Anatase and Rutile TiO2 by Hydrothermal Treatment of Microemulsions and Their Activity for Photocatalytic Wet Oxidation of Phenol" J. Phys. Chem. B 2002, 106, 10674-10679. Li, M.; Mann, S. "Emergent Nanostructures: Water-Induced Mesoscale Transformation of Surfactant-Stabilized Amorphous Calcium Carbonate Nanoparticles in Reverse Microemulsions" Adv. Funct. Mater. 2002, 12, 773-779.

(319) Horecha, M.; Kaul, E.; Horechyy, A.; Stamm, M. "Polymer microcapsules loaded with Ag nanocatalyst as active microreactors" $J$. Mater. Chem. A 2014, 2, 7431-7438.

(320) Sukhorukov, G. B.; Brumen, M.; Donath, E.; Möhwald, H. "Hollow Polyelectrolyte Shells: Exclusion of Polymers and Donnan Equilibrium" J. Phys. Chem. B 1999, 103, 6434-6440.

(321) Sukhorukov, G.; Dähne, L.; Hartmann, J.; Donath, E.; Möhwald, H. "Controlled Precipitation of Dyes into Hollow Polyelectrolyte Capsules Based on Colloids and Biocolloids" Adv. Mater. 2000, $12,112-115$.

(322) Ghan, R.; Shutava, T.; Patel, A.; John, V. T.; Lvov, Y. "Enzyme-Catalyzed Polymerization of Phenols within Polyelectrolyte Microcapsules" Macromolecules 2004, 37, 4519-4524.

(323) Shchukin, D. G.; Sukhorukov, G. B. "Nanoparticle synthesis in engineered organic nanoscale reactors" Adv. Mater. 2004, 16, 671-682. 
(324) Shchukin, D. G.; Dong, W.; Sukhorukov, G. B. "Spatially Confined Tungstate Ion Polymerization in Microcapsules" Macromol. Rapid Commun. 2003, 24, 462-466.

(325) Shchukin, D. G.; Schattka, J. H.; Antonietti, M.; Caruso, R. A. "Photocatalytic Properties of Porous Metal Oxide Networks Formed by Nanoparticle Infiltration in a Polymer Gel Template" J. Phys. Chem. B 2003, 107, 952-957.

(326) Radtchenko, I. L.; Giersig, M.; Sukhorukov, G. B. "Inorganic Particle Synthesis in Confined Micron-Sized Polyelectrolyte Capsules" Langmuir 2002, 18, 8204-8208.

(327) Elani, Y.; Law, R. V.; Ces, O. "Vesicle-based artificial cells as chemical microreactors with spatially segregated reaction pathways" Nat. Commun. 2014, 5, 5305.

(328) Mansy, S. S.; Schrum, J. P.; Krishnamurthy, M.; Tobe, S.; Treco, D. A.; Szostak, J. W. "Template-directed synthesis of a genetic polymer in a model protocell" Nature 2008, 454, 122-U110.

(329) Nardin, C.; Thoeni, S.; Widmer, J.; Winterhalter, M.; Meier, W. "Nanoreactors based on (polymerized) ABA-triblock copolymer vesicles" Chem. Comm. 2000, 1433-1434.

(330) Baumann, P.; Spulber, M.; Dinu, I. A.; Palivan, C. G. "Cellular trojan horse based polymer nanoreactors with light-sensitive activity" J. Phys. Chem. B 2014, 118, 9361-9370.

160 


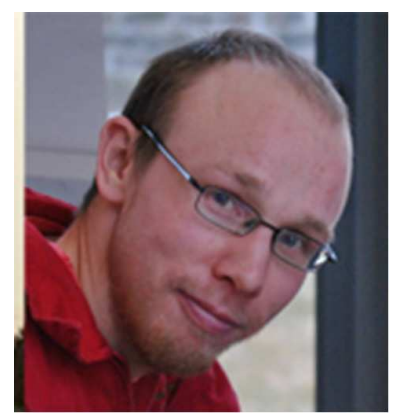

Jens Gaitzsch received his PhD from the Technische Universität Dresden (TUD) in 2013 under the guidance of Prof Brigitte Voit, conducting the research at the Leibniz Institute of Polymer Research (IPF) Dresden. The thesis was funded by a studentship of the Rosa-Luxemburg-Foundation. He then worked as a PDRA in the Group of Prof Giuseppe Battaglia at University College London before moving into a Fellowship of the German Research Association (DFG) in 2014 but remaining in the same group. In September 2015 he started working at the University of Basel in the Group of Prof. Meier. His research is focussed on the development on functional biomimetic and amphiphilic block-copolymers for biomedical applications.

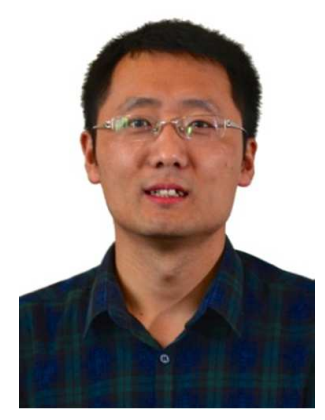

Xin Huang received his PhD from Jilin University, China, in 2009. Then he did 
one year postdoctoral research at Centre for Advanced Macromolecular Design, UNSW, Australia. In 2010, he carried out his postdoctoral research work supported by Alexander von Humboldt foundation under the guidance of Prof. Brigitte Voit at Leibniz Institute of Polymer Research Dresden, Germany. After that, in 2012 he was granted Marie Curie Research Fellowship and did two years' research in Prof. Stephen Mann's group in University of Bristol, UK. Since July 2014, he was appointed as professor at Harbin Institute of Technology, China. His main research interests lie in the construction of polymer/protein-based compartment towards protocells, artificial organelles or nanoreactors etc.

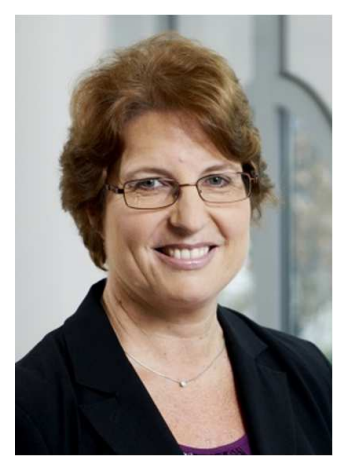

Brigitte Voit received her PhD in Macromolecular Chemistry in 1990 from University Bayreuth, Germany. After a postdoctoral work at Eastman Kodak in Rochester, USA, she went in 1992 to Technische Universität München receiving her habilitation degree in Macromolecular Chemistry there in 1996. 
In 1997 Brigitte Voit was appointed head of the Institute of Macromolecular Chemistry at the Leibniz Institute of Polymer Research (IPF) Dresden, as well as Professor for "Organic Chemistry of Polymers" at Technische Universität Dresden (TUD). Since 2002 she is in addition heading the IPF Dresden as Managing Director and Chief Scientific Officer. Her major research interest is in the synthesis of new functional polymer architectures covering topics like dendritic polymers, functional block and graft copolymers, responsive hydrogels and capsules, as well as thermo- and photo labile polymers. 


\section{TOC graphic}

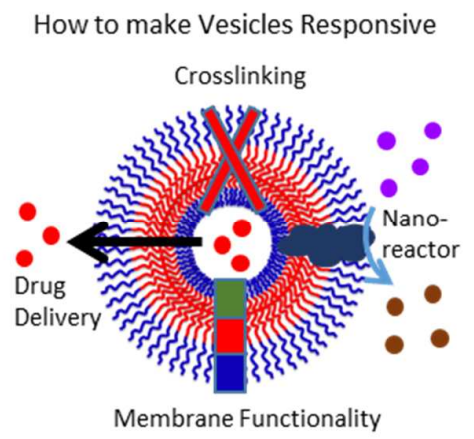

20

21

22

23

24

25

26

27

28

29

30

31

32

33

34

35

36

37

38

39

40

41

42

43

44

45

46

47

48

49

50

51

52

53

54

55

56

57

58

59

60

164 


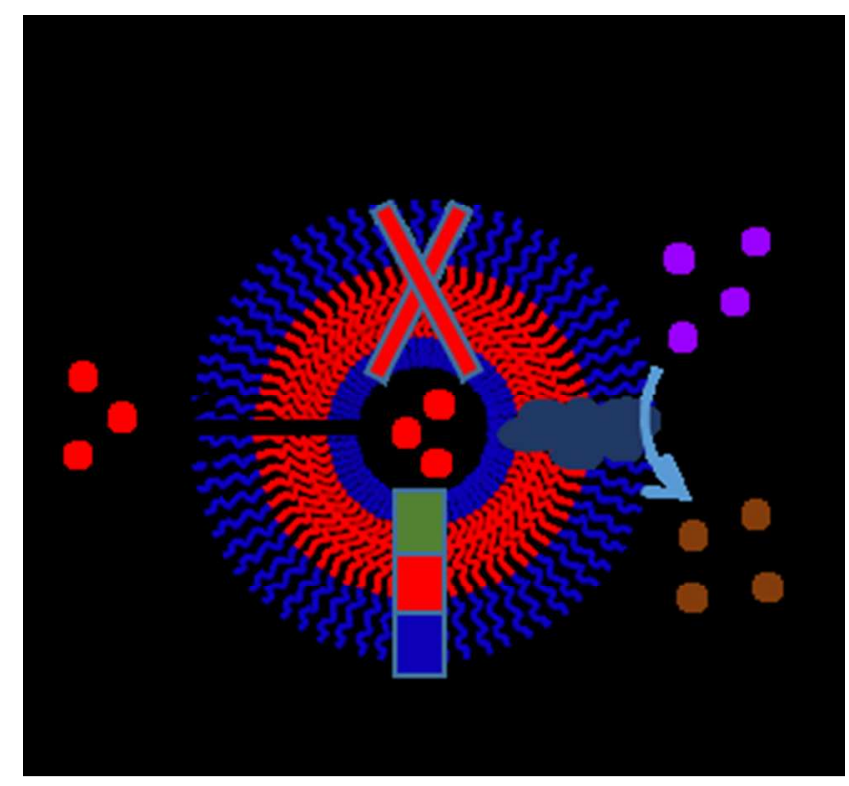

$53 \times 49 \mathrm{~mm}(150 \times 150 \mathrm{DPI})$ 


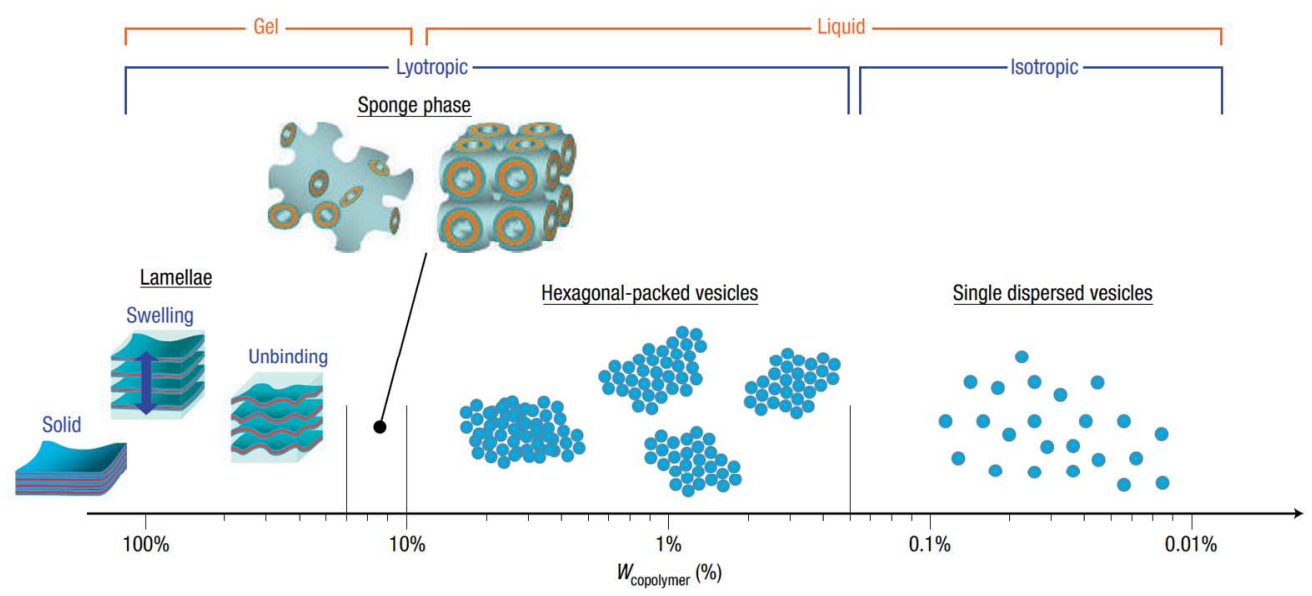

447x200mm (96 x 96 DPI) 

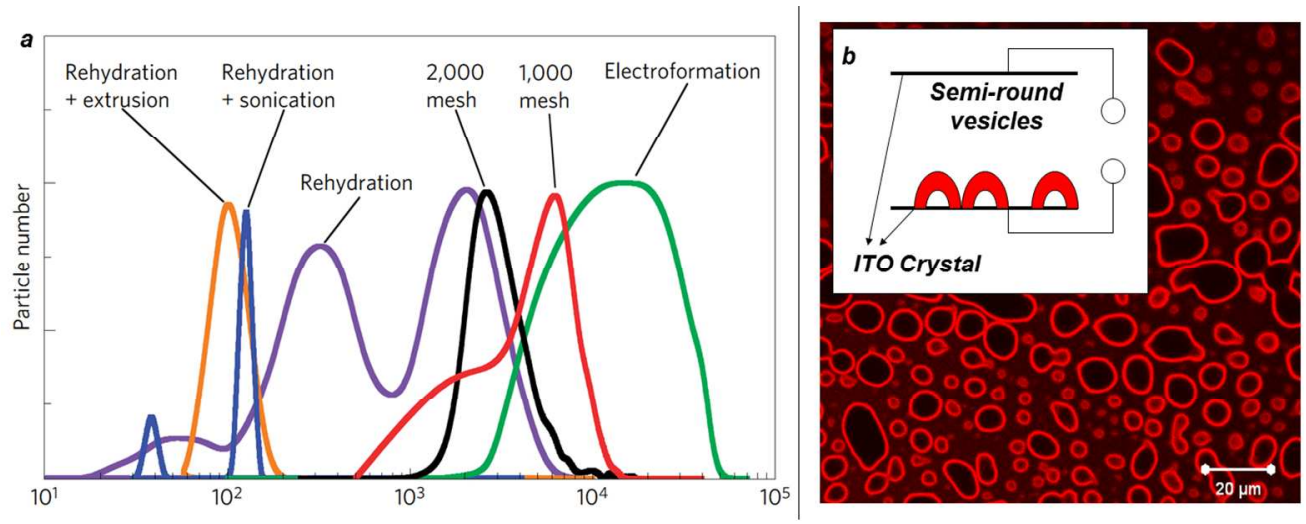

$384 \times 155 \mathrm{~mm}(96 \times 96$ DPI)

1

2

3

4

5

6
7

8

9

10

11

12

13

14

15
16
17

17

18

19

20

21

22

23

24

25

26

27

28

29

30

31

32

33

34

35

36

37

38

39

40

41

42

43

44

45

46

47

48

49

50

51

52

53

54

55

56

57

58

59

60 


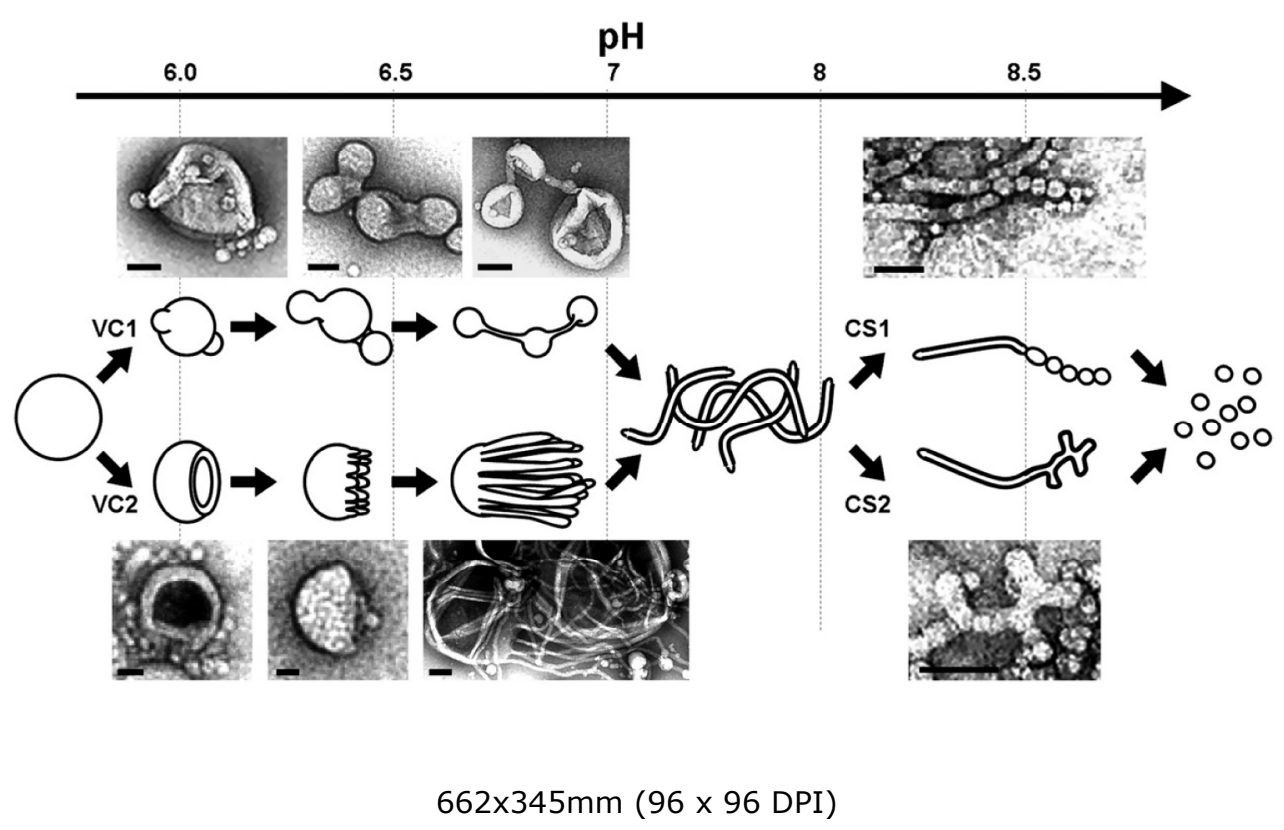




1
2
3
4
5
6
7
8
9
10
11
12
13
14
15
16
17
18
19
20
21
22
23
24
25
26
27
28
29
30
31
32
33
34
35
36
37
38
39
40
41
42
43
44
45
46
47
48
49
50
51
52
53
54
55
56
57
58
60

\section{Below CMC}

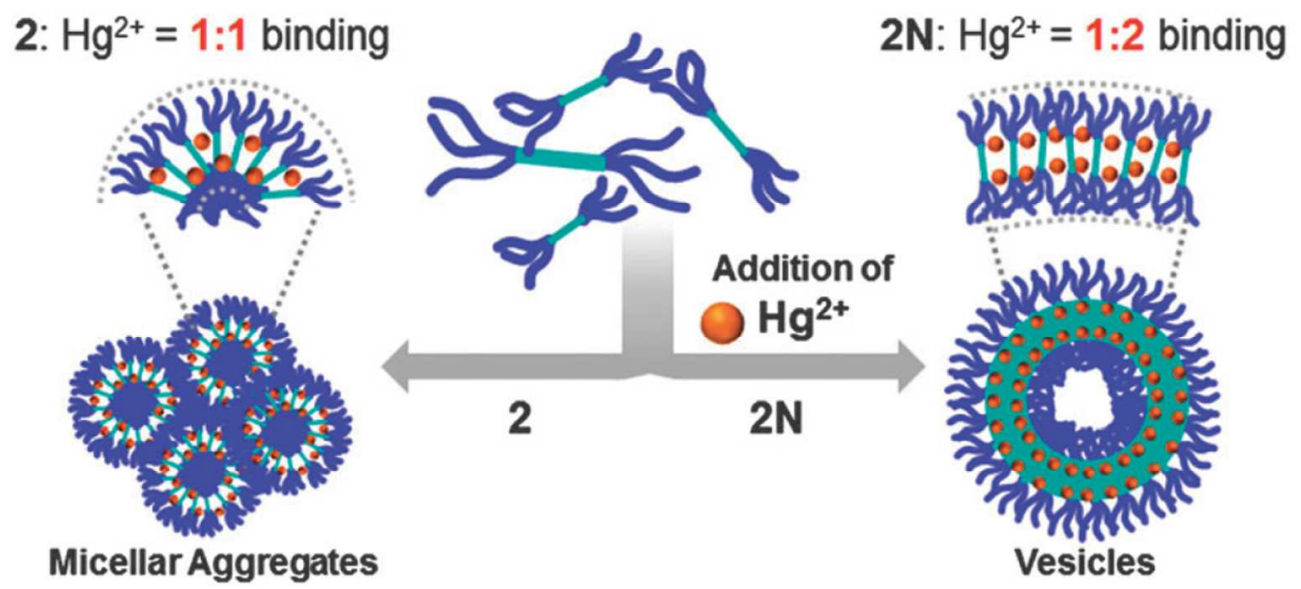

$292 \times 150 \mathrm{~mm}(96 \times 96$ DPI) 


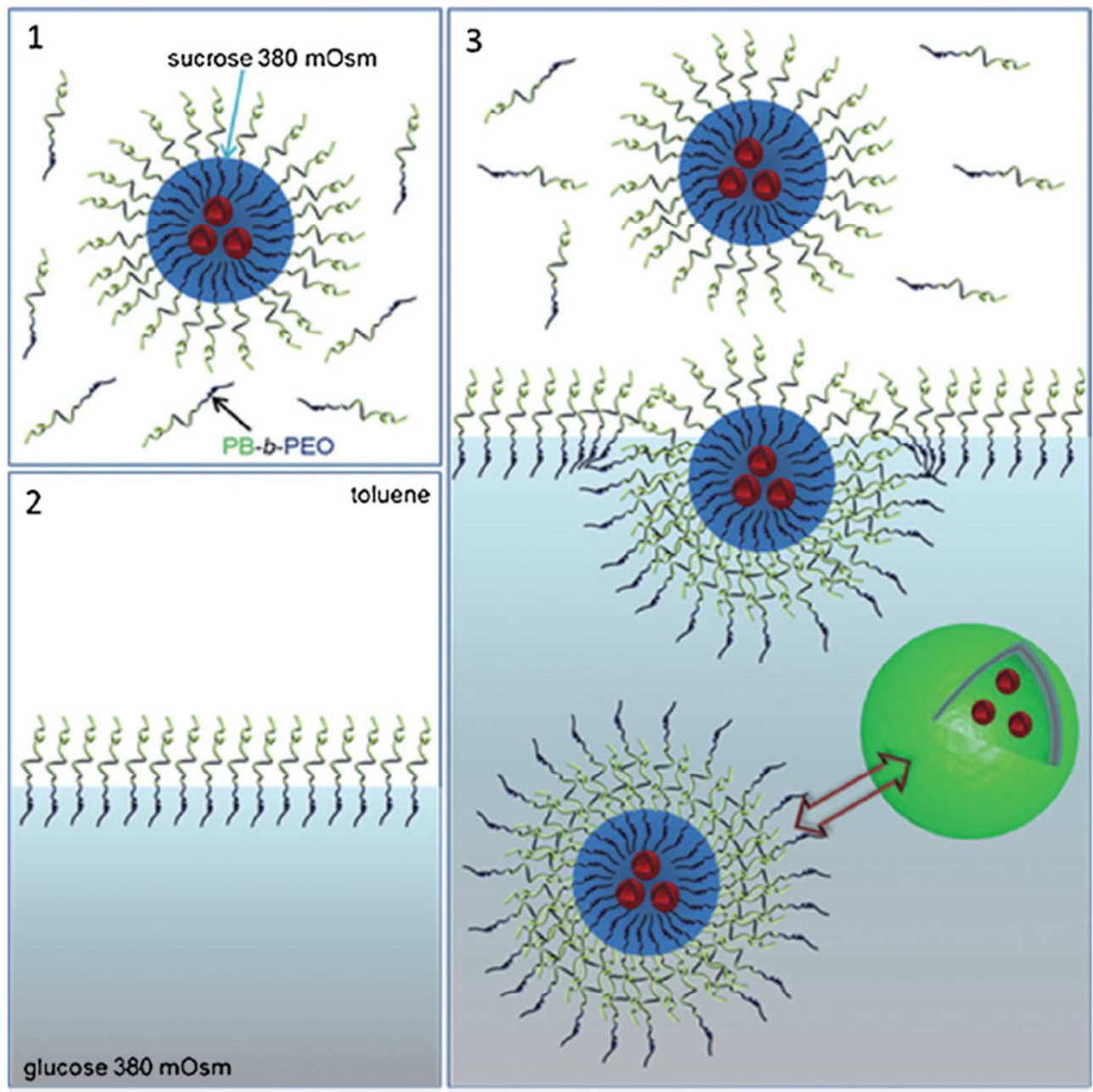

$179 \times 178 \mathrm{~mm}(96 \times 96 \mathrm{DPI})$ 

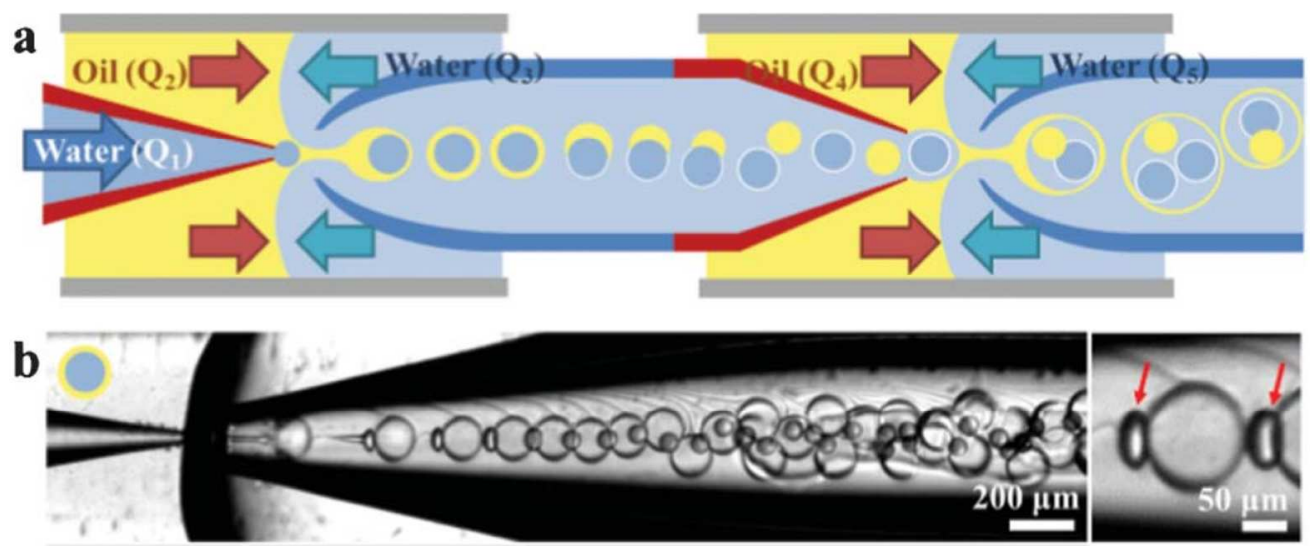

$332 \times 138 \mathrm{~mm}(96 \times 96$ DPI) 


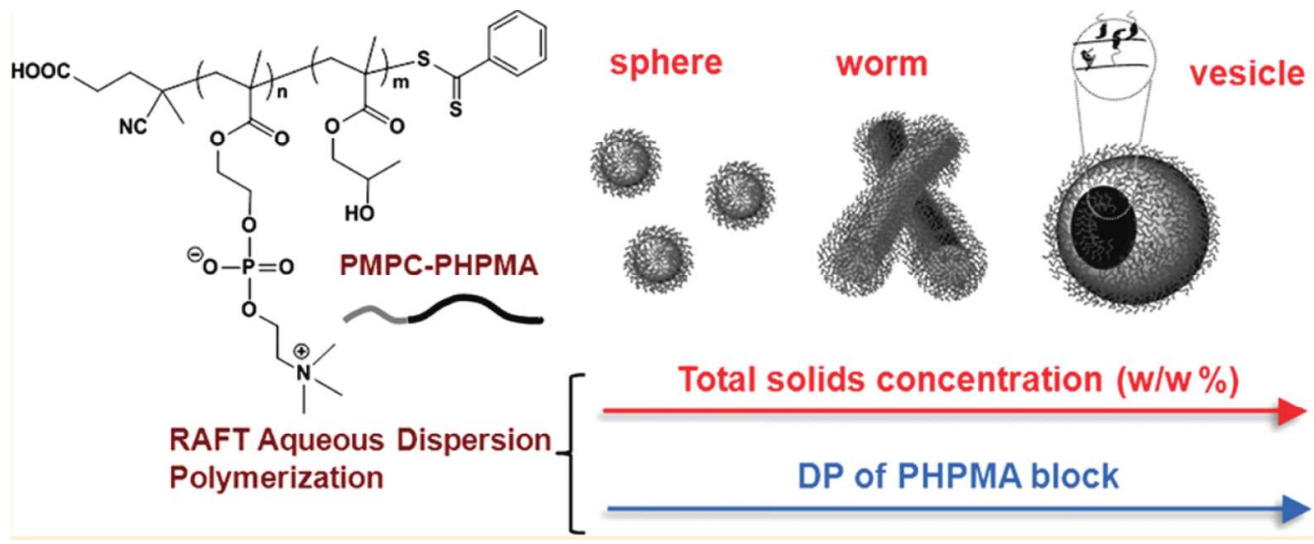

$339 \times 137 m m(96 \times 96$ DPI) 

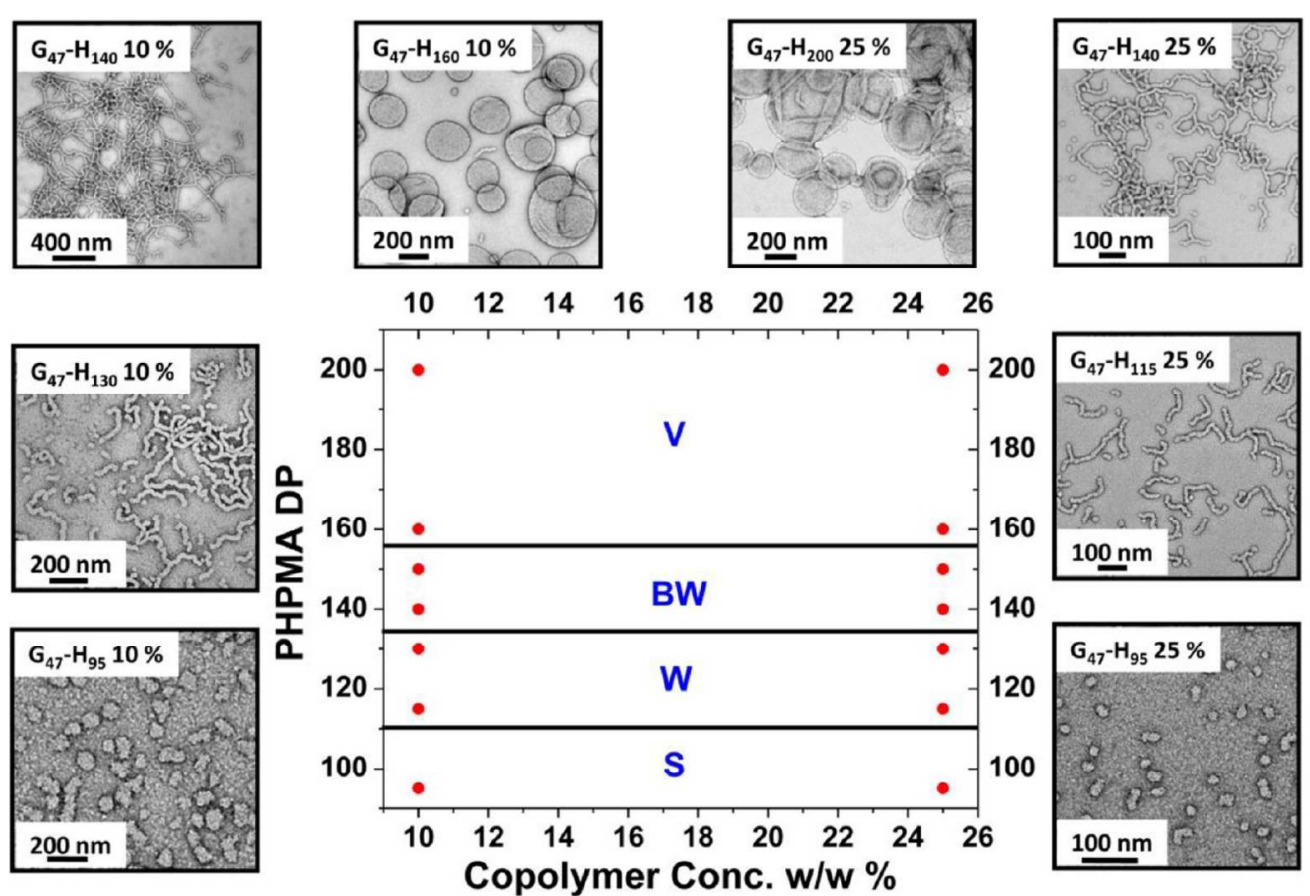

$566 \times 382 \mathrm{~mm}(96 \times 96 \mathrm{DPI})$ 
(A)
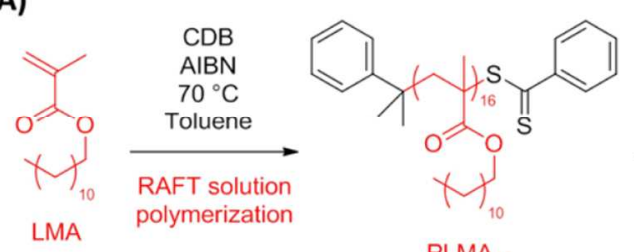

PLMA $_{16}$

(B)

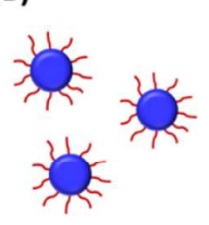

Spheres

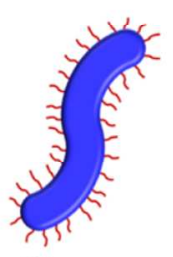

Worms

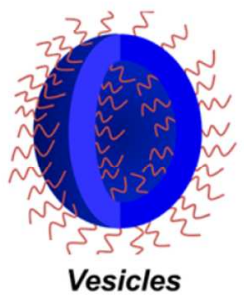

(C)

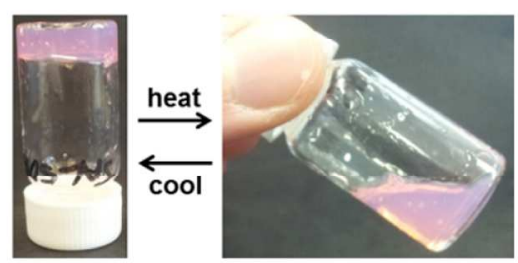

$\mathrm{PLMA}_{16}-\mathrm{PBzMA}_{37}$ in $\boldsymbol{n}$-dodecane

$338 \times 177 \mathrm{~mm}(96 \times 96 \mathrm{DPI})$ 


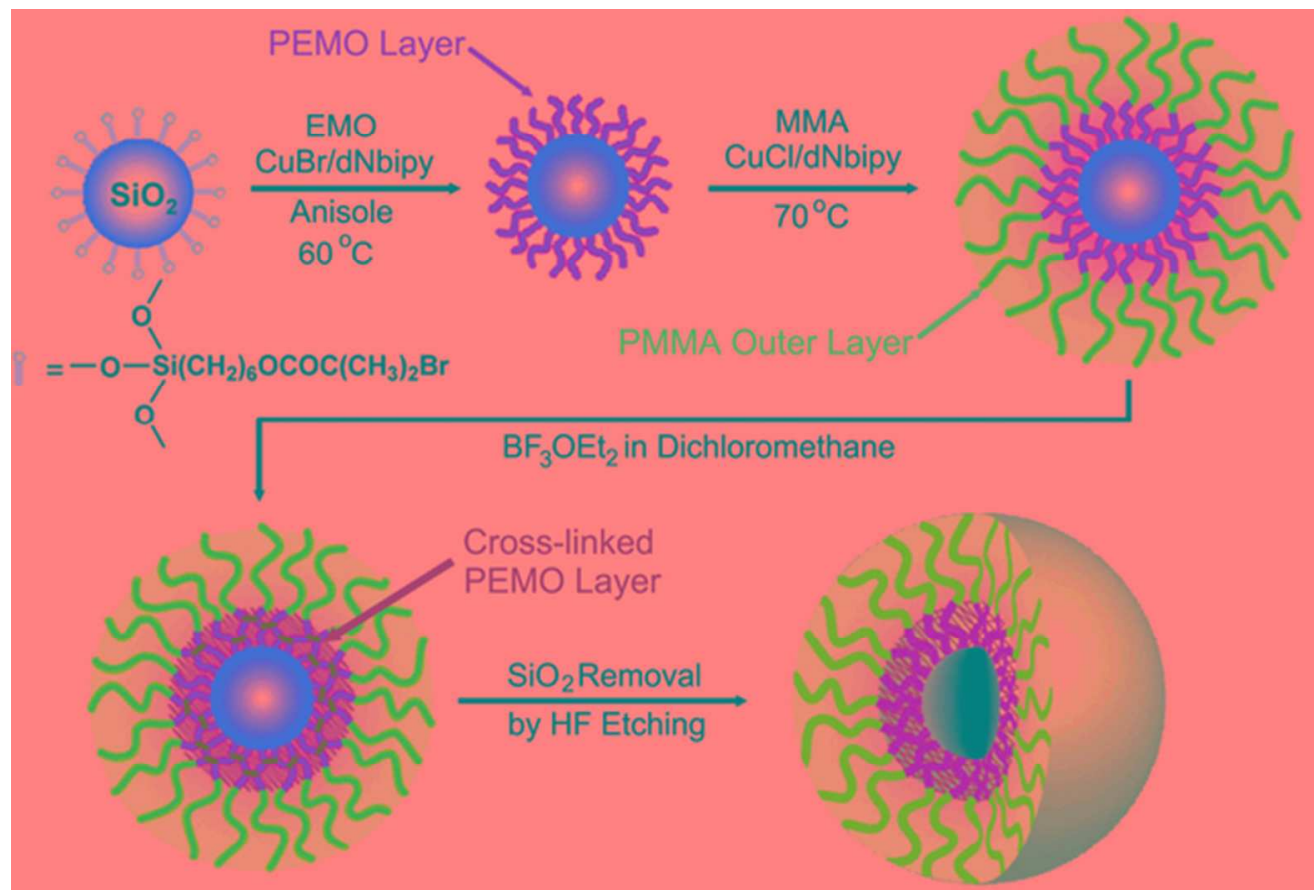

$54 \times 36 \mathrm{~mm}(300 \times 300 \mathrm{DPI})$ 


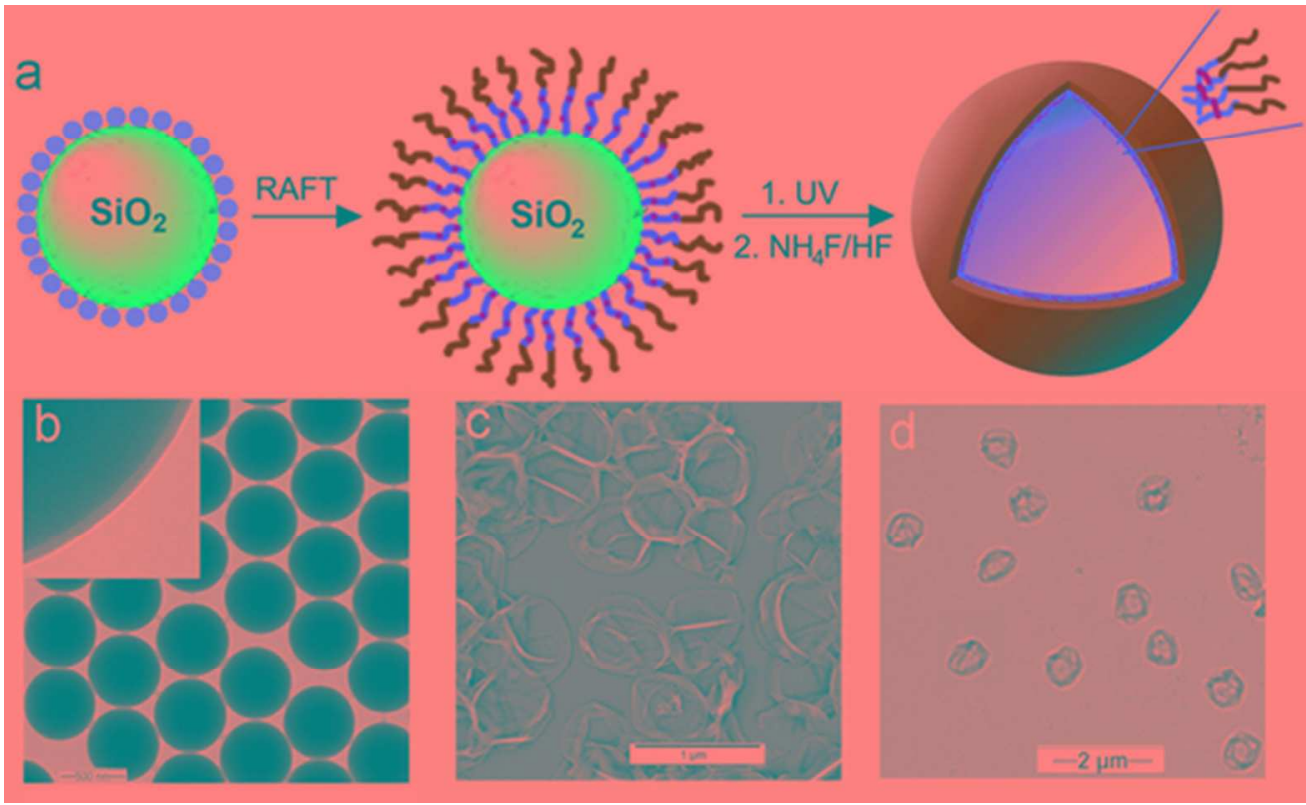

$49 \times 30 \mathrm{~mm}(300 \times 300$ DPI $)$ 


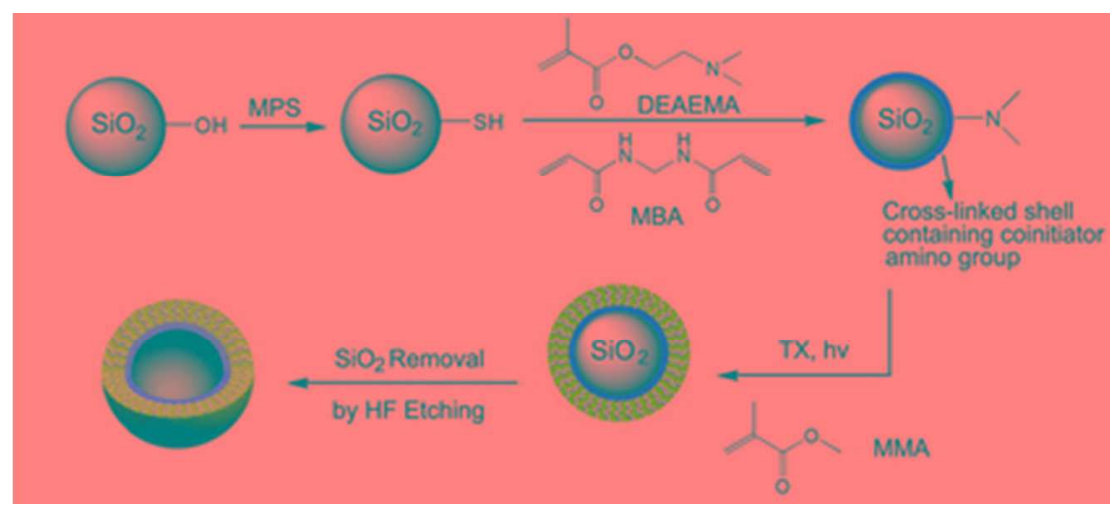

$35 \times 15 \mathrm{~mm}(300 \times 300$ DPI $)$ 


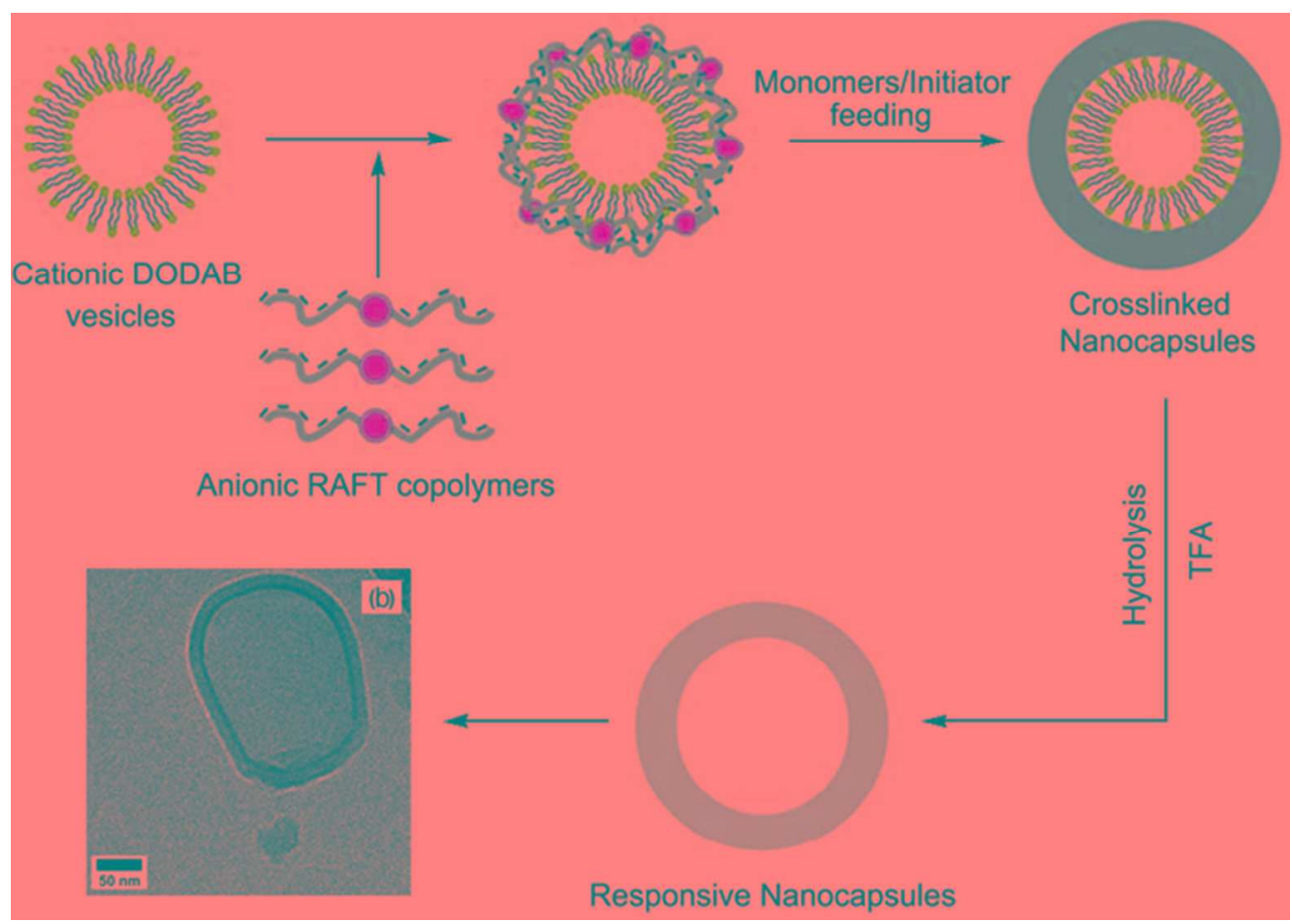

$56 \times 40 \mathrm{~mm}(300 \times 300 \mathrm{DPI})$ 


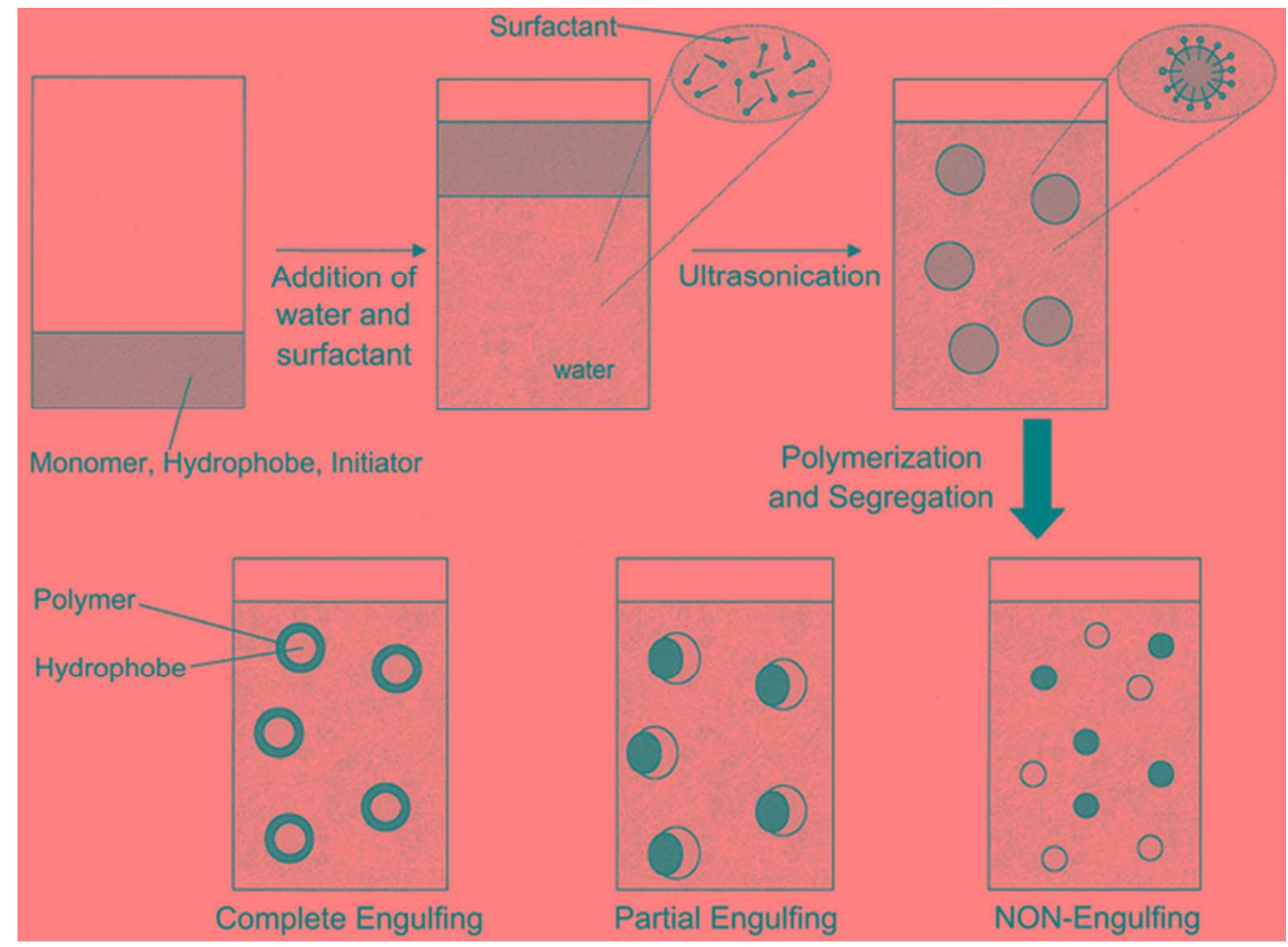

$59 \times 43 \mathrm{~mm}(300 \times 300$ DPI $)$ 


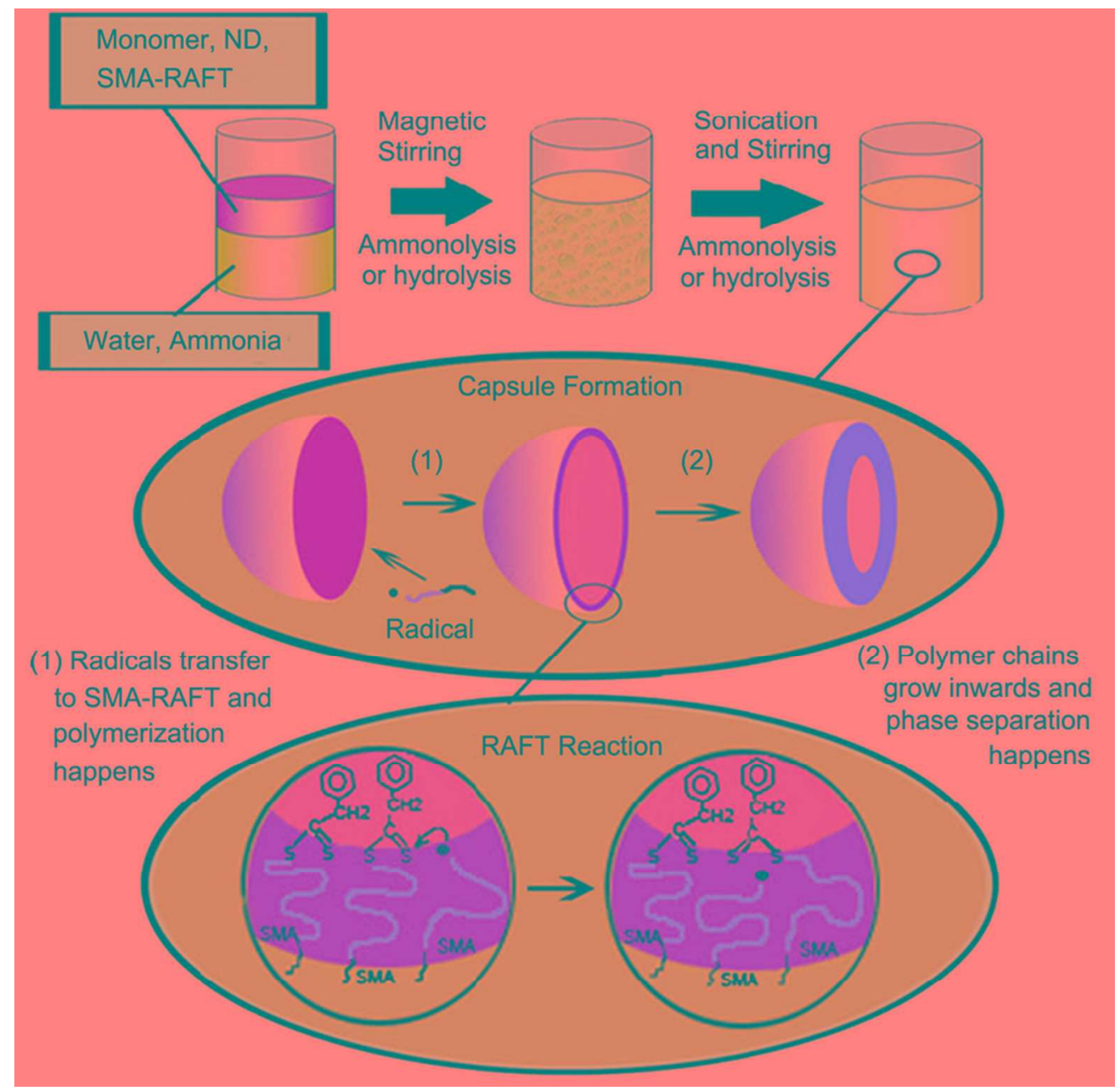

$78 \times 76 \mathrm{~mm}(300 \times 300 \mathrm{DPI})$ 


1
2
3
4
5
6
7
8
9
10
11
12
13
14
15
16
17
18
19
20
21
22
23
24
25
26
27
28
29
30
31
32
33
34
35
36
37
38
39
40
41
42
43
44
45
46
47
50
58
59
50
52
53
50

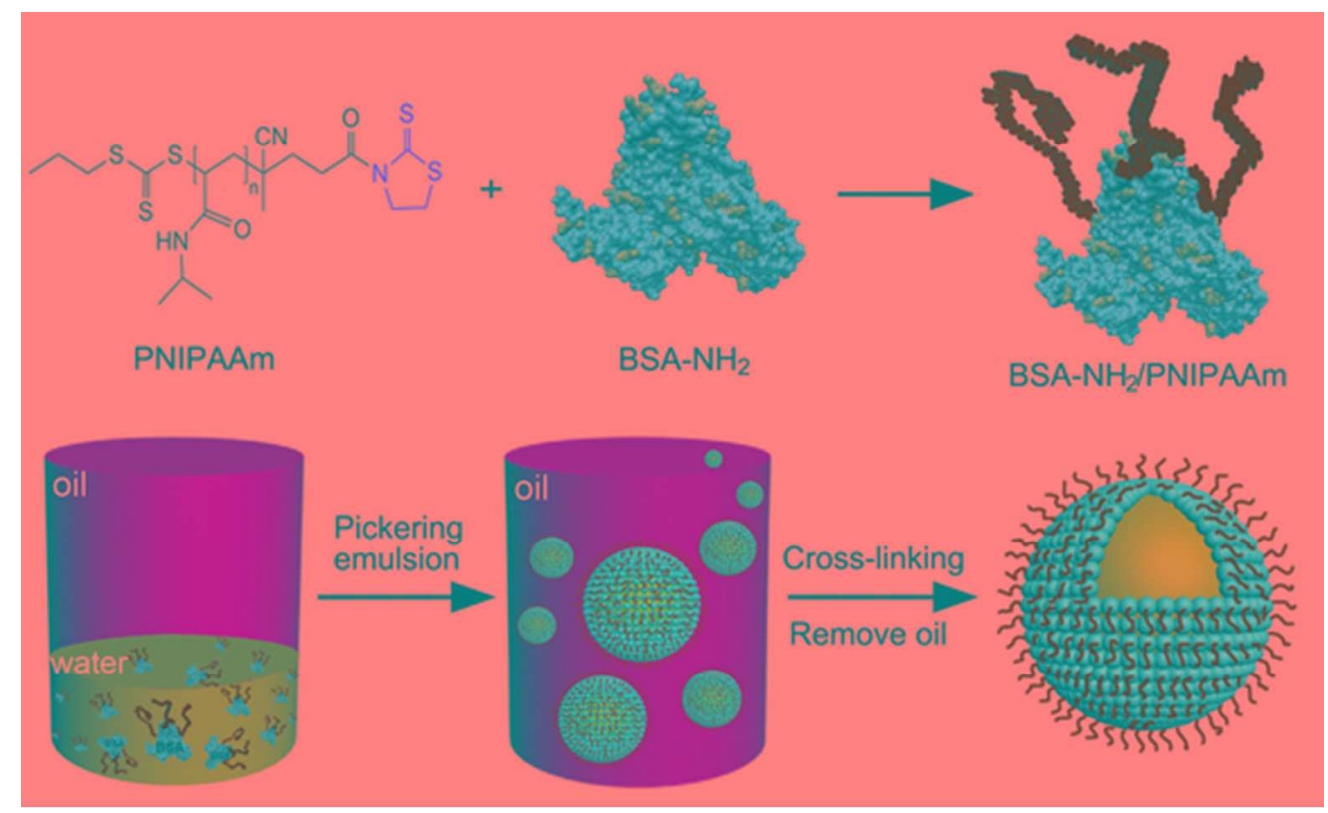

$48 \times 29 \mathrm{~mm}(300 \times 300 \mathrm{DPI})$ 
6

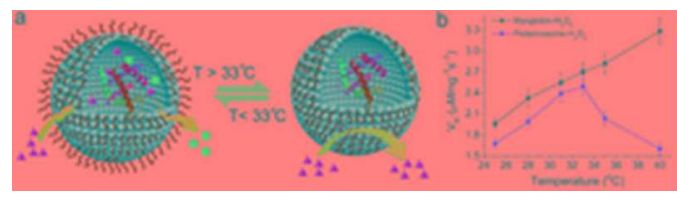

$21 \times 5 \mathrm{~mm}(300 \times 300 \mathrm{DPI})$ 
1

2

3

4

5

6

7

8

9

10

11

12

13

14

15

16

17

18

19

20

21

22

23

24

25

26

27

28

29

30

31

32

33

34

35

36

37

38

39

40

41

42

43

44

45

46

47

48

49

50

51

52

53

54

55

56

57

58

59

60

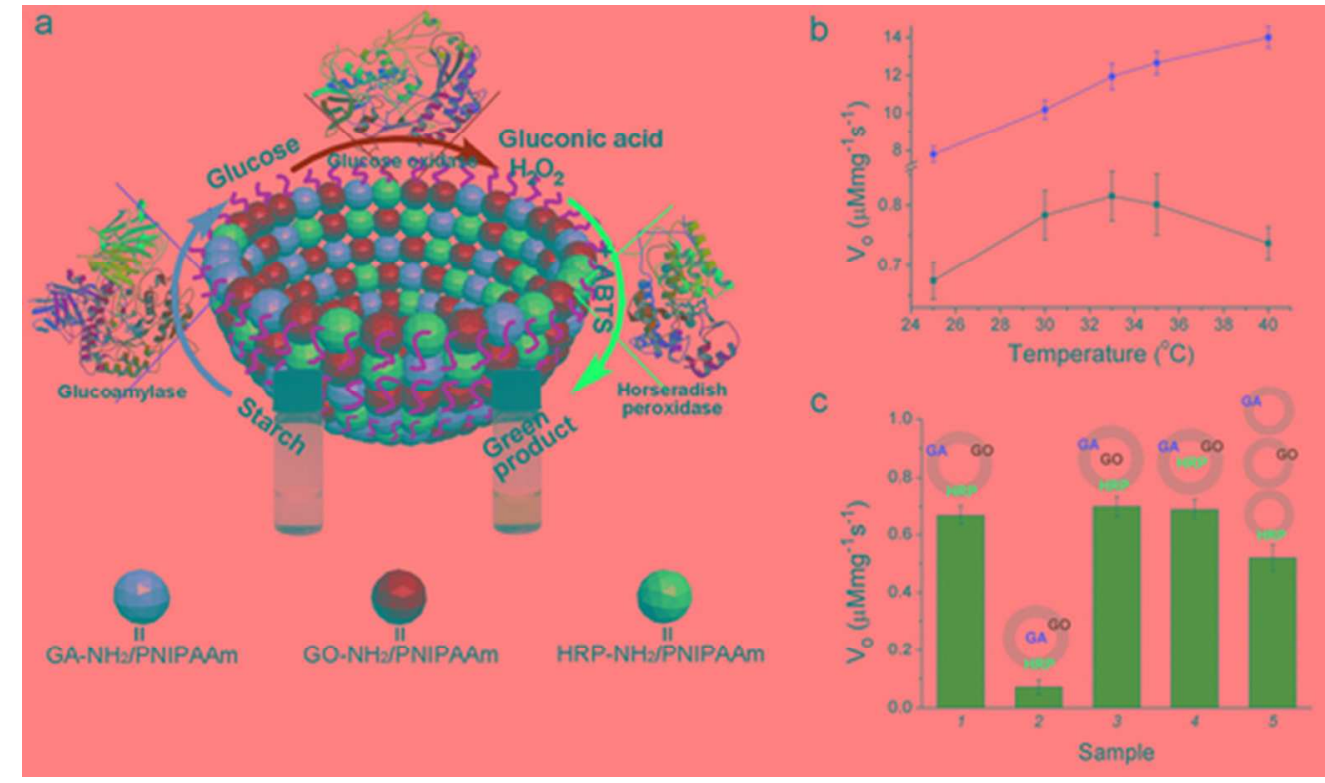

$47 \times 28 \mathrm{~mm}(300 \times 300$ DPI $)$ 

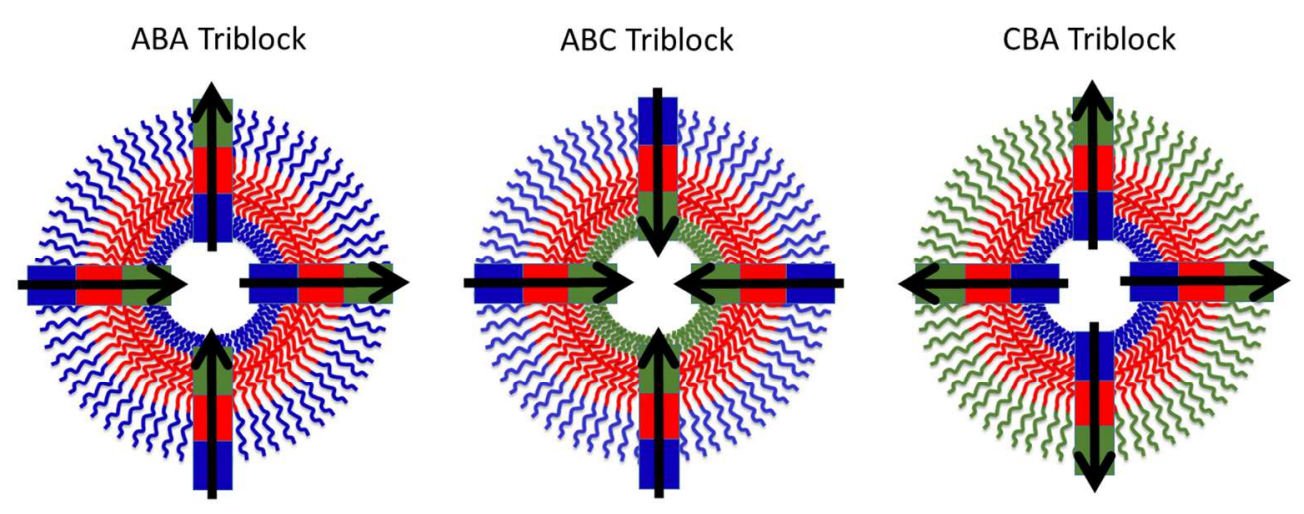

$370 \times 139 m m(96 \times 96$ DPI) 


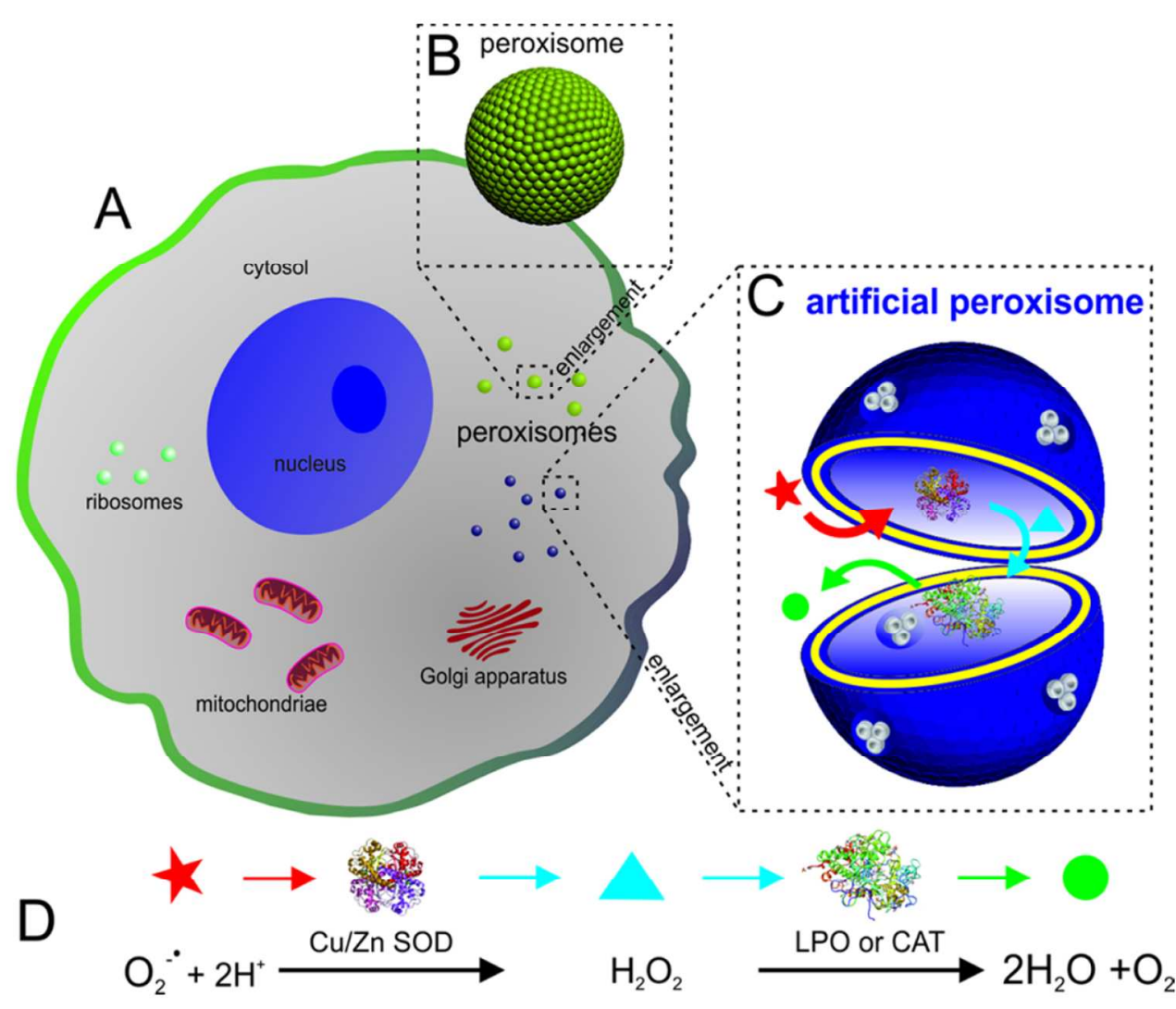

$352 \times 287 \mathrm{~mm}(96 \times 96$ DPI) 

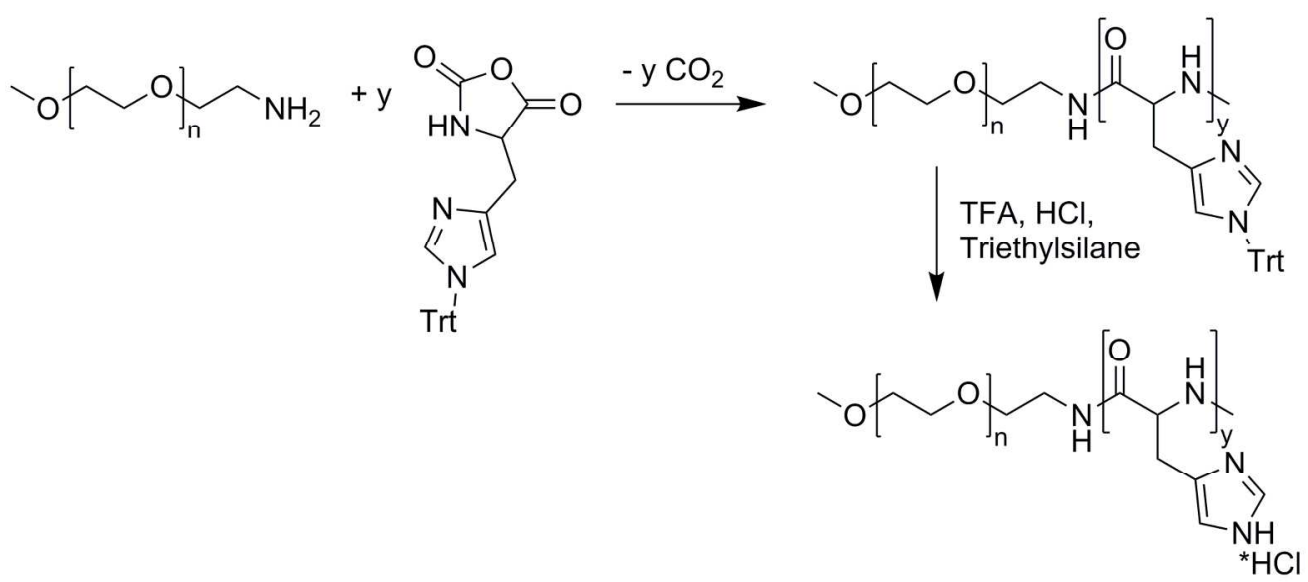

$179 \times 79 \mathrm{~mm}(300 \times 300$ DPI) 


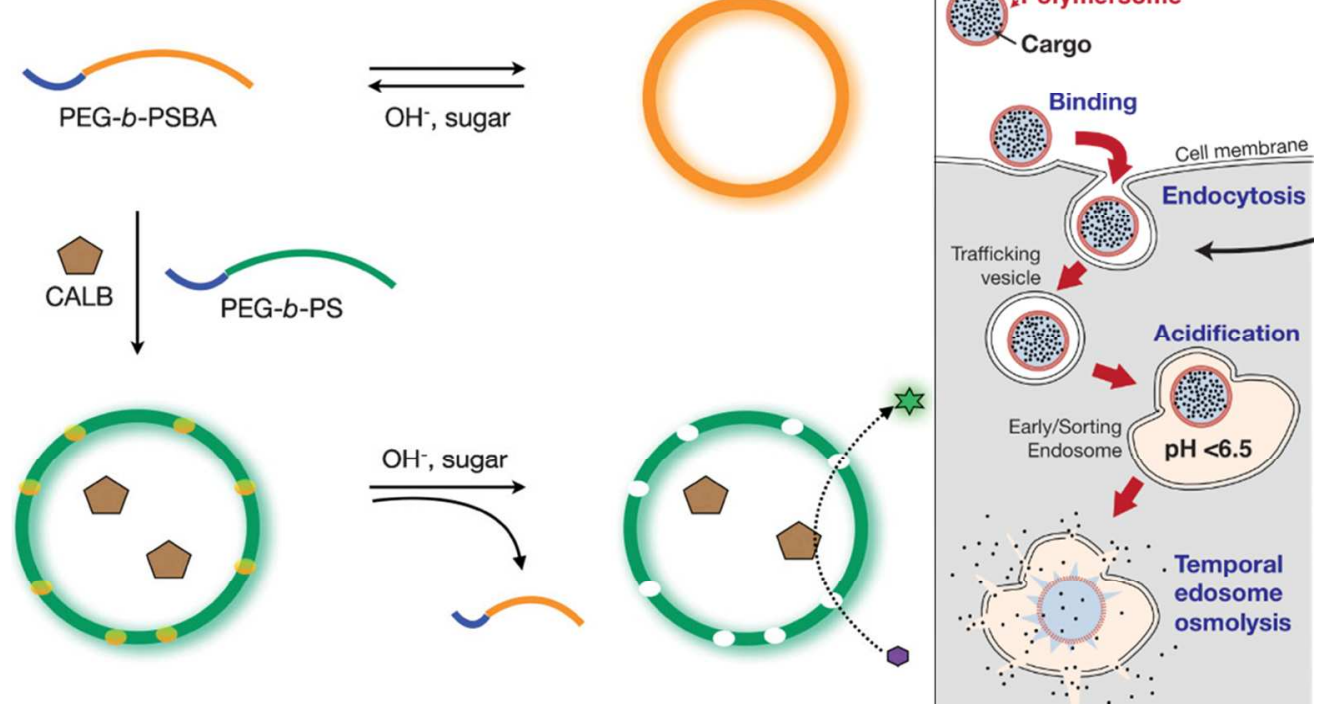

$338 \times 190 \mathrm{~mm}(96 \times 96$ DPI) 

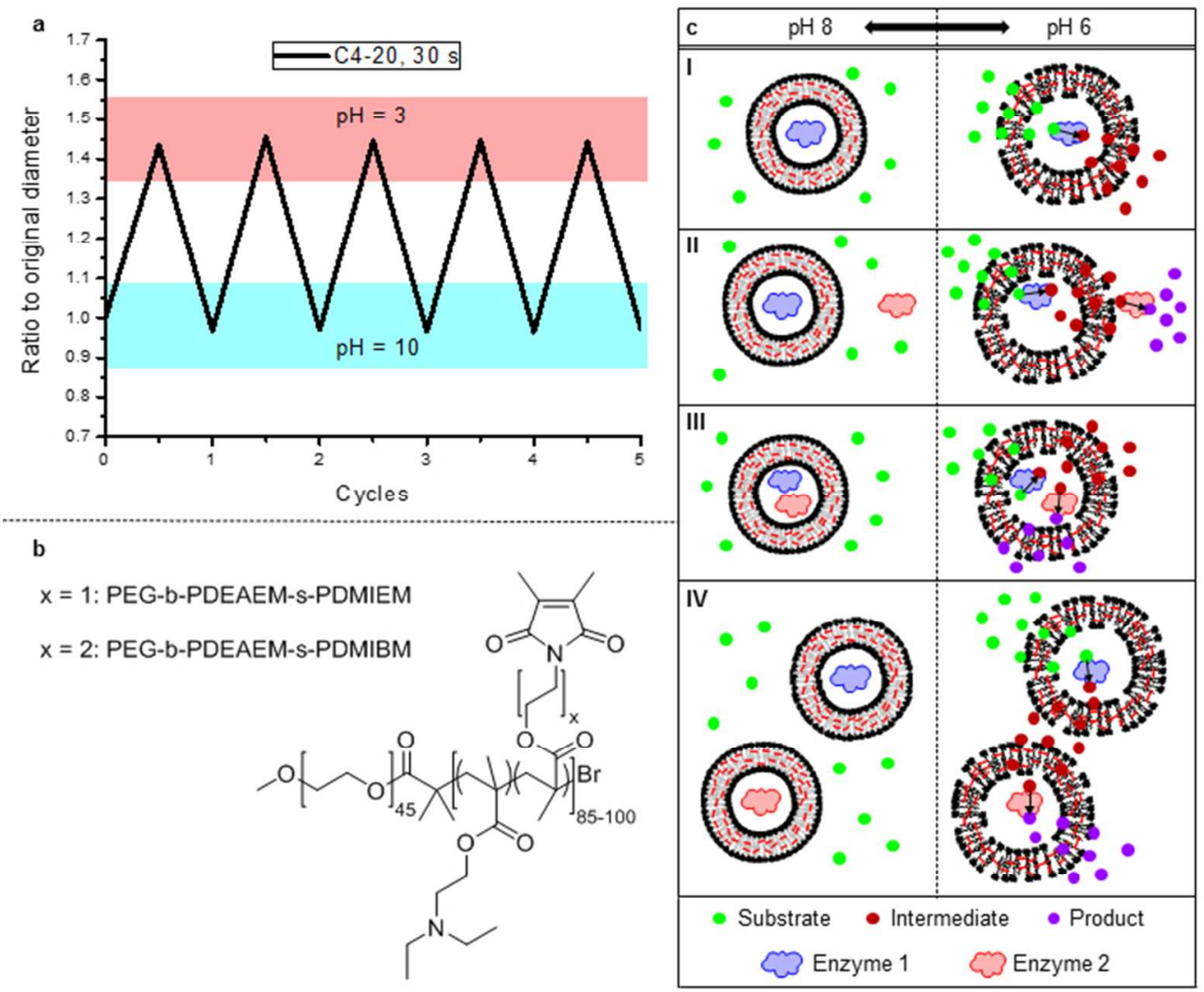

$190 \times 160 \mathrm{~mm}(96 \times 96 \mathrm{DPI})$ 
(a)

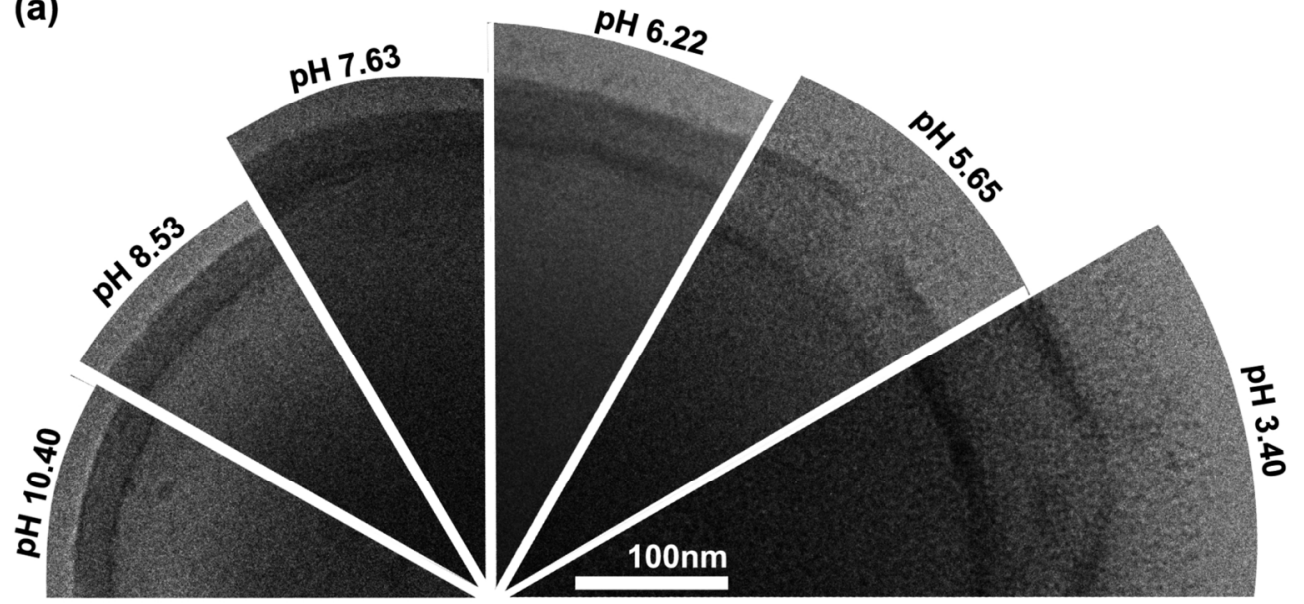

(b)

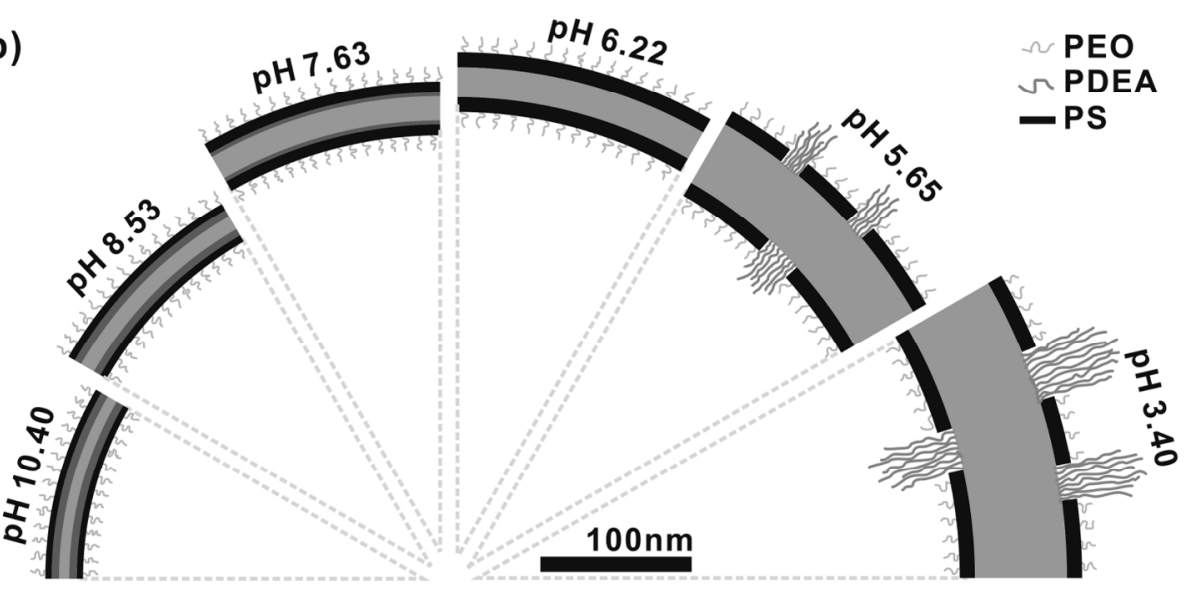

688x666mm (96 x 96 DPI) 


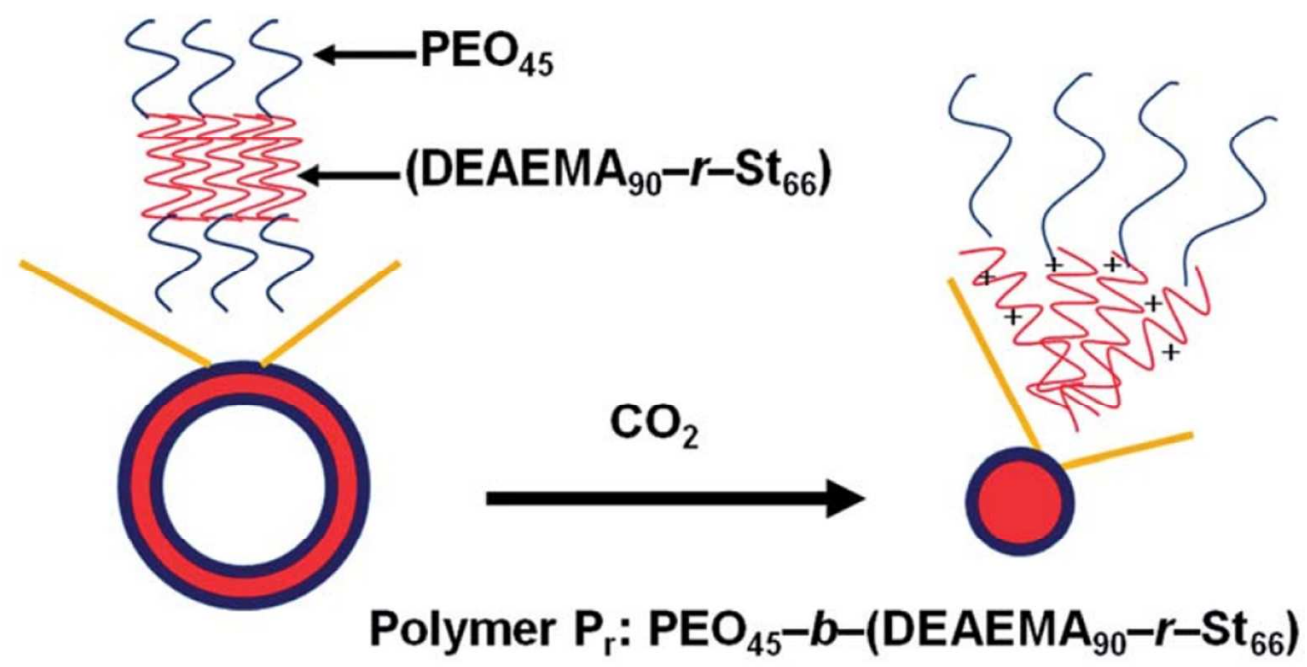

$271 \times 139 m m(96 \times 96$ DPI) 

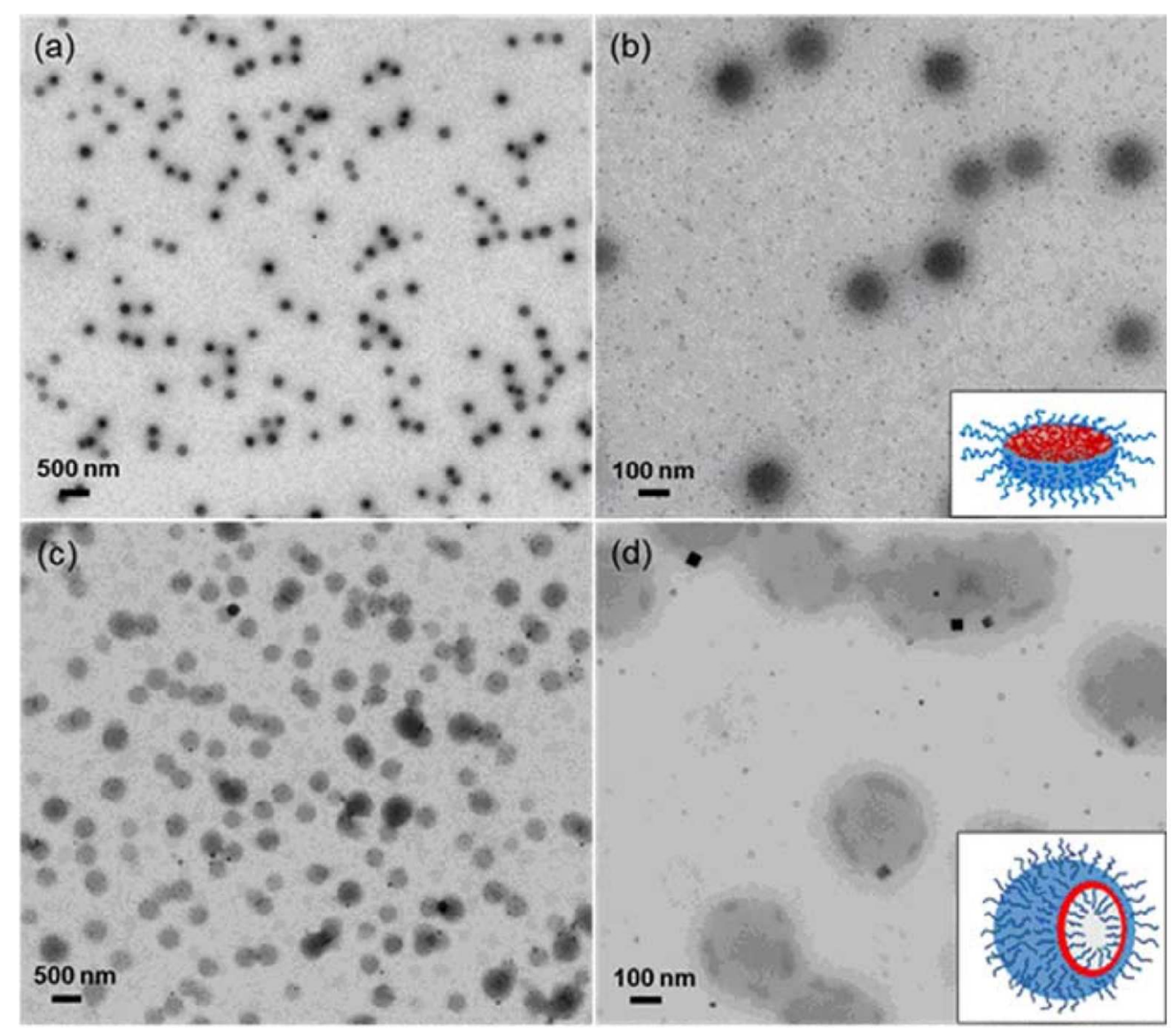

$339 \times 299 m m(96 \times 96$ DPI $)$ 

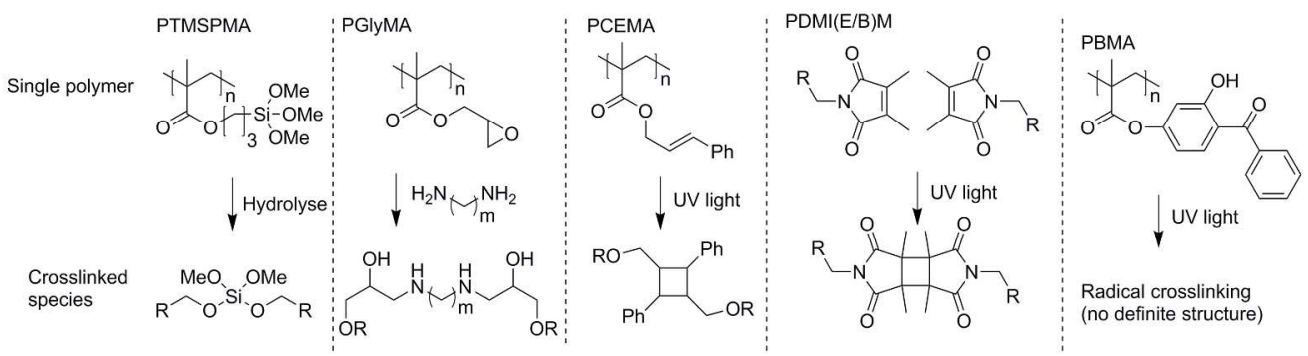

$240 \times 64 \mathrm{~mm}(300 \times 300 \mathrm{DPI})$ 


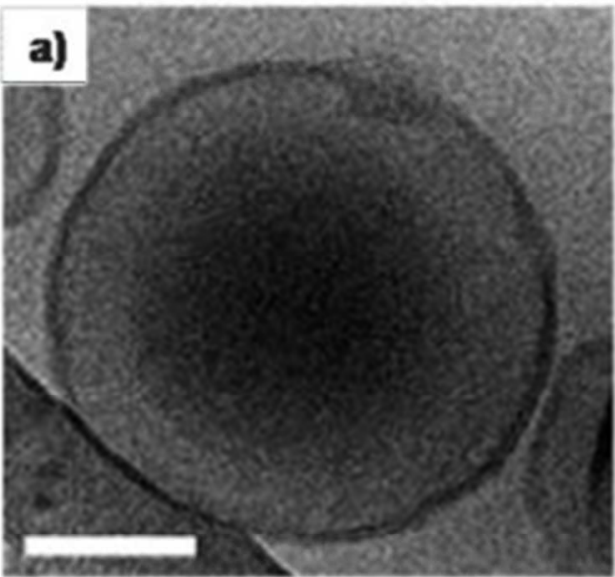

\section{b)}
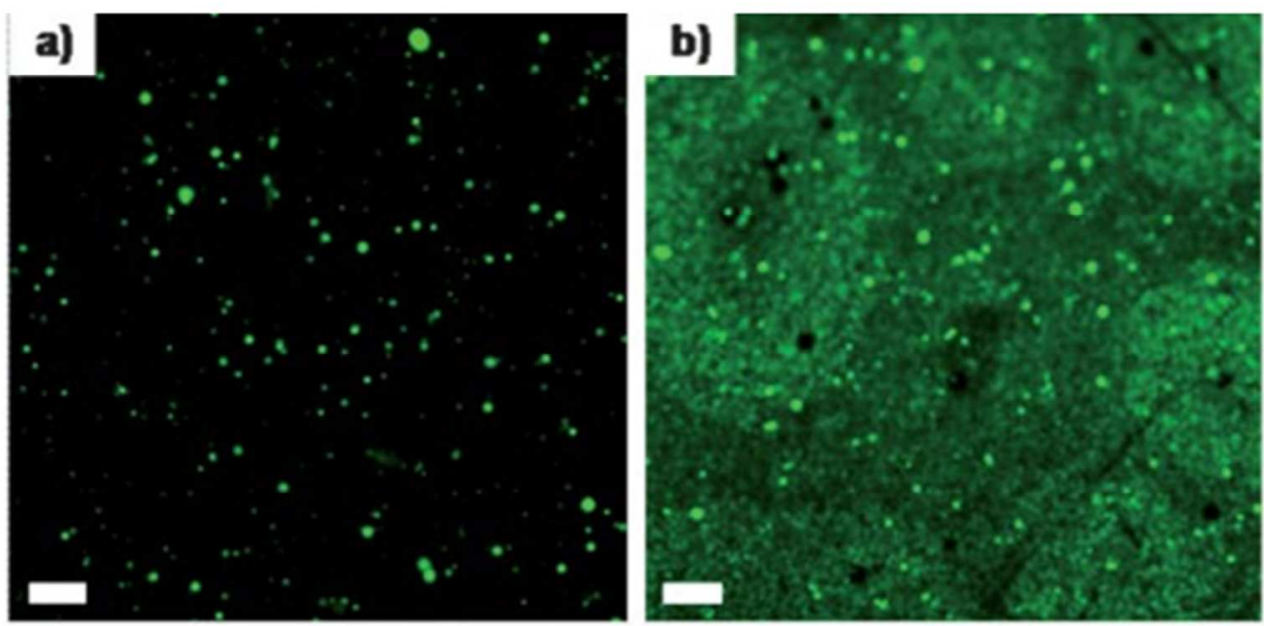

166x160mm (96 x 96 DPI) 

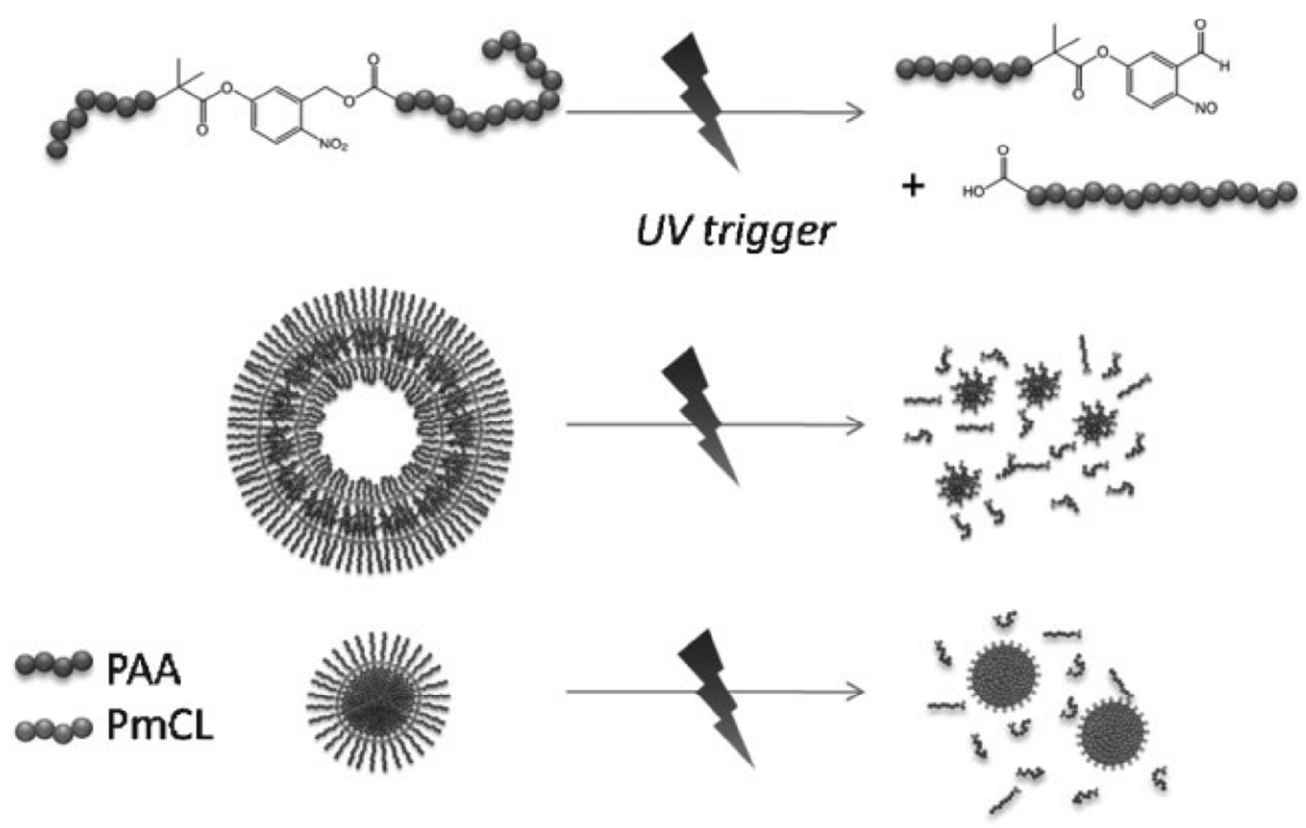

$331 \times 209 \mathrm{~mm}(96 \times 96$ DPI $)$ 


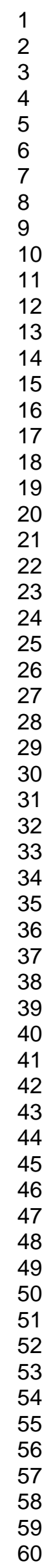
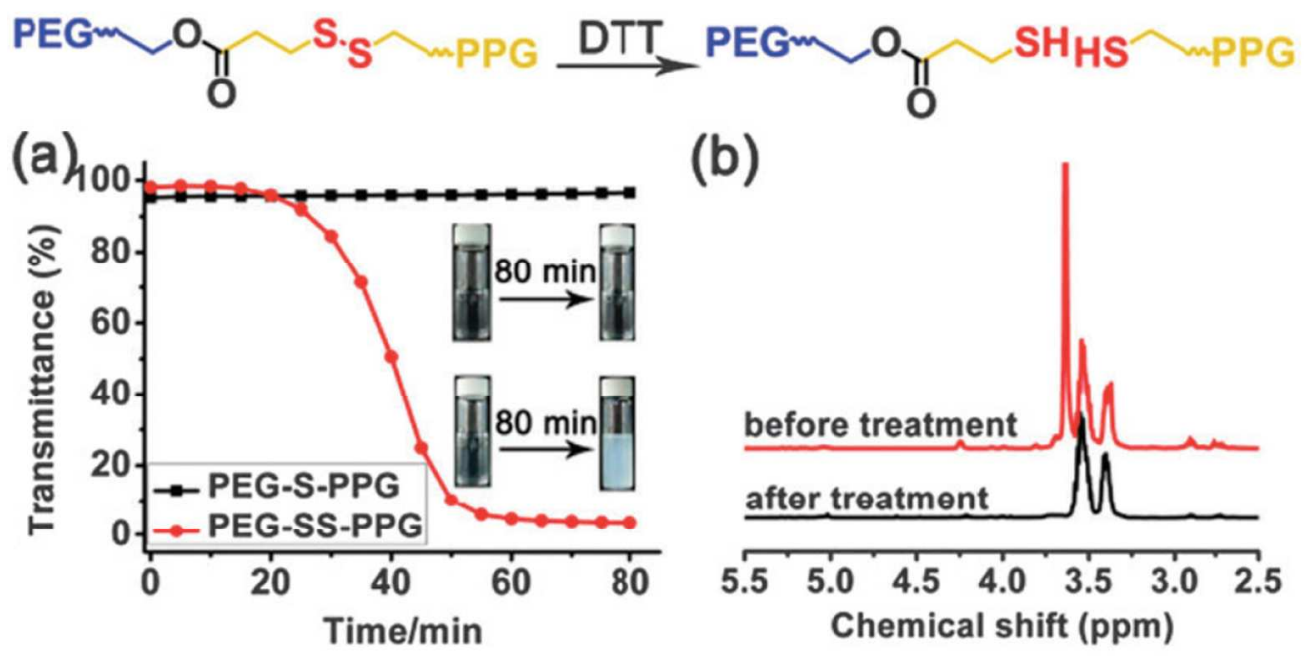

(b)

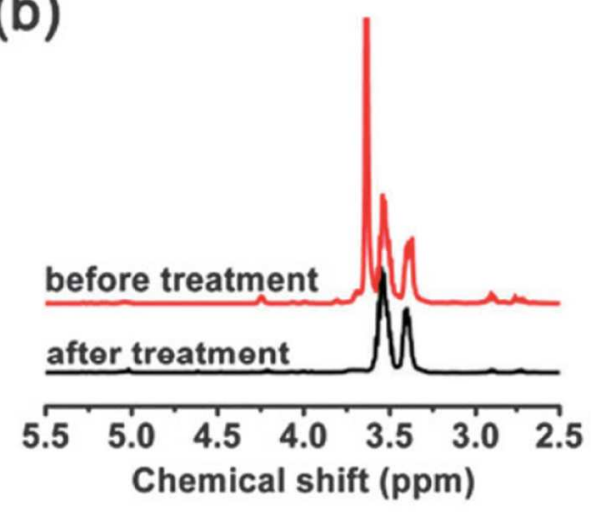

$282 \times 144 m m(96 \times 96$ DPI) 


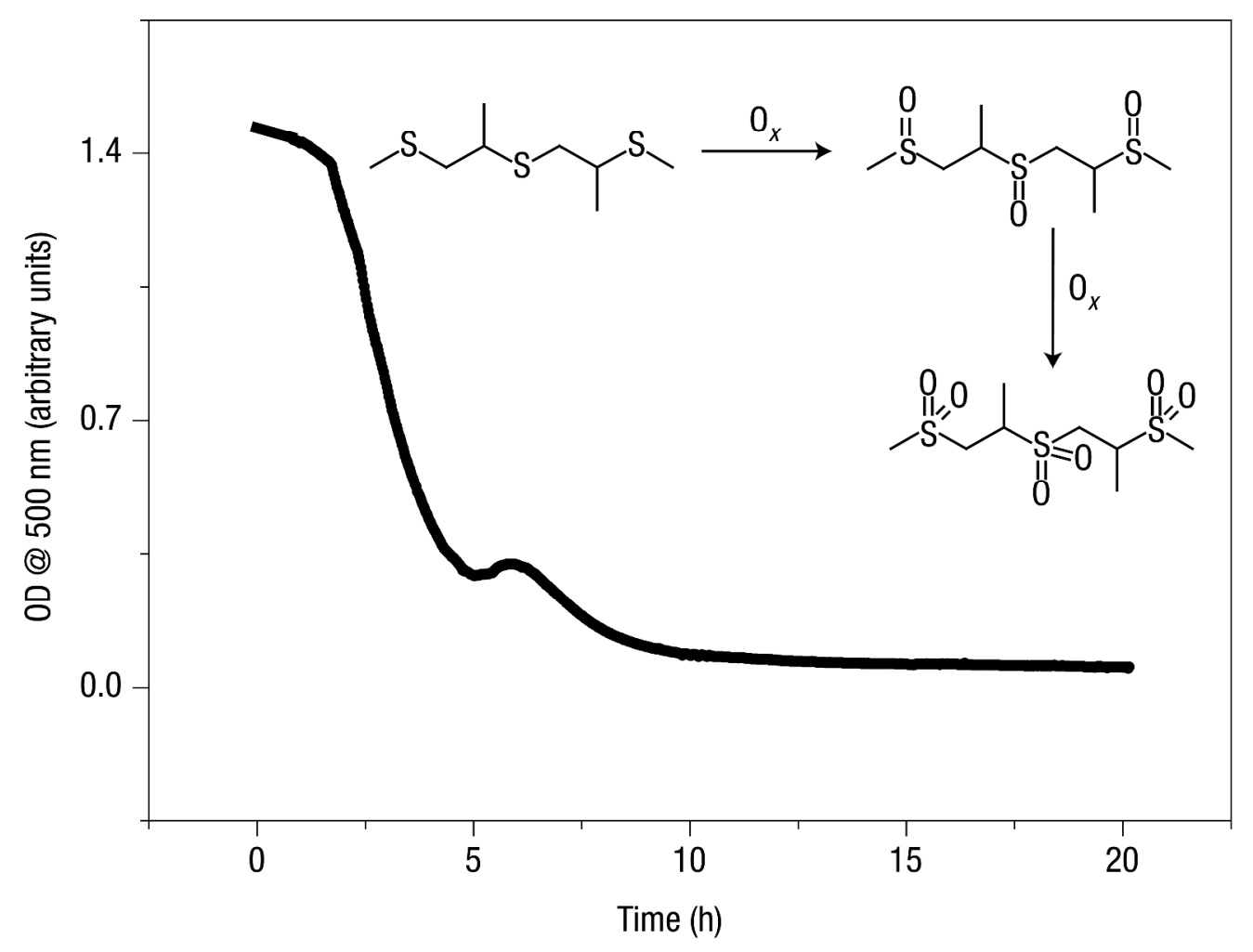

$1359 \times 1050 \mathrm{~mm}(96 \times 96 \mathrm{DPI})$ 


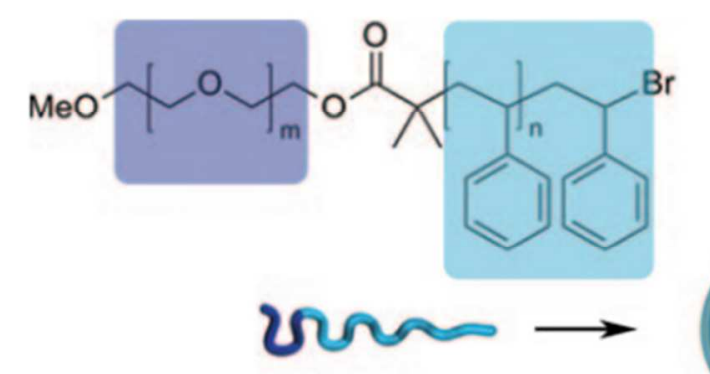

$\mathrm{PEG}_{44-b-\mathrm{PS}_{182-292}}$
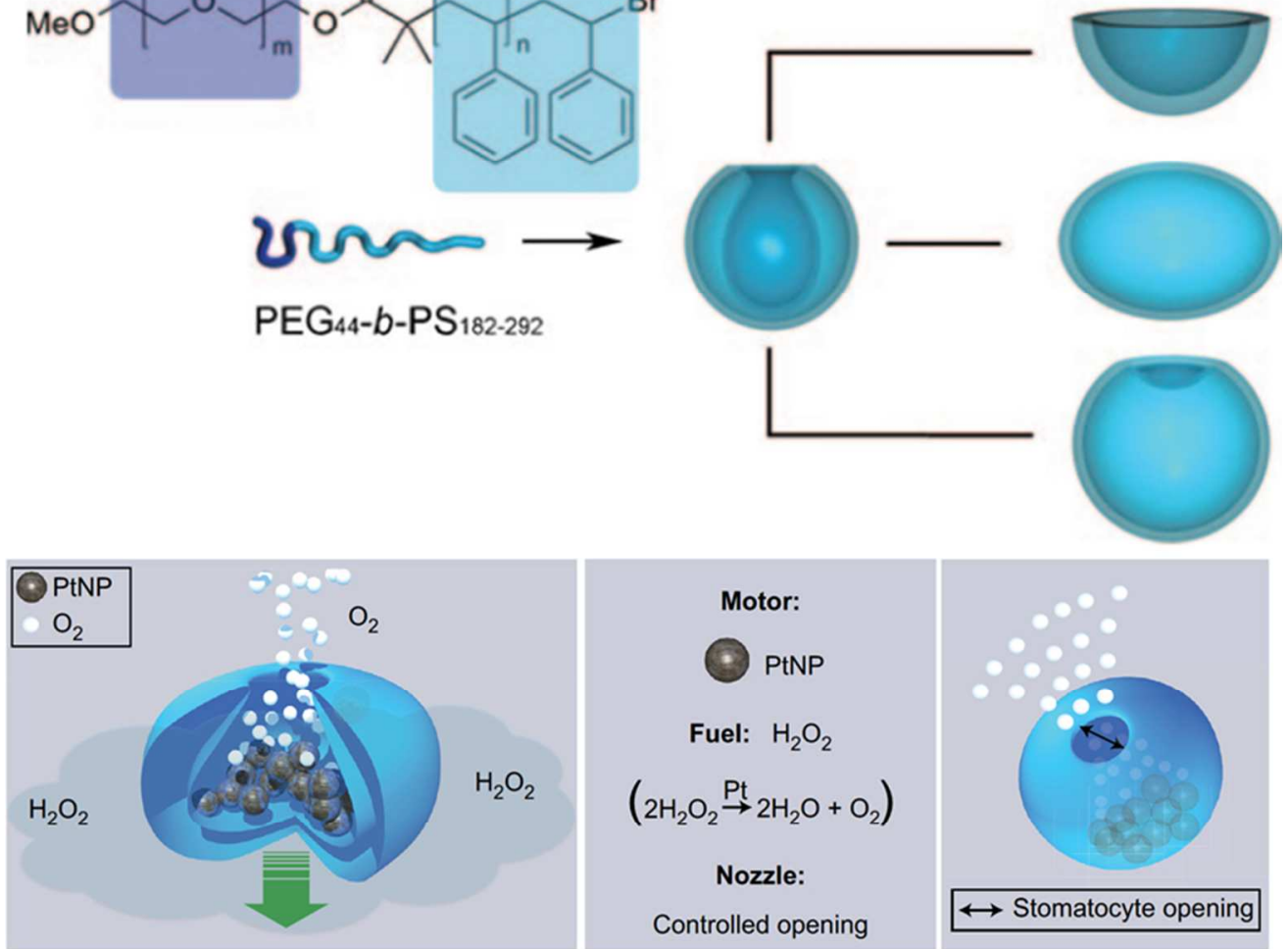

$229 \times 190 \mathrm{~mm}(96 \times 96 \mathrm{DPI})$ 


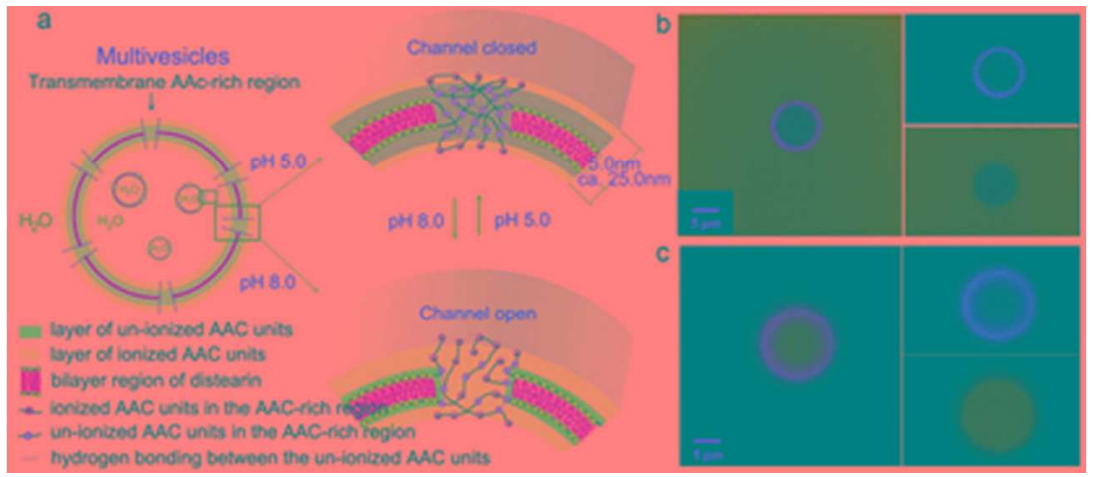

$35 \times 15 \mathrm{~mm}(300 \times 300$ DPI $)$ 
1

2

3

4

5

6

7

8

9

10

11

12

13

14

15

16

17

18

19

20

21

22

23

24

25

26

27

28

29

30

31

32

33

34

35

36

37

38

39

40

41

42

43

44

45

46

47

48

49

50

51

52

53

54

55

56

57

58

59

60

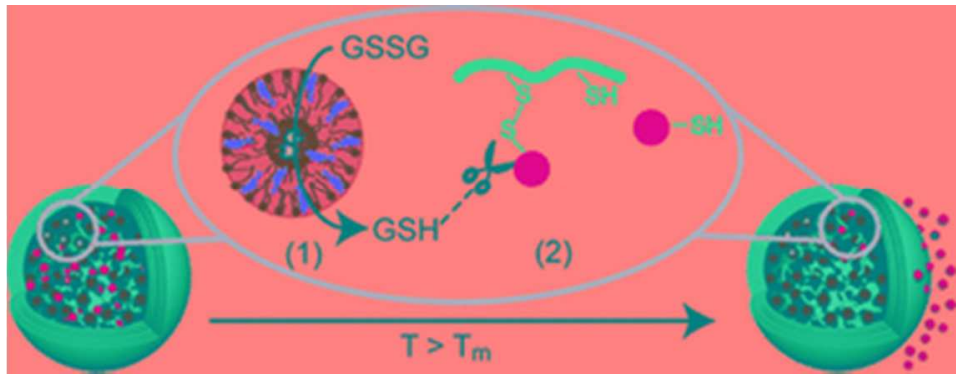

$30 \times 11 \mathrm{~mm}(300 \times 300 \mathrm{DPI})$ 


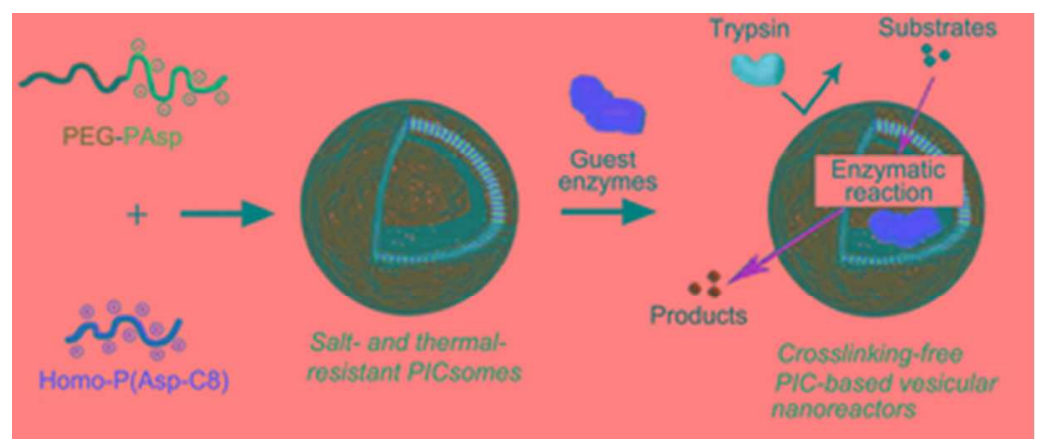

$33 \times 13 \mathrm{~mm}(300 \times 300$ DPI $)$ 


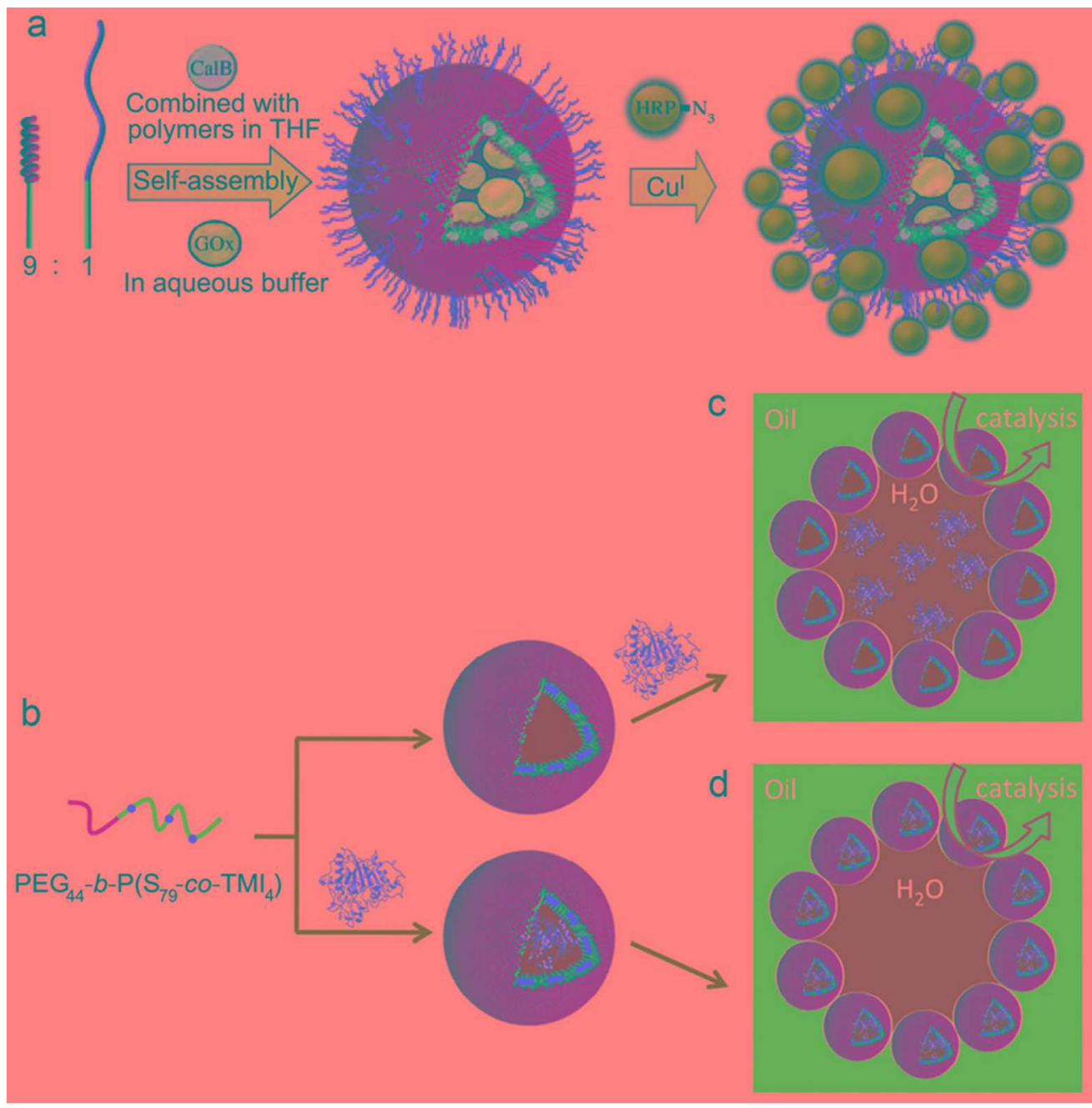

$80 \times 81 \mathrm{~mm}(300 \times 300$ DPI $)$ 


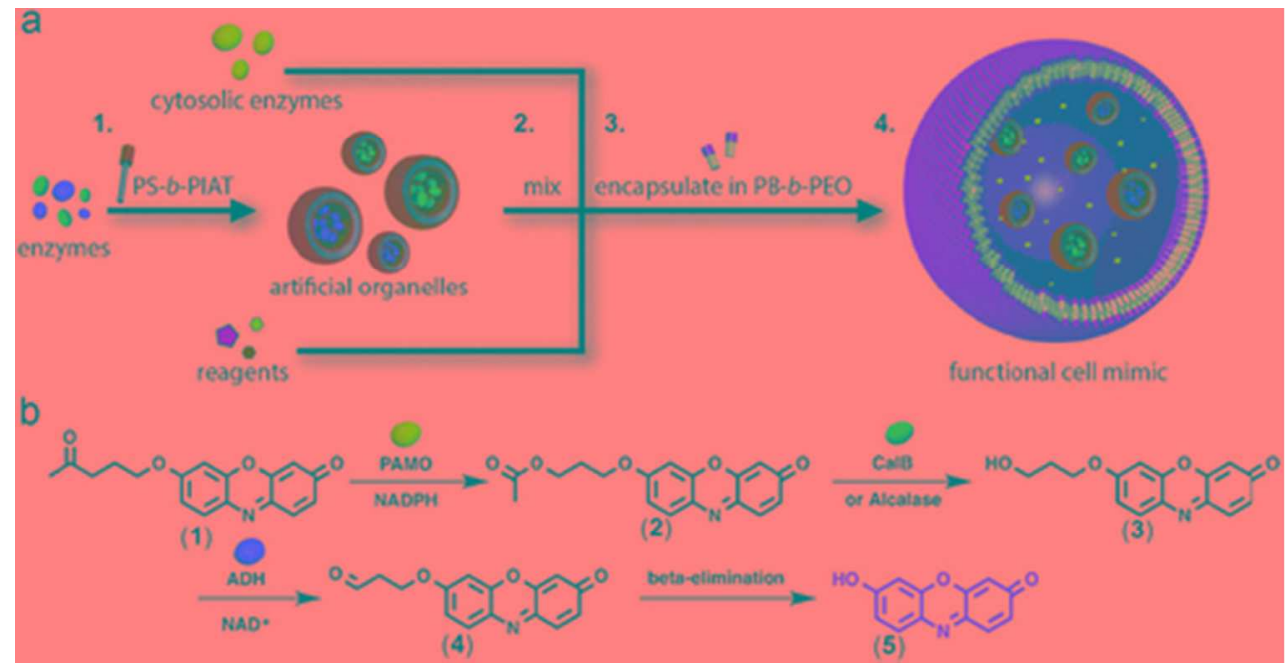

$41 \times 21 \mathrm{~mm}(300 \times 300$ DPI $)$ 


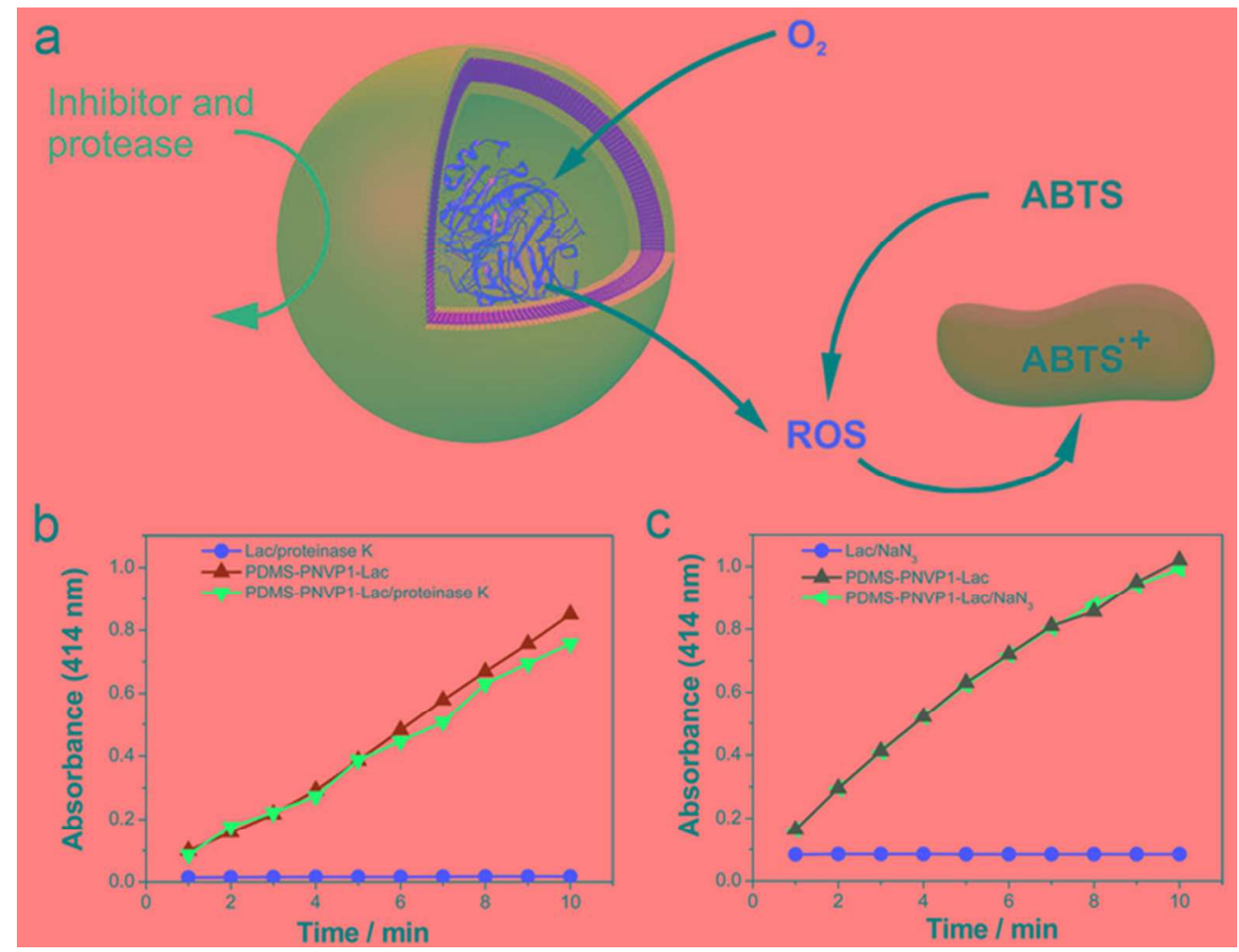

$61 \times 47 \mathrm{~mm}(300 \times 300$ DPI $)$ 


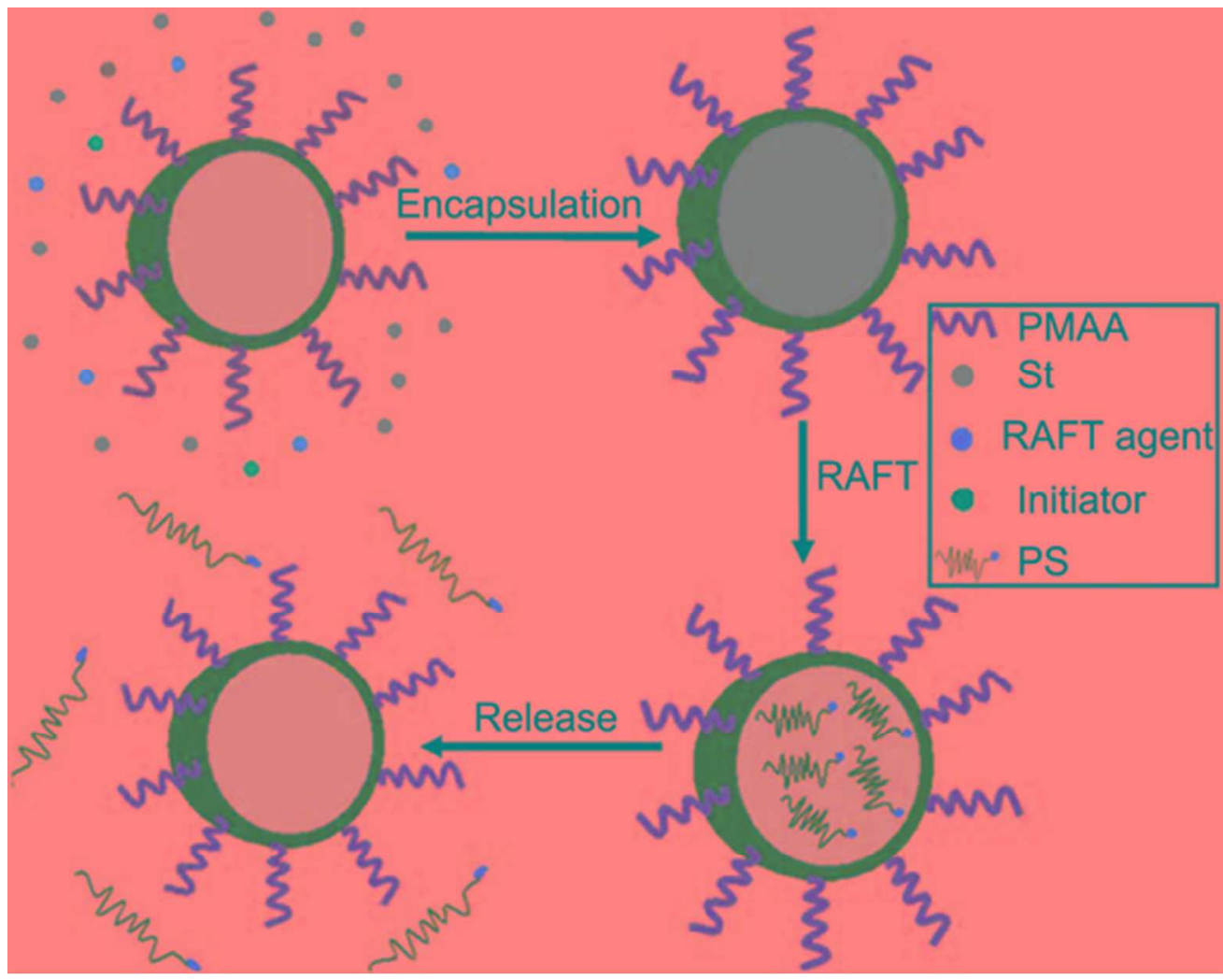

$47 \times 37 \mathrm{~mm}(300 \times 300$ DPI $)$ 


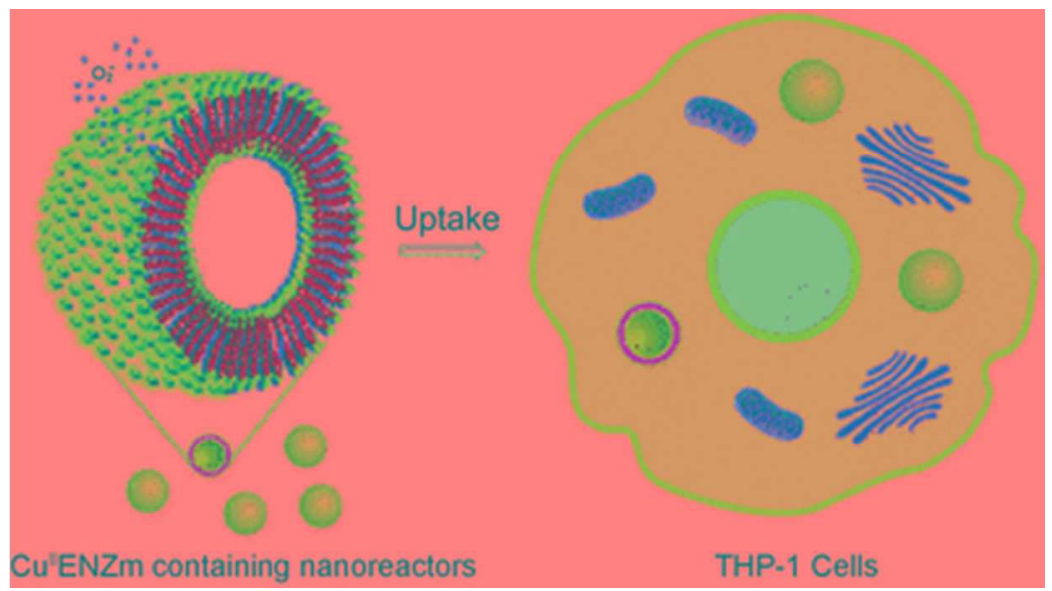

$33 \times 18 \mathrm{~mm}(300 \times 300$ DPI $)$ 


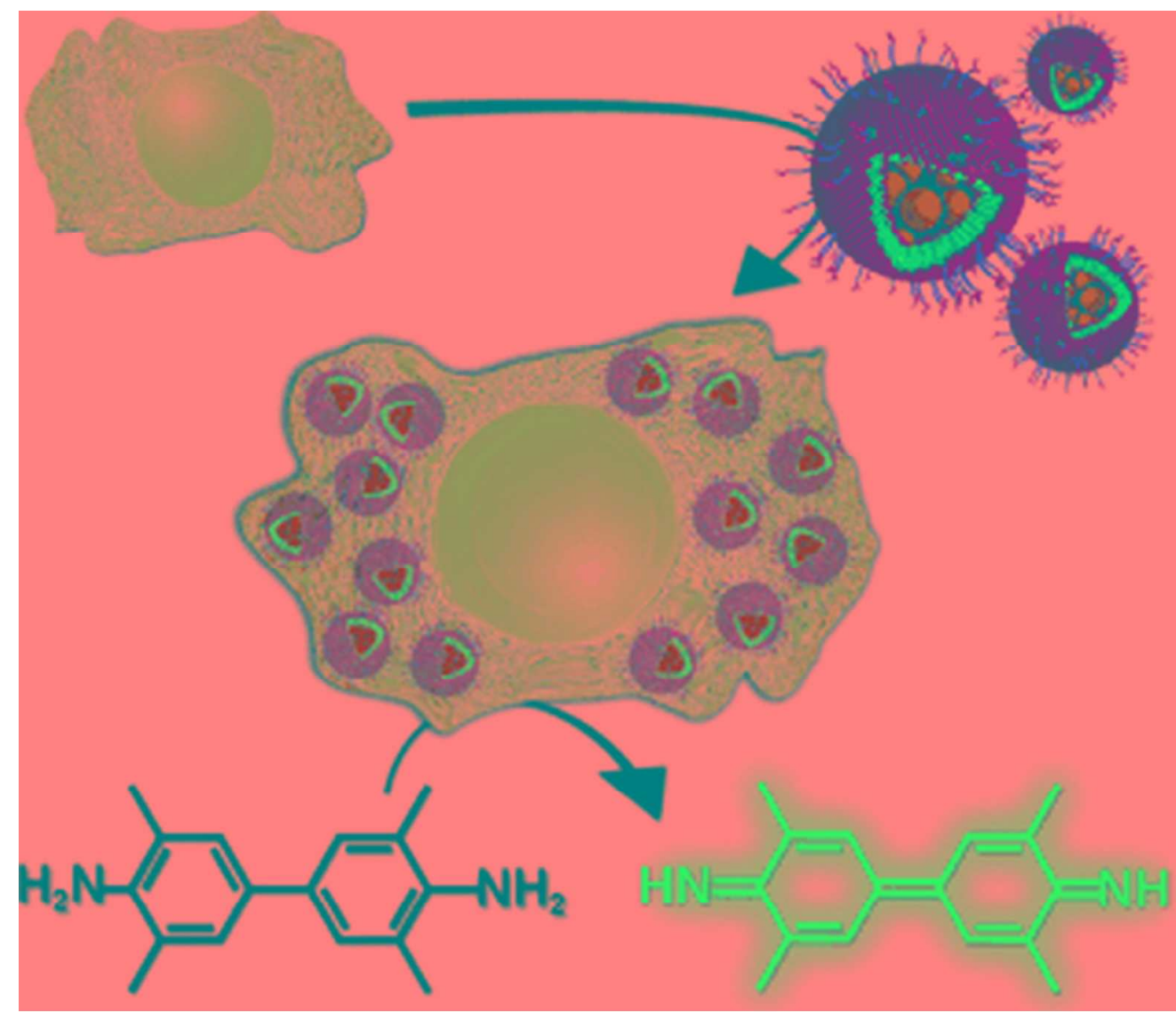

$51 \times 44 \mathrm{~mm}(300 \times 300$ DPI $)$ 
1

2

3

4

5

6

7

8

10

11

12

14

15

16

17

18

19

20

21

22

23

24

25

26

27

28

29

30

31

32

33

34

35

36

37

38

39

40

41

42

43

44

45

46

47

48

49

50

51

52

53

54

55

56

57

58

59

60

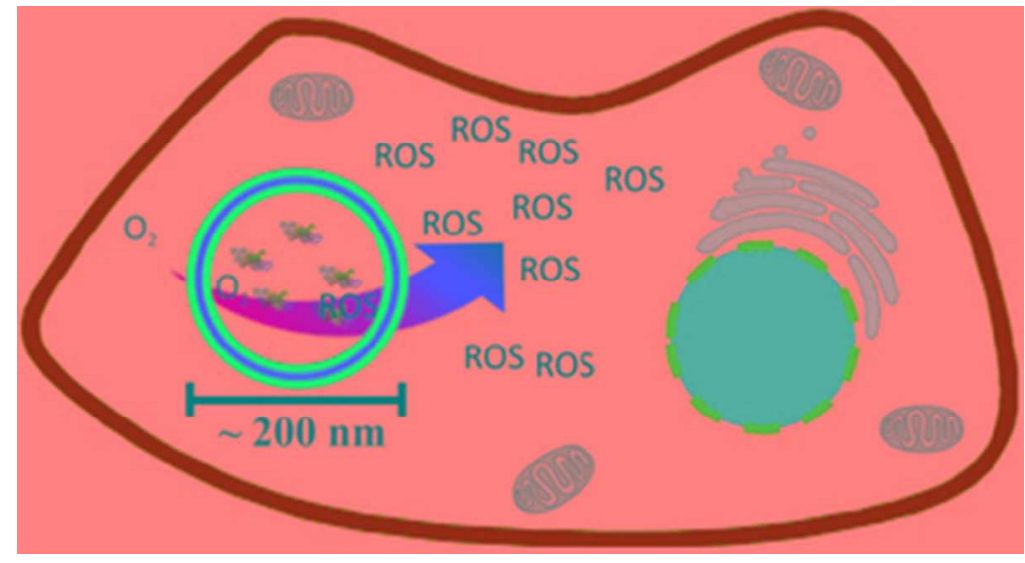

$32 \times 17 \mathrm{~mm}(300 \times 300$ DPI $)$ 


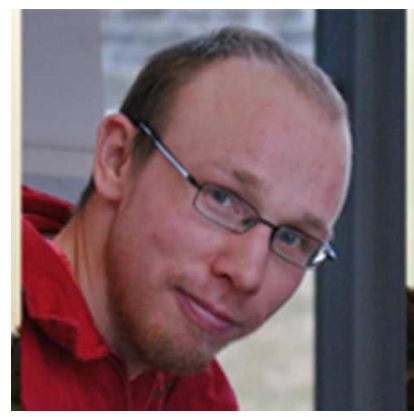

author picture Gaitzsch $54 \times 54 \mathrm{~mm}(72 \times 72$ DPI $)$ 

$34 \times 46 \mathrm{~mm}$ (300 x 300 DPI) 


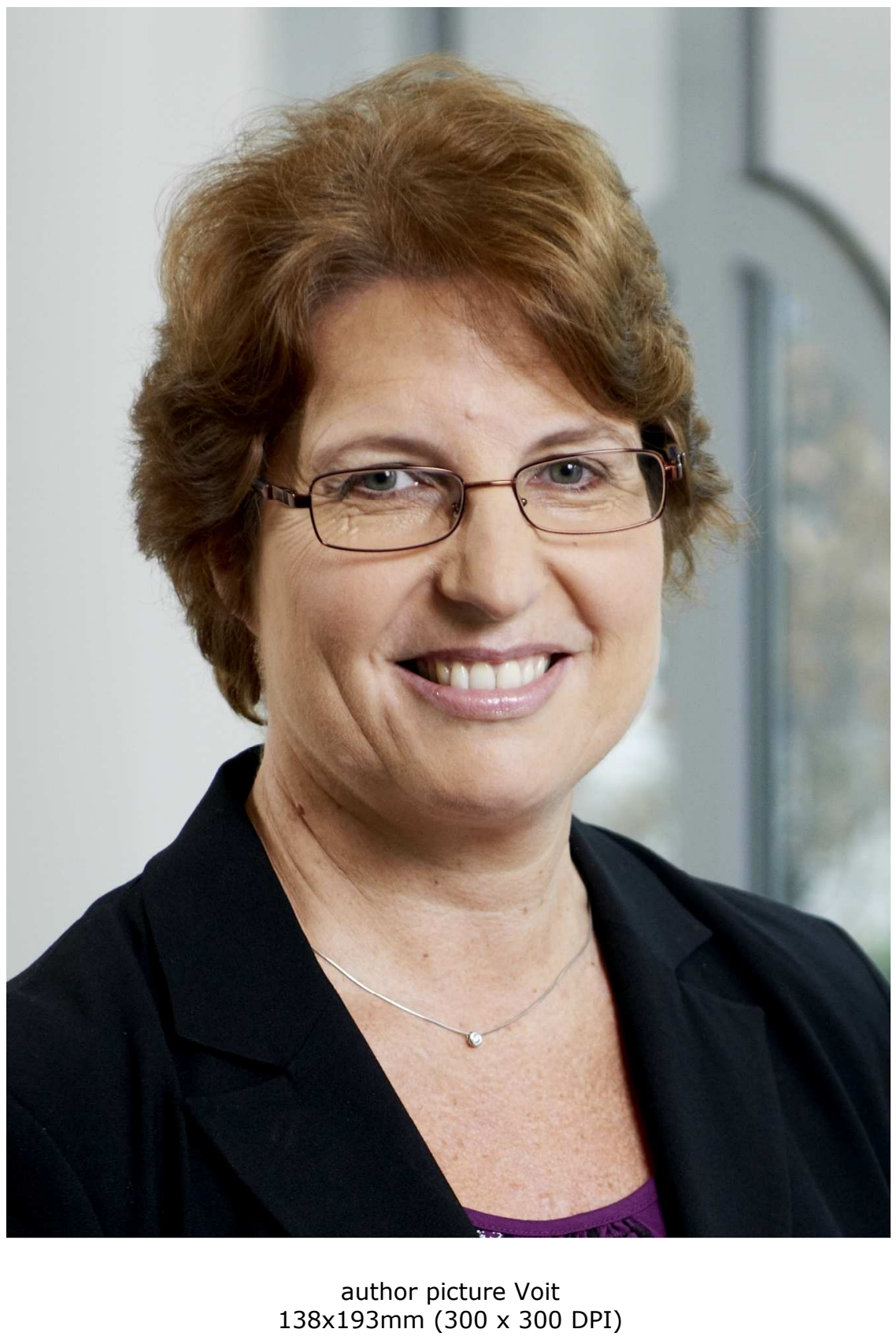

